\title{
Dynamic Aeroelastic Response Attenuation of Aerospace Structures Employing Passive Tuned Mass Dampers
}

$$
\text { by }
$$

Victor Emmanuel Liete Gasparetto

B.Eng. in Mechanical Engineering

\section{A thesis submitted to the Faculty of Graduate and Postdoctoral Affairs in partial fulfillment of the requirements for the degree of}

Master of Applied Science

in

Mechanical Engineering

Carleton University

Ottawa, Ontario

(C) 2021, Victor Emmanuel Liete Gasparetto 
"If I have seen further, it is by standing on the shoulders of Giants."

Isaac Newton, 1643 - 1727 


\begin{abstract}
This thesis proposes an optimization procedure to achieve the best configuration of Multiple Degrees of Freedom (MDOF) tuned mass dampers (TMD) to mitigate the global dynamic aeroelastic response of aerospace structures. The TMD design parameters are investigated in terms of their individual mass, stiffness, damping, and location on the target structure. In order to determine the optimum sets of TMD, a Multi-Objective design optimization employing Genetic Algorithm (MOGA) is implemented, where the selected fitness functions for the analysis are the minimization of the total mass included with the resonators and concurrent minimization of the peak displacement at a specific structural nodes in space.
\end{abstract}

Two case studies are presented where the method proposed is tested to minimize the pointing error of large Earth-based radio antenna structures. Fourteen different operational scenarios of wind gust are considered employing two models of atmospheric disturbances, namely, the Power Spectral Density (PSD) represented by the Davenport spectrum (DS), and the Tuned Discrete Gust (TDG) where the dynamic aeroelastic response of the structure is analyzed in the frequency and the time domains, respectively.

In the first case study, the inclusion of multiple-TMD in the antenna structure is analyzed and compared with different placement configurations of the multiple-TMD devices. Here, the performance of the antenna structure in the form of its pointing error and the corresponding multiple-TMD inclusion are discussed at length. It is found that the placement of the multipleTMD in the primary reflector of the antenna provides a maximum reduction in the pointing error of the antenna of $62.0 \%$ and $39.2 \%$ while subject to PSD and TDG gust disturbances , respectively. 
In the second case study, a single-TMD configuration is investigated to concurrently attenuate the aeroelastic response of all 14 operational cases considering the frequency and time-domain gust models. It is found that the optimal solutions are capable of reducing the structural response by an average of $66 \%$ and $50 \%$ for the PSD and TDG gust excitation scenarios, respectively, with a mass inclusion of $1 \%$ of the total mass of the antenna structural.

Finally, this study proposes an advanced framework to estimate the optimal parameters of TMD attenuation devices, under complex loadings conditions, as an initial step in the direction of the use of such passive systems in applications that commonly employ active or semi-active attenuation solutions.

Keywords: tuned mass dampers, structural control, multi-objective genetic algorithm optimization, dynamic aeroelastic response, Davenport spectrum, tuned discrete gust, time consistent loads and displacements, pointing accuracy 


\section{Preface}

This research project is an original work developed by Victor Gasparetto, under the supervision of Professor Mostafa El Sayed, conforming to all mandatory requirements as stated by Carleton University. The project is part of the InterTronic Solutions and NASA initiative to develop highfidelity VLBI ground stations. In close collaboration, InterTronic Solutions provided data relevant to the aeroelastic numerical model, which is used as part of the case study in chapter 6 and 7 . The following work, developed during the M.A.Sc. research, were published in peer-reviewed conferences and journals:

[1] Victor EL Gasparetto, Mostafa SA ElSayed. "Shape transformers for phononic band gaps tuning in two-dimensional Bloch-periodic lattice structures", European Journal of Mechanics A/Solids, 2021.

[2] Victor EL Gasparetto, Mostafa SA ElSayed. "Multiscale Optimization of Specific Elastic Properties and Microscopic Frequency Band-gaps of Architectured Microtruss Lattice Materials", International Journal of Mechanical Sciences, 2021.

[3] Victor EL Gasparetto, Mostafa SA ElSayed. "Shape transformers for band gaps customization of Bloch-periodic triangular lattice structures”, AIAA SciTech 2021 Forum, 2021.

At the time of writing, Chapter 6 and Chapter 7 are under preparation for submission to publication in the form of journals titled "Multi-objective Optimization of Multiple Tuned Mass Dampers for Attenuation of Dynamic Aeroelastic Response in Aerospace Structures" and "Multi-objective design optimization of passive tuned mass damper for pointing accuracy control of VLBI Earthbased radio antennas subjected to atmospheric turbulence", respectively. 


\section{Acknowledgements}

This brief piece of research is the result of countless hours of hard work, dedication, curiosity, and willingness to always deliver the best. I am sure that it would not be possible to achieve this accomplishment without the support from the kind people I met during this journey, and therefore I am pleased to thank all that were involved in the process. Firstly, to my supervisor Prof. Mostafa El Sayed, who provided me with outstanding guidance and mentoring throughout the Master's program, and also for being one of the most trustworthy people I ever had the pleasure to meet.

To all the engineering team at Intertronic Solutions that, even though in a brief encounter, contributed with the execution of this research and provided insights that were definitive to come up with technical solutions. To all Carleton University's administrative personal, specially to Neil McFadyen, Bruce Johnston, and the members of the machine shop, for allowing the effective continuation of my research, despite the occurrence of the COVID-19 pandemic.

Last but not least, I would like to thank the support of my family in Brazil, for always being by my side, and hearing me out during difficult moments I faced. And also to the family I found here in the land of maple syrup, who welcomed me in a way that could not be better! Thanks to all people from the Minto Centre 3041 office and my research group, specially to my very best friends Brendan, Chris, Leigh, and Bill. Thank you all for this astonishing experience. 


\section{Table of Contents}

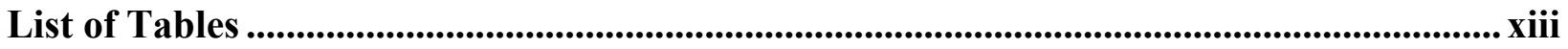

List of Figures......................................................................................................................................... xiv

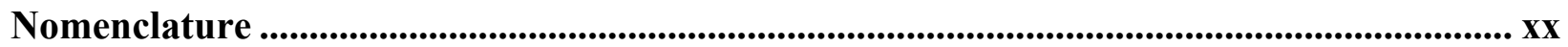

Acronyms ........................................................................................................................................ Xxvii

Chapter 1: Introduction ....................................................................................................................... 1

$1.1 \quad$ Background and motivation.....................................................................................

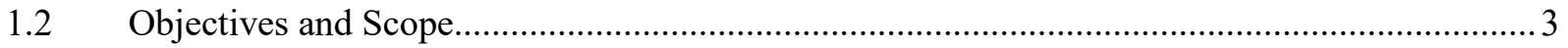

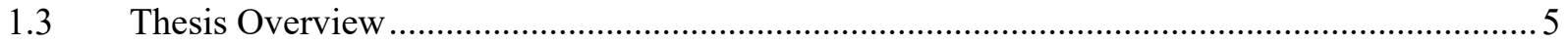

Chapter 2: Literature review ...................................................................................................... 7

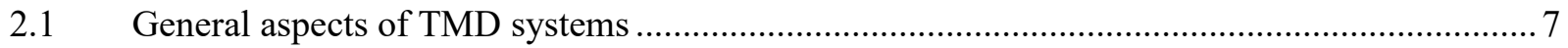

$2.2 \quad$ Passive TMD devices ......................................................................................

2.2.1 Translational TMD systems ……………………………………………………....

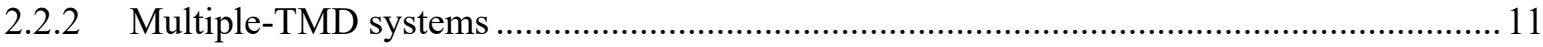

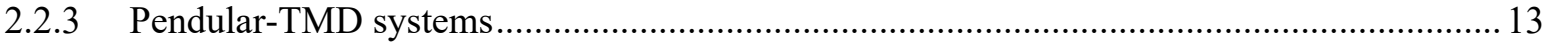

2.2.4 Tuned Liquid Column Damper systems........................................................................... 14

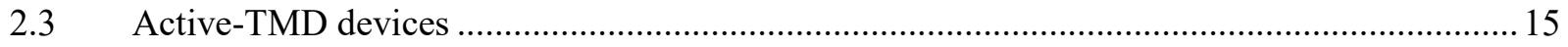

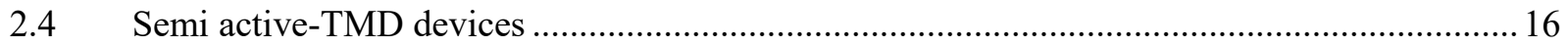




\section{Chapter 3: Theoretical framework part I - Numerical modelling of aerospace structures with multiple-tuned mass dampers ................................................................................... 19}

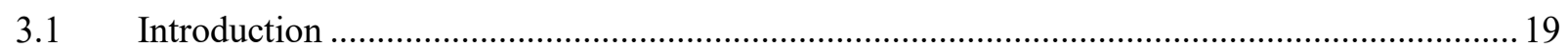

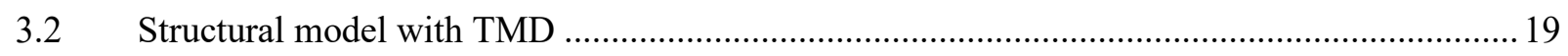

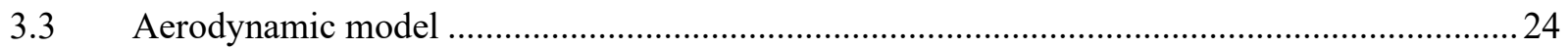

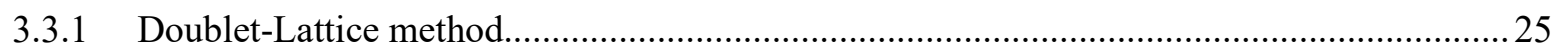

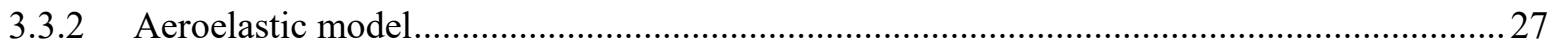

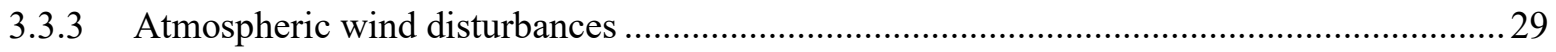

3.4 Time Consistent Loads and Displacements formulation .................................................... 31

Chapter 4: Theoretical framework part II - Optimization with genetic algorithms .......... 34

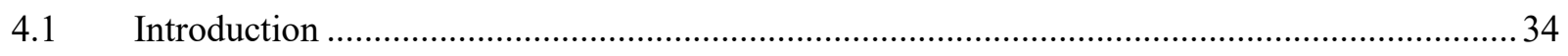

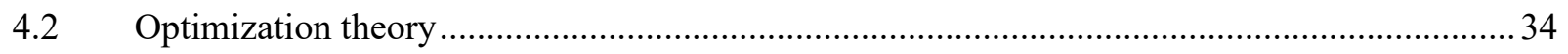

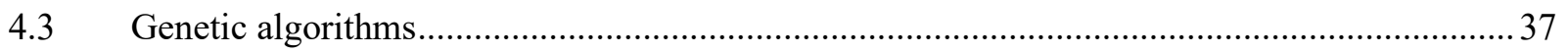

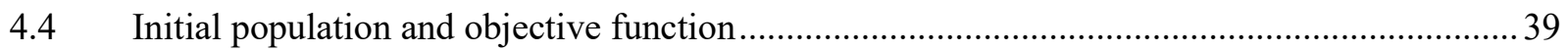

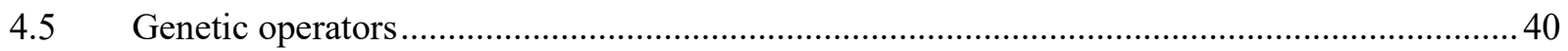

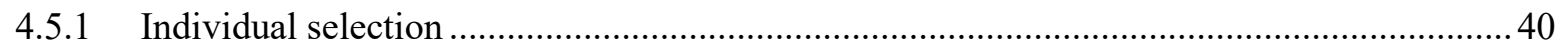

4.5.1.1 Proportional selection (Roulette - Wheel method) …......................................... 40 


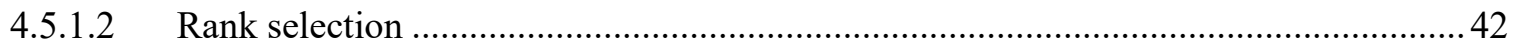

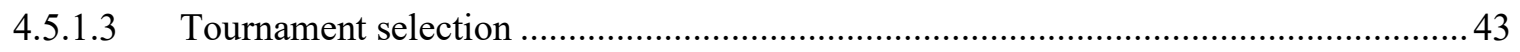

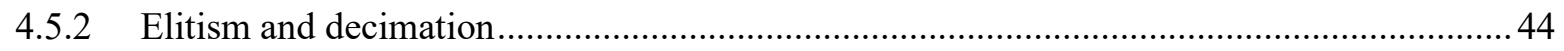

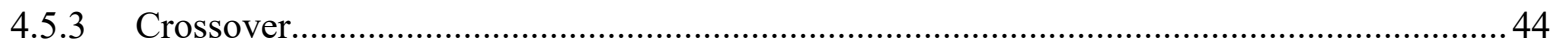

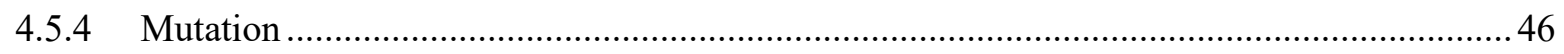

\section{Chapter 5: Theoretical framework part III - Implementation of a multi-objective design optimization procedure ............................................................................................................ 48}

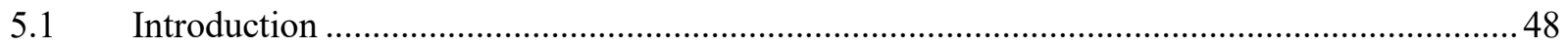

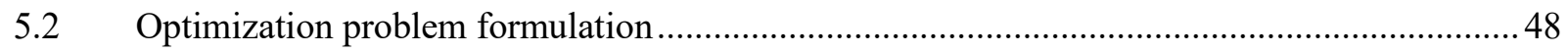

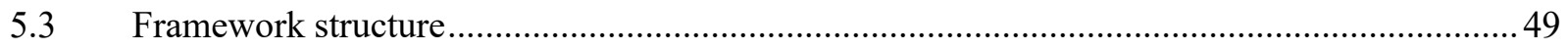

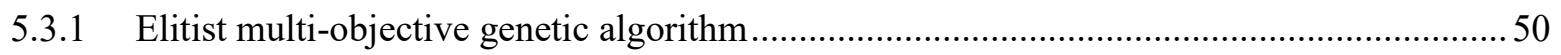

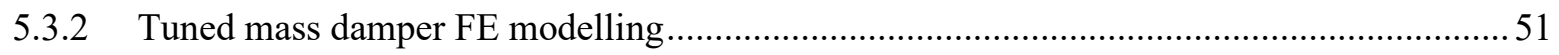

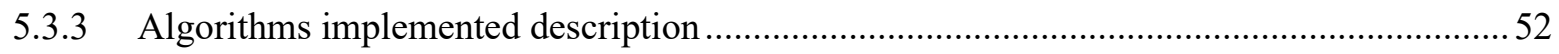

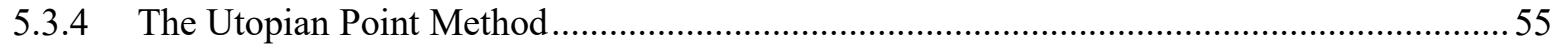

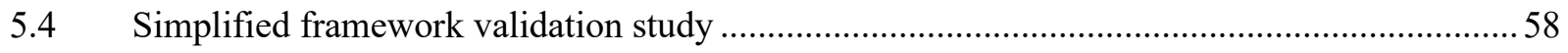

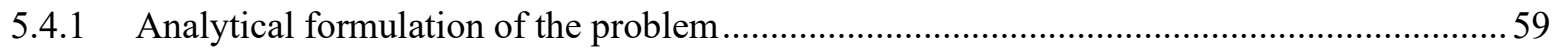

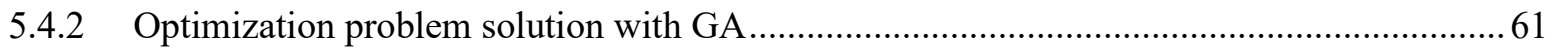




\section{Chapter 6: Case study I - Aeroelastic response attenuation of a simplified Earth-based radio}

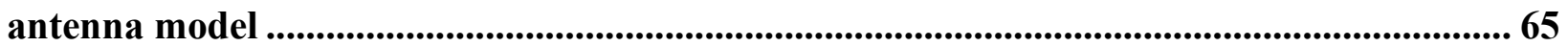

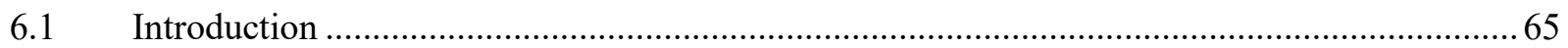

6.2 Finite element model of the Earth-based radio antenna structure ..........................................66

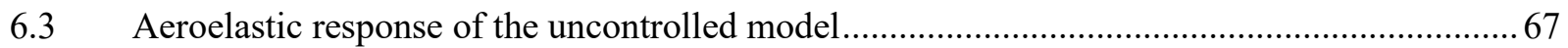

6.4 Aeroelastic response attenuation using optimal multiple-TMD ...........................................71

6.4.1 Frequency domain analysis with the PSD modelled with the Davenport Spectrum .............74

6.4.2 Time domain analysis with the One-minus cosine gust model ...................................... 78

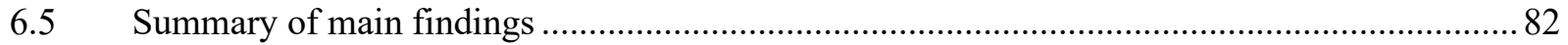

\section{Chapter 7: Case study II - Pointing error reduction of a 12m Earth-based radio VLBI

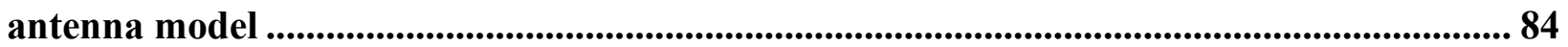

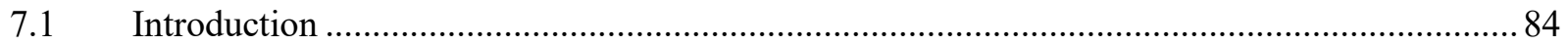

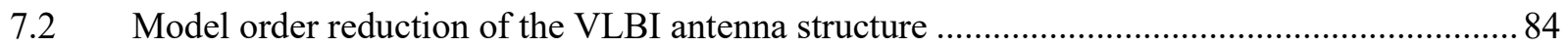

7.2.1 Superelement implementation and ROM consistency verification .................................. 85

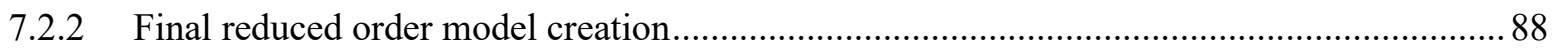

7.3 Uncontrolled VLBI antenna structure aeroelastic response …............................................ 92

7.3.1 Operational cases gust profiles.............................................................................. 93

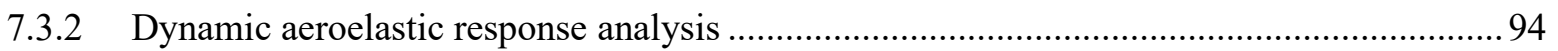

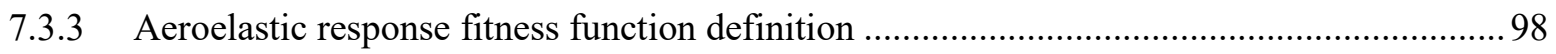


7.4 Response attenuation using optimized single-TMD. 100

7.4.1 Frequency domain analysis with the PSD modelled with the Davenport Spectrum. 103

7.4.2 Time domain analysis with the One-minus cosine gust model. 108

7.4.3 Comparison of the aeroelastic response fitness for the gust models

7.4.4 Factorial analysis of TMD design variables with a defined mass inclusion

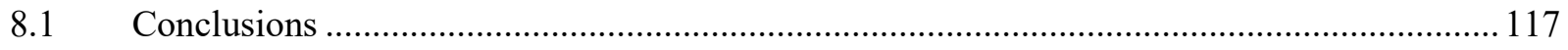

8.2 Recommendations for Future Work ....................................................................... 120

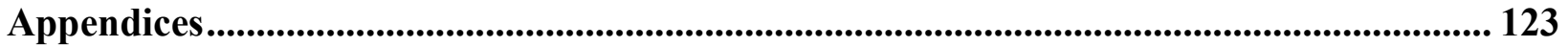

Appendix A - MATLAB ${ }^{\circledR}$ routines for the framework validation................................................... 123

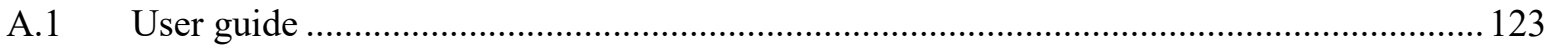

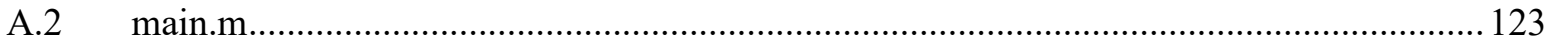

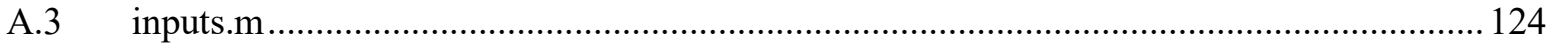

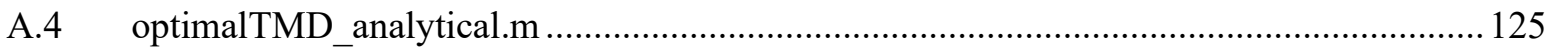

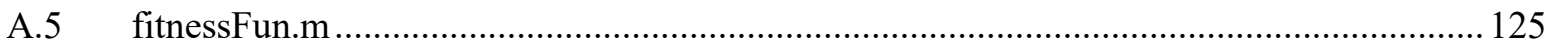

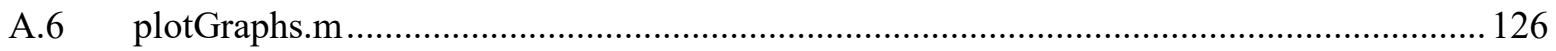

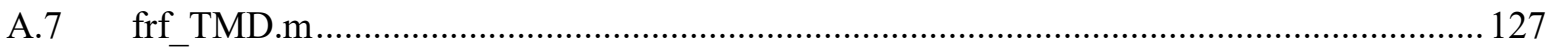

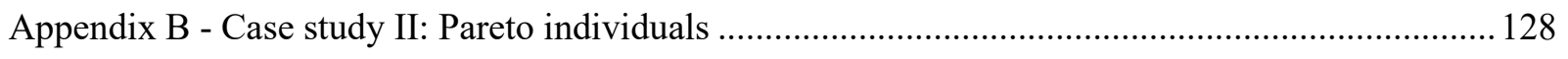




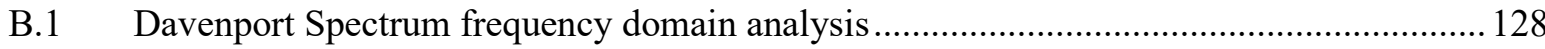

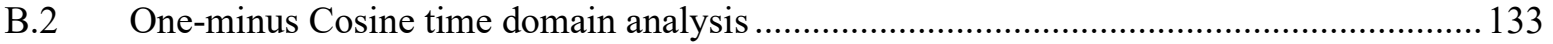

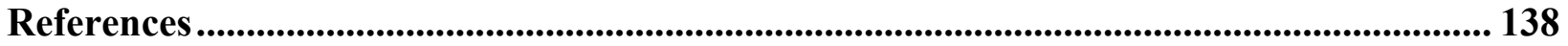




\section{List of Tables}

Table 4.1: Common nomenclature utilized in genetic algorithms context

Table 5.1: Selection of optimal design variables comparing the analytical and GA solutions

Table 6.1: Operational cases summary [38]

Table 6.2: Evaluation of the fitness function for the uncontrolled antenna structure

Table 6.3: Multiple-TMD identification according to the grid point numbering of the FE model of the Earth-based antenna

Table 6.4: Design variables bounds for each configuration investigated

Table 6.5: Pareto front values for the best individuals under the DS gust excitation model

Table 6.6: Pareto front values for the best individuals under the TDG gust excitation model

Table 6.7: Summary of the best values for the multiple-TMD masses for the Configuration 1 under the operational scenarios modelled with the DS and TDG

Table 7.2: Operational cases for the VLBI antenna structure [38]

Table 7.3: Frequencies comparison between DFEM and the new ROM developed

Table 7.4: Design variables bounds for the single-TMD analysis

Table 7.5: Best TMD individuals for the PSD and TDG excitation models 


\section{List of Figures}

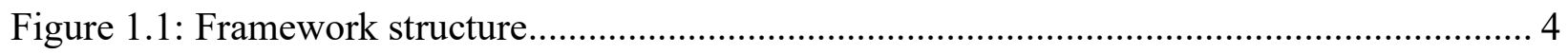

Figure 3.1: FE model of a generic structure with the inclusion of multiple-TMD 20

Figure 4.1: Minimization of a function $f$, where $\boldsymbol{x} \mathbf{1}$ is the global minimizer variable, and $\boldsymbol{f}$ is the optimal value... 36

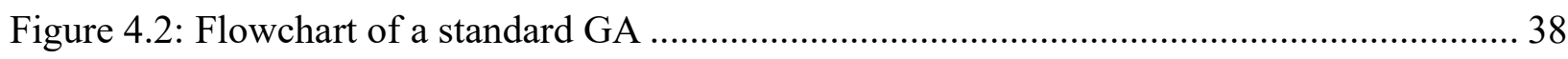

Figure 4.3: Components of a binary chromosome. 39

Figure 4.4: Roulette-Wheel selection method diagram with the probability of selection of each individual 41

Figure 4.5: Tournament selection schematic. 43

Figure 4.6: Single point crossover with binary codification 45

Figure 4.7: (a) Two point random and (b) Fully random crossover. The arrows indicate the respective allele that will go through the crossover operation 46

Figure 4.8: Binary codification mutation operation. 47

Figure 5.1: Schematics of the FE model of a translational TMD. 51

Figure 5.2: MSC NASTRAN ${ }^{\circledR}$ cards specification for the creation of TMD 52

Figure 5.3: Flowchart of the optimization routine developed. 53 
Figure 5.4: Utopian point of a Pareto front. The points A and B are the extremity individuals of the Pareto front, $\mathrm{C}$ represents a point identified by the utopian metric utilized 56

Figure 5.5: Undamped SDOF system coupled with a damped TMD device. 59

Figure 5.6: Results for the validation case study. (a) Amplitude of response H1 with respect to the excitation frequency ratio for the solutions obtained with the analytical model and the GA framework. (b) Comparison of the optimal individuals obtained with the GA. 63

Figure 6.1: Schematics visualization of the FE model of the Earth-based radio antenna structure investigated 67

Figure 6.2: DLM panels modelling of the aerodynamic surfaces of the Earth-based antenna frame 68

Figure 6.3: TDG and PSD function of the DS considered in the study for 14 operational conditions. The velocities indicated in the color bar are the mean wind speed for the operational scenarios for both gust models

Figure 6.4: Aeroelastic response of the target node of the uncontrolled antenna structure under the operational cases. PSD wind turbulence for the (a) primary and (b) secondary scenarios. TDG for the (c) primary and (d) secondary scenarios 71

Figure 6.5: Node numbering description for the Earth-based antenna FE model 72

Figure 6.6: Pointing error minimization using the multiple-TMD under the configuration 1 for the primary operational case modelled with the PSD function of the DS. (a) Multi-objective Pareto front of all individuals analyzed, indicating the utopian point and the best individual. (b) Frequency 
response functions of the deviation angles for each respective direction, where the color bar indicated represents the percentage of mass inclusion in the antenna..... 75

Figure 6.7: Comparison of Pareto fronts between all configurations investigated in the study for both operational scenarios modelled with the DS. (a) Primary and (b) Secondary cases 77

Figure 6.8: Comparison of the frequency response functions, for the $\boldsymbol{\Theta} \mathrm{DOF}$, considering all best individuals of each configuration investigated, obtained with the utopian point method. The (a) graph presents the results for the primary operational case modelled with the DS, while the (b) graph represents the secondary operational case ....... 77

Figure 6.9: Pointing error minimization using the multiple-TMD under the configuration 1 for the secondary operational case modelled with the TDG. (a) Multi-objective Pareto front of all individuals analyzed, indicating the utopian point and the best individual. (b) Time response functions of the RSSQ deviation angle for each respective direction, where the color bar indicated represents the percentage of mass inclusion in the antenna 79

Figure 6.10: Comparison of Pareto fronts between all configurations investigated in the study for both operational scenarios modelled with the TDG. (a) Primary and (b) Secondary cases 80

Figure 6.11: Comparison of the time response functions, for the RSSQ deviation angle, considering all best individuals of each configuration investigated, obtained with the utopian point method. The (a) graph presents the results for the primary operational case modelled with the TDG, while the (b) graph represents the secondary operational case. 81

Figure 6.12: Summary of the multiple-TMD Configuration 1 optimal damping and stiffness values, considering both operational cases modelled with the (a-b) DS and the (c-d) TDG 83 
Figure 7.1: External superelement creation and verification of ROM consistency..... 87

Figure 7.2: InterTronic Solutions 12m VLBI antenna FE model evolution 89

Figure 7.3: Percentage error evaluated for the natural frequencies of the first 25 flexible modes for the ROM - Gasparetto (2021) and Nieto et al. (2020) 90

Figure 7.4: First 25 normal modes correlation between the ROM - Gasparetto (2021) and Nieto et al. (2020) 91

Figure 7.5: Reference and target monitoring grid points chosen for the VLBI antenna 92

Figure 7.6: One-minus cosine and Davenport Spectrum gust models for the 14 operational cases analyzed 94

Figure 7.7: Uncontrolled structure response utilizing the PSD excitation modelled with DS. (a) Target node PSD displacement functions of the operational scenarios considered. (b) Correspondent mode shapes for the first two resonance frequencies 95

Figure 7.8: Target node time-response displacement of the operational scenarios considered for the InterTronic VLBI antenna, utilizing the TDG model 96

Figure 7.9: Fitness function evaluated for the uncontrolled structure under the (a) PSD excitation modelled with the DS and (b) One-minus cosine gust models..... 100

Figure 7.10: Multi-objective Pareto front of all individuals analyzed, indicating the utopian point and the best individual using the single-TMD for the operational cases 4, 6, 8 and 10 modelled with the DS model 104 
Figure 7.11: Concurrent aeroelastic response minimization using the single-TMD configuration for the operational cases 4, 6, 8 and 10 modelled with the DS. The PSD displacement functions of the target node are presented for each respective direction, where the color bar indicated represents the percentage of mass inclusion in the antenna 106

Figure 7.12: Aeroelastic response fitness function comparison between the uncontrolled and the controlled structure with an optimal single-TMD. Results presented for the Davenport Spectrum model. 107

Figure 7.13: Multi-objective Pareto front of all individuals analyzed, indicating the utopian point and the best individual using the single-TMD for the operational cases $4,6,8$ and 10 modelled with the One-minus cosine gust model

Figure 7.14: Concurrent aeroelastic response minimization using the single-TMD for the operational cases under the One-minus cosine gust model. Time response functions of the target node displacement are presented as a RSSQ of each DOF, where the color bar indicated represents the percentage of mass inclusion in the antenna....... 110

Figure 7.15: Aeroelastic response fitness function comparison between the uncontrolled and the controlled structure with an optimal single-TMD. Results presented for the One-minus cosine gust model. 111

Figure 7.16: Comparison of the aeroelastic response fitness for the (a) PSD and (b) TDG gust models. The best individuals found from the optimization framework are evaluated among both gust models 114 
Figure 7.17: Factorial analysis of design variables considering a constant mass inclusion, for the operational case 6. (a) Davenport Spectrum and (b) One-minus cosine gust models 


\section{Nomenclature}

Latin Characters

\begin{tabular}{ll}
\hline Symbol & Definition \\
\hline $\mathbf{A}_{j j}$ & Aerodynamic Influence Coefficient matrix \\
$b$ & Airfoil semi-chord \\
$\mathbf{C}$ & Damping matrix of the structure \\
$C$ & Number of Pareto front individuals \\
$\tilde{\mathbf{C}}$ & Damping matrix modified by the inclusion of TMD \\
$\tilde{\mathbf{C}}_{q}$ & Normalized damping matrix modified by the inclusion of TMD \\
$c_{q_{j}}$ & j-th modal damping coefficient \\
$c_{d}$ & TMD viscous damping coefficient \\
$\mathbf{c}_{T M D}$ & Vector with each damping of the TMD devices \\
$d$ & Number set range contained in the $\mathrm{R}^{\mathrm{n}}$ \\
$D$ & Minimum utopian metric \\
$\mathbf{D}_{j k}^{1}$ & Substantial differentiation matrix 1 \\
$\mathbf{D}_{j k}^{2}$ & Substantial differentiation matrix 2 \\
error & Percentage error assessed between two measured values \\
$f$ & Ratio of natural frequency of the TMD and the structure \\
$\tilde{f}$ & Optimal value of a function \\
$f(i)$ & Column vector of non-aerodynamic forces \\
$f(x)$ & Fitness value \\
$\mathbf{\mathbf { F }}(t)$ & General function dependent on the x variable \\
\hline &
\end{tabular}




\begin{tabular}{|c|c|}
\hline $\mathbf{F}^{0}$ & Utopian point \\
\hline $\boldsymbol{f}_{j}$ & Normalized lifting pressure at the $\mathrm{j}$-th point \\
\hline $\mathbf{G}_{k d}$ & Structural splining matrix \\
\hline$H$ & Amplification factors associated with the response \\
\hline $\mathbf{H}(\omega)$ & Transfer function \\
\hline $\mathbf{K}$ & Stiffness matrix of the structure \\
\hline$k$ & Dimensionless reduced frequency \\
\hline$\widetilde{\mathbf{K}}$ & Stiffness matrix modified by the inclusion of TMD \\
\hline$\widetilde{\mathbf{K}}_{q}$ & Normalized stiffness matrix modified by the inclusion of TMD \\
\hline$k_{q_{j}}$ & $\mathrm{j}$-th modal stiffness coefficient \\
\hline$k_{d}$ & TMD stiffness \\
\hline$k_{S}$ & Structure stiffness \\
\hline $\mathbf{k}_{T M D}$ & Vector with each stiffness of the TMD devices \\
\hline$L_{g}$ & Gust length \\
\hline $\mathbf{M}$ & Diagonal mass matrix of the structure \\
\hline m & Individual mass inclusion of TMD \\
\hline$\widetilde{\mathbf{M}}$ & Mass matrix modified by the inclusion of TMD \\
\hline $\bar{m}$ & Mass ratio between the TMD and the structure \\
\hline$\widetilde{\mathbf{M}}_{q}$ & Normalized mass matrix modified by the inclusion of TMD \\
\hline$m_{q_{j}}$ & j-th modal mass \\
\hline$m_{d}$ & TMD mass \\
\hline$m_{s}$ & Structure mass \\
\hline $\mathbf{m}_{T M D}$ & Vector with each mass of the TMD devices \\
\hline$N$ & Population size \\
\hline
\end{tabular}




$\begin{array}{ll}N_{c a s e} & \text { Number of operational cases } \\ N_{D O F} & \text { Number of degrees of freedom } \\ N_{D O F} & \text { Number of DOF considered } \\ n_{T M D} & \text { Number of TMD devices } \\ p & \text { Tournament size parameter } \\ \hat{p} & \text { Amplitude of displacement of the harmonic excitation } \\ \widetilde{\mathbf{P}}(t) & \text { External forces column vector considering TMD degrees of freedom } \\ \widetilde{\mathbf{P}}(\omega) & \text { Modal forces vector in the frequency domain } \\ \mathbf{P}(t) & \text { External forces column vector in structural nodes } \\ P_{c} & \text { Crossover probability } \\ P_{i} & \text { i-th probability } \\ \mathbf{P}_{k} & \text { Aerodynamic forces } \\ P_{m} & \text { Mutation probability } \\ P o p & \text { Population individuals array } \\ p_{s} & \text { Sinusoidal harmonic excitation } \\ q & \text { Mandomly generated probability } \\ \mathbf{q}(\omega) & \text { Metric order for the utopian point method } \\ \mathbf{Q}_{d d} & \text { Harmonic modal response in the frequency domain } \\ q_{i} & \text { Matrix of Force Aerodynamic Influence coefficients } \\ \mathbf{q}_{i}(t) & \text { coordinates } \\ \mathbf{Q}_{k k} & \mathbf{Q}_{q}\end{array}$




\begin{tabular}{|c|c|}
\hline$S_{D}(\omega)$ & Davenport Spectrum PSD function \\
\hline $\mathbf{S}_{k j}$ & Integration matrix \\
\hline$t$ & Time \\
\hline$t_{f}$ & Final time analyzed \\
\hline$U$ & Time consistent variable (e.g. load, displacement, etc.) \\
\hline$\hat{u}$ & Amplitude of displacement of the structure \\
\hline$\hat{u}_{d}$ & Amplitude of displacement of the TMD \\
\hline $\mathbf{u}(t)$ & Nodal displacements column vector \\
\hline$u_{d}$ & TMD degree of freedom displacement \\
\hline$U_{\text {fitness }}$ & Fitness function related to the aeroelastic response \\
\hline $\mathbf{u}_{k}$ & Aerodynamic displacements \\
\hline$U_{R S S Q}$ & Root sum of squares of degrees of freedom displacement \\
\hline$u_{s}$ & Structure degree of freedom displacement \\
\hline$v$ & Wind gust velocity \\
\hline$V_{\infty}$ & Free stream velocity \\
\hline$v_{g 0}$ & Peak gust disturbance or design gust velocity \\
\hline$w_{i}$ & i-th weight for the utopian point method \\
\hline $\boldsymbol{w}_{j}$ & Downwash vector \\
\hline $\mathbf{x}(t)$ & $\mathrm{x}$-displacement degrees of freedom of the structure's nodes \\
\hline$x_{g}$ & Position in the spatial coordinates of the gust with respect to a fixed origin \\
\hline $\mathbf{y}(t)$ & $y$-displacement degrees of freedom of the structure's nodes \\
\hline$z$ & Height from the ground up to the center of the structure \\
\hline$z_{0}$ & Height of the terrain roughness \\
\hline$\Delta L$ & Fixed offset distance between the target and reference nodes \\
\hline
\end{tabular}




\begin{tabular}{ll}
\hline Symbol & Definition \\
\hline $\boldsymbol{\Phi}_{R O M-i}$ & Mode shape of the $i$-th ROM \\
$\xi_{d}$ & Damping ratio of the TMD device \\
$\rho_{\text {air }}$ & Air density \\
$\omega_{n_{i}}$ & $i$-th natural frequency \\
$\omega_{R O M-i}$ & Natural frequencies of the $i$-th ROM \\
$\omega_{d}$ & TMD circular frequency \\
$\boldsymbol{\varphi}_{i}$ & $i$-th mode shape \\
$\xi$ & Vector of design variables from the optimization problem \\
$\Theta$ & Deviation angle associated with the $x$-direction \\
$\Phi$ & Deviation angle associated with the $y$-direction \\
$\Psi$ & Deviation angle associated with the $z$-direction \\
$\Omega$ & Excitation frequency \\
$\alpha$ & Percentage value for mass inclusion \\
$\delta$ & Phase angle between response and excitation \\
$\kappa$ & Surface drag coefficient \\
$\rho$ & Ratio of the excitation frequency and the natural frequency of the structure \\
\hline & Phase angle \\
\hline & Circular frequency \\
\hline &
\end{tabular}




\begin{tabular}{ll}
\hline Symbol & Definition \\
\hline$\left(^{\cdot}\right)$ & First derivative with respect to time \\
$\left({ }^{*}\right)$ & Second derivative with respect to time \\
()$^{T}$ & Transpose operator \\
$\infty$ & Infinity \\
$\Delta(\quad)$ & Variation \\
$\min (\quad)$ & Minimization operator \\
$\max (\quad)$ & Maximization operator \\
\hline
\end{tabular}

Subscripts

\begin{tabular}{ll}
\hline Symbol & Definition \\
\hline aero & Aerodynamic \\
crit & Critical \\
gen & Generalized coordinates \\
max & Maximum \\
m & Mean value \\
min & Minimum \\
opt & Optimal \\
stn & Station \\
$T C L D$ & Time Consistent Loads and Displacements quantity \\
$T M D$ & Tuned mass damper attached
\end{tabular}




$\begin{array}{ll}U N C & \text { Uncontrolled } \\ x & x \text {-direction } \\ y & y \text {-direction } \\ z & z \text {-direction }\end{array}$




\section{Acronyms}

\begin{tabular}{ll}
\hline Acronym & Definition \\
\hline AIC & Aerodynamic Influence Coefficients \\
CBRM & Craig-Bampton Reduction Method \\
CPU & Central Processing Unit \\
DB-set & Database set \\
DFEM & Detailed Finite Element Model \\
DLM & Doublet-Lattice Method \\
DMAP & Direct Matrix Abstraction Program \\
DOF & Degree of Freedom \\
DS & Davenport Spectrum \\
EOM & Equation of Motion \\
FE & Finite Element \\
FRF & Frequency Response Function \\
GA & Genetic Algorithm \\
ID & Identification \\
MAC & Modal Assurance Criteria \\
MDOF & Multiple Degrees of Freedom \\
MOGA & Multi-objective Genetic Algorithm \\
NASA & National Aeronautics and Space Administration \\
OTM & Output Transformation Matrices \\
PCH & Potational Inertia Double TMD \\
PSD & RIDTMD
\end{tabular}




$\begin{array}{ll}\text { ROM } & \text { Reduced Order Model } \\ \text { RSSQ } & \text { Root Sum of Squares } \\ \text { SDOF } & \text { Single Degree of Freedom } \\ \text { SE } & \text { Super Element } \\ \text { SPC } & \text { Single Point Constraint } \\ \text { SOL } & \text { Solution } \\ \text { TCLD } & \text { Time Consistent Loads and Displacements } \\ \text { TDG } & \text { Tuned Discrete Gust } \\ \text { TLCD } & \text { Tuned Liquid Column Damper } \\ \text { TMD } & \text { Tuned Mass Damper } \\ \text { VLBI } & \text { Very Long Baseline Interferometry }\end{array}$




\section{Chapter 1: Introduction}

\subsection{Background and motivation}

Aerospace structures and related equipment are subjected to a wide range of dynamic loadings. When excited close to resonance frequencies, the dynamic loads can cause excessive vibrations in the structures, resulting in loss of accuracy $[1,2]$, structural instabilities $[3,4]$ material fatigue $[5,6]$, among others, causing potential catastrophic failures. Additionally, it is always found that high levels of vibration responses normally occur in aerospace structures when subjected to aerodynamic loadings due to their inherent slenderness and lightweight design considerations $[7,8]$. On the other hand, in the last few decades, there was a significant increase in the stringent design and certification requirements for aerospace structures, such that more efficient design solutions related to material-to-strength ratio and vibration control systems are implemented aiming to improve the operation quality of those structures [9-12].

In this context, one of the major goals in the design of aerospace structures is to increase the efficiency of their dynamic responses to enhance their fatigue characteristics as well as their fluidstructure interaction stabilities. A wide range of vibration control devices have been proposed in the literature, characterized by passive, adaptive, semi-active $[13,14]$ or active nature $[15,16]$, and can be effectively implemented to achieve those design requirements. Of particular interest are the passive vibration attenuation devices. Such devices are based on the principle that a non-active system is integrated into a structure, to transfer or absorb part of the kinetic energy of global mode shapes. A particular type of passive vibration attenuation device is the Tuned Mass Damper (TMD), that essentially consists of an auxiliary mass attached to the main structure, which is tuned to partially absorb its kinetic energy. TMD's can be classified into four categories, namely, the 
tuned liquid column dampers [17], pendular-TMD [18], bidirectional and homogeneous TMD [19], and conventional TMD [20]. A good example of the effectiveness of vibration control of a conventional translational TMD in aerodynamic structures, specifically applied for racing cars, was the Renault R26 prototype, during the 2006 Formula One season [21]. Due to geometrical constraints in that year's competition, the cars were suffering from a decrease in nose pitch motion stabilization under gust excitations or when hitting curbs, which resulted in excessive vibrations in the front wing, thus reducing the aerodynamic performance and grip level of the vehicles. The inclusion of the TMD by the Renault team allowed these excessive vibrations to be damped out, thus making the racing car more stable.

Due to the ease in their implementation and robust design methodologies, TMD's have been extensively used in civil structures, such as high-rise buildings [22-25], telecommunication towers [26], wind turbines $[27,28]$ or bridges $[29,30]$ in order to mitigate seismic, hydrodynamic and aerodynamic vibrations. TMD's are also employed in aircraft wing structures for aeroelastic stability and vibration attenuations and reductions [31]. Recently, Lee et al. [32] designed a conventional TMD system to mitigate the elastic response of a large retractable truss-like frame under seismic loads excitations, achieving 90\% reduction in the vibration levels with the inclusion of 8 TMD when compared to the uncontrolled structure. Liu et al. [33] used a single-degree-offreedom (SDOF) TMD to mitigate the vibration response of fore-aft of wind turbine towers, comparing the analytical formulation and a radial basis function neural network to achieve the optimum solution.

Moreover, Ma et al. [34] investigated the efficiency of MDOF TMD applied in beam structures, showing that with such configuration is possible to attenuate multiple modes of interest while requiring less mounting space than conventional SDOF TMD systems. Additionally, the use of 
MDOF multiple-TMD devices is an approach that overcomes the limitations inherent in the use of a single-DOF TMD. This is due to the fact that the auxiliary masses are distributed throughout the target structure at designated locations, therefore permitting controlling capabilities of upper vibration modes in complex shape structures $[35,36]$.

The performance of MDOF TMD control devices depends intrinsically on the design parameters of mass, stiffness, damping, the number and location of the auxiliary masses, which define the frequency range of operation of the dynamic resonator [37]. In the case of aerospace structures, another critical factor that needs to be considered by the designer is the minimization of the total mass inclusion when using the TMD. In this way, it is pertinent to develop an efficient optimization framework for determining optimum design parameters of TMD applied to aerospace structures, under the consideration of realistic aerodynamic load scenarios, such that the performance of the control devices can be effectively quantified.

\subsection{Objectives and Scope}

The main objectives of the thesis are as follows:

- Develop a numerical framework to properly tune passive TMD devices in order to attenuate the dynamic response of aerospace structures

- Investigate the optimal parameters of the passive TMD for the case study of a Very Long Baseline Interferometry (VLBI) antenna structure model that is subjected to different forms of gust excitation, with the intent to minimize the aeroelastic response while minimizing the mass inclusion of the control devices. 
The scope of the thesis will be limited to the investigation of the structural dynamic aeroelastic performance with the integrated MDOF translational TMD and an in-depth discussion about the framework developed herein.

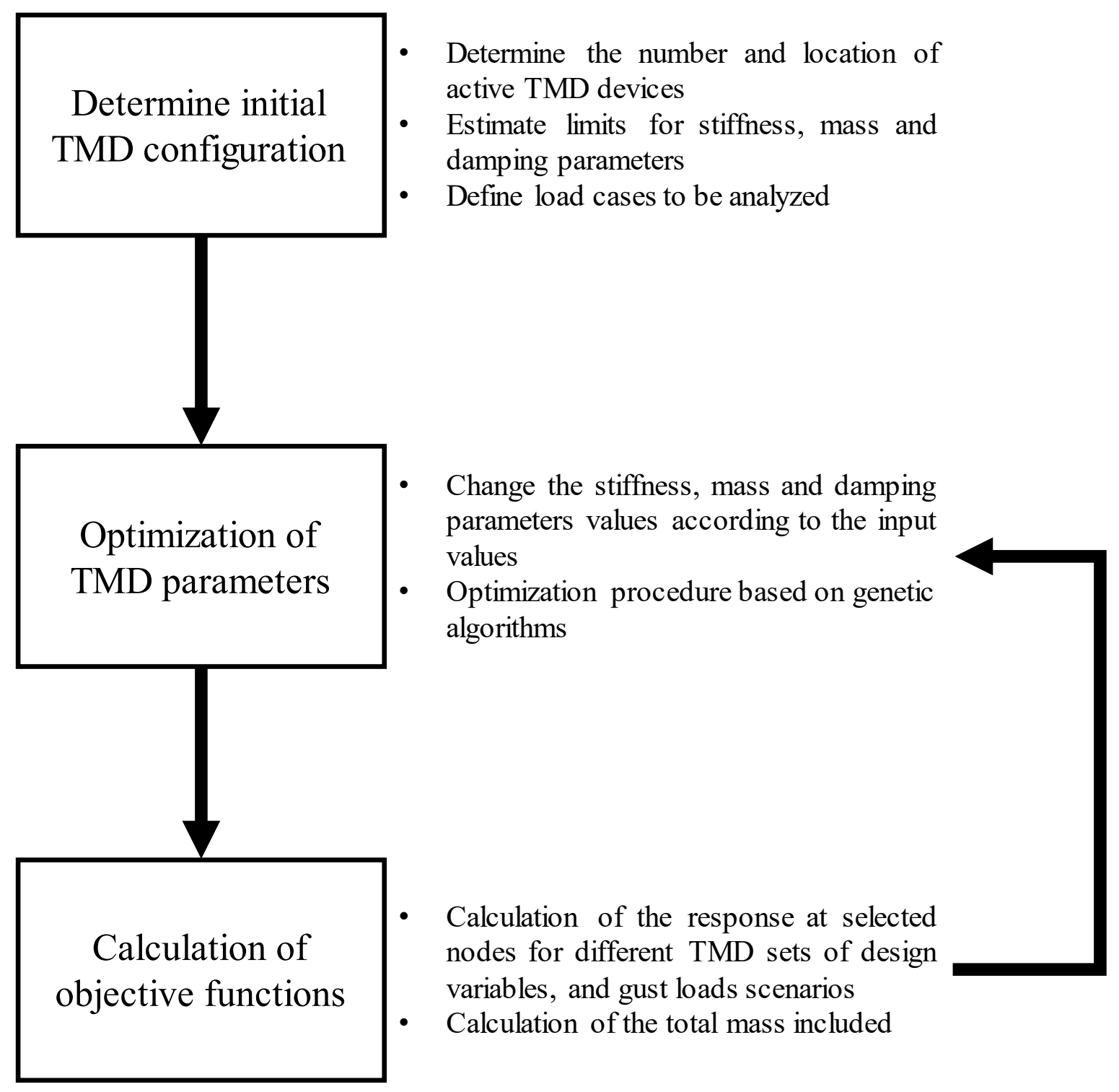

Figure 1.1: Framework structure

A simplified structure of the framework implemented is depicted in Figure 1.1. For the TMD system, the constitutive parameters considered are essentially the mass, spring stiffness and 
damping coefficient. Those are taken as design variables for the optimization procedure, and can be tuned accordingly for the dynamic aeroelastic response attenuation. Initially, the framework developed is tested with a simplified Finite Element Model (FEM) of an antenna structure, being composed of truss-like members, which is excited by two atmospheric disturbances, namely, Power Spectrum Density (PSD) gust represented by the Davenport Spectrum and the One-minus cosine TDG gust. The optimal parameters of different sets of TMD are found and discussed at length. Moreover, the framework is applied to a model of a $12 \mathrm{~m}$ VLBI antenna, designed by InterTronic Solutions [38], where the aeroelastic response for multiple operational scenarios are concurrently minimized by means of the inclusion of a passive TMD's.

\subsection{Thesis Overview}

The thesis is organized as follows:

After the Introduction, Chapter 2 presents a literature review of TMD classification, constitutive equations, and effective implementation. Major focus will be given to passive, semi-active and active TMD designs.

Chapter 3 contains the analytical formulation of the Part I of the framework developed. In the text, it is presented the formulation of the TMD in the global equation of motion (EOM) of the structural FEM. In sequence, the aeroelastic EOM is derived with the modified FE constitutive matrices, considering the excitation gust models given by a PSD modelled with the DS and the TDG. Then, the general formulation of the Time Consistent Loads and Displacement (TCLD) is proposed.

Chapter 4 provides a brief mathematical discussion about solving optimization problems using Genetic Algorithm (GA), being the Part II of the framework developed in this work. The common 
terminology utilized within this optimization method is presented, their concepts and common values utilized are discussed.

Chapter 5 presents the Part III of the theoretical framework, where the multi-objective optimization procedure to minimize the aeroelastic response of aerospace structures is introduced. The implementation of the TMD in terms of a FE model is discussed, as well as the chosen design variables, constraints, and fitness functions definition. Moreover, the criteria to identify the optimal solution is derived, based on the Utopian Point Method [39]. Finally, a validation study is performed to examine the accuracy of the framework implemented.

Chapter 6 discusses the application of the framework to Case Study I, where the target structure is a prototype of a large Earth-based radio antenna structure. The structural efficiency of the antenna is assessed by the calculation of the deviation angle between the primary and secondary reflectors, therefore being the target of the study for minimization by implementing multiple-TMD passive control devices. The results for the inclusion of multiple-TMD for the three different placement configurations are presented and discussed.

Chapter 7 presents the use of the framework to minimize the aeroelastic response of a $12 \mathrm{~m}$ VLBI antenna, by means of the attachment of a single-TMD in the secondary reflector. Results are presented for different atmospheric disturbance analyzed in the frequency and time-domain, concurrently optimizing multiple operational scenarios of the antenna. The optimal constitutive parameters of the TMD are discussed and compared among the operational cases.

The conclusions, limitations of the analyses presented in the thesis and the recommendations for future work with applications are presented in Chapter 8 . 


\section{Chapter 2: Literature review}

\subsection{General aspects of TMD systems}

A TMD device is used in structures with the intent to attenuate their dynamic responses to different excitations. Their main application in the literature is more related to civil engineering, where they are employed to mitigate the vibration responses of structures for the comfort of the structural occupant's, or to increase the fatigue life, due to the minimization of amplitudes of oscillations [40]. For aerospace structures, their use is mainly related to increasing the structural performance under different operational conditions, thus allowing augmentation of stability and precision [41].

To achieve the desired attenuation effect, TMD systems can assume a variety of different configurations. The fundamental topology of a TMD is based on a SDOF model of a mass-springdamper, therefore being able to dissipate energy from a structure that it is attached to [42]. In order to properly operate, the natural frequency of the TMD is tuned to a particular target structure frequency, such that when excited at that frequency, the TMD resonates out of phase with the structural motion. This effect results in the dissipation of mechanical energy, by means of the resonator inertia force that acts upon the target structure. In this way, the designer should search for the constitutive parameters of the TMD that allows one to match its natural frequency with the target structure's frequency of interest, therefore characterizing a tuning process of the TMD system [42]. The outcome of tuning a TMD is usually the reduction in amplitude of response at the specific tuned frequency. However, for other values it is possible to observe an increase in the structural response, hence creating two coupled and greatly damped mode shapes from the initial single lightly damped peak [43]. 
The search for constitutive parameters of TMD that best attenuate the mechanical response of a structure is generally approached as an optimization problem, such that the selected parameters are able to maximize the structural performance, based on specified objective functions [44]. For simple models, it is possible to derive closed form analytical expressions that relate the mass ratio (ratio of the TMD mass to the target structure one), damping ratio of the target structure and the TMD, and tuned frequency ratio (ratio of the TMD frequency to the target structure one). For more complex models, the Finite Element (FE) method can be utilized to estimate the response at desired structural nodes [45]. The objective function, or performance criteria, is usually taken as the response level of the structure, which can be expressed by the quantities of displacement, velocity or acceleration, and the minimization of the TMD mass inclusion in the target structure.

Different configurations of TMD can provide distinct performance limitations, such as spatial limitations of the target structure, robustness to change in structural stiffness, cost of implementation and life cycle of the TMD system [42]. The following section of this chapter will discuss different TMD systems present in the literature, as well as their design strategies.

\subsection{Passive TMD devices}

The well-known distinctive characteristic of passive systems is related to not requiring an external source of power in order to effectively operate. When this concept is applied to TMD systems, it means that the given configuration does not encompass active actuators, linked with a feedback control method. Hence, the stability of passive TMD systems is not a concern, when properly designed, and they are essentially based on mechanical motion and energy transfer. Those characteristics make them suitable for long term applications where minimal maintenance is necessary. 
The major drawback of passive TMD devices relies on the lack of attenuation efficiency in detuning conditions [46]. As previously mentioned, the TMD devices are highly dependent on the tuning frequency, in order to minimize amplitudes of oscillations of a target structure. Outside of the small range of frequency band tuning of the TMD, the attenuation capability is compromised, even for minor deviations. Therefore, the efficacy of TMD systems is determined by the accuracy of the initial tuning provided, and whether the target structure presents any subsequent detuning behavior [47].

Nevertheless, even though with the existence of this limitation, passive TMD systems are frequently used due to the low cost of implementation/maintenance, and excellent performance when properly tuned [48]. Moreover, because of the passive nature of this vibration control devices, no external energy source is necessary for its utilization after they are installed in a structure. Two configurations that are frequently implemented are the translational (single-TMD and multiple-TMD) and pendular-TMD devices.

\subsubsection{Translational TMD systems}

Translational TMD devices essentially depends on the linear displacement of a seismic mass for one or more DOF. The mass rests on bearings or rollers, which allows its lateral translational movement with respect to a fixed floor or support. The springs and the dampers attached to the mass permits the out-of-phase motion of the TMD with respect to the floor beam. This configuration can be similarly employed for a bidirectional motion or multiple axis motion, such that vibration in orthogonal directions can be suppressed simultaneously. The first single-TMD with a translational behavior was implemented by Frahm in 1909 [49], where the application was to control the rolling motion of ships by means of an undamped mass-spring absorber. Vibration 
solutions utilizing translational TMD systems have been employed in several large scale structures for over 40 years, such as in the CN Tower (Toronto, Canada), John Hancock Tower (Boston, USA), Chiba Port Tower (Chiba, Japan) [48]. Some general characteristics of the design of singleTMD systems that have been investigated and concluded by various researchers, such as Connor [42], are as follows:

1. The detuning from the optimal value of the stiffness parameter in the TMD often has a significant influence in the performance of the single-TMD device

2. On the other hand, the detuning from the optimal value of the damping parameter in the TMD often has a marginal influence in the performance of the single-TMD device

3. The characteristic damping of the target structure has a crucial effect on the optimal value of the stiffness from the TMD device, however, marginal influence on its optimal value for the damping coefficient

In Ma et al. [34] paper, a graphical design of the MDOF-TMD is introduced, yielding capabilities of attenuating multiple mode shapes within a structure subjected to dynamic excitations. The concept behind the method relies on determining the freedoms and constraints of the TMD by using geometric patterns, such as orientations and directions for displacement or rotation (i.e. motion), with quantitative parameters to characterize the structural design of the resonator. The proposed flexure based MDOF-TMD allows motion in three planar DOF, where the stiffness and damping are controlled by means of a connection with cantilever beams in the model, where the components, design dimensions and manufactured structure are respectively depicted. Similar to this concept presented, Meng et al. [36] introduced a concept of translational TMD with planar 2DOF of actuation, based on the flexural behavior of cantilever beams acting as a spring-damper model. 
The inclusion of TMD systems is also capable of attenuating aeroelastic responses based on aerodynamic turbulence induced by gust loads. Applied to wind turbine structures, Zhang and Høeg [50] enhanced the attenuation capabilities of a simple translational TMD by including a rotational inertia double TMD (RIDTMD) in the model, in order to damp in-plane vibrations of floating offshore wind turbines. With the inclusion, it is possible to introduce an extra-resonance to the model. Chen et al. [28] compared two configurations of TMD devices, being a single and multiple inclusions, applied to attenuate the aeroelastic response of offshore wind turbines. The study concluded that the control efficiency of the single-TMD device is superior to the multipleTMD, when large masses are introduced in the structure. Del Campo et al. [51] demonstrated in a very interesting statistical study that by including passive damping systems in land based wind turbines, such as optimally tuned TMD devices, a fragility reduction of $80 \%$ is reached under severe atmospheric turbulence excitations, such as cyclone-induced winds, and is considerably lower for seismic excitations. Regarding the mitigation of flutter oscillations in bridges, Boonyapinyo et al. [52] investigated a model of single-TMD and multiple-TMD, using the modal approach, demonstrating that for the correct tuning of the constitutive parameters the buffeting response can be effectively reduced. Lin et al. [53] presented a concept of TMD capable of simultaneously reducing torsional and vertical buffeting of long-span bridges. The TMD system is tuned to the first two frequencies of the structure, related to the flexural and torsional mode shapes. Other applications of translational TMD devices for mitigation of aeroelastic response are presented in the works of $\mathrm{Xu}$ et al. [54], Bi et al. [55], Jiang [56], Ubertini et al. [57], Kwok and Samali [58].

\subsubsection{Multiple-TMD systems}


In essence, multiple-TMD systems utilize several single-TMD devices in order to control the vibration response of structures. The main difference when compared to a single-TMD system is that instead of using a single large mass to result in the out-of-phase motion in a specific frequency, multiple smaller auxiliary masses are included in the target structure, and are placed in designated locations [59]. The design of multiple-TMD systems allows a better control efficiency in detuning conditions, when compared to single-TMD systems, due to the fact that various DOF and resonances are introduced in the target structure [60].

Another advantage of multiple-TMD systems lies on the geometrical characteristics of the target structure, for example, sometimes there is not enough space to include a large mass, as in a singleTMD system. The inclusion of multiple-TMD overcomes this space constraint, as it is possible to distribute the auxiliary masses throughout the structure, allowing higher mass ratios to be utilized [45]. Furthermore, Sun et al. [61] demonstrated if the total equivalent mass of a single-TMD is subdivided into several smaller masses, as in a multiple-TMD configuration, it is possible to achieve similar levels of attenuation in the dynamic response.

The fundamentals of design of multiple-TMD systems are essentially to systematically tune the natural frequencies of each TMD individual around the target natural frequency of the structure. This interval of tuning is important in order to increase the bandwidth of actuation of the multipleTMD set. Another approach would also tune the TMD systems according to multiple structural modal frequencies [42]. Yamaguchi and Harnpornchai [37] presented an analytical study investigating the optimal range of frequency tuning of multiple-TMD devices, as well as the optimal number of resonators to be included. Furthermore, as discussed in the work of Elias et al. [22], and Debnath et al. [29], multiple-TMD can be utilized for control of multiple mode shapes 
of the target structure, such that each TMD device is tuned to a specific frequency, and placed in a location of highest amplitude of dynamic response.

Similarly as in the case of the single-TMD system, for a multiple-TMD configuration to work at its best efficiency, the constitutive parameters must be properly optimized. Casalotti et al. [62] investigated the optimal properties of a system of multiple hysteretic-TMD to control multi-mode flutter in long-span suspension bridges, such that the constitutive parameters of the TMD presented a characteristic of nonlinearity. Lin and Cheng [63] studied the performance of multiple-TMD to suppress the buffeting response of long-span bridges, demonstrating that this configuration presents superior attenuation capabilities when compared to a single-TMD device for a greater frequency bandwidth of gust excitation.

\subsubsection{Pendular-TMD systems}

The pendular-TMD configuration presents a great advantage with respect to the translational TMD, where the design of bearings and rail systems is substituted by the use of a network of supporting cables, which allows the mass to pivot about a fixed point [42]. The use of a bearing support for the TMD is expensive and can be subjected to wear and fatigue over the lifespan of utilization of the TMD system, thus the solution of a pendular-TMD allows the reduction of implementation costs and lifetime of the control device. Due to those characteristics and the proven efficiency to attenuate natural hazards, such as wind gust loads and seismic excitations, the pendular-TMD configuration has been extensively utilized in tall civil structures, for example as in the Taipei 101 building [64].

The simplest model of a pendular-TMD is essentially represented as a linear pendulum with small angles of oscillation. For this configuration, the behavior is practically identical to the translational 
TMD, where it can be modelled with an equivalent mass, spring and damping coefficient, as well as the maximum rotation amplitude [42]. Soltani and Deraemaeker [65] presented a study to investigate optimum parameters of pendular-TMD systems considering multiple combinations of response and excitation conditions, such as random loads, base excitation, among others. Roffel and Narasimhan [66] studied the performance of installed pendular-TMD systems, establishing a framework to conduct their condition assessment in-service, where the frequency and damping ratio are analyzed.

\subsubsection{Tuned Liquid Column Damper systems}

Tuned Liquid Column Damper (TLCD) systems utilize a reservoir with a fluid, as a replacement for the fixed mass, where commonly water is employed. The fluid is usually in a tank with a slittedgate placed in the middle of it, or in a tube with an orifice in the horizontal segment. The principle of operation is that the sloshing motion of the fluid presents an out-of-phase motion with respect to the target structure, hence counter-balancing the effect of mechanical vibrations.

Due to the high-nonlinearity characteristic of the sloshing motion of the fluid, the design of TLCD systems is relatively complex, requiring extensive mathematical formulation to obtain an algorithm to tune the frequency of operation of the resonator. Tuong et al. [67] presented a numerical study investigating the optimal parameters of a TLCD, such as cross-sectional area and lengths of the reservoir, head loss coefficient, applied to high-rise buildings subjected to wind gust excitations. Neto [68] developed a mathematical procedure to model the sloshing motion of the TLCD device in terms of a dynamic mass-spring-damper system, implementing a parametric optimization framework to minimize the dynamic response of a simple 1-DOF structure. Other 
relevant work related to the design of TLCD systems are described in Zhang et al. [69], Kim and Adeli [70], and Love and Tait [71].

\subsection{Active-TMD devices}

Differently from passive systems, active-TMD control devices resort upon an external energy source to operate, such as using an actuator. Due to the use of controllers, active-TMD systems also require the use of a closed feedback control loop, where sensors are utilized to measure conditions such as displacement, acceleration, or velocity as the feedback response. The common components for the design of an active-TMD are an actuator, the auxiliary mass, and sensors to create the closed feedback loop. They are usually implemented in structures subjected to intense dynamic loading.

The principle of operation of an active-TMD consists in the active motion control of the auxiliary mass of the TMD, which should damp the response of the target structure where the control device is attached to [72]. There are a few advantages related to the use of active-TMD systems. One of them is related to the consistency in the efficiency during detuning conditions since they can be counter-balanced by means of the feedback control loop [73]. This is a significant improvement when compared to passive TMD systems, which present lower efficiency when the structure is excited in frequencies different from what the TMD is designed for. Another characteristic is the excellent capability of controlling transient behavior of structures, such as excited by impact loads like earthquakes or wind gust loads [74].

The drawbacks of utilizing active-TMD are related to implementation costs of this configuration due to the high-complexity of its design, manufacturing, and precise instrumentation to be used in the feedback control loop. The use of a power source can also greatly contribute to the operation 
costs of this solution. A way around is to utilize hybrid systems [42], which work mainly as a passive TMD system, but after overcoming a specific detuning threshold, the active feedback loop is triggered. An example of a hybrid TMD system is investigated in the work of Engle et al. [75], where the control device is utilized for vibration isolation of floor slabs in the event of an earthquake, being able to reduce the vibration levels up to $40 \%$ in magnitude for any condition of excitation inputs.

\subsection{Semi active-TMD devices}

Semi active-TMD devices present mixed characteristics of passive and active-TMD systems, yet presenting significantly lower energy consumption when compared to active systems [76]. In essence, the semi active-TMD solutions provide active control of constitutive parameters of the resonator, such as stiffness or damping, resulting in the maintenance of the system's stability. Therefore, by allowing the active control of TMD properties while consuming low amounts of energy, the semi active configurations provide greater control capability than passive systems, while attenuating the drawbacks of implementing an active control solution.

There are a plethora of methods to implement semi active-TMD control to structures. Colherinhas et al. [44] presented the design of a semi active pendular-TMD for attenuating mechanical vibrations in offshore wind turbines, where the length of the pendulum device can be changed in order to tune the frequency of operation of the TMD, such that it matches the predicted operational scenario. Nagarajaiah and Sonmez [77] investigated the performance of single and multiple semi active TMD with variable stiffness and damping coefficients of a shear-building under several operation scenarios. Chatziathanasiou et al. [78] proposed a novel semi active-TMD configuration, by utilizing shunted piezoelectric circuits for robust control of a 1-DOF structure model. Weber 
[79] designed a real-time controlled magnetorheological TMD for mitigation of structural vibrations. The magnetorheological damper contains a fluid that is responsive to the change of the magnetic field surrounding it, hence allowing to change its viscosity accordingly. The conclusions among the papers presented indicate that the use of semi active solutions provide greater performance than passive systems for excitation detuning conditions in the target structure.

\subsection{Optimization of TMD systems}

As previously presented, in order to operate at their maximum efficiency, the constitutive parameters of passive TMD systems have to be correctly tuned, usually by means of optimization algorithms or analytical solutions, in the case of simpler structures [42]. This section will focus on reviewing some relevant optimization methods present in the literature for the TMD configurations previously presented.

Hoang et al. [80] proposed closed-form equations for the optimization of constitutive parameters of translational conventional TMD applied to long span bridges under seismic loads, modelled with the Kanai-Tajimi spectrum. The main conclusion of the work was that the optimal TMD set assumes low frequencies values, as well as a direct proportionality between the damping ratio and mass ratio of the resonator. Lavan [81] implemented a multi-objective optimization procedure for designing a TMD system considering either a base or external load excitation, presenting two approaches for the problem. The first is essentially the use of MDOF system formulation of TMD, while the second considers the modal approach to the problem. The objectives minimized are the acceleration response of the structure, the TMD stroke length and the total mass inclusion. Bekdaş et al. [82] implemented a novel approach to design TMD parameters, by means of a Bat Algorithm, considering conventional design variables such as mass, tuning frequency and damping ratio, 
showing that it can be more efficient in finding the best solution when investigating a shear building dynamic. The Bat algorithm is a bio-inspired metaheuristic global optimization algorithm based on echolocation phenomena of microbats, where it utilizes parameters related to selective search to determine the global solution, such as changing frequency, loudness and pulse emissions, which intensified by a random walk [83]. Still related to metaheuristic algorithms, Nigdeli et al. [84] investigated the optimization of TMD parameters using a flower pollination algorithm, where the optimal TMD device is found for near-fault excitations using multiple configurations of impulse motions. Liu et al [85] utilized the genetic algorithm optimization method to design TMD, considering the stroke of the resonator as a constraint for the optimization problem. Batou and Adhikari [86] investigated the optimal properties of a viscoelastic TMD using the fixed points theory, where an analytical formulation that considers the Biot model was derived. Khatibinia et al. [87] determined the optimal properties of a conventional TMD by implementing the improved gravitational search algorithm, where the objective function to be minimized is taken as the root mean square of different DOF in a benchmark structure.

As for multiple-TMD configurations, Mohebbi et al. [88] implemented a framework to design the multiple resonators applied to a ten-story shear building structure, based on genetic algorithms, where the objective function to be minimized is the maximum structure's response in the time domain when subjected to seismic loads. They concluded that the performance of multiple-TMD devices increases when the mass inclusion ratio decreases. Debnath et al. [29] developed a strategy to optimize the location and parameters of multiple-TMD, based on the work of Yamaguchi and Harnpornchai [37], by means of the modal frequency response function of truss bridges, specifically the Saraighat Bridge. When considering general loading excitations, excellent attenuation of mode shapes along transverse and vertical directions were obtained. 


\section{Chapter 3: Theoretical framework part I - Numerical modelling of aerospace structures with multiple-tuned mass dampers}

\subsection{Introduction}

In this chapter, the formulation of both the single-TMD and the multiple-TMD are presented in the global equation of motion (EOM) of the structural FE model considering the mass, stiffness and damping matrices. In sequence, the aeroelastic equation of motion is derived with the modified FE constitutive matrices, considering the gust models given by a PSD function modelled with the statistical model DS and the TDG. Then, the general formulation of the TCLD is proposed, such that different degrees of freedom (DOF) can be tuned in phase to account for a net displacement when analyzing a response signal in the frequency domain.

\subsection{Structural model with TMD}

A structure modeled with FE can be discretized into a specific number of DOF that are connected between elements and nodes. The properties of the elements are defined by means of the mass, stiffness and damping constitutive matrices, which can be assembled to obtain the global arrangement of a structure [89].

Considering a generalized structure with $N$ number of DOF and $K$ number of attached TMD, as depicted in Figure 3.1, each auxiliary control device included can be modelled as local inclusions of mass, stiffness and damping values at the structure's nodes, which modifies the dynamic global constitutive matrices of the FE model. The equation of motion of the system shown in Figure 3.1, is written as in Equation (3.1), 


$$
\widetilde{\mathbf{M}} \ddot{\mathbf{u}}(t)+\widetilde{\mathbf{C}} \dot{\mathbf{u}}(t)+\widetilde{\mathbf{K}} \mathbf{u}(t)=\widetilde{\mathbf{P}}(t)
$$

where $\widetilde{\mathbf{M}}, \widetilde{\mathbf{C}}$ and $\widetilde{\mathbf{K}}$ are, respectively, the constitutive global FE matrices of mass, damping and stiffness of the system modified by the inclusion of the multiple-TMD; $\mathbf{u}(t)=\operatorname{col}(\mathbf{x}(t), \mathbf{y}(t))$ is the nodal displacements column vector, that concatenates the displacement DOF of the structure's nodes, named as $\mathbf{x}(t)$, with the multiple-TMD's DOF, named as $\mathbf{y}(t)$. The notations $\left({ }^{\circ}\right)$ and $\left({ }^{*}\right)$ indicate the first and second derivatives with respect to time, $t$, respectively, hence denoting the velocity and acceleration vectors for each DOF in the system. Moreover, $\widetilde{\mathbf{P}}(t)=\operatorname{col}(\mathbf{P}(t), \mathbf{0})$ is the column vector that defines external forces applied to the structure's nodes. As it will be discussed further in the text, the variable of external forces $\widetilde{\mathbf{P}}(t)$ is separated into two categories, the first being force functions containing non-aerodynamic excitations, and the second being the aerodynamic disturbances applied to the structure.

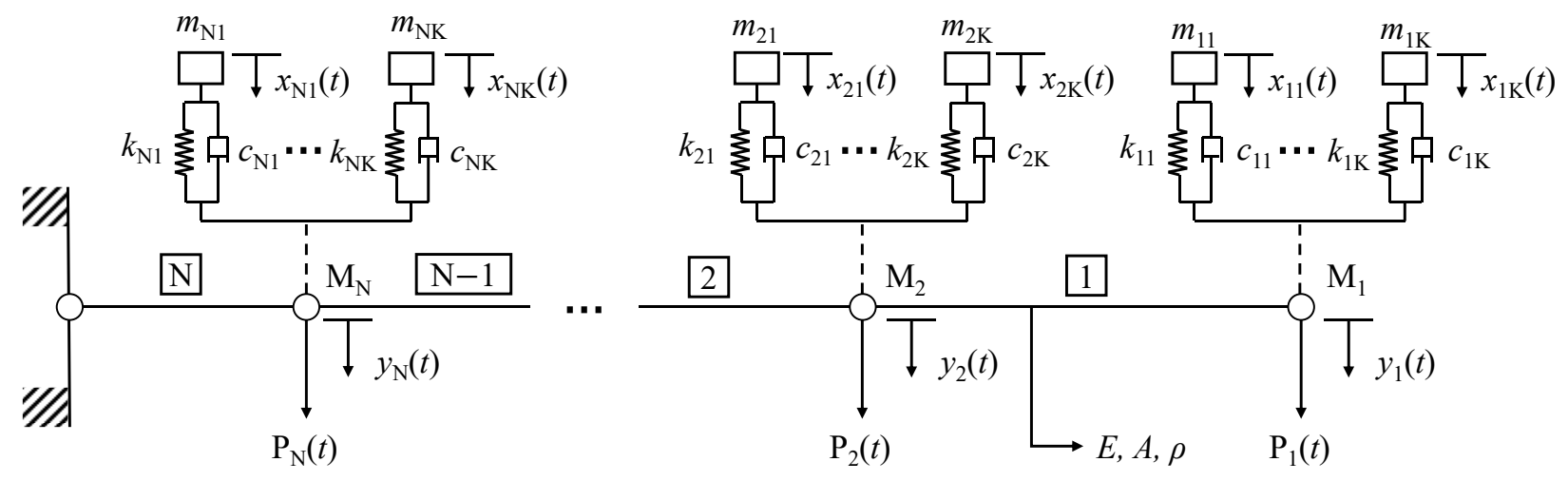

Figure 3.1: FE model of a generic structure with the inclusion of multiple-TMD

It is of interest to understand how the multiple-TMD modifies the global FE matrices. The general form of the $\widetilde{\mathbf{M}}$ matrix, from Equation (3.1), is described by Equations (3.2.a) and (3.2.b), 


$$
\widetilde{\mathbf{M}}=\left[\begin{array}{cc}
\mathbf{M} & \mathbf{0} \\
\mathbf{0} & \mathbf{m}
\end{array}\right]
$$

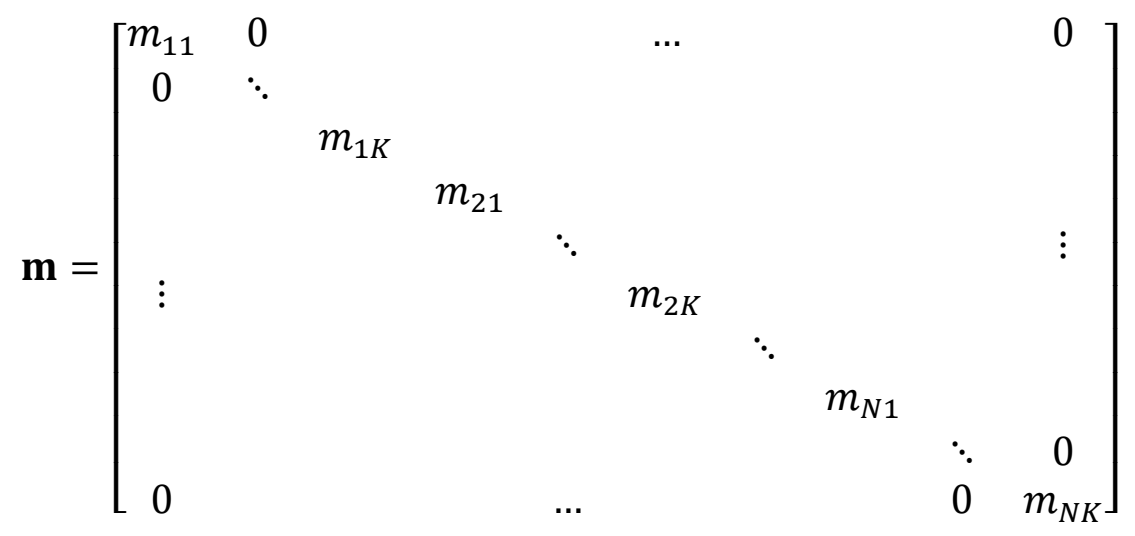

where $\widetilde{\mathbf{M}}$ is the block diagonal matrix assembled with $\mathbf{M}$, which is the material mass matrix of the system, and $\mathbf{m}$, representing the individual mass inclusions of each TMD considered, i.e., the values for $m_{i j}$, where $i=1, \cdots, N$ and $j=1, \cdots, K$. The modified global stiffness matrix, $\widetilde{\mathbf{K}}$, can be written as in Equations (3.3.a) to (3.3.d),

$$
\begin{aligned}
& \widetilde{\mathbf{K}}=\left[\begin{array}{cc}
\mathbf{K}+\overline{\mathbf{k}} & \overline{\overline{\mathbf{k}}} \\
\overline{\overline{\mathbf{k}}}^{T} & \mathbf{k}
\end{array}\right] \\
& \overline{\mathbf{k}}=\left[\begin{array}{ccc}
k_{11}+k_{12} \cdots+k_{1 K} & \cdots & 0 \\
\vdots & \ddots & \vdots \\
0 & \cdots & k_{N 1}+k_{N 2} \cdots+k_{N K}
\end{array}\right] \\
& \overline{\overline{\mathbf{k}}}=\left[\begin{array}{cccccccccccc}
-k_{11} & -k_{12} & \cdots & -k_{1 K} & 0 & 0 & 0 & 0 & & \cdots & & 0 \\
0 & 0 & 0 & 0 & -k_{21} & -k_{22} & \cdots & -k_{2 K} & & \cdots & & 0 \\
0 & 0 & 0 & 0 & 0 & 0 & 0 & 0 & \ddots & \cdots & & 0 \\
\vdots & & & & & & & & & & \vdots \\
0 & & & & \cdots & & & 0 & -k_{N 1} & -k_{N 2} & \cdots & -k_{N K}
\end{array}\right]
\end{aligned}
$$




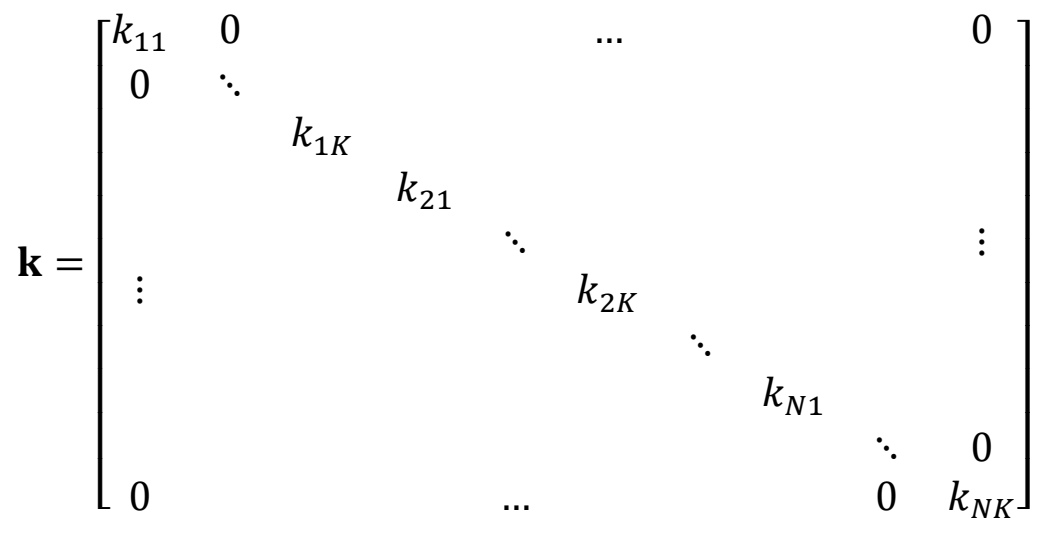

where $\mathbf{K}$ is the global material stiffness matrix of the structure, which is assembled with the stiffness compliance matrices of the multiple-TMD inclusion, defined as $\overline{\mathbf{k}}, \overline{\overline{\mathbf{k}}}$ and $\mathbf{k}$. The individual values of stiffness for each TMD are defined as $k_{i j}$, where $i=1, \cdots, N$ and $j=1, \cdots, K$. The notation $\left({ }^{T}\right)$ indicates the transposition operation of a matrix. Analogous to the stiffness matrix assembling process, the modified global damping matrix $\tilde{\mathbf{C}}$ can be formulated as in Equations (3.4.a) to (3.4.d),

$$
\begin{aligned}
& \tilde{\mathbf{C}}=\left[\begin{array}{cc}
\mathrm{C}+\overline{\mathbf{c}} & \overline{\overline{\mathbf{c}}} \\
\overline{\overline{\mathbf{c}}} & \mathbf{c}
\end{array}\right] \\
& \overline{\mathbf{c}}=\left[\begin{array}{ccc}
c_{11}+c_{12} \cdots+c_{1 K} & \cdots & 0 \\
\vdots & \ddots & \vdots \\
0 & \cdots & c_{N 1}+c_{N 2} \cdots+c_{N K}
\end{array}\right] \\
& \overline{\overline{\mathbf{c}}}=\left[\begin{array}{cccccccccccc}
-c_{11} & -c_{12} & \cdots & -c_{1 K} & 0 & 0 & 0 & 0 & & \cdots & & 0 \\
0 & 0 & 0 & 0 & -c_{21} & -c_{22} & \cdots & -c_{2 K} & & \cdots & & 0 \\
0 & 0 & 0 & 0 & 0 & 0 & 0 & 0 & \ddots & \cdots & & 0 \\
\vdots & & & & & & & & & & & \vdots \\
0 & & & & \cdots & & & 0 & -c_{N 1} & -c_{N 2} & \cdots & -c_{N K}
\end{array}\right]
\end{aligned}
$$




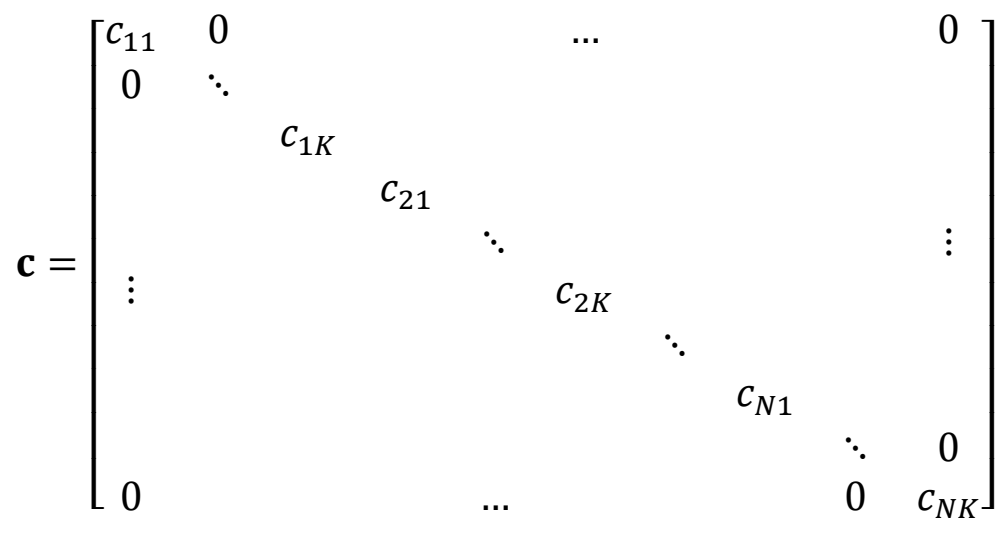

where $\mathbf{C}$ is the global material damping matrix of the structure, which is assembled with the damping compliance matrices of the multiple-TMD inclusion, defined as $\overline{\mathbf{c}}, \overline{\mathbf{c}}$ and $\mathbf{c}$. The individual values of damping for each TMD are defined as $c_{i j}$, where $i=1, \cdots, N$ and $j=1, \cdots, K$.

From Equation (3.1), the response $\mathbf{u}(t)$ can be expressed as a linear combination of harmonic motions functions, $\mathbf{q}_{i}(t)$, with distinct deformation patterns, named mode shapes $\boldsymbol{\varphi}_{i}$, that are associated with discrete values of frequency, also known as natural frequencies $\omega_{n_{i}}$ [90]. The mode shapes and natural frequencies of the structure with multiple-TMD can be found by means of the formulation of an eigenvalue problem considering the undamped free vibrating system. These relations are given by Equations (3.5.a) and (3.5.b),

$$
\begin{gathered}
\left(\widetilde{\mathbf{K}}-\omega_{n_{i}} \widetilde{\mathbf{M}}\right) \boldsymbol{\varphi}_{i}=\mathbf{0} \\
\mathbf{u}(t)=\sum_{i=1}^{R} \boldsymbol{\varphi}_{i} \mathbf{q}_{i}(t), \quad R=N+K
\end{gathered}
$$


FE models of aerospace structures usually contain thousands of DOF, which often makes them to be computationally expensive to solve. In order to simplify the equation of motion for faster computation of solutions, given by Equation (3.1), the property of orthogonality between the mode shapes can be exploited in order to uncouple the DOF of the mass, stiffness, and damping matrices, such that those matrices become diagonal. After the normalization procedure of the matrices, they are now defined in the modal, or generalized, coordinates as presented in Equations (3.6.a) to (3.6.c),

$$
\begin{gathered}
\widetilde{\mathbf{M}}_{q}=\boldsymbol{\varphi}_{i}{ }^{T} \widetilde{\mathbf{M}} \boldsymbol{\varphi}_{j}=\left\{\begin{array}{cc}
0, & i \neq j \\
m_{q_{j}}, & i=j
\end{array}\right. \\
\widetilde{\mathbf{C}}_{q}=\boldsymbol{\varphi}_{i}{ }^{T} \tilde{\mathbf{C}} \boldsymbol{\varphi}_{j}=\left\{\begin{array}{cc}
0, & i \neq j \\
c_{q_{j},} & i=j
\end{array}\right. \\
\widetilde{\mathbf{K}}_{q}=\boldsymbol{\varphi}_{i}{ }^{T} \widetilde{\mathbf{K}} \boldsymbol{\varphi}_{j}=\left\{\begin{array}{cc}
0, & i \neq j \\
k_{q_{j},} & i=j
\end{array}\right.
\end{gathered}
$$

\subsection{Aerodynamic model}

When investigating the dynamic behavior of aerospace structures, it is pivotal to consider the appropriate aerodynamic model to determine the external excitations on the structure. In the literature, there are a plethora of methods that allow one to achieve excellent approximations for the computation of the distribution of aerodynamic forces, such as the Strip theory [91], the Vortex-Lattice method [92] or the Doublet-Lattice method (DLM) [93]. For this work, we will utilize the DLM to compute the unsteady Aerodynamic Influence Coefficients (AIC), defined in the frequency domain. 
The DLM is commonly used as a reference tool in the aerospace industry in order to determine the response of structures under unsteady aerodynamics. Its main advantage relies on the fact of the low computational cost and the integration with commercial software, such as MSC NASTRAN ${ }^{\circledR}$. However, this method presents limitations in terms of its approximations due to the use of linearized potential equations, therefore not describing essential effects for aerodynamic analysis, such as geometry thickness, fluid viscosity, or shockwave formation, often resulting in overprediction of lift in aerodynamic surfaces [94]. Considering the DLM limitations in estimating aerodynamic coefficients, it is a common procedure in the industry to match the steady results at zero frequency with a higher order model by using correction factors, such as computed with CFD analysis or validated with steady-state wind tunnel data, in order to guarantee a good compromise between performance and accuracy in the results [95].

A brief introduction about the method is presented, where the major focus will be to describe how the inclusion of multiple-TMD affects the formulation.

\subsubsection{Doublet-Lattice method}

In the DLM, the aerodynamic surfaces are divided into trapezoidal panels, that are also subdivided into boxes that define the lifting elements of the panels. An acceleration potential doublet of uniform, however undefined, magnitude is placed conventionally at the $1 / 4$ of the box chord. The aerodynamic grid point ( $k$-set, in collection) is defined exactly at the center of each box, in which the aerodynamic forces, $\mathbf{P}_{k}$, and displacements, $\mathbf{u}_{k}$, are calculated considering the existent pressure distribution. The control point ( $j$-set, in collection) to determine the downwash vector, $\boldsymbol{w}_{j}$, is defined at three-quarters of each box chord, in which the flow tangency Kutta condition must be satisfied [93]. 
The downwash vector $\boldsymbol{w}_{j}$ at the $j$-th point can be determined using two equations. The first relates the AIC matrix, $\mathbf{A}_{j j}$, and the normalized lifting pressure at the $j$-th point, $\boldsymbol{f}_{j}$. The second relates the displacements $\mathbf{u}_{k}$ at the $k$-th point with the substantial differentiation matrices, defined as $\mathbf{D}_{j k}^{1}$ and $\mathbf{D}_{j k}^{2}$. Those relations are given in Equations (3.7.a) to (3.7.c),

$$
\begin{gathered}
\boldsymbol{w}_{j}=\mathbf{A}_{j j} \boldsymbol{f}_{j} \\
\mathbf{P}_{k}=\mathbf{S}_{k j} \boldsymbol{f}_{j} \\
\boldsymbol{w}_{j}=\left[\mathbf{D}_{j k}^{1}+i k \mathbf{D}_{j k}^{2}\right] \mathbf{u}_{k}, \quad k=\frac{\omega b}{V_{\infty}}
\end{gathered}
$$

where $\mathbf{S}_{k j}$ is the integration matrix, and $k$ is defined as the dimensionless reduced frequency, which depends on the circular frequency $\omega$, the airfoil semi-chord $b$, and the free stream velocity $V_{\infty}$. Considering the intrinsic dependence between the AIC and the circular frequency, the load analysis is conveniently carried in the frequency domain, being able to transform again in the time domain by means of inverse Fourier transformations. However, it is possible to proceed with the analysis in the frequency domain by using the Time Consistent Loads and Displacements (TCLD) method, which will be presented in the following sessions.

By combining the Equations (3.7.a) to (3.7.c), one obtains the Force Aerodynamic Influence Coefficients matrix, defined as $\mathbf{Q}_{k k}$, and can be evaluated at the aerodynamic grid points as presented in Equation (3.8), 


$$
\mathbf{Q}_{k k}=\mathbf{S}_{k j}\left[\mathbf{A}_{j j}\right]^{-1}\left[\mathbf{D}_{j k}^{1}+i k \mathbf{D}_{j k}^{2}\right]
$$

The structural and aerodynamic grid sets generally do not coincide due to the independent creation of them. Therefore, to achieve the load and displacements transfer between one set to another, it is necessary to use an interpolation function, usually referred to splining. The splining matrix, $\mathbf{G}_{k d}$, can be obtained through a variety of techniques, such as surface splines or linear splines [96]. Linear splines are based on the unidimensional beam theory, allowing to couple the aerodynamic and structural grid for representing bending or torsional deflections. Surface splines are based on the infinite plate theory, allowing multiple deflection points within the area, providing a higher fidelity coupling between the aerodynamic and structural grid.

Hence, using the splining matrix, it is possible to use the AIC matrix to compute the change in aerodynamic forces due to structural deformations, that are evaluated in the structural grid and are defined by the matrix $\mathbf{Q}_{d d}$, by employing Equation (3.9),

$$
\mathbf{Q}_{d d}=\mathbf{G}_{k d}^{T} \mathbf{Q}_{k k} \mathbf{G}_{k d}
$$

To perform the classic aeroelastic analysis, it is necessary to convert the $\mathbf{Q}_{d d}$ matrix to the modal coordinates by means of the operation defined in the Equation (3.10).

$$
\mathbf{Q}_{q}=\boldsymbol{\varphi}_{i}^{T} \mathbf{Q}_{d d} \boldsymbol{\varphi}_{j}
$$

\subsubsection{Aeroelastic model}


The form of the aeroelastic equation of motion, evaluated according to a steady initial position, dependent in forced vibrations and the reduced frequency, that is modified with the inclusion of multiple-TMD, and expressed in the frequency domain is given by Equation (3.11),

$$
\left[-\widetilde{\mathbf{M}}_{q} \omega^{2}+i \widetilde{\mathbf{C}}_{q} \omega+\widetilde{\mathbf{K}}_{q}-\frac{1}{2} \rho_{a i r} V_{\infty}^{2} \mathbf{Q}_{q}\right] \mathbf{q}(\omega)=\widetilde{\mathbf{P}}(\omega)
$$

where $\rho_{\text {air }}$ is the air density, $\mathbf{q}(\omega)$ is the harmonic modal response in the frequency domain, and $\widetilde{\mathbf{P}}(\omega)$ is the modal forces vector in the frequency domain, obtained via Fourier transform of the modal forcing function in the time domain $\widetilde{\mathbf{P}}(t)$. The modal forcing function encompasses generalized non-aerodynamic nature forces, defined as $\widetilde{\mathbf{P}}_{\text {gen }}(t)$, and generalized aerodynamic excitations, defined as $\widetilde{\mathbf{P}}_{\text {aero }}(t)$. Those are given by Equations (3.12.a) to (3.12.c).

$$
\begin{gathered}
\widetilde{\mathbf{P}}(t)=\widetilde{\mathbf{P}}_{\text {gen }}(t)+\widetilde{\mathbf{P}}_{\text {aero }}(t) \\
\widetilde{\mathbf{P}}_{\text {gen }}(t)=\boldsymbol{\varphi}_{i}{ }^{T} \widetilde{\mathbf{F}}(t) \\
\widetilde{\mathbf{P}}_{\text {aero }}(t)=\frac{1}{2} \rho V_{\infty}{ }^{2}\left[\boldsymbol{\varphi}_{i}{ }^{T} \mathbf{G}_{k d}{ }^{T} \mathbf{S}_{k j} \mathbf{A}_{j j}\right] \boldsymbol{w}_{j}
\end{gathered}
$$

where $\tilde{\mathbf{F}}(t)=\operatorname{col}(\mathbf{F}(t), \mathbf{0})$ is the column vector of non-aerodynamic forces. As it will be presented in this chapter, there are several methods to model the downwash forcing function $\boldsymbol{w}_{j}$, such as the one-minus cosine TDG, in the time domain, or the PSD modelled with the DS, in the frequency domain, among others. 
The solution of Equation (3.11), in terms of the harmonic modal response $\mathbf{q}(\omega)$, represents the aeroelastic response of the structure subjected to an arbitrary excitation function. It can be expressed by a multiplication of a transfer function, $\mathbf{H}(\omega)$, with the modal forces vector $\widetilde{\mathbf{P}}(\omega)$, that is given by Equation (3.13).

$$
\mathbf{q}(\omega)=\frac{1}{\left[-\widetilde{\mathbf{M}}_{q} \omega^{2}+i \tilde{\mathbf{C}}_{q} \omega+\widetilde{\mathbf{K}}_{q}-\frac{1}{2} \rho_{a i r} V_{\infty}{ }^{2} \mathbf{Q}_{q}\right]} \widetilde{\mathbf{P}}(\omega)=\mathbf{H}(\omega) \widetilde{\mathbf{P}}(\omega)
$$

The modal response can also be obtained in the time domain by means of an inverse Fourier transform of $\mathbf{q}(\omega)$, given by Equation (3.14).

$$
\mathbf{q}(t)=\int_{-\infty}^{\infty} \mathbf{H}(\omega) \widetilde{\mathbf{P}}(\omega) e^{i \omega t} d \omega
$$

Using Equation (3.5.b) combined with Equation (3.14), one can obtain the displacements from the modal response in the time domain.

\subsubsection{Atmospheric wind disturbances}

Aerospace structures are constantly subjected to induced atmospheric disturbances and turbulences while in operation. Gusts are local disturbances that affect the pressure distribution around aerodynamic surfaces, hence generating additional loads that can potentially result in instability or excessive vibrations. Vertical or lateral gusts are created when a gust-induced velocity gradient is normal to the flow path, while head-on gusts are defined when the gust-induced velocity gradient is parallel to the flow path. 
The force generated by the gust is found from the turbulence wind-gust velocity, $\Delta v$, that is obtained by means of the formulation of a chosen gust model. The total wind velocity, $v$, is given by a combination of the mean velocity, $v_{m}$, and the turbulence wind-gust velocity, $\Delta v$, as in Equation (3.15).

$$
v=v_{m}+\Delta v
$$

One type of disturbance profile is the discrete gust [97,98], in which an isolated vertical disturbance creates a pulse-type profile in the excitation, such as the one-minus cosine model. The expression that models the spatial behavior of the one-minus cosine gust velocity, $\Delta v$, is given by Equations (3.16.a) and (3.16.b),

$$
\begin{gathered}
\Delta v\left(x_{g}\right)=\frac{v_{g 0}}{2}\left(1-\cos \frac{2 \pi x_{g}}{L_{g}}\right), \quad 0 \leq x_{g} \leq L_{g} \\
x_{g}=v_{m} t
\end{gathered}
$$

where $v_{g 0}$ is the peak gust disturbance or design gust velocity, $L_{g}$ is the gust length, $x_{g}$ is the position of the structure in the spatial coordinates of the gust with respect to a fixed origin, and $t$ is the respective measured time from the position $x_{g}=0$. By combining both Equations (3.16.a) and (3.16.b), one obtains the aerospace structure response in the time domain.

In order to model continuous turbulences within the atmospheric boundary layer, a good representative model is given by the statistical representation of the Davenport spectrum (DS), due to the fact that it takes into account the ground effects $[99,100]$. In this model, the wind gust is 
represented by a uniformly spread force acting on the lifting surface from a certain direction. The disturbance gust component is characterized by a random process defined by the DS formulation, which depends on the mean wind speed and terrain roughness parameters. It can be expressed by the Equations (3.17.a) to (3.17.c),

$$
\begin{gathered}
S_{D}(\omega)=L_{g} v_{m} \kappa \frac{\beta \omega}{\left(1+\beta^{2} \omega^{2}\right)^{4 / 3}} \\
\beta=\frac{L_{g}}{2 \pi v_{m}} \\
\kappa=\frac{1}{\left[2.5 \ln \left(\frac{Z}{z_{0}}\right)\right]^{2}}
\end{gathered}
$$

where $\kappa$ is the surface drag coefficient, $z$ is the height from the ground up to the center of the structure, $z_{0}$ is height of the terrain roughness, which is measured between $z_{0}=3.94$ to 11.81 in at Goldstone, California [101]. The wind-gust velocity, $\Delta v$, is found by applying a white-noise input to a fourth order filter that approximates the DS model $[102,103]$.

\subsection{Time Consistent Loads and Displacements formulation}

The computation of loads and displacements in aeroelasticity analysis are usually carried out in the frequency domain, due to the dependence on the system reduced frequency. To transform the response back to the time domain, via inverse Fourier transform, can be somewhat computationally expensive when dealing with iterative optimization algorithms [104]. Hence, intending to save computation time, it is of interest to implement a method to obtain the values of loads and 
displacements, for all the DOF considered, that are time consistent when determined in the frequency domain.

For that, two parameters have to be defined. The first is the critical frequency, $\omega_{\text {crit }}$, which defines the circular frequency of a certain DOF, measured in degrees per second. The second is the critical phase angle, $\varphi_{\text {crit }}$, which is the correspondent phase angle of the chosen critical frequency at the same DOF, measured in degrees. The critical frequency is usually selected according to the maximum amplitude of the frequency response function, given a selected frequency range, of a specific DOF considered in the model. With the values of both parameters determined, the critical time with respect to the reference DOF, $t_{c r i t}$, is then defined as in Equation (3.18).

$$
t_{\text {crit }}=\frac{\varphi_{\text {crit }}}{\omega_{\text {crit }}}
$$

The parameter $t_{\text {crit }}$ represents the reference instance that will allow all DOF to be time consistent between each other, therefore the outputted response between nodes can be effectively compared since the signal is now expressed in the time domain. Then, if we consider an arbitrary DOF quantity to become time consistent, named target station, the conversion can be expressed by Equation (3.19),

$$
U_{s t n, T C L D}=U_{s t n} \cos \left(\varphi_{c r i t}-\varphi_{s t n}\right)
$$

where $U_{S t n, T C L D}$ is the time consistent load or displacement for a specific station, $U_{s t n}$ is the outof-phase load or displacement of the same station, and $\varphi_{s t n}$ is the phase angle of the load or displacement determined from the frequency response function. The variables $U_{s t n, T C L D}$ can now 
be operated together for different DOF due to the application of the time consistence formulation. In the case of displacement responses, for example, the application of the TCLD formulation could be of interest to determine a net vector that represents the magnitude of the structure's deformation when excited with the critical frequency. As it will be presented in the further chapters, this concept will be part of the proposed optimization procedure. The Algorithm 3.1 presents the procedure to determine the time consistent data of a response in the frequency domain.

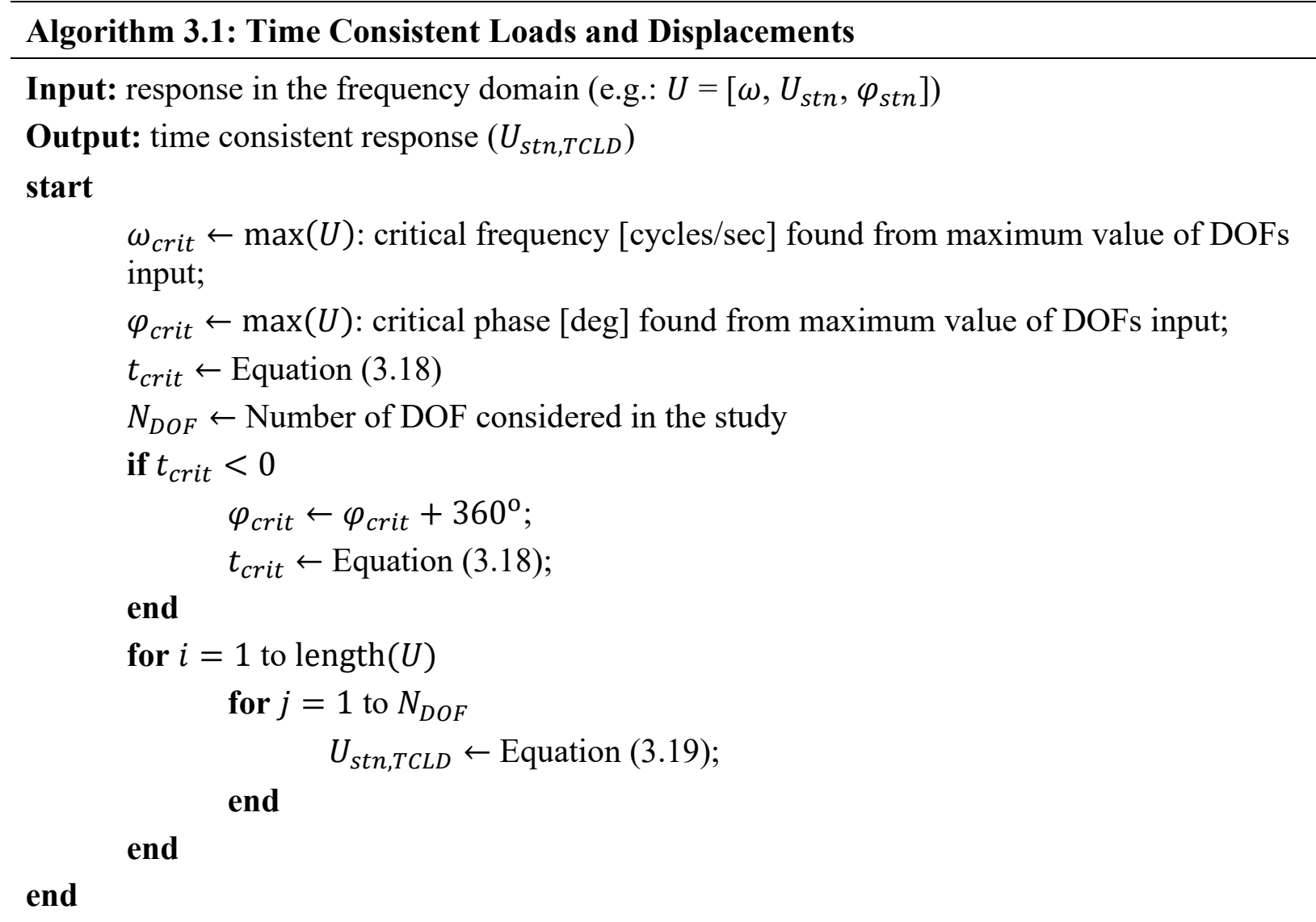




\section{Chapter 4: Theoretical framework part II - Optimization with genetic algorithms}

\subsection{Introduction}

Optimization is a search and comparison process of feasible solutions for a problem, where the best individual set is elected within a finite design space, based on imposed constraints or boundaries, and characterized as a minimization (or maximization) of an objective function. Such process can be of excessive complexity due to the usual high number of possibilities to be tested to solve a specific problem. Therefore, several techniques have been developed and are still under improvement in order to decrease the computation time of solutions, and to effectively determine global maxima or minima more efficiently.

Among the multitude of existent methods of optimization, one technique is the genetic algorithms, which is a mathematical abstraction of the biological evolution, initially proposed by Holland [105]. It is based in an analogy to the Darwinian evolution process though the mechanism of natural selection, and also relying on Mendelian genetics concepts to carry out the optimization. This chapter is dedicated to briefly describe the mathematical fundamentals behind the genetic algorithms, as well as common nomenclature utilized, and discuss its application in the scope of the present work.

\subsection{Optimization theory}

Mathematically describing, given a set $D \subset \mathbb{R}^{n}$ and a function $f: \Omega \rightarrow \mathbb{R}$, where $D \subset \Omega$, such that $f$ is minimized in the set $D . f$ it is given the name of objective function, and $D$ is the feasible set 
of the problem. The points belonging to $D$ are named as the design space. The problem is written as follows,

$$
\min f(x) \text {, subjected to: } x \in D
$$

In this way, a point $\bar{x} \in D$ globally minimizes $f$ if,

$$
f(\bar{x}) \leq f(x), \quad \forall x \in D
$$

Or locally minimizes $f$ in a neighborhood set $U$ of $\bar{x}$ such that,

$$
f(\bar{x}) \leq f(x), \quad \forall x \in D \cap U
$$

In other words, $\bar{x} \in D$ is a local minimizer of $f$ if there exists a $\varepsilon>0$ such that $\{\forall x \in D$ : $\|x-\bar{x}\| \leq \varepsilon\}$, i.e. for all $x$ in the neighborhood of $\bar{x}$, then $f(x) \geq f(\bar{x})$. It is noticeable that the concept of neighborhood, in this context, is similar to the limit. For an in-depth analysis of the mathematical formulation behind other concepts, such as infimum and supremum, it is recommended to read the works from Izmailov and Solodov [106], and Mendelson [107].

The optimal value of a function, defined as $\bar{f} \in[-\infty,+\infty]$, is expressed in the form of,

$$
\bar{f}=\inf \{f(x): x \in D\}
$$

which is the optimal value of the problem defined by Equation (4.1). From Equation (4.4), one realizes that a function can assume different global minimizers, however, the optimal value is the 
same for all the minimizers. Moreover, every global minimizer is also a local minimizer, but not the reciprocal. The Figure 4.1 depicts this concept.

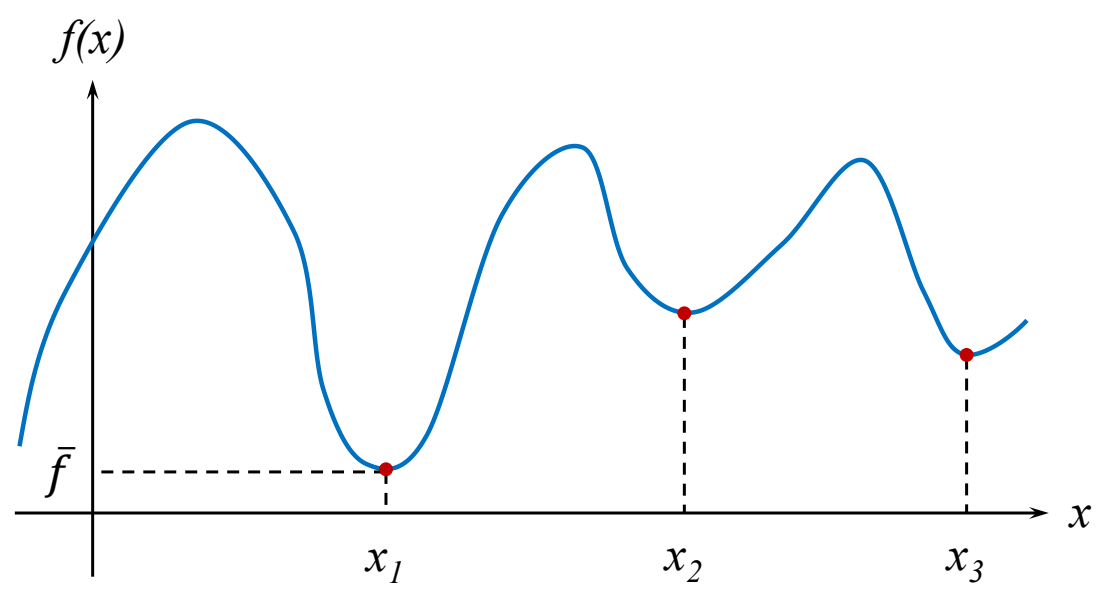

\section{Figure 4.1: Minimization of a function $f$, where $x_{1}$ is the global minimizer variable, and $\bar{f}$ is the optimal value}

The solution of optimization problems is essentially based into two approaches, being deterministic or stochastics. The first is usually evaluated utilizing derivatives of the objective functions, where a search algorithm determines critical points within the domain. The second is based on the evaluation of the objective function utilizing purely logic parameters to reach the solution. In other words, they are methods characterized by probabilistic analysis within the optimization process.

Stochastic approaches present an advantage with respect to the deterministic methods when the size of the search space is considerably large and rich in possible solutions. This generally occurs when a problem with several design variables is targeted. When using a stochastic method with evolutive attributes, it culminates in the concept of evolutive algorithms. Those characteristics are named heuristics, and essentially are part of processes of decision taking based on accumulated 
experiences. It will not be given a focus in this matter, besides mentioning that the genetic algorithms (GA) belong to the class of meta-heuristics methods. This approach efficiently searches the design space for global optimal solutions based on evolutive concepts of nature.

\subsection{Genetic algorithms}

The GA were initially introduced in the 60 's by Mitchell et al. [108], where the intention was to mathematically describe the adaptation phenomena observed in nature, such that their implementation in computational systems would be possible. Later, Goldberg [109] adapted the use of GA to apply in optimization problems, which resulted in its popularization within the research community, and the development of commercial software that use this tool, such as modeFONTIER [110], MATLAB® [111], Evolver [112], among others.

The natural selection mechanism is mathematically treated as a probabilistic process in GA. This means, in general terms, that the algorithm assumes that the characteristics of most adapted individuals tend to perpetuate over time under the constraint environment. Therefore, the GAs resort in this probabilistic selection to search for feasible solutions that are subjected to an ambient pressure, that is properly defined by the user. To achieve this result, random populations are generated, where the individuals carry chromosomes, and those are evaluated with an objective function, denominated fitness function, and are finally ranked with respect to one or more criteria [109]. The final step is usually related to the submission of the ranked individuals to the selection

mechanism, considering the genetic operators of crossover and mutation. The usual framework of a GA optimization follows the idea depicted in Figure 4.2. 


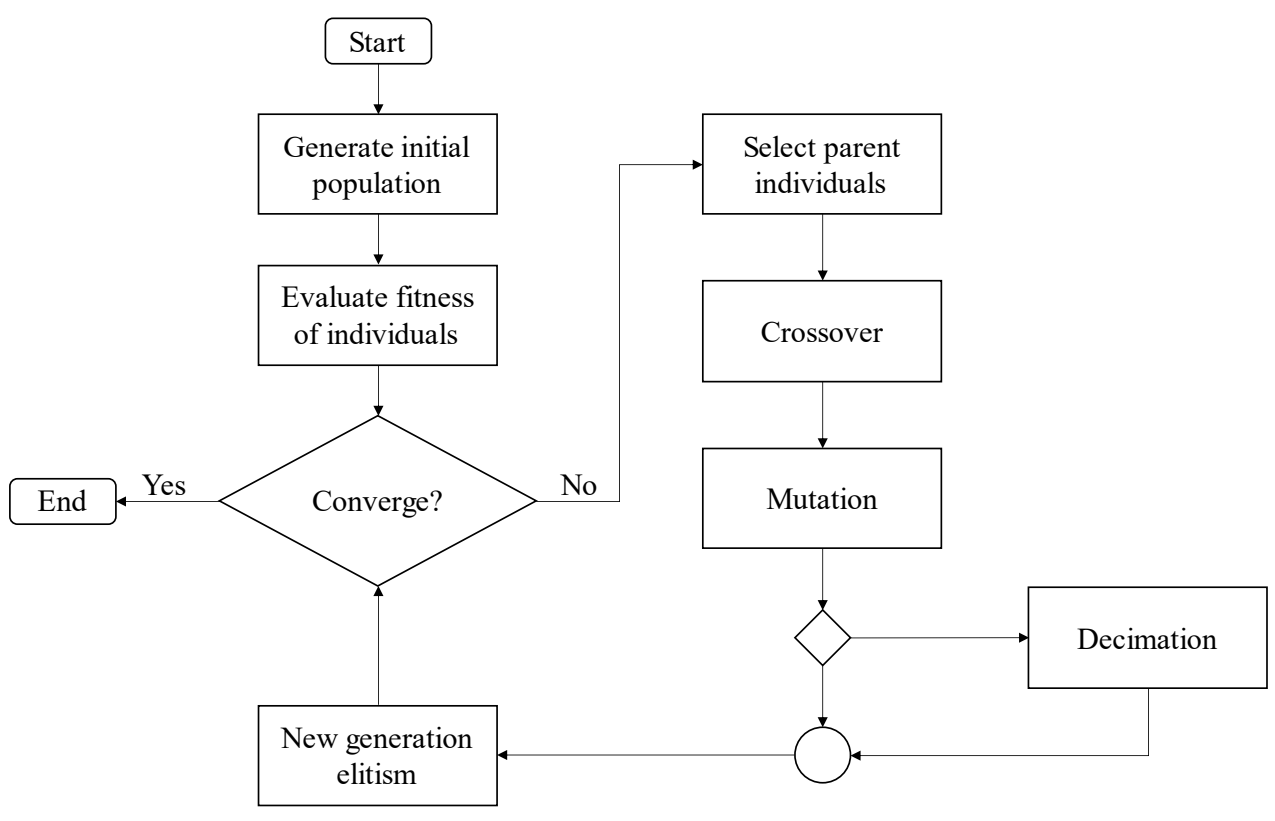

Figure 4.2: Flowchart of a standard GA

It is important to list some of the common nomenclature that is present in this context, as well as different existent codification methods that are commonly used to represent the genotype of an individual. Table 4.1 and Figure 4.3 summarize those concepts.

Table 4.1: Common nomenclature utilized in genetic algorithms context

Chromosome Individuals evaluated, or the solutions to the fitness function

Population A set of individuals

Nomenclature Gene A variable that defines the characteristics of an individual

Allele The value which the gene assumes

Generation

A counter that indicates the evolution of individuals, or the iterations number

Codification

Binary Each gene in the chromosome is identified as logical bits (zeros or ones)

Real Numbers of fluctuation points are utilized for representation 


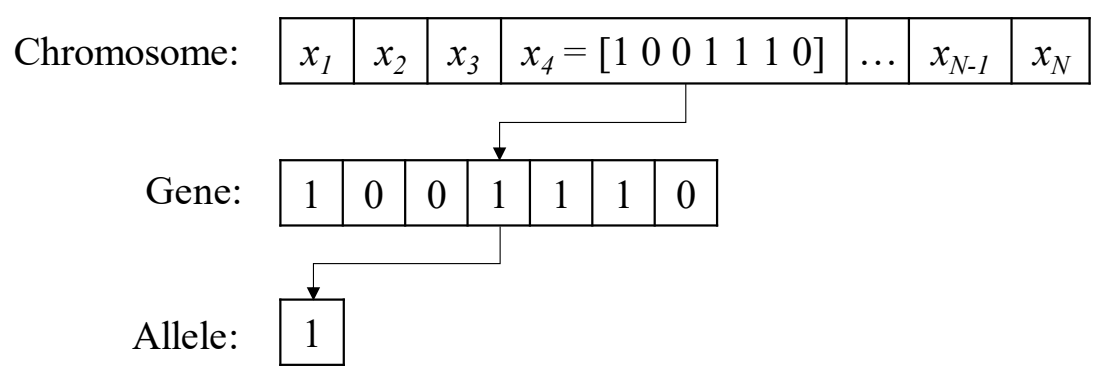

Figure 4.3: Components of a binary chromosome

\subsection{Initial population and objective function}

With the initialization parameters defined, i.e., codification type, problem constraints, maximum generation number, population size, the algorithm generates a random initial population within the established boundaries. This population corresponds to the first set of individuals which will be evaluated by the optimization objective function. In some cases, the initial population can be defined within a range of approximated solutions for the problem, which can considerably reduce the computation time [113].

The objective function, also named as fitness function, evaluates the candidate solutions of the problem, and it is the only connection with the real physics of the analysis. This function returns the value from a given individual under the problem constraints, which can be further utilized to define the rank of individuals and their respective aptitude for selection. The fitness function can be single or multi-objective, where in the second case it is intended to minimize or maximize concurrently two or more physical characteristics of the system, such as mass, safety factor, displacement, among others. 


\subsection{Genetic operators}

The genetic operators are the tools responsible for improving the aptitude of the generated individuals for each consecutive generation analyzed. They are necessary in a way that one can guarantee that the successful characteristics are effectively transmitted to further generations.

Moreover, they occur with a probabilistic behavior. Therefore, a probability of occurrence in the iterations of the GA is attributed, where two individuals (denominated parents) from a population are utilized to crossover and generate a new pair of individuals (denominated children). The mutation process occurs as a random change in the genetic material in one or more individuals of a same population. Those concepts related to the selection methods of individuals, ranking, crossover and mutation are discussed in the following sections.

\subsubsection{Individual selection}

After the generation of the initial population, and the evaluation of the individuals with the fitness function, it is necessary to determine which individuals will perpetuate to the next generation. A specialized algorithm selects the chromosomes with the best genetic characteristics, by means of stochastic decisions. The selection is based on different criteria, and some of the common strategies are the proportional, rank, and tournament selection.

\subsubsection{Proportional selection (Roulette - Wheel method)}

The proportional selection is a stochastic method where a section of an imaginary roulette-wheel is attributed to each chromosome [105]. Each section is proportional to the aptitude of the referred individuals by means of the objective function evaluation. The roulette-wheel is then rotated $N$ times, where $N$ is the number of individuals in the population. When the roulette stops, the 
individual is selected and used to participate in the further evolutive strategies of crossover and mutation. Figure 4.4 depicts the concept of the roulette-wheel method.

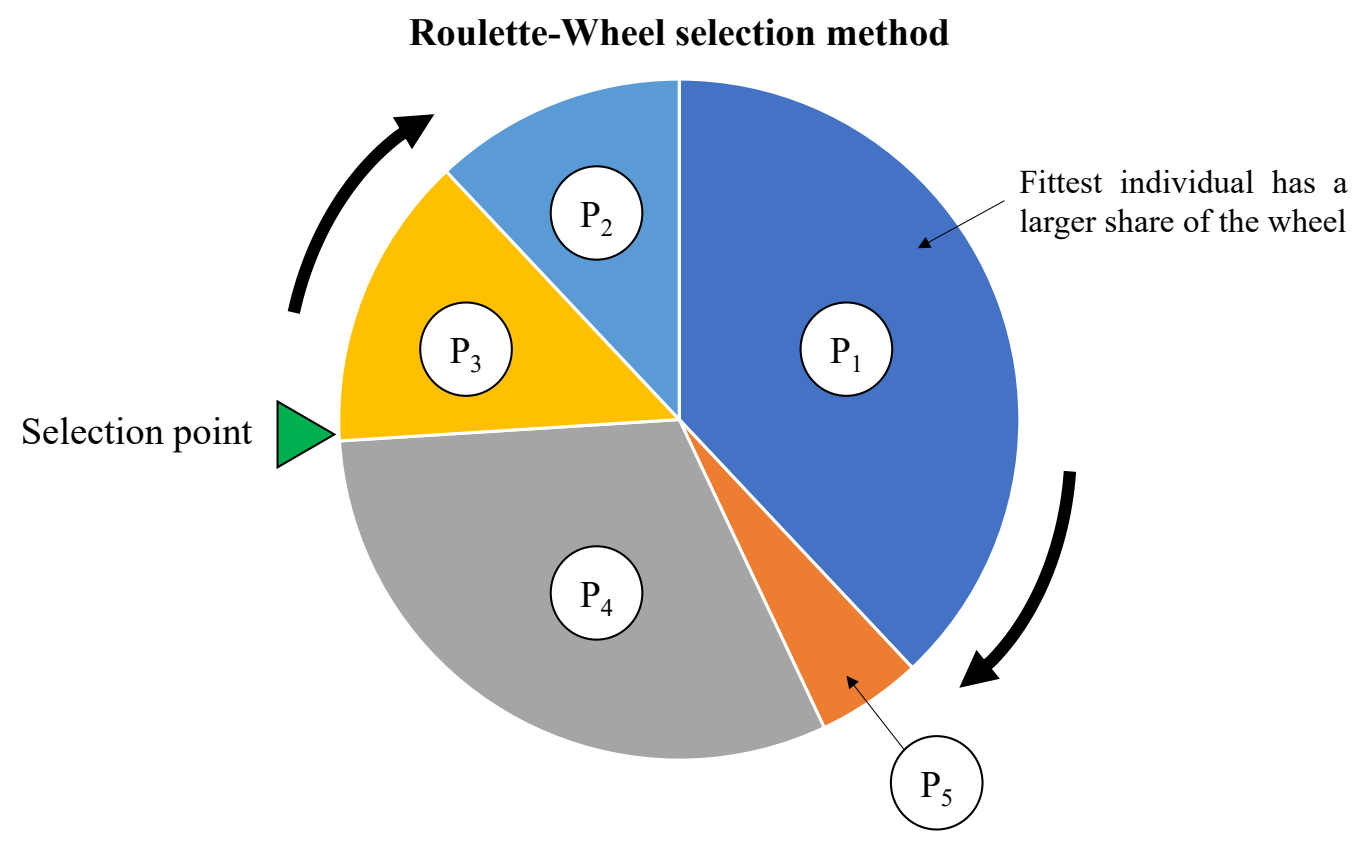

Figure 4.4: Roulette-Wheel selection method diagram with the probability of selection of each individual

The probability $P_{i}$ of an individual being selected, with respect to its fitness value $f(i)$, is given by the following relation,

$$
P_{i}=\frac{f(i)}{\sum_{k=1}^{N} f(k)}
$$

Hence, individuals with higher values of fitness have a higher probability of being selected. This fact, however, does not exclude the possibility of selecting individuals with lower fitness. The resultant effect is a greater genetic diversity to the following generation of individuals, decreasing the chances of trapping the solution in a maximum or minimum solution. The individual is selected 
based on the accumulated probability $q_{i}$, given by Equation (4.6), and compared with a randomly generated probability, $r$, between 0 and 1 . If $q_{i}>r$, the individual is selected for the further generations.

$$
q_{i}=\sum_{k=1}^{i} P_{k}, \quad k=1, \ldots, i
$$

The Algorithm 4.1 presents the Roulette-Wheel method.

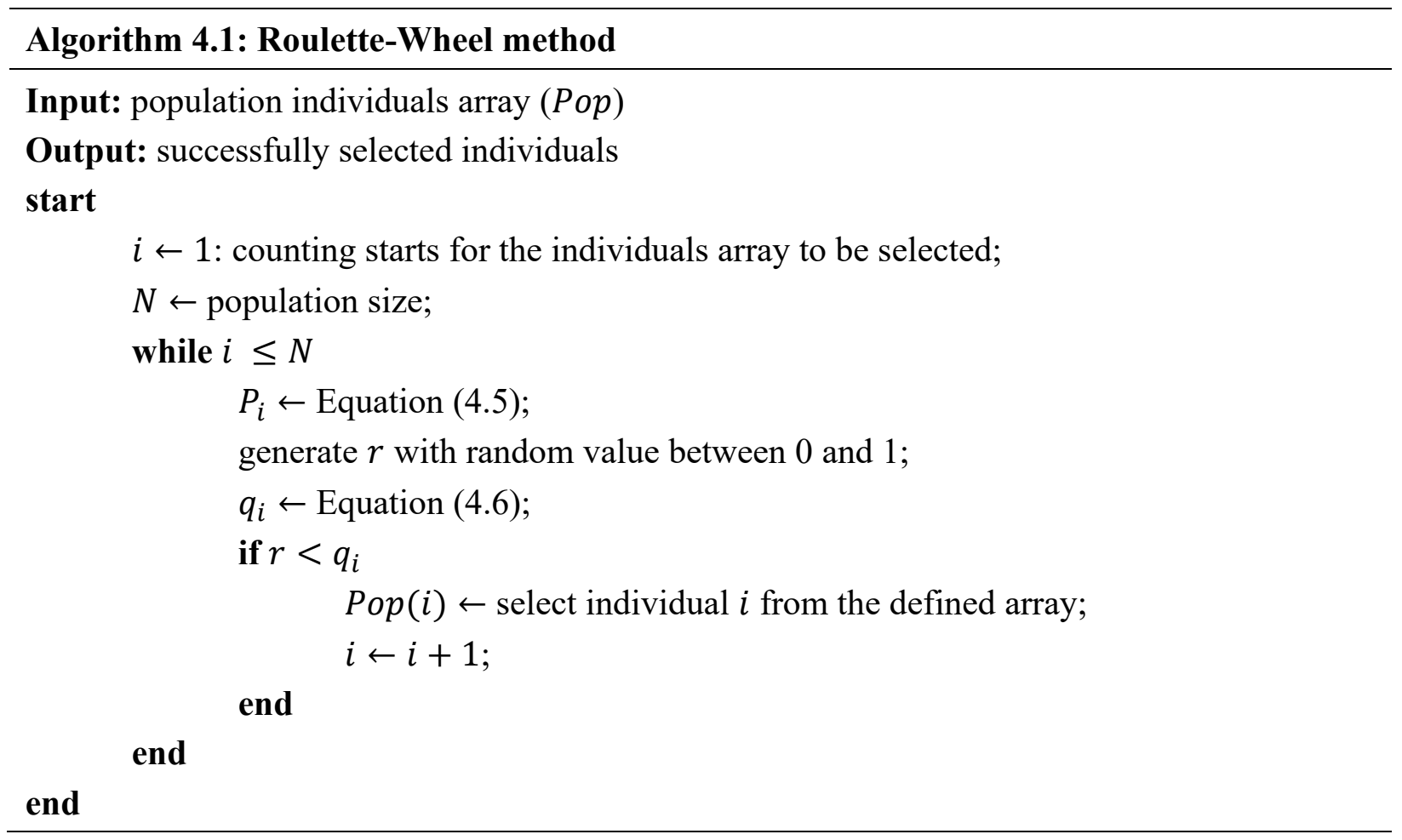

\subsubsection{Rank selection}

The linear rank selection was first introduced by Baker [114], and essentially avoids the fast convergence of the GA due to the introduction of a score system for each individual, therefore not only ranking the components with the fitness function. In this method, each individual in the 
population is ranked in ascending order of fitness evaluation. The one with lowest value receives a score of 1 , and the best receives a score $N$. The rest of the selection then follows the same process of the Roulette-Wheel method. The advantage of this method lies in the fact that the ranking criteria avoids giving most descendants a small group of individuals with high aptitude, which favors the characteristic of genetic diversity.

\subsubsection{Tournament selection}

The tournament selection is an interesting method where a portion of the current population is randomly chosen for analysis. The elements of this portion are compared between each other by means of their fitness values, and the one with highest score is selected.

The size of the tournament portion is defined by a parameter $p$, which sets the number of individuals to be compared. Figure 4.5 illustrates the concept of the tournament selection. It is easy to see that the minimal value for $p$ is 2 , such that a competition is able to be established. If $p$ is the same size $N$ of the population, the selected individual is always the same. For high values of $p$, the $N-p$ chromosomes will tend to predominate due to the highest probability of being chosen.

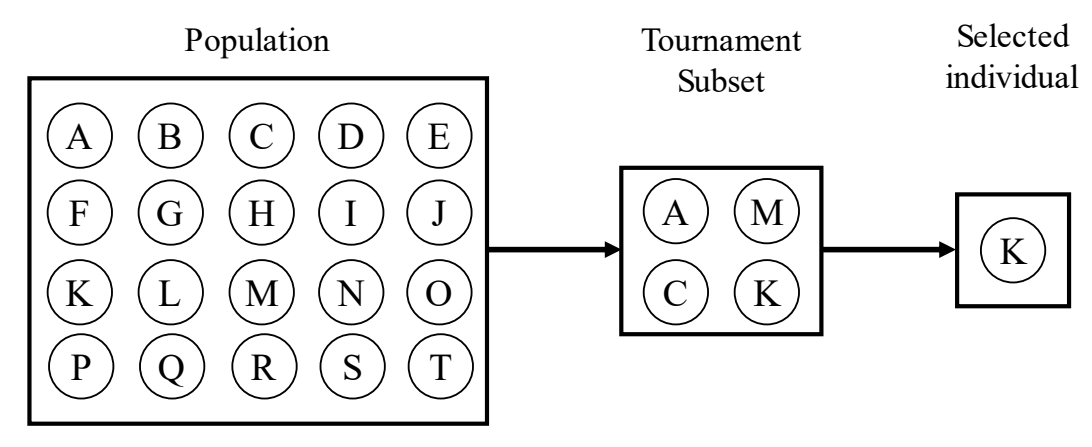

Figure 4.5: Tournament selection schematic

The Algorithm 4.2 presents the Tournament selection method. 


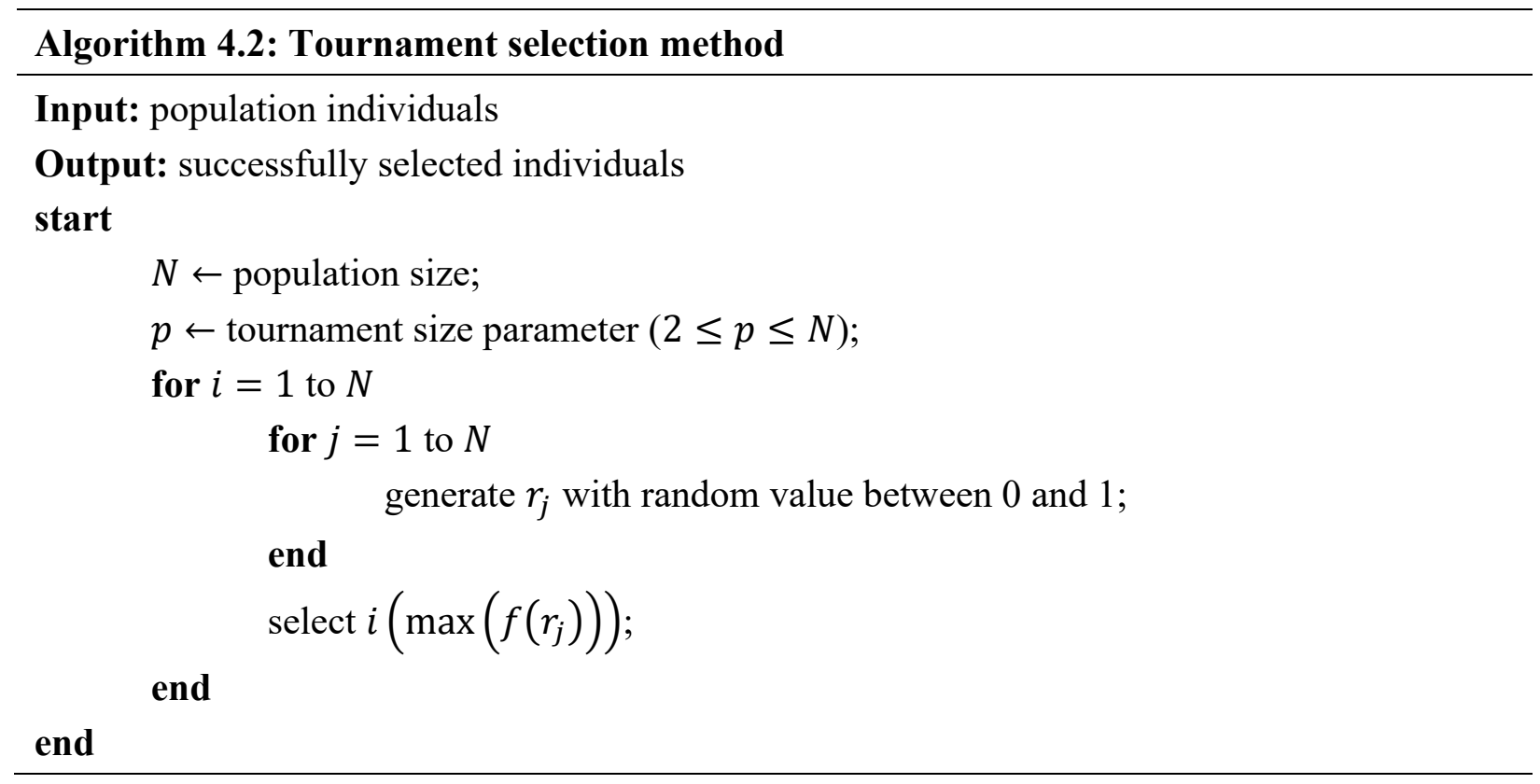

\subsubsection{Elitism and decimation}

The elitism is a deterministic method that preserves a small portion of individuals with the higher fitness values and, therefore, are not subjected to any type of operation to proceed in the further generations. It is important to emphasize that due to the deterministic nature, this parameter must assume a low value such that the genetic diversity is not affected or mitigated within new generations.

Similarly to the previous concept, the decimation is also a deterministic parameter, but now responsible to remove a fixed number of individuals with lower values of aptitude. Care must be taken when choosing the value of this parameter due to the fact that some potential feasible genetic characteristics of individuals may be eliminated in the decimation process. Usually, the elimination only takes place after a certain number of generations computed.

\subsubsection{Crossover}


Crossover is the mechanism responsible for the permutation of genetic material between parent individuals, in order to generate new offspring from the precedent population. According to Goldberg [109], intending to achieve efficiency in the crossover, a minimum value of probability of $P_{c}=60 \%$ must be assumed. Figure 4.6 depicts the single point crossover process between two parent chromosomes with binary codification.

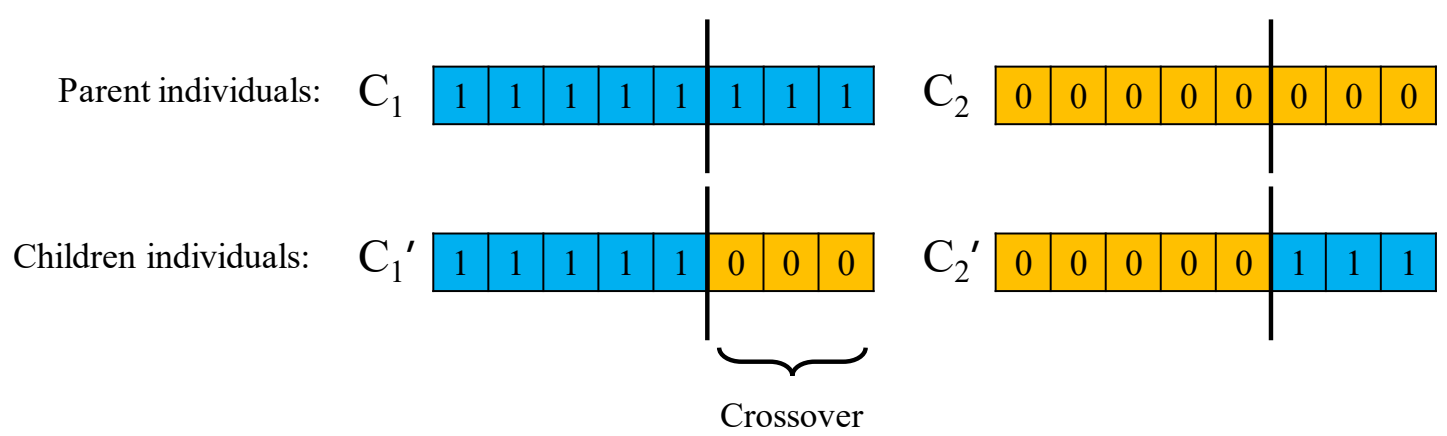

Figure 4.6: Single point crossover with binary codification

There are multiple ways to perform the crossover operation between chromosomes, and it is important to understand the difference between them. In addition to the single point method, it is also possible to carry out the procedure with two point random or fully random methods, as depicted in Figure 4.7. The GA procedure implemented in MATLAB ${ }^{\circledR}$ utilizes by default the fully random crossover, however it also allows the user to choose between different crossover methods. 


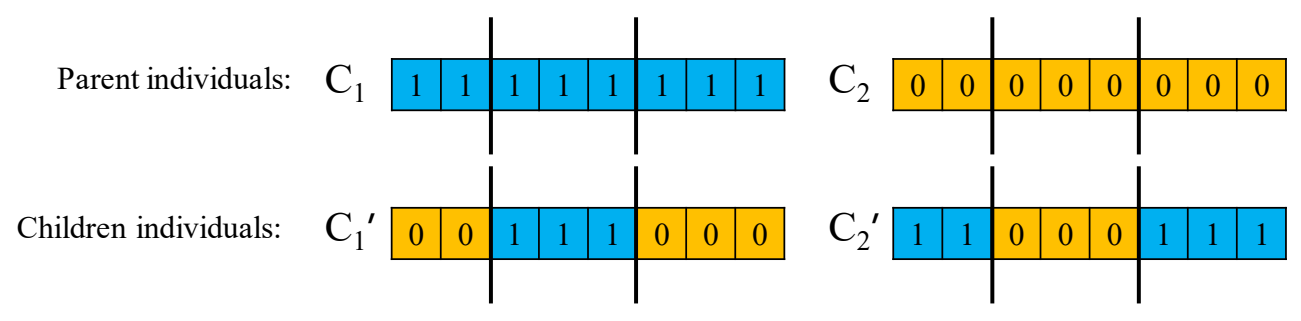

(a)

\begin{tabular}{|c|c|c|c|c|c|c|c|c|c|c|c|c|}
\hline \multirow[t]{2}{*}{ Parent individuals: } & 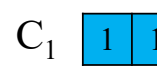 & \begin{tabular}{|l|l|}
1 & 1 \\
\end{tabular} & 1 & \begin{tabular}{l|l}
1 & 1 \\
\end{tabular} & \begin{tabular}{|l|l|}
1 & 1 \\
\end{tabular} & $\mathrm{C}_{2} 0$ & 0 & 0 & \begin{tabular}{c|c}
0 & 1 \\
\end{tabular} & & & 0 \\
\hline & & $\rrbracket$ & $\sqrt{2}$ & & $\sqrt{2}$ & & th & & $\sqrt{ }$ & & & $\sqrt{2}$ \\
\hline Children individuals: & $\mathrm{C}_{1}^{\prime} \quad 1$ & \begin{tabular}{|l|l|}
0 & 1 \\
\end{tabular} & 0 & \begin{tabular}{l|l}
1 & 1 \\
\end{tabular} & \begin{tabular}{|l|l|}
0 & 0 \\
\end{tabular} & $\mathrm{C}_{2}^{\prime} 0$ & 1 & 0 & & & & 1 \\
\hline
\end{tabular}

Figure 4.7: (a) Two point random and (b) Fully random crossover. The arrows indicate the respective allele that will go through the crossover operation

Other existent crossover procedures that are pertinent in the literature are the arithmetic, heuristic, Rayleigh, or the Blend crossover. An in-depth study of the difference between those methods is out of scope from this work, and therefore will not be discussed. If the reader has interest in the topic, the works of the following authors are recommended: Mitchell [108], Goldberg [109], Michalewicz and Schmidt [115], Deep and Thakur [116].

\subsubsection{Mutation}

The genetic operator of mutation is responsible for stimulating or introducing genetic variability in the new population. It is a probabilistic procedure that modifies one or more alleles of a gene, when a probability $P_{m}$ of the phenomena occurrence is introduced. In binary codification, it is easy to understand the implementation of this genetic operator, such that the inverse of a specific allele is imposed within the chromosome, as described in Figure 4.8. 

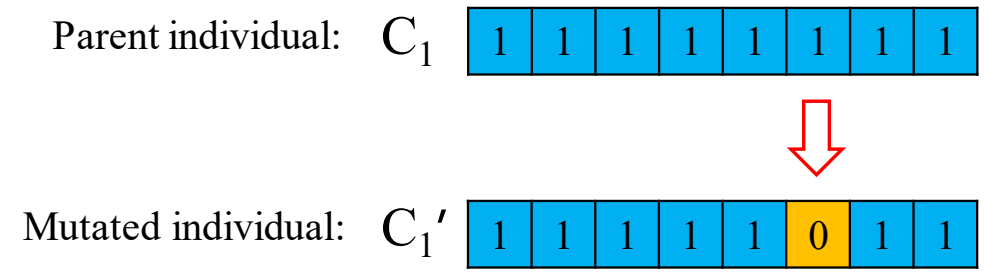

\section{Figure 4.8: Binary codification mutation operation}

With the inherent randomness characteristic of this operator, it is possible to introduce a change that would positively or negatively impact the performance of an individual. Therefore, the literature suggests the use of small probabilities values when defining this parameter in GA simulations. Goldberg [109] states that the use of $P_{m}=5 \%$ or less is sufficient.

Likewise, there are a multitude of algorithms to perform the mutation of individuals. Within the common methods, the highlights are the Uniform, Boundary, Gaussian, Uniform-creep, Nonuniform-creep, and adaptive mutation. The GA framework implemented in MATLAB ${ }^{\circledR}$ utilizes by default, for a simulation with constraints, the adaptive mutation [117]. In this method, the chromosomes are ranked with respect to their objective functions, and separated into two categories; being the low and high quality, according to the value of average population fitness. Individuals classified with low quality receive a higher mutation rate, while the ones with high quality are assigned with a lower mutation rate. More details about the adaptive mutation are discussed in the work from Libelli and Alba [118]. 


\section{Chapter 5: Theoretical framework part III - Implementation of a multi- objective design optimization procedure}

\subsection{Introduction}

In this chapter, a multi-objective optimization framework is proposed that determines the best values for the TMD set attached to the structural nodes of a given aerospace structure, intending to concurrently minimize the aeroelastic response and the total mass inclusion due to the placement of the control devices. The implemented framework is discussed in detail, where the computation of the MOGA procedure is presented. Then, the FE modelling of the TMD control devices is explored, with respect to the elements utilized and actual use of the programming cards utilized to achieve the configuration. Next, the entire algorithm is discussed, from the input parameters and calculation of the fitness function to the computation of the solution with the Utopian Point Method. Finally, the last section presents a validation study by employing the framework implemented.

\subsection{Optimization problem formulation}

The optimization problem is formulated as the following:

Fitness function: $\quad$ Minimize $\sum_{i=1}^{n_{T M D}} \mathbf{m}_{T M D}(i)$

Minimize $U_{\text {fitness }}$

Design variables: $\boldsymbol{\xi}=\left\{\mathbf{m}_{T M D}, \mathbf{c}_{T M D}, \mathbf{k}_{T M D}\right\}$

$$
\begin{array}{cl}
\text { Constraints: } & 0 \leq \sum_{i=1}^{n_{T M D}} \mathbf{m}_{T M D}(i) \leq(\alpha \times M) \\
& c_{T M D}^{\min } \leq \mathbf{c}_{T M D} \leq c_{T M D}^{\max }
\end{array}
$$




$$
k_{T M D}^{\min } \leq \mathbf{k}_{T M D} \leq k_{T M D}^{\max }
$$

where $\boldsymbol{\xi}=\left\{\mathbf{m}_{T M D}, \mathbf{c}_{T M D}, \mathbf{k}_{T M D}\right\}$ is the vector that contains values for mass, damping and stiffness of each TMD considered, $n_{T M D}$ is the total number of TMD that were attached to structural grid points, $U_{\text {fitness }}$ is a generic net output response of the system, computed with the root sum of squares (RSSQ) of the DOF. The position of each TMD could be similarly implemented using the proposed optimization framework, however it was considered to be out of scope of this work. In the case of a frequency domain analysis, the output response of the DOF is calculated using the TCLD formulation, for a determined critical frequency on the frequency response function. $M$ is the total mass of the structure analyzed, and $\alpha$ is a percentage value, defined by the user, to limit the TMD mass inclusion. It is important to mention that intending to simplify the variability of the masses of the TMD, all were considered the same, and the total inclusion should be less or equal than a chosen percentage of the uncontrolled structure's mass. The variables $c_{T M D}^{\min }, c_{T M D}^{\max }, k_{T M D}^{\min }$ and $k_{T M D}^{\max }$ represent the minimum and maximum bounds of the design space of the damping and stiffness parameters from the TMD set analyzed.

\subsection{Framework structure}

This section presents the in-depth understanding of the framework implemented. Initially, the multi-objective GA (MOGA) procedure is described, such that the population creation and other genetic operators, and the selection of best individuals from the Pareto front are discussed. Next, the FE modelling of the TMD is shown, indicating the element selection and how they are attached to the target structure. In the final part of this section, the algorithms implemented are presented and discussed. 


\subsubsection{Elitist multi-objective genetic algorithm}

The optimization procedure is carried out by means of an elitist Multi-Objective Genetic Algorithm (MOGA) [119-121] that computes the multiple-TMD optimal parameters for the structural model, which is subjected to the atmospheric wind disturbances as modeled in the previous sections.

In general lines, the MOGA begins by creating an initial population, with a predefined size, that satisfies the linear constraints and bounds imposed to the optimization problem. Then, parent individuals from the current population are selected by means of a binary tournament, and the children are created by crossover and mutation from the selection tournament. The children individuals are evaluated by using the fitness function outputs and feasibility, creating a score for each individual. The obtained children individuals are then combined, in matrix form, with the current population evaluated, generating the extended population. Within this new set, the rank and crowding distance are calculated for all individuals.

The rank is essentially a parameter that measures the chance of selection, and is related to the dominance by other individuals. When an individual is not dominated by others, i.e., classified as independent, it returns lower rank values, which represents the highest chances of feasibility and selection. The crowding distance is a measurement of closeness of individuals of the same rank to its nearest surrounding neighbors. Higher crowding distances of individuals under the same rank have a higher chance of selection. Finally, the last step of the MOGA is to trim the extended population generated to the actual predefined population size, by keeping a certain number of individuals from similar rank values. Moreover, this step is also responsible for applying the migration of individuals between iterations (using for example the migration interval input 
variable), which defines the set of best individuals from one subpopulation to replace the worst individuals in another subpopulation, in order to assist in the search for the optimal value of the fitness function.

\subsubsection{Tuned mass damper FE modelling}

Regarding the FE modelling of each TMD attached to a target structure, it can be done through a variety of ways. In MSC NASTRAN ${ }^{\circledR}$ environment, the modelling performed consists in concurrently using three different elements, being the CELAS2, which defines a scalar spring element, the CDAMP2, which defines a scalar viscous damp element, and the CMASS2, which defines a scalar concentrated mass element [122].

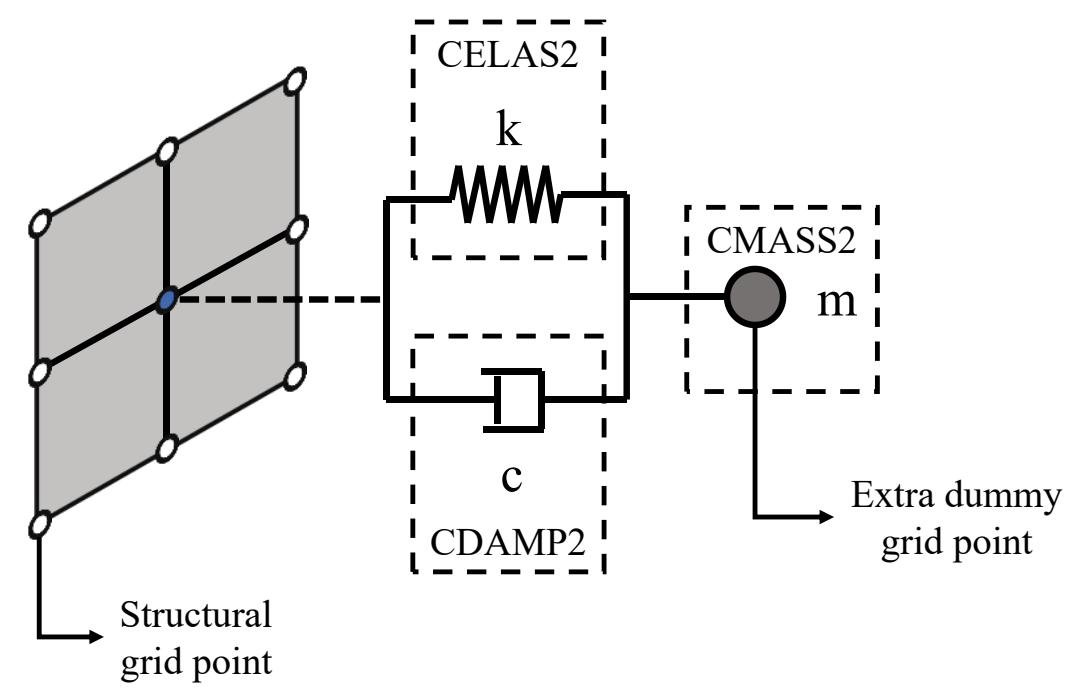

Figure 5.1: Schematics of the FE model of a translational TMD

The referred elements support multiple DOF of actuation, resulting in a multiple DOF TMD. In order to attach the control devices in a structural grid node, it is necessary to create an extra dummy node, that is used to place the CMASS2 element and interconnect the CELAS2 and CDAMP2 elements, as depicted in Figure 5.1. With this modelling it is possible to insert multiple TMD in a 
structure and evaluate the effect of changing the mass, stiffness and damping of each element that potentially minimizes the amplitude of response under dynamic excitations, by manipulating the EOM given by Equation (3.1).

In terms of input cards, each resonator is defined as depicted in Figure 5.2. As it will be shown in the following sections, it is possible to create a separate.$t x t$ file that contains the information of the TMD, and include it in the main solution file of the simulation. It is important to mention that the CMASS2 card only needs the input coordinates of the extra dummy grid point created, "G2", therefore allowing the effective actuation of the TMD. Moreover, it is easy to see how the connection between the TMD and the target structure is performed, i.e., by means of the definition of "G1" as the structural grid point, and its respective "C1" DOF of actuation.

\begin{tabular}{|l|l|l|l|l|l|l|}
\hline CELAS2 & EID & $\mathrm{K}$ & $\mathrm{G} 1$ & $\mathrm{C} 1$ & $\mathrm{G} 2$ & $\mathrm{C} 2$ \\
\hline CDAMP2 & EID & $\mathrm{B}$ & $\mathrm{G} 1$ & $\mathrm{C} 1$ & $\mathrm{G} 2$ & $\mathrm{C} 2$ \\
\hline CMASS2 & EID & $\mathrm{M}$ & & & $\mathrm{G} 2$ & $\mathrm{C} 2$ \\
\hline
\end{tabular}

\begin{tabular}{|l|l|}
\hline Parameter & Meaning \\
\hline EID & Element ID \\
\hline K & Stiffness of the scalar spring \\
\hline B & Damping coefficient \\
\hline M & Mass property \\
\hline G1, G2 & Geometric grid point ID \\
\hline C1, C2 & DOF of actuation (1 to 6) \\
\hline
\end{tabular}

Figure 5.2: MSC NASTRAN® ${ }^{\circledR}$ cards specification for the creation of TMD

\subsubsection{Algorithms implemented description}

Figure 5.3 depicts in detail the steps of the optimization procedure implemented. 

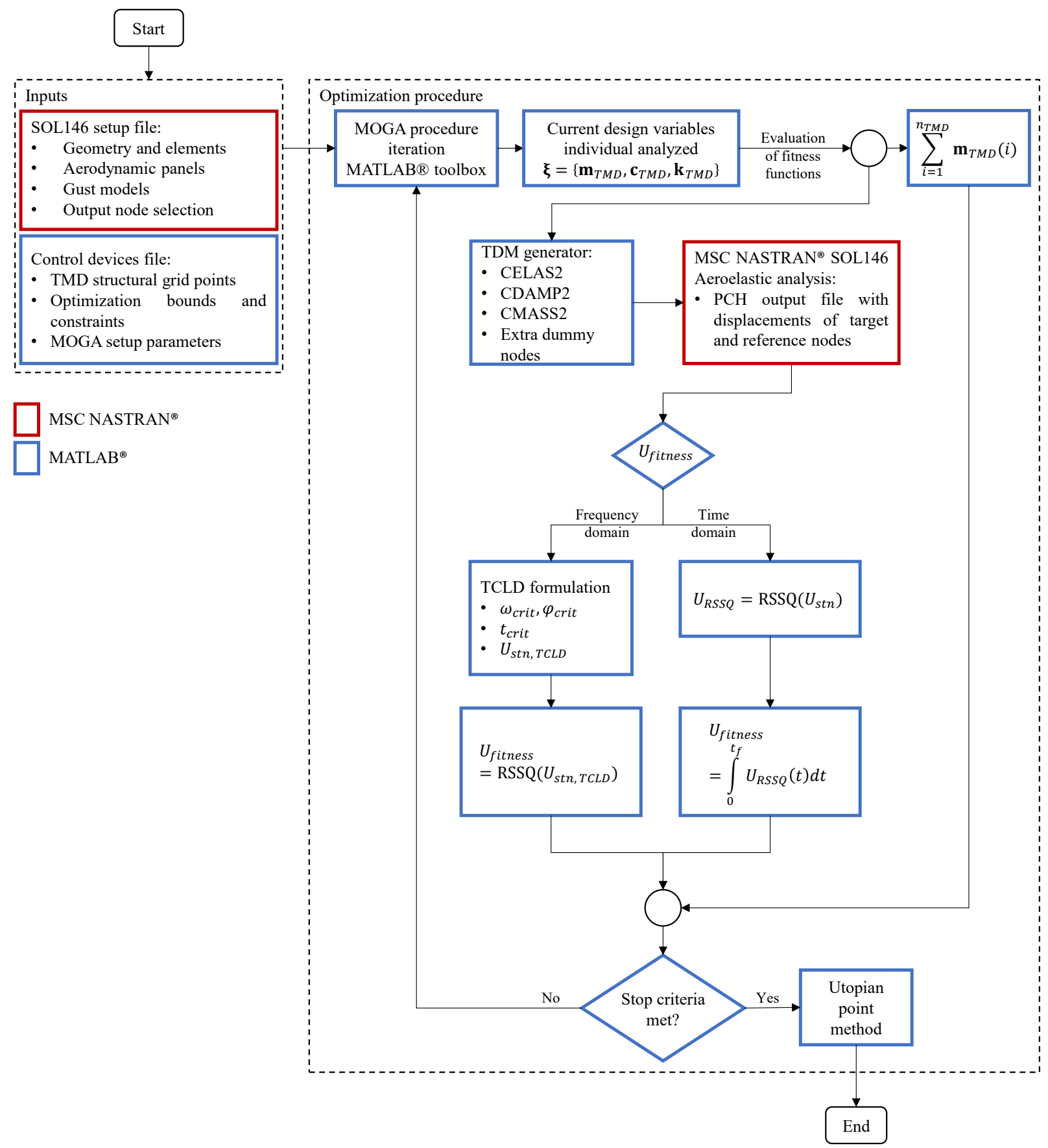

Figure 5.3: Flowchart of the optimization routine developed

Initially, the inputs are defined for the simulation setup file for the modelling of the dynamic aeroelastic analysis in MSC NASTRAN ${ }^{\circledR}$ (SOL146), which encompasses the geometry, element 
connection and properties of the structure, alongside with the aerodynamic DLM panels, and gust excitation inputs. The latter is computed in a separate script for given different initial conditions and atmospheric conditions. The TMD structural grid points of placement, the optimization bounds $\left(\mathbf{m}_{T M D}, \mathbf{c}_{T M D}, \mathbf{k}_{T M D}\right.$ values) and the GA setup parameters are also defined.

For some cases, it was also utilized a predefined initial population, which is essentially based in the Pareto front individuals from previous run, where the number of design variables is preserved. The input of optimal individuals for the initial population is beneficial for increasing the chances of finding a better solution with the same population size and generations analyzed [113].

In sequence, the iterative design optimization procedure begins by evaluating the fitness function considering the current individual, from the defined population. The total mass inclusion of the TMD is first calculated from the design variables inputs, followed by $U_{\text {fitness }}$. To determine the latter, a sequence of sub-steps is necessary:

- First, two files for input to MSC NASTRAN ${ }^{\circledR}$ are generated, being the extra dummy grid points created for the placement of the TMD according to the predefined location and $n_{T M D}$ used in the analysis, and the actual elements that compose the TMD given the values from the design variables. Those are represented by the CELAS2, CDAMP2 and CMASS2, which are the spring, viscous damper, and concentrated mass elements.

- Second, MSC NASTRAN® is started and the dynamic aeroelastic analysis computed with the given input files previously described.

- Third, the output response file is generated in the PCH format and loaded in the routine developed in MATLAB ${ }^{\circledR}$, and is defined in terms of displacement of the DOF from a selected node. 
- Fourth, if the frequency domain analysis is being performed, the displacement output response is adjusted with the TCLD formulation, where the peak response is determined for a critical frequency, and then $U_{\text {fitness }}$ is calculated by means of a RSSQ of the DOF. If the time domain analysis is being performed, $U_{R S S Q}$ is calculated directly with the RSSQ of the displacement DOF considered, and integrated over the time length of the signal analyzed, such that the total area under the $U_{R S S Q} \times t$ curve is minimized, therefore defining the value of $U_{\text {fitness }}$ for this case. The optimization procedure continues the iterative process until a stopping criterion is reached, such as the chosen maximum number of generations, or the average change in the spread of the Pareto front.

\subsubsection{The Utopian Point Method}

The multi-objective optimization method determines several sets of feasible optimal solutions according to the constraints defined, however, only a smaller set of solutions can be implemented at a time for a structure. Therefore, considering all optimal solutions, the best of them can be selected through a variety of ways. In this work, to quantitatively determine the best design variables set among the Pareto front points, the utopian point method was used [123] since it is an efficient candidate for a large set of solutions. 


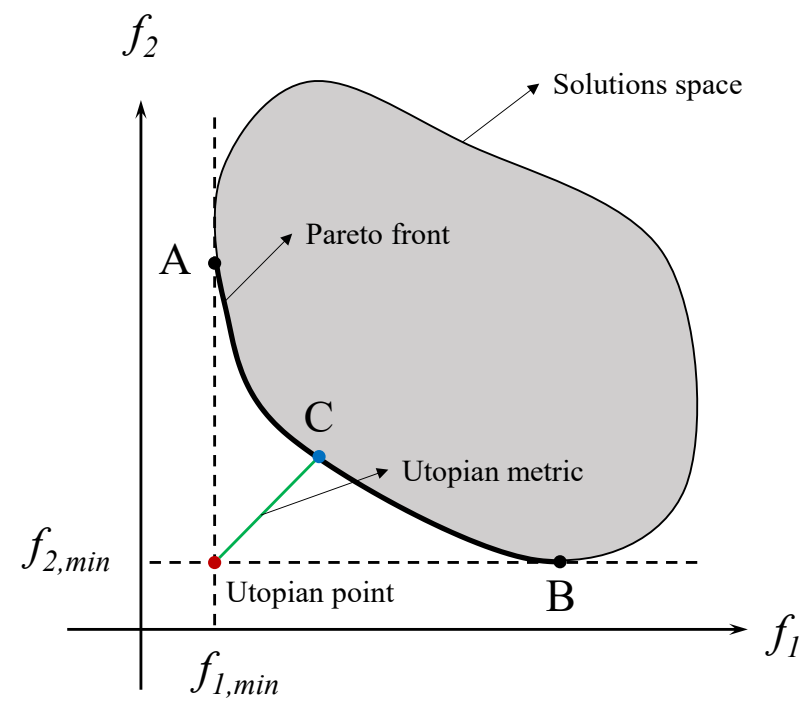

Figure 5.4: Utopian point of a Pareto front. The points A and B are the extremity individuals of the Pareto front, $C$ represents a point identified by the utopian metric utilized

The utopian point can be found by means of the coordinates of the extremities of the Pareto front, thus defined by values that simultaneously minimize both objective functions, as depicted in Figure 5.4. The standard use of the method employs the distance from the utopian point to the Pareto measured by means of the weighted Euclidean metric method, and the minimum distance overall defines the optimal set of design variables, representing the best trade-off between the objectives of the problem. It is interesting, however, to extend this approach for other arrangement of degree dependence of the utopian metric. The described method can be formulated to measure the distance between an individual located at the pareto Front, $\mathbf{F}(\boldsymbol{\xi})$, and the ideal point, $\mathbf{F}^{0}$, as in Equation (5.1),

$$
d=\min _{\xi}\left\{\sum_{i=1}^{N}\left|w_{i}\left(f_{i}-f_{i}^{0}\right)\right|^{q}\right\}^{1 / q}, \quad q \in \mathbb{N}
$$


where $d=d(\xi)$ is the calculated minimum utopian metric between the utopian point, $\mathbf{F}^{0}=$ $\left(f_{1}^{0}, f_{2}^{0}, f_{3}^{0}, \ldots, f_{N}^{0}\right)=f_{i}^{0}$, and the Pareto front point, $\mathbf{F}(\xi)=\left(f_{1}, f_{2}, f_{3}, \cdots, f_{N}\right)=f_{i}$, with a total of $N$ objectives. The exponent $q$ essentially defines the degree of the utopian metric, and can vary from 1 to $+\infty$, reflecting different penalty weighting for points that are located further away from the ideal point. For the value of $q=1$, the metric is defined by a linear weighted average of distances from the Pareto front individuals and the utopian point. For the referred value, only points that are located at the convex prominence of the front are selected. In the case of the value of $q=$ 2 , the metric is defined by the standard Euclidean distance. With the increase of $q$, more points of the Pareto front are selected as feasible individuals, considering different weight arrangements defined, until a maximum of $q=+\infty$, where all individuals are selected [39]. For this work, the standard Euclidean distance was utilized as the metric for the utopian point method.

The weights $w_{i}, i \in\{1, \cdots, N\}$ are defined as individual contributions originated from the objectives, representing a criterion of importance defined by the user [123]. For different definitions of the weight contribution, different individuals can be selected from the Pareto front. This fact is a consequence of the invariant characteristic of the Pareto front to monotonic transformations, therefore allowing one to determine the optimum individuals from the front and then investigating distinct metric schemes and weighting values without additional computation. An interesting in-depth discussion about the effects of metric weight selection is provided in the works of Lu and Anderson-Cook [123], Kasprzak and Lewis [39], and Szparaga et al. [124]. For simplicity of solution, in the case studies of this thesis to be presented in the further chapters, the weights are set as unitary values, such that the trade-off between objectives is equally balanced. 
The Algorithm 5.1 presents the procedure to determine the best individual of a given Pareto front based on the utopian point method with Euclidean metric and weight contribution for each objective function.

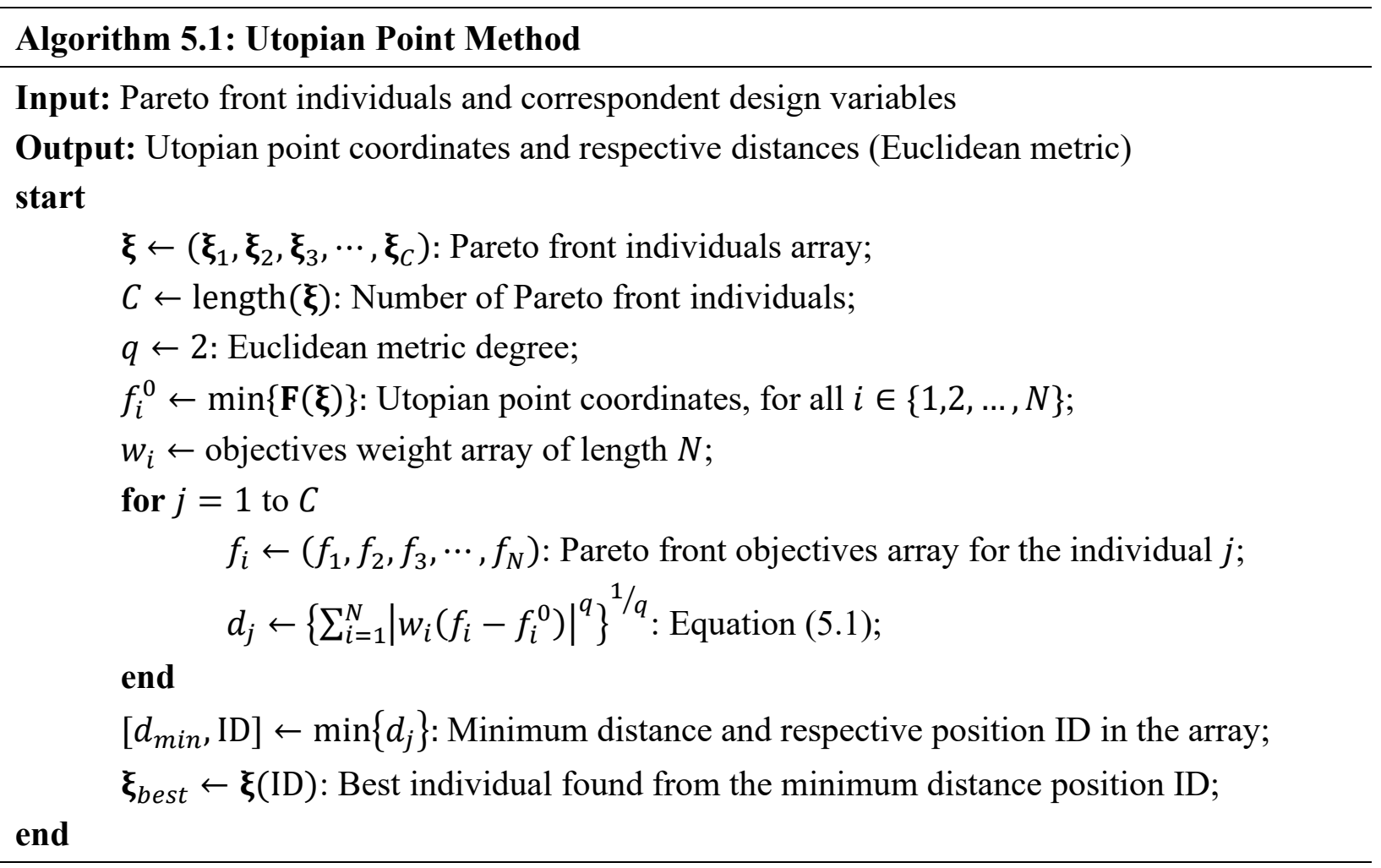

\subsection{Simplified framework validation study}

It is pertinent to validate the optimization framework developed, in order to guarantee that it is possible to determine the optimal parameters of TMD systems. Moreover, it is important to mention that the framework to be presented is a simplified version of the one presented in this Chapter, however, still possessing the same algorithm and calculation procedure, hence making it a correct representation.

To test the efficiency of the method, a simple case study of a 1-DOF mass-spring is investigated, where the main structure is undamped and the attached TMD assumes a viscous damping element. 
The optimal constitutive parameters of the TMD are found by utilizing the GA framework implemented herein, and compared with the analytical solution found in the literature. Furthermore, the MATLAB ${ }^{\circledR}$ scripts created are given in the Appendix A of the thesis.

\subsubsection{Analytical formulation of the problem}

Figure 5.5 depicts the schematics of the dynamic system studied, where $k_{s}$ and $k_{d}$ are the stiffness of the springs for the structural mass and the attached TMD, $m_{s}$ and $m_{d}$ are the mass of the structure and the attached TMD, $c_{d}$ is the viscous damping coefficient of the TMD, $p$ is a sinusoidal harmonic excitation, with frequency $\Omega$, applied to the structure with DOF defined as $u_{s}$ and $u_{d}$.

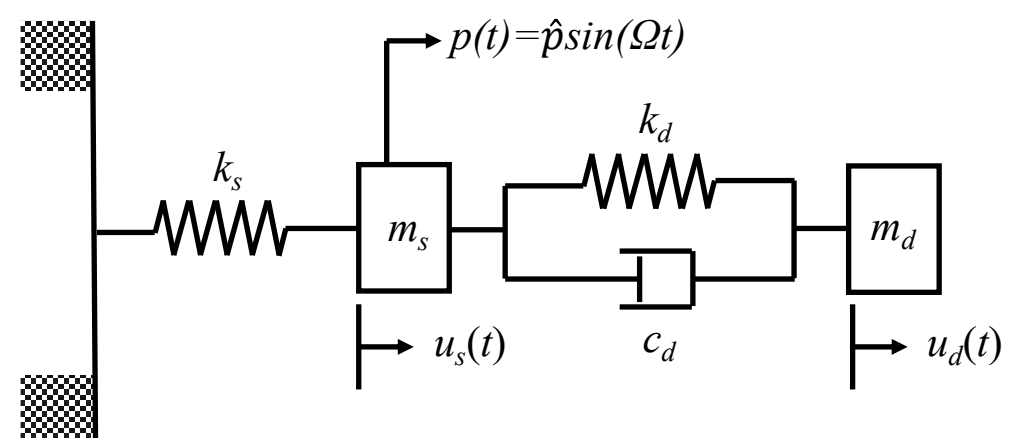

Figure 5.5: Undamped SDOF system coupled with a damped TMD device

The equations of motion of this system are given by:

$$
\left\{\begin{array}{c}
m_{d} \ddot{u}_{d}+c_{d} \dot{u}_{d}+k_{d} u_{d}+m_{d} \ddot{u}=0 \\
m_{s} \ddot{u}+k_{s} u_{s}-c_{d} \dot{u}_{d}-k_{d} u_{d}=p
\end{array}\right.
$$

Due to the simplicity of the problem, it is possible to obtain a closed analytical solution for the dynamic motion. The response for $u_{s}$ and $u_{d}$ are expressed as: 


$$
\left\{\begin{array}{l}
u_{s}=\hat{u}_{s} e^{i \Omega t} \\
u_{d}=\hat{u}_{d} e^{i \Omega t}
\end{array}\right.
$$

where $\hat{u}_{s}$ and $\hat{u}_{d}$ are the amplitude of displacement, and are taken complex quantities. Substituting Equations (5.3) in Equations (5.2) and solving the system, the response amplitude can be expressed in the polar form, given by:

$$
\left\{\begin{array}{l}
\hat{u}_{s}=\frac{\hat{p}}{k_{s}} H_{1} e^{i \delta_{1}} \\
\hat{u}_{d}=\frac{\hat{p}}{k_{s}} H_{3} e^{-i \delta_{2}}
\end{array}\right.
$$

where the $\delta$ variables are the phase angles between response and excitation, for the main structure and the TMD. The $H$ parameters are the amplification factors associated with the response. They are defined as the following:

$$
\begin{gathered}
H_{1}=\frac{\sqrt{\left(f^{2}-\rho^{2}\right)^{2}+\left(2 \xi_{d} \rho f\right)^{2}}}{\left|D_{2}\right|}, \quad H_{3}=\frac{\rho^{2}}{\left|D_{2}\right|} \\
\left|D_{2}\right|=\sqrt{\left(\left(1-\rho^{2}\right)\left(f^{2}-\rho^{2}\right)-\bar{m} \rho^{2} f^{2}\right)^{2}+\left(2 \xi_{d} \rho f\left[1-\rho^{2}(1+\bar{m})\right]\right)^{2}}
\end{gathered}
$$

The parameter $f=\omega_{d} / \omega$ is the ratio of natural frequency of the TMD and the structure, $\rho=\Omega / \omega$ is the ratio between the excitation frequency and the natural frequency of the structure, $\bar{m}=m_{d} / m$ is the mass ratio between the TMD and the structure, and finally $\xi_{d}$ is the damping ratio of the TMD device. As for the phase angles, they are formulated as: 


$$
\begin{gathered}
\delta_{1}=\alpha_{1}-\delta_{3} \\
\tan \alpha_{1}=\frac{2 \xi_{d} \rho f}{f^{2}-\rho^{2}}, \quad \tan \delta_{3}=\frac{2 \xi_{d} \rho f\left(1-\rho^{2}(1+\bar{m})\right)}{\left(1-\rho^{2}\right)\left(f^{2}-\rho^{2}\right)-\bar{m} \rho^{2} f^{2}}
\end{gathered}
$$

As presented in Connor [42], for a given mass ratio $\bar{m}$, the optimal parameters of the TMD that minimizes the amplitude of response of the structure modelled herein, $H_{1}$, are given by:

$$
\begin{gathered}
\left.f\right|_{\text {opt }}=\frac{\sqrt{1-0.5 \bar{m}}}{1+\bar{m}}, \quad\left(\left.\omega_{d}\right|_{\text {opt }}\right)^{2}=\left(\left.f\right|_{\text {opt }}\right) \omega \\
\left.\xi_{d}\right|_{\text {opt }}=\sqrt{\frac{\bar{m}(3-\sqrt{0.5 \bar{m}})}{8(1+\bar{m})(1-0.5 \bar{m})}} \\
\left.k_{d}\right|_{\text {opt }}=m_{d}\left(\left.\omega_{d}\right|_{\text {opt }}\right)^{2}=\bar{m} k_{s}\left(\left.f\right|_{o p t}\right)^{2} \\
\left.c_{d}\right|_{\text {opt }}=2\left(\left.\xi_{d}\right|_{\text {opt }}\right)\left(\left.\omega_{d}\right|_{\text {opt }}\right) m_{d}=\bar{m}\left(\left.f\right|_{\text {opt }}\right)\left[2 \omega m\left(\left.\xi_{d}\right|_{\text {opt }}\right)\right]
\end{gathered}
$$

\subsubsection{Optimization problem solution with GA}

The idea now is to estimate the optimal values for the TMD parameters, which can be found with the equations above, but using the implemented optimization framework based on GA. The optimization is carried out by defining the design variables as the pair $\left(f, \xi_{d}\right)$, with a constant input of the mass ratio $\bar{m}$ for each analysis. The amplitude of response of the structure, $H_{1}$, is takes as 
the fitness function of the optimization problem, and it is calculated with Equations (5.5.a) and (5.5.b).

The goal of the optimization is to determine the optimal design variables set that minimize $H_{1}$, for given values of $\bar{m} \in[0 \%, 10 \%]$. Ideally, the solution found from the GA should match the optimal analytical solution for every different $\bar{m}$ input, demonstrating that the framework implemented is capable of finding the best individual among a wide population range.

For each analysis, the population range is systematically recalculated based on the input value of $\bar{m}(i)$, and it is defined as the following:

$$
\begin{array}{ll}
\text { Frequency ratio: } & 0.01 \times\left. f\right|_{o p t}(i) \leq f \leq 100 \times\left. f\right|_{\text {opt }}(i) \\
& \\
\text { Damping ratio: } & 0.01 \times\left.\xi_{d}\right|_{\text {opt }}(i) \leq \xi_{d} \leq 100 \times\left.\xi_{d}\right|_{\text {opt }}(i)
\end{array}
$$

To solve the optimization problem, it is assumed a mass-spring structure with the mechanical properties set as $k_{s}=5000 \mathrm{~N} / \mathrm{m}$ and $m_{s}=5 \mathrm{~kg}$. The fastest convergence of the GA, for each iteration, is found when using the following parameters:

- Number of generations: 100;

- Population size: 100 ;

- Crossover probability: $80 \%$;

- Elitism probability: 5\%;

- Mutation probability: 2\%;

- Fitness function convergence tolerance: 1E-3. 

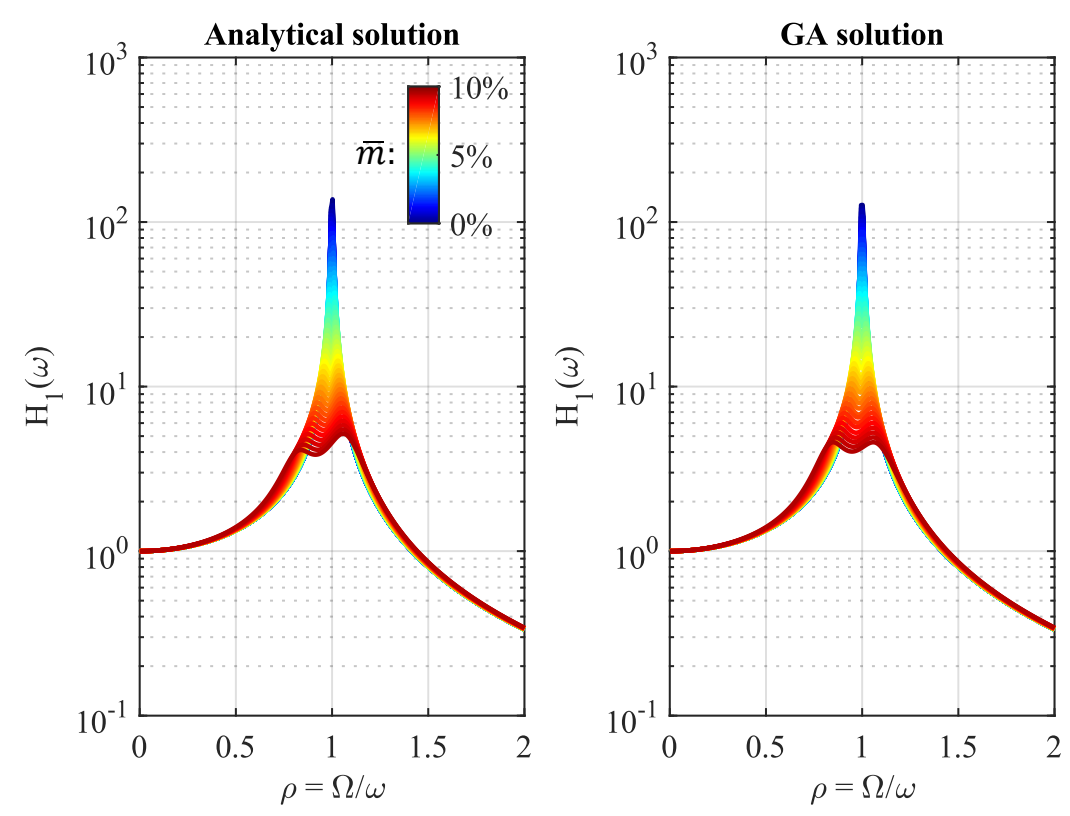

(a)
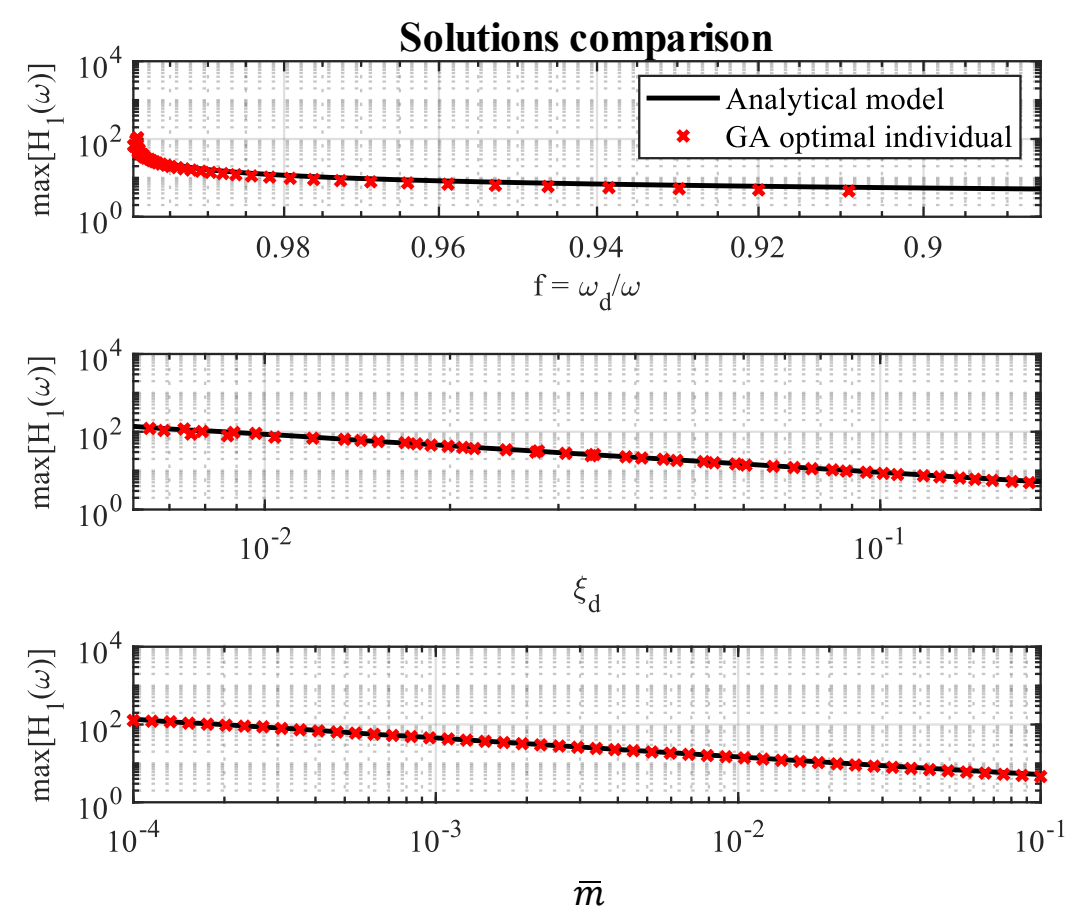

(b)

Figure 5.6: Results for the validation case study. (a) Amplitude of response $\mathrm{H} 1$ with respect to the excitation frequency ratio for the solutions obtained with the analytical model and the GA framework. (b) Comparison of the optimal individuals obtained with the GA 
This way, a maximum of 10,000 iterations are computed by the GA, per mass ratio $\bar{m}(i)$ analyzed. Figure 5.6 depicts the solutions obtained with the numerical experiment, compared with the analytical solutions obtained by using the Equations (5.7.a) to (5.7.d). It is possible to observe that the individuals obtained with the GA framework present excellent fit with the analytical solutions, demonstrating the effectiveness of the procedure implemented.

Furthermore, Table 5.1 presents the selection of some of the optimal individuals, comparing them with the respective analytical solution. The low percentage error obtained in the design variables also indicates that the framework is capable of finding the best solution that closely matches the optimal design parameters of TMD.

Table 5.1: Selection of optimal design variables comparing the analytical and GA solutions

\begin{tabular}{ccc}
\hline \multicolumn{3}{c}{$\bar{m}=0.17 \%$} \\
\hline Model/ Var. & $\left.f\right|_{\text {opt }}$ & $\left.\xi_{d}\right|_{\text {opt }}$ \\
\hline Analytical & 0.9979 & 0.0249 \\
GA & 0.9985 & 0.0247 \\
Error [\%] & 0.0561 & 1.0656 \\
\hline
\end{tabular}

\begin{tabular}{ccc}
\hline \multicolumn{3}{c}{$\bar{m}=5.69 \%$} \\
\hline Model/ Var. & $\left.f\right|_{\text {opt }}$ & $\left.\xi_{d}\right|_{o p t}$ \\
\hline Analytical & 0.9326 & 0.1400 \\
GA & 0.9462 & 0.1425 \\
Error [\%] & 1.4525 & 1.7805 \\
\hline
\end{tabular}

\begin{tabular}{ccc}
\hline \multicolumn{3}{c}{$\bar{m}=8.69 \%$} \\
\hline Model/Var. & $\left.f\right|_{\text {opt }}$ & $\left.\xi_{d}\right|_{\text {opt }}$ \\
\hline Analytical & 0.8999 & 0.1707 \\
GA & 0.9200 & 0.1747 \\
Error [\%] & 2.2324 & 2.3476 \\
\hline
\end{tabular}




\section{Chapter 6: Case study I - Aeroelastic response attenuation of a simplified Earth-based radio antenna model}

\subsection{Introduction}

The methodology presented in the previous chapters will be now applied to a prototype of a large Earth-based radio antenna structure that is subjected to atmospheric wind disturbances and turbulence. Those antennas are usually employed in VLBI applications, which is a technique commonly used in radio astronomy to represent high-resolution images of celestial objects originated from space radiation signals, such as galaxies, planets, nebulas, among others [125127]. The VLBI is essentially a geometrical technique, where a set of Earth-based antennas is used to simultaneously aim to an artificial or natural radio signal source, which passes through a postprocessing correlation due to the time delay between the signal arrival in the antennas, allowing the creation of radio images [128].

Hence, the pointing accuracy of the antennas is a parameter of pivotal importance to obtain highresolution signals. Due to the large dimensions of those aerospace structures, they are constantly subjected to atmospheric wind disturbances, therefore minimizing the aeroelastic response is of great interest to allow the minimization of the pointing error. The implementation of multipleTMD devices is an interesting approach for this application, since it is highly efficient in attenuating dynamic modal responses and also it is a passive vibration control technique. The performance of this approach has not yet been investigated in the literature, and thus will be presented in this thesis. 


\subsection{Finite element model of the Earth-based radio antenna structure}

The case study structure is essentially composed by a Kagome-like truss inner geometry with a hexagonal outer shape and four boom arms that support the secondary reflector with an offset to the primary reflector, as depicted in Figure 6.1. Each member of the truss has 25 in of length with a cross-section area of $1 \mathrm{in}^{2}$, manufactured with aluminum 6061-T6 [129] (modulus of elasticity of $10000 \mathrm{ksi}$ and density of $0.0975 \mathrm{lb} / \mathrm{in}^{3}$ ), resulting in a total estimated mass of approximately $1183 \mathrm{lb}$. The choice of constitutive element to represent the antenna structure is a beam element CBAR with two nodes and six DOF per node. The frame is supported by 12 nodes located in the backside truss of the primary reflector, as indicated in Figure 6.1.

In order to evaluate the pointing accuracy of the antenna, two nodes of importance need to be defined, being the reference and target node. The first is located at the center of the frontside truss, while the second is located at the junction of the four boom arms, also shown in Figure 6.1. The pointing accuracy is modelled as the deviation angle between the target and reference nodes using the Equations (6.1.a) to (6.1.d),

$$
\begin{aligned}
& \Theta=\tan ^{-1} \frac{\Delta x}{\Delta L} \\
& \Phi=\tan ^{-1} \frac{\Delta y}{\Delta L} \\
& \Psi=\tan ^{-1} \frac{\Delta z}{\Delta L}
\end{aligned}
$$




$$
U_{R S S}=\sqrt{\Theta^{2}+\Phi^{2}+\Psi^{2}}
$$

where $\Theta, \Phi$ and $\Psi$ are the calculated deviation angles respectively in the $x, y$ and $z$ axes. The variables $\Delta x, \Delta y$, and $\Delta z$ are the time consistent displacements originated from the dynamic aeroelastic response in the $x, y$ and $z$ directions, $\Delta L$ is the fixed offset distance between the target and reference nodes, and $U_{R S S Q}$ is the net deviation angle of the structure, thus defining the pointing accuracy.

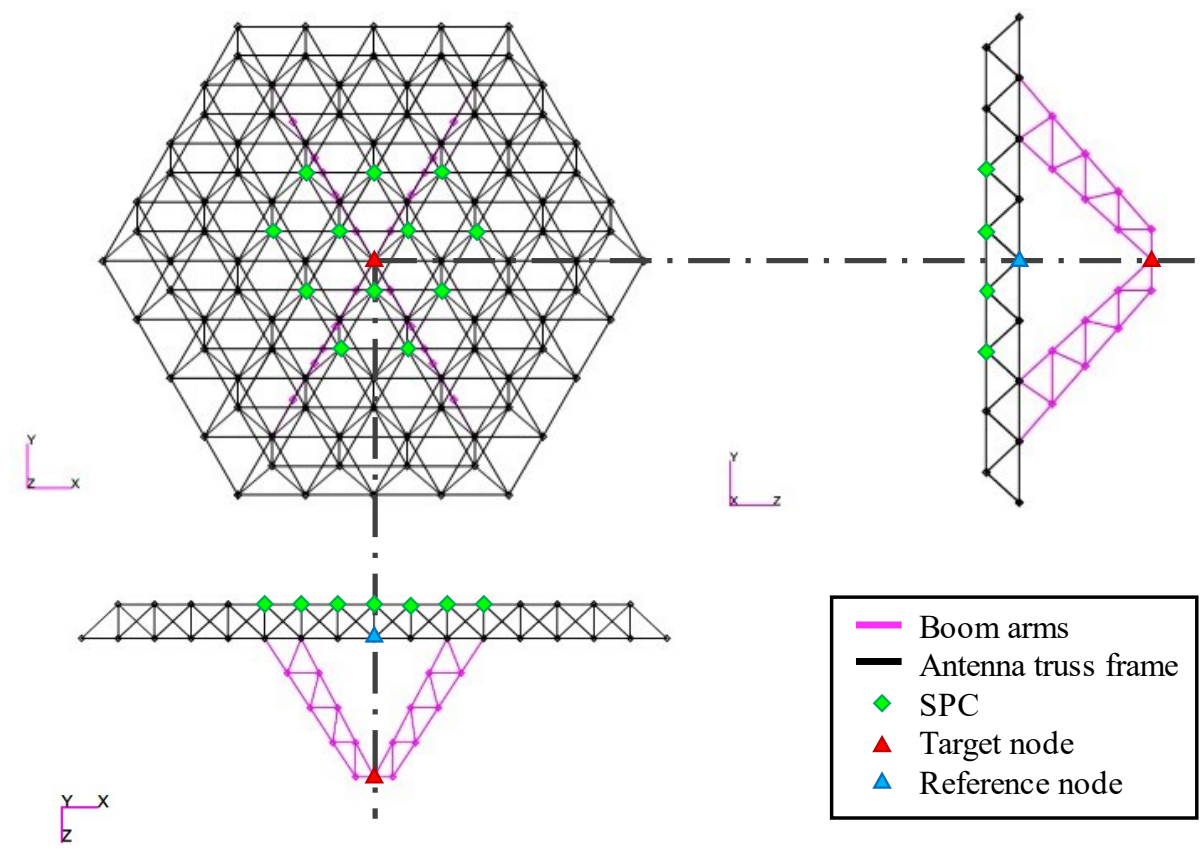

Figure 6.1: Schematics visualization of the FE model of the Earth-based radio antenna structure investigated

\subsection{Aeroelastic response of the uncontrolled model}

The primary reflector of the antenna frame is considered as the main aerodynamic surface that will result in relevant values of disturbance cause by the wind-gust excitations, modelled with the DLM 
panels. It is approximated as a flat surface, due to the low thickness to chordwise length ratio evaluated in approximately $6 \%$. The aerodynamic approximation is implemented with two main aeropanels containing 100 boxes each, where the wind-gust disturbance is defined as a vector perpendicular to the plane of the primary reflector surface, and the mean wind speed vector being parallel to it, as depicted in Figure 6.2.

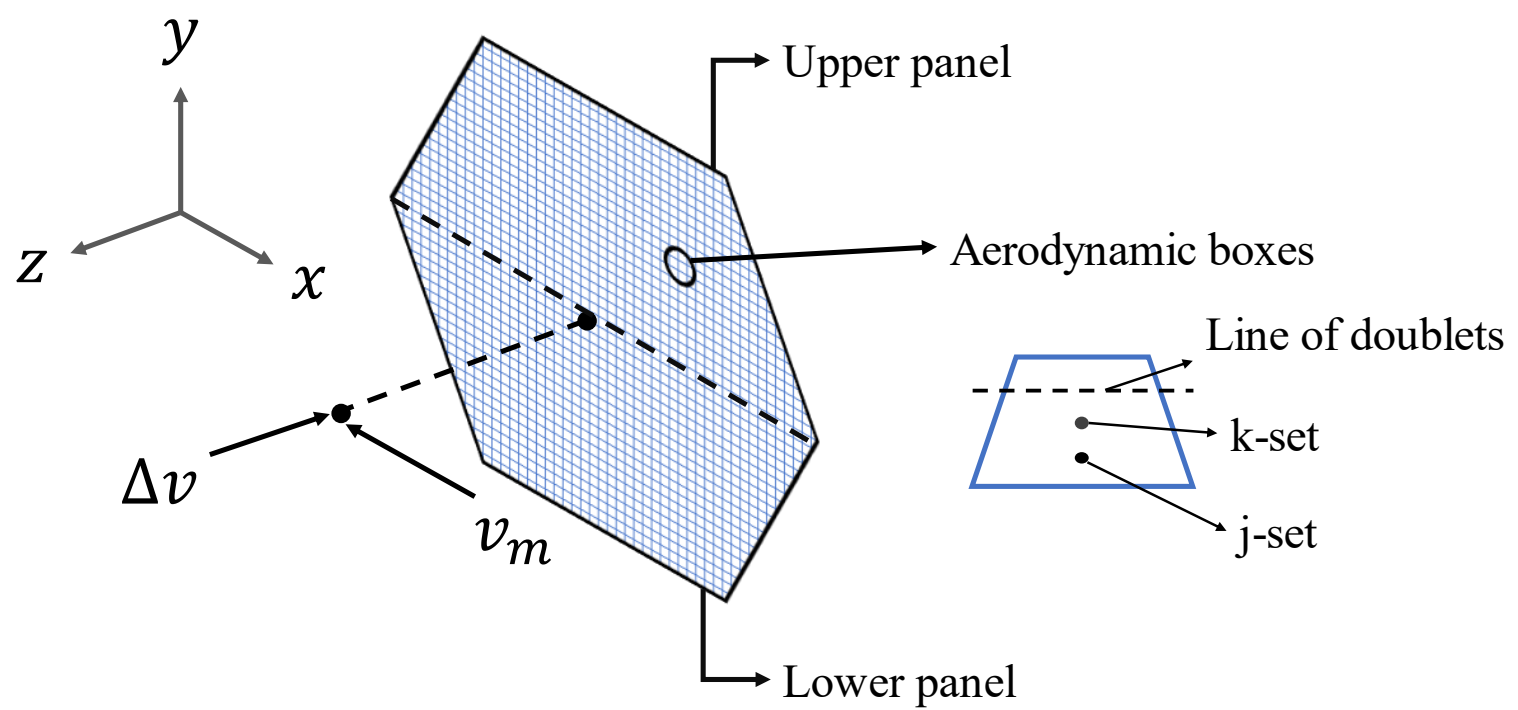

Figure 6.2: DLM panels modelling of the aerodynamic surfaces of the Earth-based antenna frame

Two operational cases were considered for the analysis of the proposed antenna model, in which the range of actuation was calculated for TDG and PSD formulations, respectively using the Equations (3.16.a), (3.16.b), and (3.17.a) to (3.17.c). The gust scale length utilized in the analysis was based on the values given by Nieto et al. [1], defined as $L_{g}=47,244.094$ in. In addition, the mean wind speed and the disturbance speed of the scenarios utilized are summarized in Table 6.1, and were given by the industrial partner of the project and the National Aeronautics and Space 
Administration (NASA) based on real operational conditions estimated at installation sites [38].

The wind-gust models considering the referred operational scenarios are depicted in Figure 6.3.

Table 6.1: Operational cases summary [38]

\begin{tabular}{ccccc}
\hline Case ID & $\begin{array}{c}\text { Disturbance speed } \\
\Delta v[\mathrm{in} / \mathrm{s}]\end{array}$ & $\begin{array}{c}\text { Mean wind speed } \\
v_{m}[\mathrm{in} / \mathrm{s}]\end{array}$ & Mach number & $\begin{array}{c}\text { Dynamic pressure } \\
{\left[\mathrm{lb} / \mathrm{in}^{3}\right]}\end{array}$ \\
\hline Primary & 109.10 & 349.95 & 0.02615 & 0.005686 \\
Secondary & 339.20 & 1093.61 & 0.08173 & 0.055524 \\
\hline
\end{tabular}
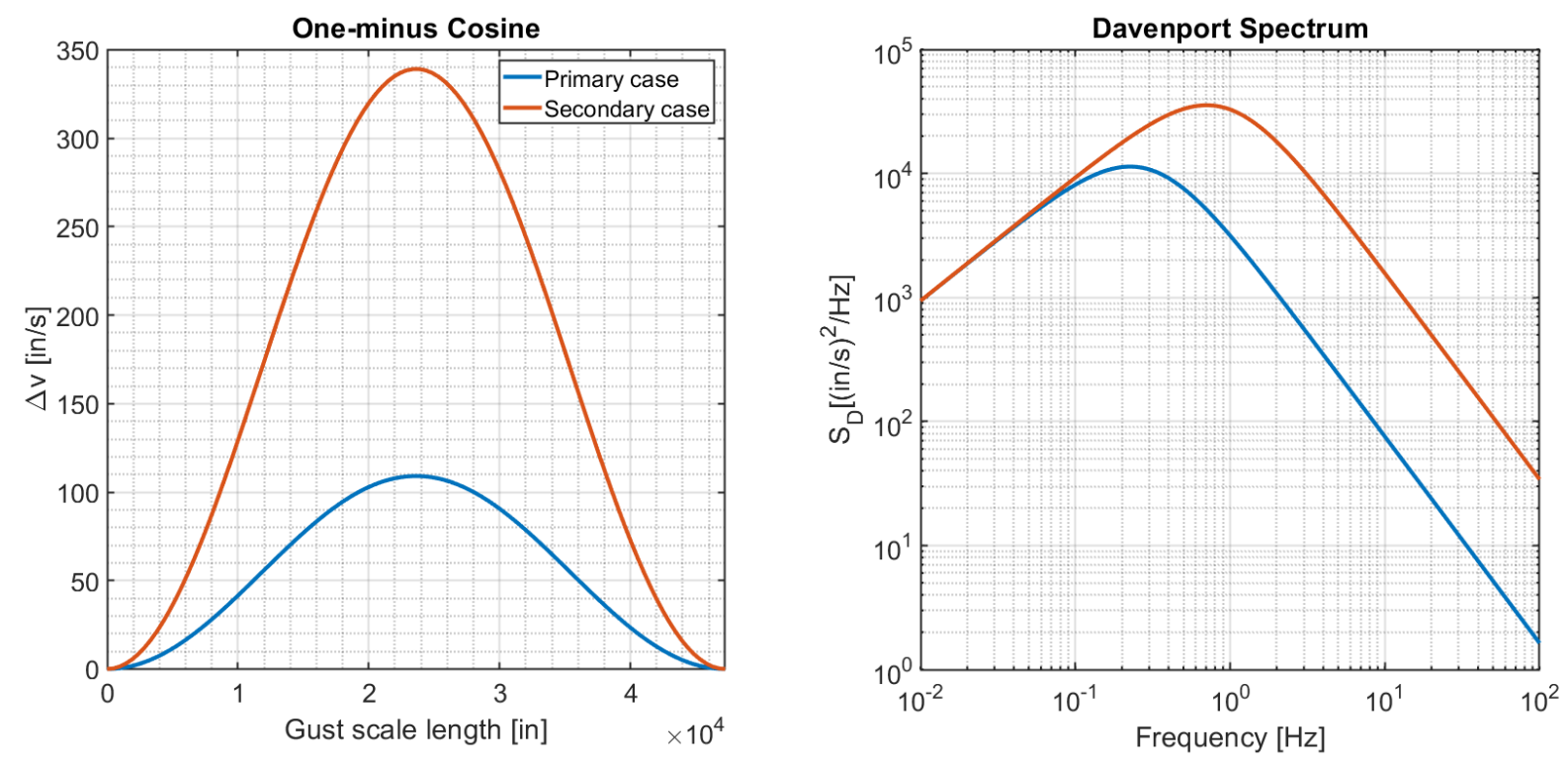

Figure 6.3: TDG and PSD function of the DS considered in the study for 14 operational conditions. The velocities indicated in the color bar are the mean wind speed for the operational scenarios for both gust models

Figure 6.4 presents the frequency and time domain aeroelastic deviation angle responses for both primary and secondary operational cases of the uncontrolled structure, measured at the target node, 
considering the PSD and the TDG models, respectively. For the TDG, after 10 seconds of excitation, the magnitude of response was negligible and therefore truncated. It can be observed that the pointing error in the direction of $\Theta$, even though close to the $\Phi$ response, is the greatest overall for both gust models and operational scenarios considered. This behavior is expected due to the fact that the gust disturbance input was essentially given for the $x$ direction, thus resulting in a response more significant in the referred direction when compared to the others. Table 6.2 summarizes the evaluation of the fitness function of the optimization algorithm for the uncontrolled structure, in order to be taken as reference values for the results section.

Table 6.2: Evaluation of the fitness function for the uncontrolled antenna structure

\begin{tabular}{ccc}
\hline Case ID & PSD & TDG \\
& $U_{\text {fitness }}[\mathrm{deg}]$ & $U_{\text {fitness }}[\mathrm{deg} \times \mathrm{sec}]$ \\
\hline Primary & $5.58 \mathrm{E}-05$ & $2.76 \mathrm{E}-04$ \\
Secondary & $3.78 \mathrm{E}-04$ & $8.39 \mathrm{E}-03$ \\
\hline
\end{tabular}

The frequency response output from Figure 6.4(a-b) presents an accentuated peak estimated at about $2.8 \mathrm{~Hz}$, which is coincident with the first mode shape of the antenna structure. The other frequencies in the spectrum showed magnitudes considerably lower than the one related to the first mode shape, demonstrating its significant participation in the overall response. Therefore, the optimization algorithm should minimize the amplitude of response nearby that frequency, by means of the inclusion of multiple-TMD with optimum parameters. In addition, from Figure 6.4(cd), the time domain response is determined by a summation of a truncated finite number of mode shapes of the structure, and, in this case, it mainly represents the response of the first vibration mode due to its greater dominance. Hence, the focus now is to study the passive control of the 
aeroelastic response by including multiple-TMD devices symmetrically within the antenna structure, investigating optimum design parameters and comparing different placements by using the optimization framework developed in the previous sections.

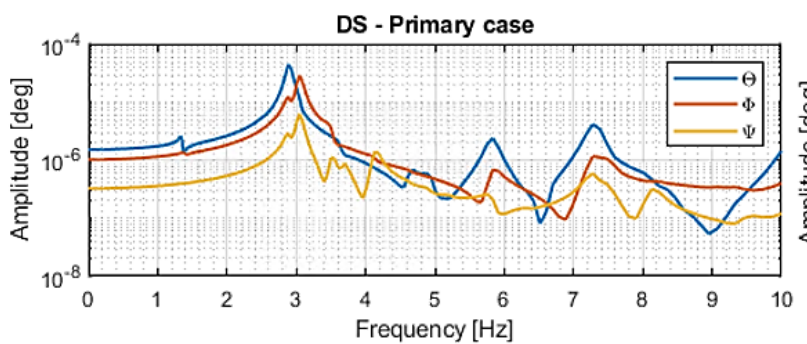

(a)

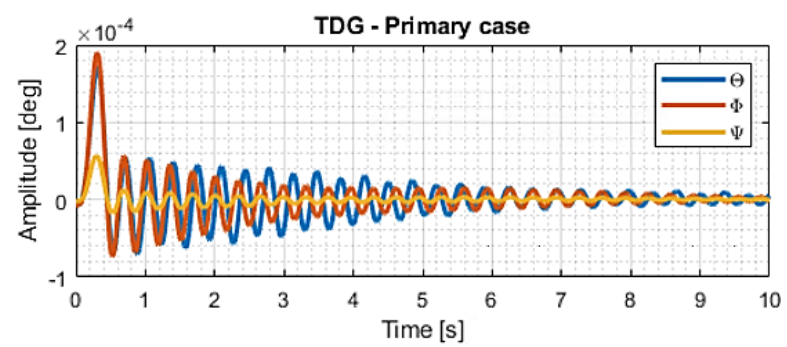

(c)

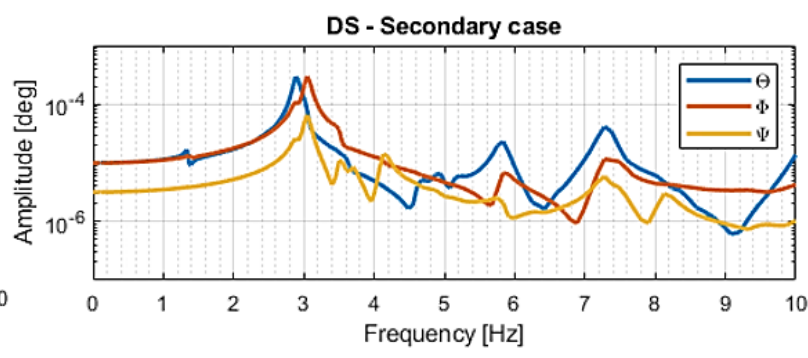

(b)

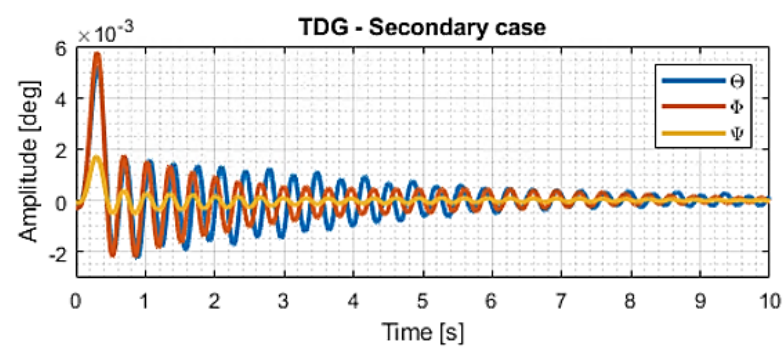

(d)

Figure 6.4: Aeroelastic response of the target node of the uncontrolled antenna structure under the operational cases. PSD wind turbulence for the (a) primary and (b) secondary scenarios. TDG for the (c) primary and (d) secondary scenarios

\subsection{Aeroelastic response attenuation using optimal multiple-TMD}

For the investigation of pointing error minimization of the antenna structure with multiple-TMD control devices, three different configurations of auxiliary masses are proposed. Configuration 1 places the multiple-TMD in the structural grid points of the larger hexagonal shape of the backside truss, totalizing an inclusion of 61 devices. Configuration 2 places the multiple-TMD in the structural grid points of the boom arms, totalizing an inclusion of 29 devices. Finally, 
Configuration 3 merges both of the previous configurations, therefore totalizing an inclusion of 90 devices. A complete description of the multiple-TMD devices nomenclature with respect to the structural grid nodes is presented in Table 6.3 and Figure 6.5.

Table 6.3: Multiple-TMD identification according to the grid point numbering of the FE model of the Earth-based antenna

\begin{tabular}{ccc}
\hline Configuration ID & Multiple-TMD ID & Grid Points ID \\
\hline 1 & {$[1: 61]$} & {$[1: 61]$} \\
2 & {$[1: 29]$} & {$[110: 138]$} \\
3 & {$[1: 90]$} & {$[1: 61,110: 138]$} \\
\hline
\end{tabular}
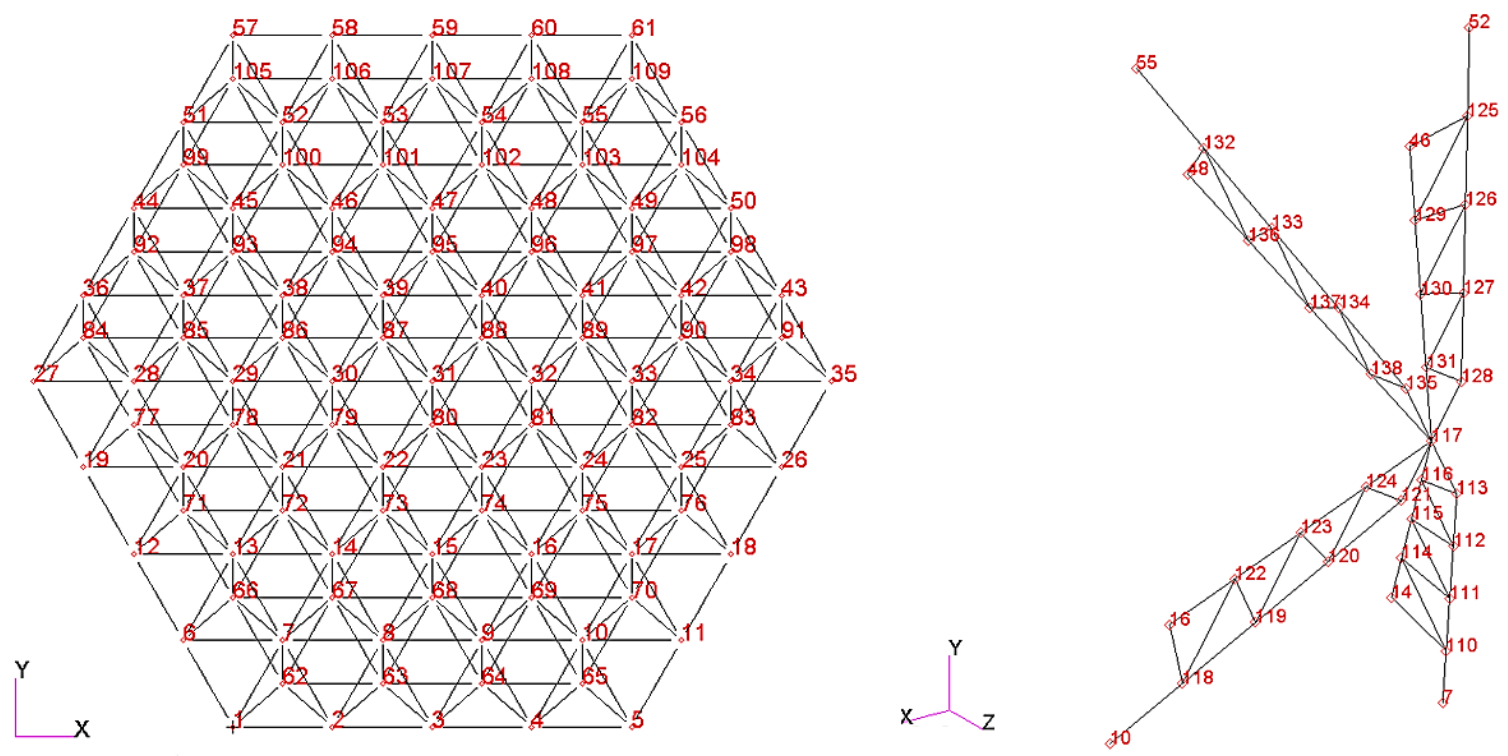

Figure 6.5: Node numbering description for the Earth-based antenna FE model

As it was previously mentioned, all multiple-TMD devices were defined with the same mass, which varies from $0 \%$ to a maximum of $25 \%$ inclusion with respect to the total antenna structure's 
mass. Table 6.4 summarizes the upper and lower boundaries chosen for each individual TMD parameters.

Due to the considerable number of design variables, the parameters of the MOGA must be wisely chosen in order to reduce the convergence time. The following parameters were used in the simulations:

- Maximum number of generations: 30;

- Population size: 300 ;

- Crossover fraction: $80 \%$;

- Elitism probability: 2\%;

- Migration factor: $20 \%$;

- Migration interval: 20;

- Pareto fraction: $35 \%$.

With those parameters, a total number of 9000 analyzes are carried within the MOGA optimization procedure, per configuration. The results for the optimization framework are now presented, considering the operational cases and both models of atmospheric disturbances. The results are given in terms of Pareto fronts, output response functions for the target node, and charts that indicates the optimum values of the multiple-TMD found.

Table 6.4: Design variables bounds for each configuration investigated

\begin{tabular}{ccccccc}
\hline Configuration ID & $n_{T M D}$ & $\begin{array}{c}m_{T M D}^{\max } \\
{[\mathrm{lb}]}\end{array}$ & $\begin{array}{c}c_{T M D}^{\min } \\
{[\mathrm{lbf} . \mathrm{s} / \mathrm{in}]}\end{array}$ & $\begin{array}{c}c_{T M D}^{\max } \\
{[\mathrm{lbf} . \mathrm{s} / \mathrm{in}]}\end{array}$ & $\begin{array}{c}k_{T M D}^{\min } \\
{[\mathrm{lbf} / \mathrm{in}]}\end{array}$ & $\begin{array}{c}k_{T M D}^{\max } \\
{[\mathrm{lbf} / \mathrm{in}]}\end{array}$ \\
\hline 1 & 61 & 5.0 & 0.29 & 285.50 & 2.86 & 2855 \\
2 & 29 & 10.0 & 0.29 & 285.50 & 2.86 & 2855 \\
3 & 90 & 3.3 & 0.29 & 285.50 & 2.86 & 2855 \\
\hline
\end{tabular}




\subsubsection{Frequency domain analysis with the PSD modelled with the Davenport Spectrum}

The optimization procedure presented in the previous chapters was implemented for the gust excitation modelled with the PSD. Representatively, an example of output from the framework is shown in Figure 6.6, where the pointing error and mass inclusion are minimized for the primary operational case. In the Figure 6.6(a) it is possible to observe that the decrease in the pointing error is associated with a direct increase in the mass inclusion in the antenna, therefore establishing a trade-off problem. The utopian point indicated encompasses the extremes of the Pareto front, hence considering all individuals obtained at the convergence of the optimization algorithm.

According to the project demands, it is possible to select different best individuals, by means of the use of the weight function previously described. When they are taken as unitary values, both objectives have the same relevance to define the best individual, which is the case of this analysis. Hence, the best individual found, for the referred operational case and configuration selected, allows a reduction of the pointing error in $62 \%$, while requiring a total mass inclusion of $5.9 \%$. In Figure 6.6(b), all individuals from the Pareto front were plotted, and it is observable that the maximum mass inclusion provided a significant attenuation of the first resonance frequency, reaching a reduction of the pointing error of $87.6 \%$, with total mass inclusion of $23.9 \%$. Another relevant observation is that all angles had their response attenuated by the inclusion of the multipleTMD, therefore demonstrating the capability of MDOF aeroelastic response mitigation of those devices. Such fact was also observed in all other operational cases and configurations investigated. 


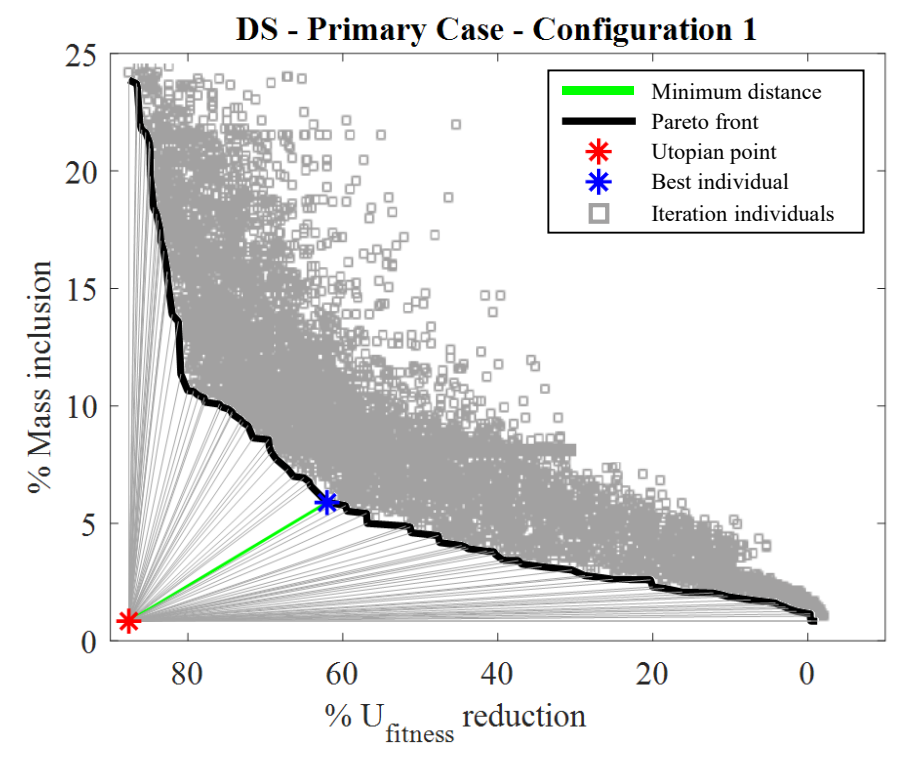

(a)
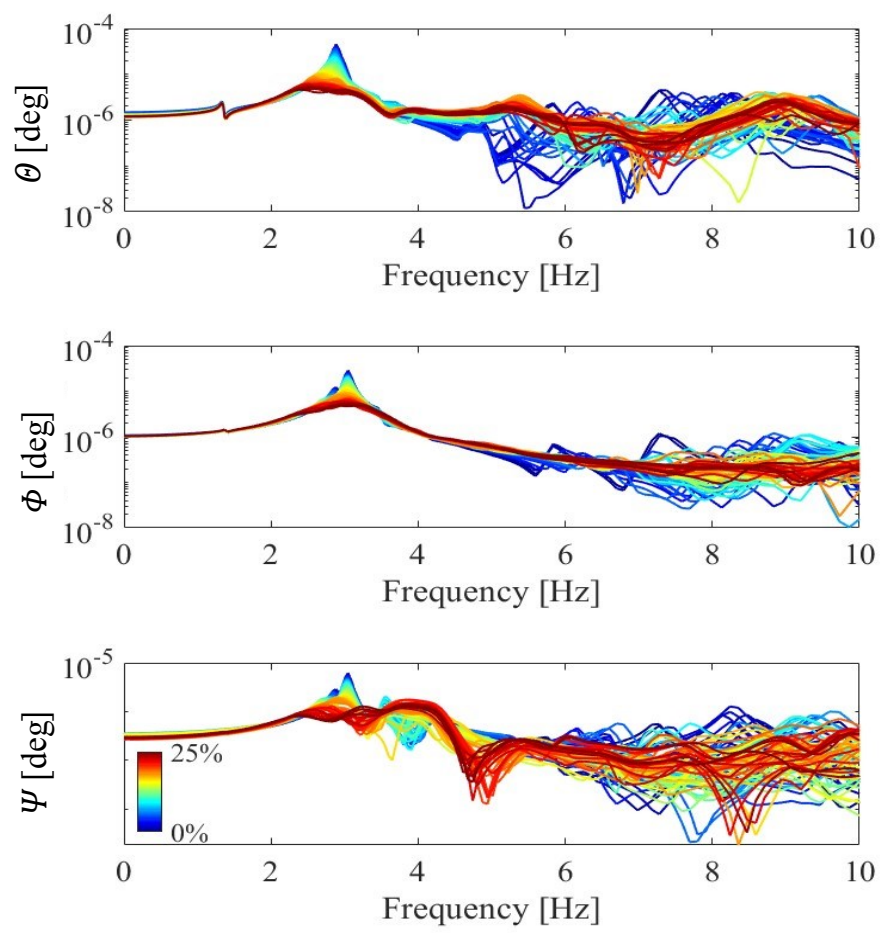

(b)

Figure 6.6: Pointing error minimization using the multiple-TMD under the configuration 1 for the primary operational case modelled with the PSD function of the DS. (a) Multiobjective Pareto front of all individuals analyzed, indicating the utopian point and the best 
individual. (b) Frequency response functions of the deviation angles for each respective direction, where the color bar indicated represents the percentage of mass inclusion in the antenna

Table 6.5: Pareto front values for the best individuals under the DS gust excitation model

\begin{tabular}{ccccc}
\hline & \multicolumn{2}{c}{ Primary case } & \multicolumn{2}{c}{ Secondary case } \\
\cline { 2 - 5 } $\begin{array}{c}\text { Configuration } \\
\text { ID }\end{array}$ & $\begin{array}{c}\text { \% Mass } \\
\text { inclusion } \\
{[\mathrm{lb}]}\end{array}$ & $\begin{array}{c}\text { \% } U_{\text {fitness }} \\
\text { reduction }[\mathrm{deg}]\end{array}$ & $\begin{array}{c}\text { \% Mass } \\
\text { inclusion } \\
{[\mathrm{lb}]}\end{array}$ & $\begin{array}{c}\% U_{\text {fitness }} \\
\text { reduction } \\
{[\mathrm{deg}]}\end{array}$ \\
\hline 1 & 5.9 & 62.0 & 4.6 & 51.6 \\
2 & 2.9 & 43.0 & 3.2 & 50.1 \\
3 & 4.9 & 60.0 & 4.0 & 39.5 \\
\hline
\end{tabular}

In order to compare the Pareto fronts obtained with different configurations under both operational scenarios, Figure 6.7 is presented. The first clear observation is that some of the configurations considered were not able to reach the upper bound of $25 \%$ mass inclusion. This is attributed to the fact that for higher percentages of mass inclusion were not resulting in feasible optimum values for the Pareto front, thus returning an increase in the pointing error. In addition, it possible to conclude that the Configuration 1 presented the overall best performance for both operational cases, providing the best trade-off between mass inclusion and pointing error minimization. Coincidently, such observation was also valid for the TDG simulations, as it will be shown in the next session. A summary of the Pareto front values for the best individuals of Figure 6.7 is presented in the Table 6.5. 


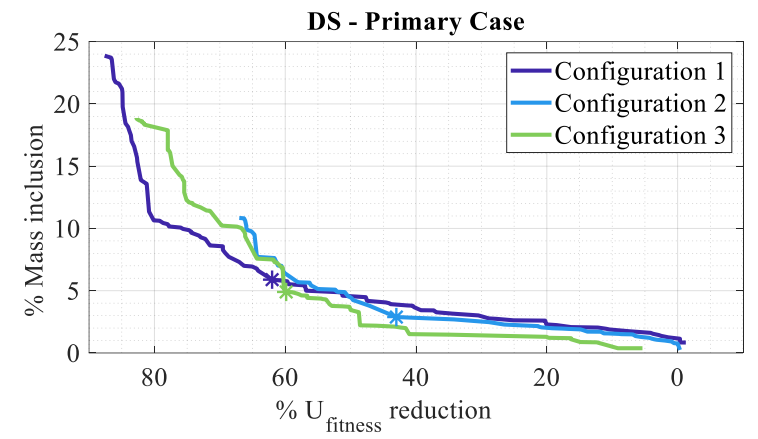

(a)

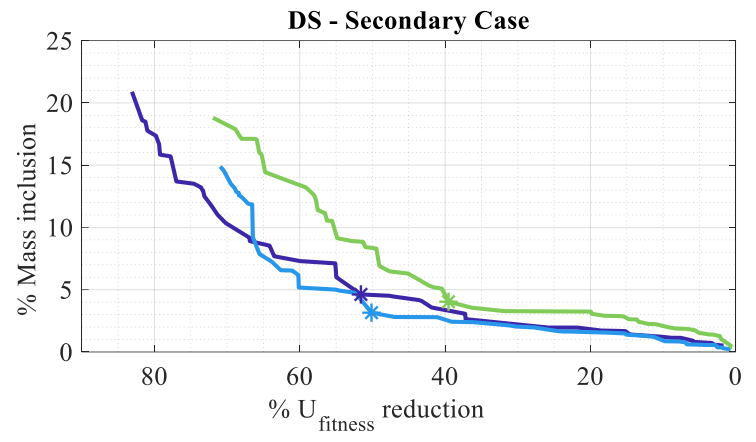

(b)

Figure 6.7: Comparison of Pareto fronts between all configurations investigated in the study for both operational scenarios modelled with the DS. (a) Primary and (b) Secondary cases
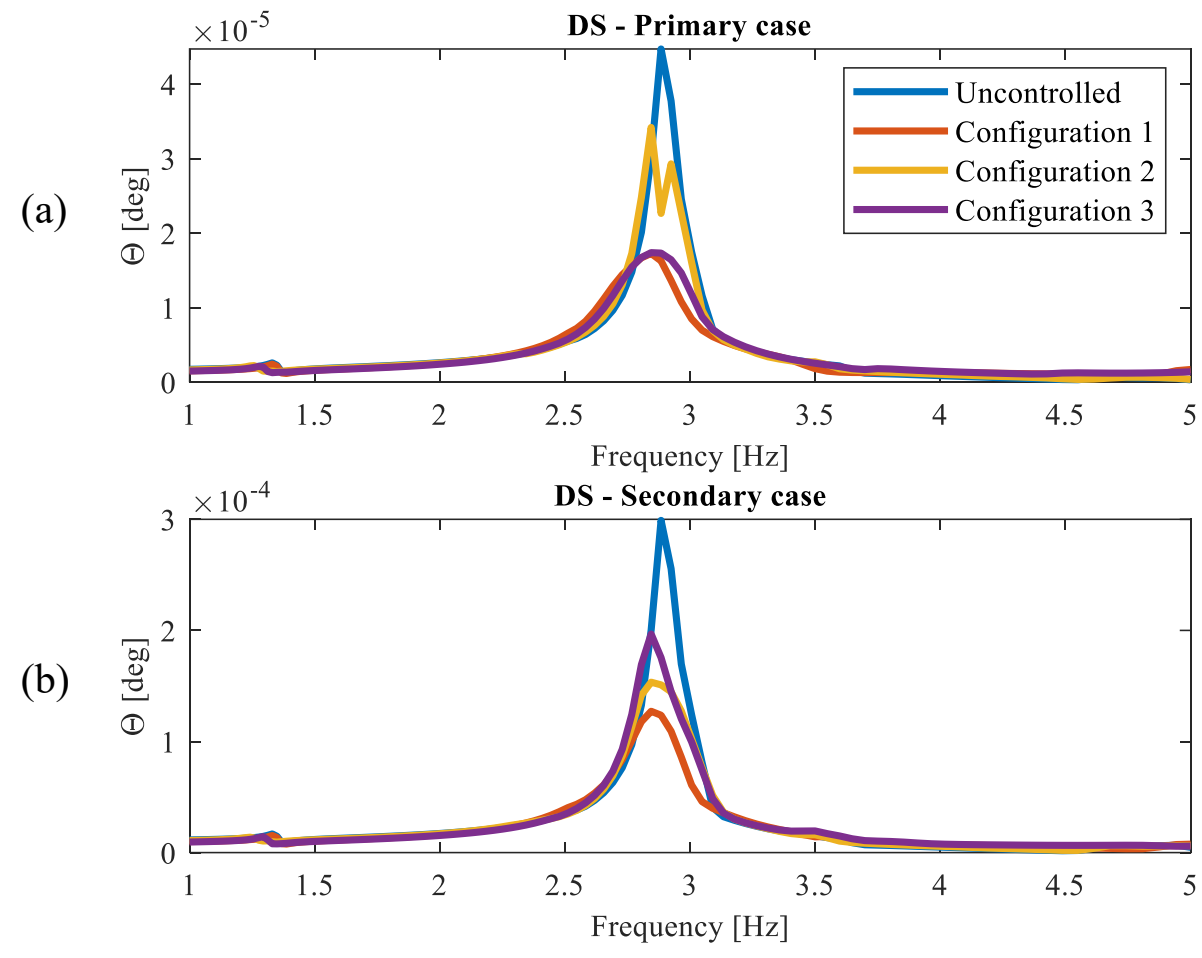

Figure 6.8: Comparison of the frequency response functions, for the $\Theta$ DOF, considering all best individuals of each configuration investigated, obtained with the utopian point method. The (a) graph presents the results for the primary operational case modelled with the DS, while the (b) graph represents the secondary operational case 
Figure 6.8 depicts the output response for the dominant DOF angle, $\Theta$, for all best individuals of the different configurations under the two operational scenarios, compared with the uncontrolled response of the antenna, hence demonstrating the better attenuation capability of Configuration 1. It is known that the placement of multiple-TMD is more effective in regions of higher displacement, therefore providing better attenuation capabilities [23]. The placement of the multiple-TMD in the Configuration 1 and Configuration 3 presented advantages with respect to the Configuration 2 due to the fact that they are mainly located in grid points of maximum displacement of the first resonance mode shape from the antenna structure, therefore resulting in superior attenuating capabilities.

\subsubsection{Time domain analysis with the One-minus cosine gust model}

Now, the two operational cases are applied to the antenna structure considering the TDG model, and the performance of each multiple-TMD configuration are compared. Similarly, as in the previous gust model analysis, a representative output from the framework is presented, where the concurrent minimization of mass inclusion and area underneath the curve of pointing error with respect to time, for the Configuration 1, considering the secondary operational case are depicted in Figure 6.9(a). The choice of the second objective is based on the fact that a smaller area under the curve would provide a faster attenuation to the gust signal, which presents a behavior like an impulse signal, due to the short duration. Figure 6.9(b) displays the deviation angle in terms of the RSSQ, therefore providing the net displacement of the DOF angles. As it is presented, an alike behavior of direct proportionality between the mass inclusion and the reduction of the $U_{\text {fitness }}$ was

characterized, as in the case of the DS model, achieving an overall maximum of $58.5 \% U_{\text {fitness }}$ minimization with $23.6 \%$ added mass in the antenna structure. 


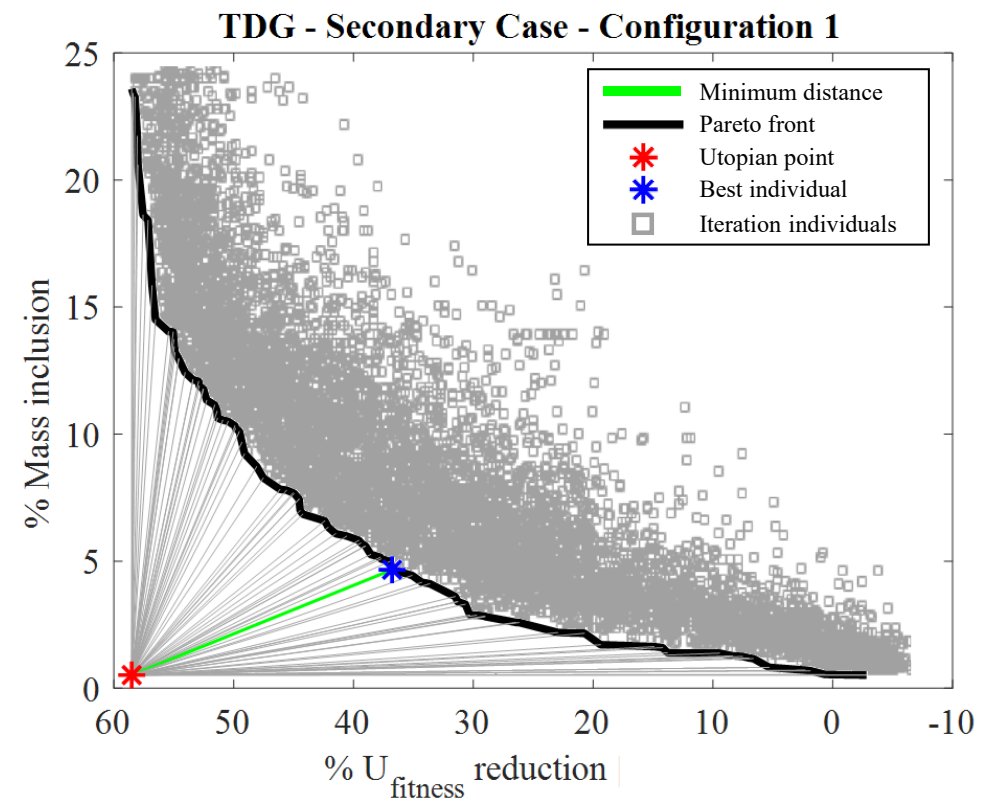

(a)

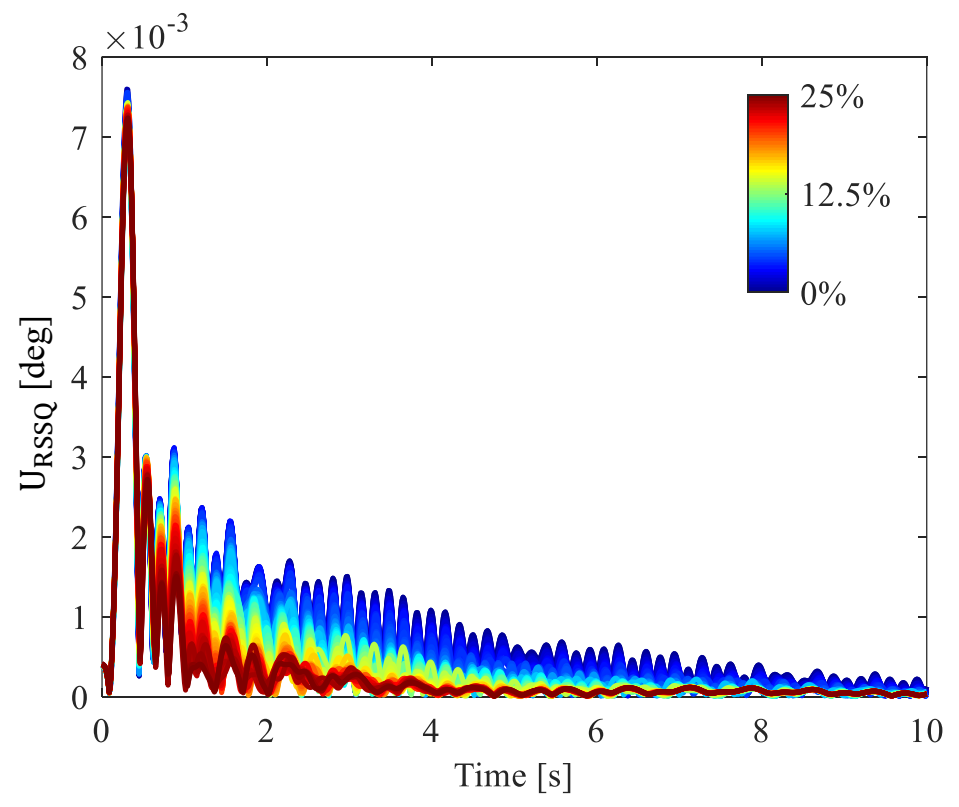

(b)

Figure 6.9: Pointing error minimization using the multiple-TMD under the configuration 1 for the secondary operational case modelled with the TDG. (a) Multi-objective Pareto front of all individuals analyzed, indicating the utopian point and the best individual. (b) Time 
response functions of the RSSQ deviation angle for each respective direction, where the color bar indicated represents the percentage of mass inclusion in the antenna

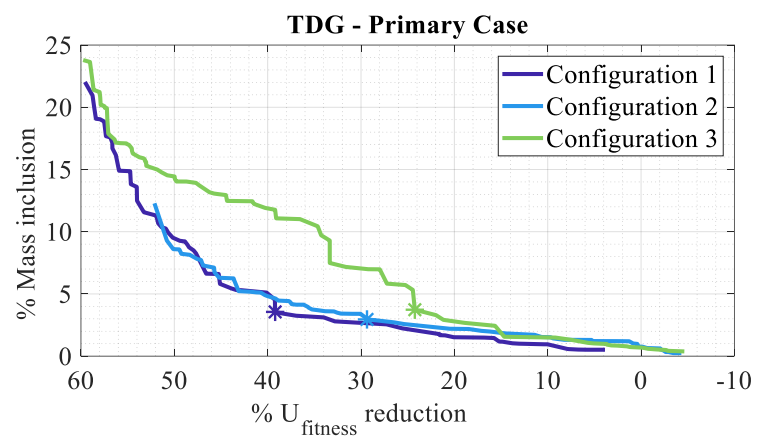

(a)

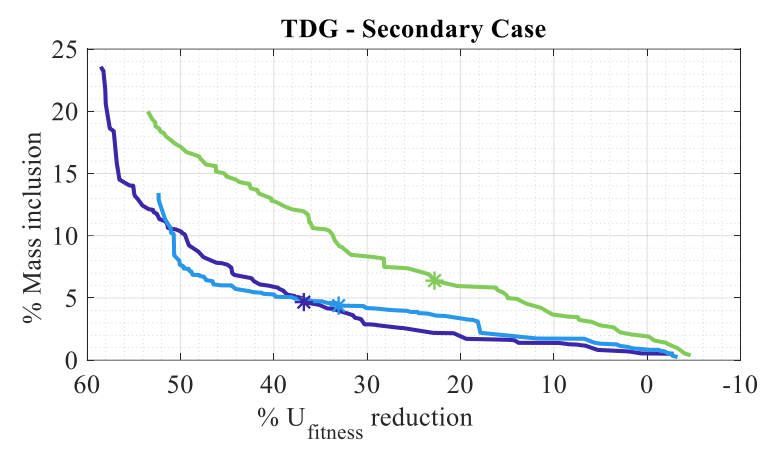

(b)

Figure 6.10: Comparison of Pareto fronts between all configurations investigated in the study for both operational scenarios modelled with the TDG. (a) Primary and (b) Secondary cases

By applying the utopian point method to the optimal individuals generated from the MOGA framework, one can determine the best sets from the Pareto frontiers considering all configurations and operational cases, as presented in Figure 6.10. Observing the graphs depicted, it is possible to conclude that while maintaining a relatively similar mass inclusion percentage, Configuration 1 presented the highest reduction in $U_{\text {fitness }}$ for both configurations, and operational cases investigated. The objectives values are summarized in the Table 6.6. Moreover, the performance of the configurations is displayed in Figure 6.11, where the time response for the TDG excitations is compared. It can be seen that the inclusion of the multiple-TMD devices provide a considerable attenuation of the uncontrolled signal between the time interval presented, where the Configuration 1 resulted in the best deviation angle reduction performance. 
(a)

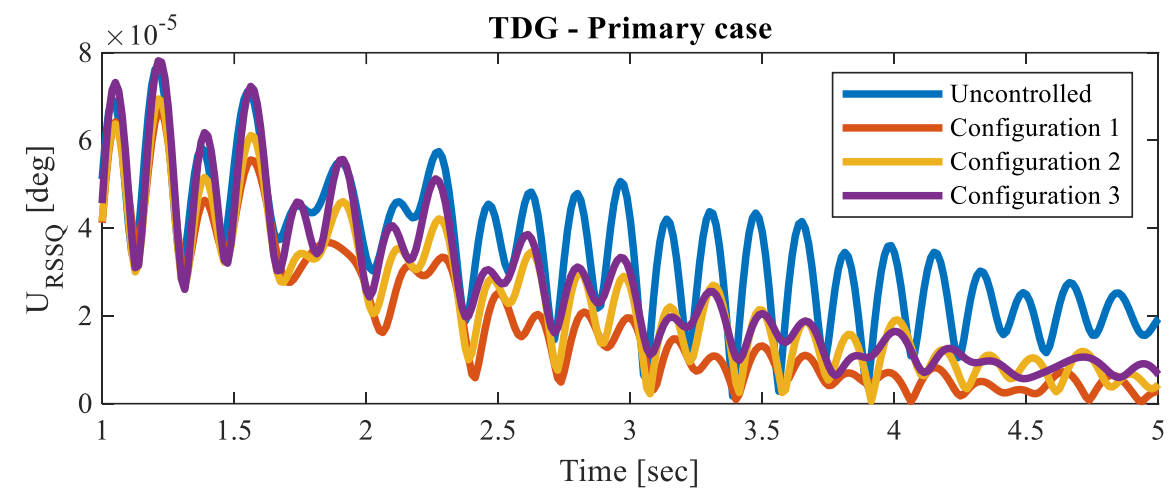

(b)

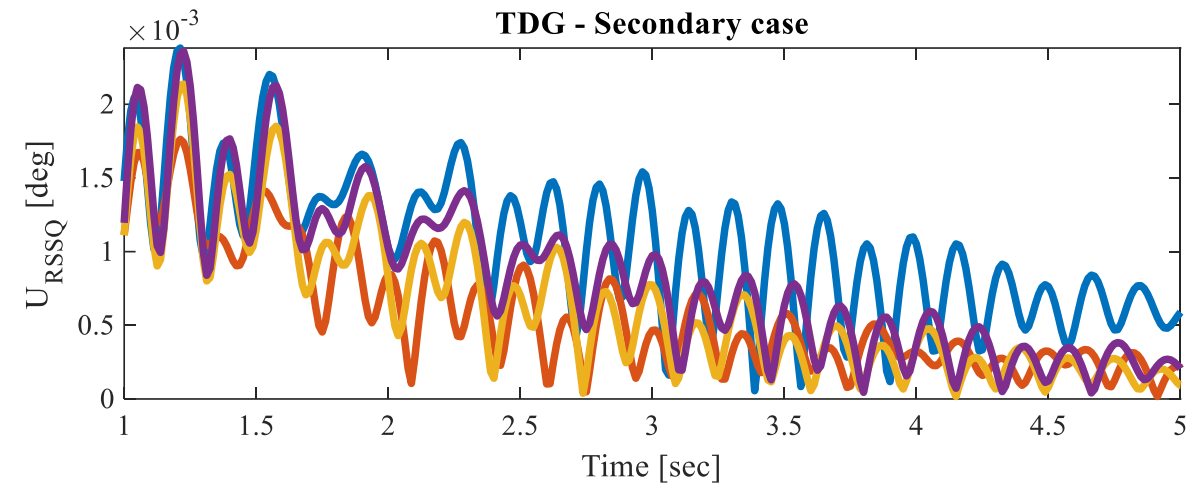

Figure 6.11: Comparison of the time response functions, for the RSSQ deviation angle, considering all best individuals of each configuration investigated, obtained with the utopian point method. The (a) graph presents the results for the primary operational case modelled with the TDG, while the (b) graph represents the secondary operational case

Table 6.6: Pareto front values for the best individuals under the TDG gust excitation model

\begin{tabular}{ccccc}
\hline & \multicolumn{2}{c}{ Primary case } & \multicolumn{2}{c}{ Secondary case } \\
\cline { 2 - 5 } $\begin{array}{c}\text { Configuration } \\
\text { ID }\end{array}$ & $\begin{array}{c}\text { \% Mass } \\
\text { inclusion } \\
{[\mathrm{lb}]}\end{array}$ & $\begin{array}{c}\% U_{\text {fitness }} \text { reduction } \\
{[\mathrm{deg} \times \mathrm{sec}]}\end{array}$ & $\begin{array}{c}\text { \% Mass } \\
\text { inclusion } \\
{[\mathrm{lb}]}\end{array}$ & $\begin{array}{c}\% U_{\text {fitness }} \\
\text { reduction } \\
{[\mathrm{deg} \times \mathrm{sec}]}\end{array}$ \\
\hline 1 & 3.6 & 39.2 & 4.7 & 36.8 \\
2 & 3.0 & 29.4 & 4.4 & 33.0 \\
3 & 3.7 & 24.2 & 6.4 & 22.8 \\
\hline
\end{tabular}




\subsection{Summary of main findings}

From the analysis performed in this section, it was found that the overall best performance was achieved with the multiple-TMD under Configuration 1, therefore the optimal values for the mass, damping, and stiffness are given respectively in Table 6.7 and Figure 6.12. As it is expected, the multitude of boundary conditions explored returned non-unique optimal values for the multipleTMD, among the cases analyzed. This is an inherent characteristic of this type of passive control device, such that the optimal parameters strongly depend on the wind disturbance velocity for different operational conditions [44].

Table 6.7: Summary of the best values for the multiple-TMD masses for the Configuration 1 under the operational scenarios modelled with the DS and TDG

\begin{tabular}{cc}
\hline Operational case & Optimal Values for $\mathbf{m}_{T M D}[\mathrm{lb}]$ \\
\hline DS - Primary & 1.14 \\
TDG - Primary & 0.69 \\
DS - Secondary & 0.90 \\
TDG - Secondary & 0.90 \\
\hline
\end{tabular}

Hence, for both atmospheric disturbance models, the overall best individual was given by the Configuration 1, which presents the multiple-TMD placed in the backside truss of the antenna. This was an expected result due to the fact that the placement of multiple-TMD is more effective in regions of higher displacements, therefore providing better vibration attenuation capabilities. By utilizing the multiple-TMD under Configuration 1, it was possible to characterize a reduction in the pointing error of the antenna by $62.0 \%$ and $51.6 \%$ for the primary and secondary operational 
cases considering the DS model, respectively, and $39.2 \%$ and $36.8 \%$ for the primary and secondary operational cases considering the TDG model, respectively, hence demonstrating the excellent performance of the implementation of such passive control method.

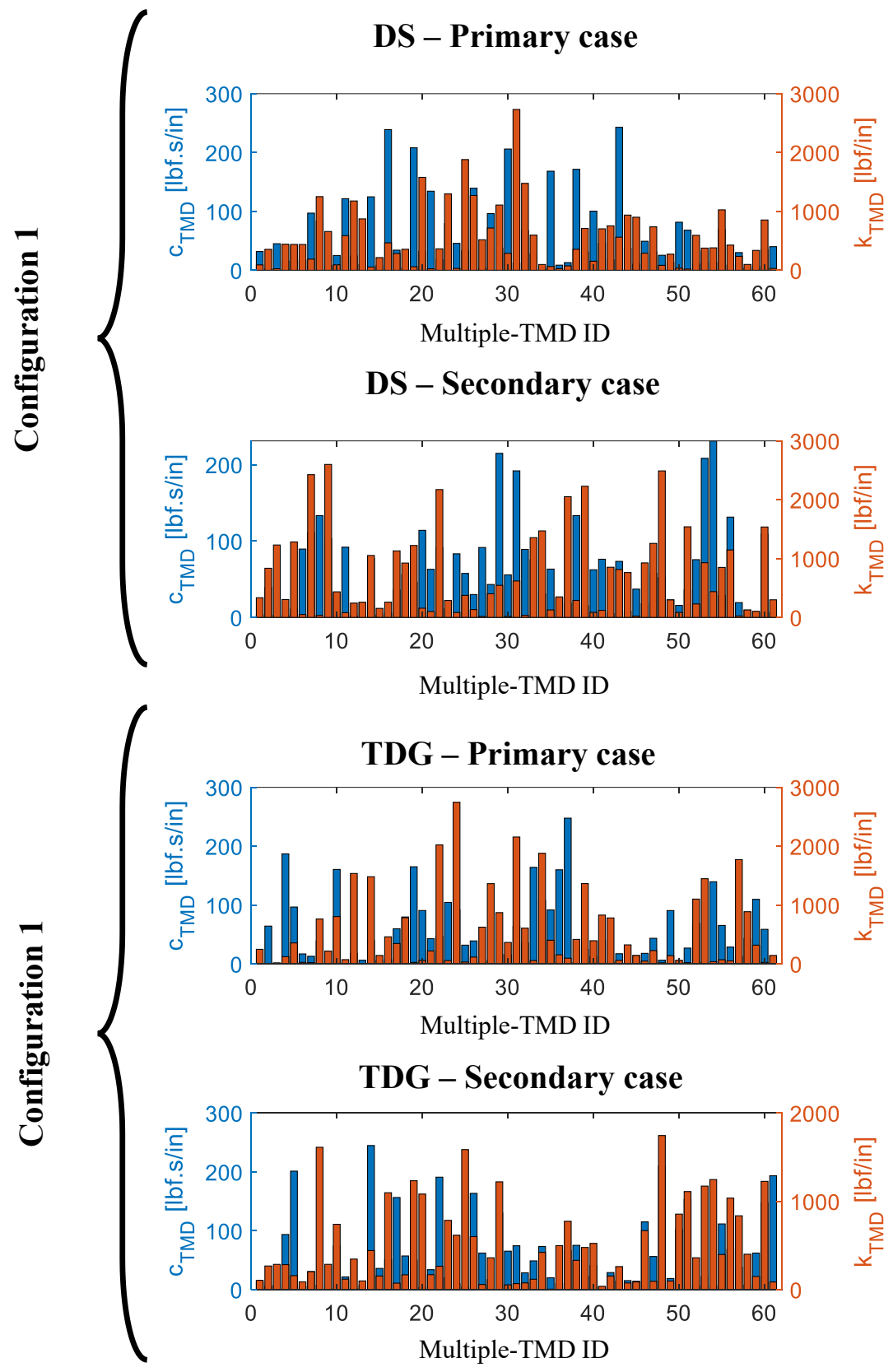

Figure 6.12: Summary of the multiple-TMD Configuration 1 optimal damping and stiffness values, considering both operational cases modelled with the (a-b) DS and the (c-d) TDG 


\section{Chapter 7: Case study II - Pointing error reduction of a 12m Earth-based radio VLBI antenna model}

\subsection{Introduction}

This chapter presents the application of the developed framework to an industrial model of a $12 \mathrm{~m}$ VLBI antenna, provided by InterTronic Solutions Inc. Similarly to the Case Study I, it is intended to minimize the pointing error of the structure when subjected to aerodynamic gusts under several different operational scenarios. It will be discussed aspects of the simulation setup, such as the construction of the new reduced order model created, in order to decrease the computation time of the iterations, MOGA parameters and strategies utilized, and results obtained from the framework implementation.

\subsection{Model order reduction of the VLBI antenna structure}

When iteratively analyzing finite element models, the computation time is a factor that must be taken into consideration. The use of superelements in MSC NASTRAN ${ }^{\circledR}$ is a strategy to not only analyze large models, but also to increase the efficiency in terms of completion time of each run, therefore allowing the designer to solve more iterations or design cycles.

This gain in computational efficiency is possible due to the fact that the model is analyzed through an incremental process, where the constitutive FE matrices are previously calculated, reduced and substructured, if necessary. This effect results in greatly reducing the computation time, in the order from 2 to 30 times faster than non-superelement models [130]. Hence, this characteristic makes the use of this strategy very favorable when analyzing aerospace structures, where FE models often have a high number of degrees of freedom to be processed. 


\subsubsection{Superelement implementation and ROM consistency verification}

The superelements strategy is commonly employed in parallel with structural model order reduction methods, such as the Craig-Bampton reduction method (CBRM) [131]. The purpose of use of this method is essentially to determine and remove degrees of freedom that present lower contribution to the final structural response, therefore reducing the size of the matrices to be analyzed. In the CBRM, the primary dynamic system is portioned into different subsystems, and each associated displacement vectors are expressed with a combination of physical and generalized coordinates, where a reduced set of normal modes are used to represent the model. The reduced stiffness and mass matrices of the structure are then determined by a projection in the constructed reduced subspace of the displacement modes, and thus accordingly assembled for the subsystems considered. The derivation of the constitutive equations of the CBRM is out of scope of this thesis, and the reader is addressed to the interesting work performed by Thomas et al. [132] to have an in-depth understanding of the mathematical procedure and application of the method.

The definition of a superelement essentially requires two main steps:

1. The first step is the creation of the external superelement matrices, by defining the type of matrix output (EXTSEOUT case control command) and designating the representative grid points and degrees of freedom that the boundary matrices will refer to (ASET bulk entry). These representative grid points are the ones which constraints or loads are applied to, or where the superelement connects to either other external superelements or a residual structure present in the simulation; 
2. The second step is the assembly of the external superelements (in case of having multiple defined) and residual structure in a unique model, i.e. in a separate solution file, and then normally run the model to obtain the response at desired nodes.

Regarding the first step mentioned, MSC NASTRAN® ${ }^{\circledR}$ allows the user to generate the boundary matrices in different formats, and whether to write a connectivity file between the parent model and the superelement created [130]. For this work, the default storage format for the boundary matrices is utilized, and they are defined by three files: ".MASTER", ".DBALL" and ".ASM". The ".MASTER" file contains the directory information of the simulation database, which are Data Base-sets (DB-sets) and their physical file names, a directory of projects, versions, data blocks, parameters, Direct Matrix Abstraction Program (DMAP) source and object files. The ".DBALL" file contains the DMAP data blocks with the actual physical information about the model and the Output Transformation Matrices (OTM) for the order reduction. The "ASM" file contains connectivity data for each external superelement.

After the assembly of the SE, and particularly in the case of a dynamic analysis, it is important to verify the consistency of the ROM with the parent detailed FE model, in order to assure effective correlation between mode shapes and natural frequencies found. This verification can be performed by means of the percentage error calculated between natural frequencies and the Modal Assurance Criteria (MAC) [133]. The percentage error is assessed with Equation (7.1),

$$
\operatorname{error}[\%]=\left|\frac{\omega_{R O M-1}-\omega_{R O M-2}}{\omega_{R O M-1}}\right| \times 100
$$

where $\omega_{R O M-1}$ and $\omega_{R O M-2}$ are the referred natural frequencies of the ROM and the parent model. 


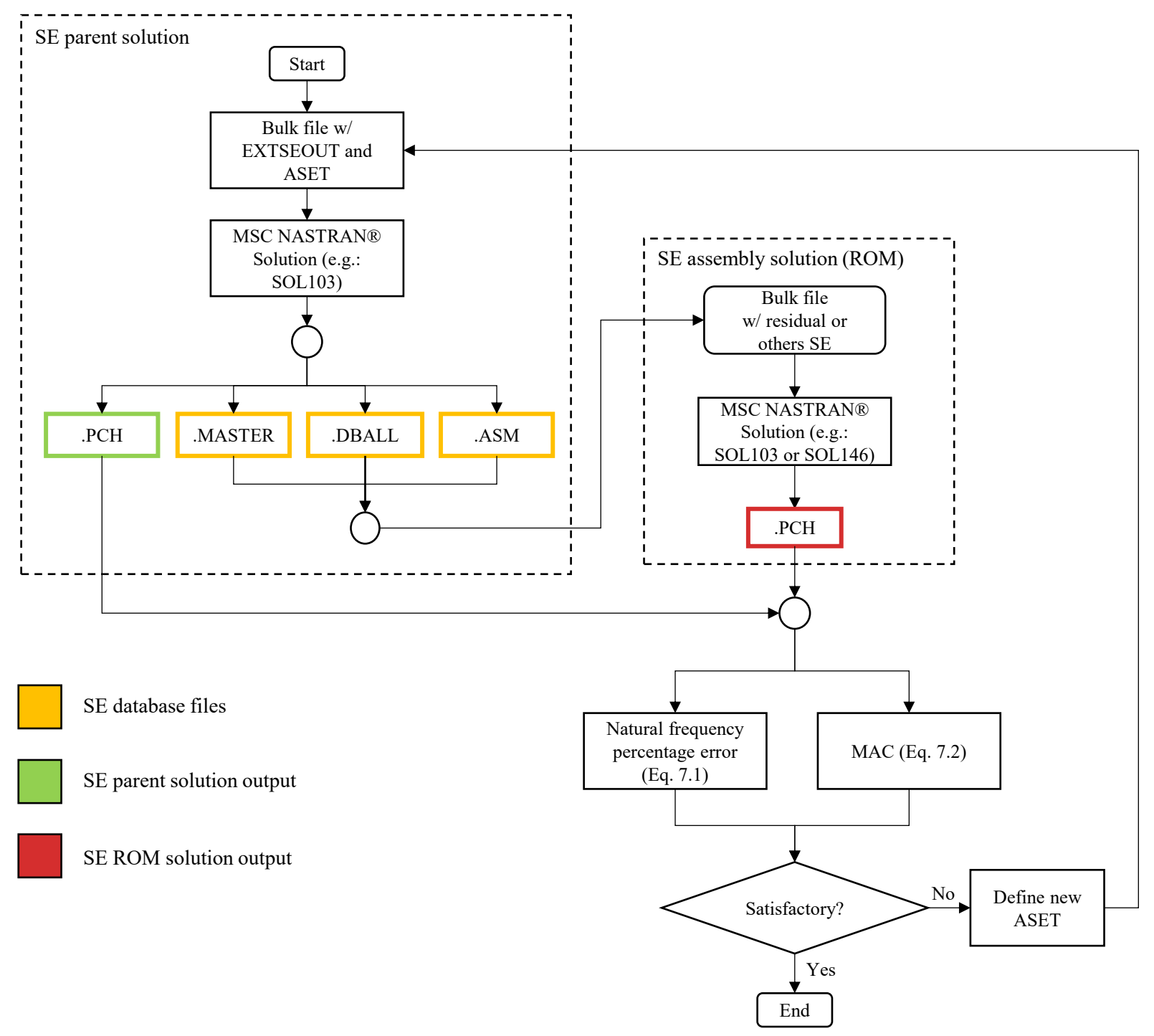

Figure 7.1: External superelement creation and verification of ROM consistency

The MAC is formulated as in Equation (7.2). The correlation between the models considered, assessed by this method should result in an identity matrix, thus indicating an identical comparison, and present values close to zero when different mode shapes are compared.

$$
\text { MAC }=\frac{\left|\left\{\boldsymbol{\Phi}_{R O M-1}\right\}_{r}^{T}\left\{\boldsymbol{\Phi}_{R O M-2}\right\}_{S}\right|^{2}}{\left(\left\{\boldsymbol{\Phi}_{R O M-1}\right\}_{r}^{T}\left\{\boldsymbol{\Phi}_{R O M-1}\right\}_{r}\right)\left(\left\{\boldsymbol{\Phi}_{R O M-2}\right\}_{S}^{T}\left\{\boldsymbol{\Phi}_{R O M-2}\right\}_{S}\right)}
$$


In Equation (7.2), $\left\{\boldsymbol{\Phi}_{R O M-1}\right\}_{r}$ is the r-th mode shape of the ROM 1 considered, and $\left\{\boldsymbol{\Phi}_{R O M-2}\right\}_{S}$ is the s-th mode shape of the ROM 2 considered. Figure 7.1 depicts the procedure for a superelement definition verification of its validity, using the steps previously described. The criteria to categorize a solution as "satisfactory" utilized for the work was RMS of the percentage error lower than $1 \%$ and MAC diagonality near the unitary value.

\subsubsection{Final reduced order model creation}

In order to carry the optimization procedure with the FE model of the $12 \mathrm{~m}$ VLBI antenna, it is necessary to numerically reduce the Detailed FE model (DFEM) provided by the industrial partner, which is depicted in Figure 7.2. The previous work from Nieto et al. [1] subjected this antenna model to an order reduction procedure, being able to considerably minimize the computational cost of the simulation. However, the computation time was still relatively high when considering its implementation in the optimization framework, specially using MOGA as the optimizer method, since it requires several generations and a high population number to achieve the convergence of the results.

The criteria utilized to further reduce the model generated by Nieto et al. [1] is based in the elimination of grid points from the main ASET card that do not participate in the following components of the simulation:

- Boundary condition location (i.e., SPC grid points);

- Aerodynamic panels grid points (DLM panels formation);

- Structural spline grid points;

- Truss-structure of the boom arms that connect the primary and secondary reflector; 
- Monitoring grid points that were defined in the work of Nieto et al. [1] (those are specifically related to the reference and target nodes that are used to assess the pointing accuracy of the antenna).

Thus, any grid point that does not satisfy at least one of these criteria is then removed from the analysis set for the creation of the superelement constitutive matrices. After this procedure was executed, the number of grid points decreased from 1328 to 573, representing a 57\% decrease in the model size. Figure 7.2 depicts the evolution of the FE model of the VLBI antenna structure.

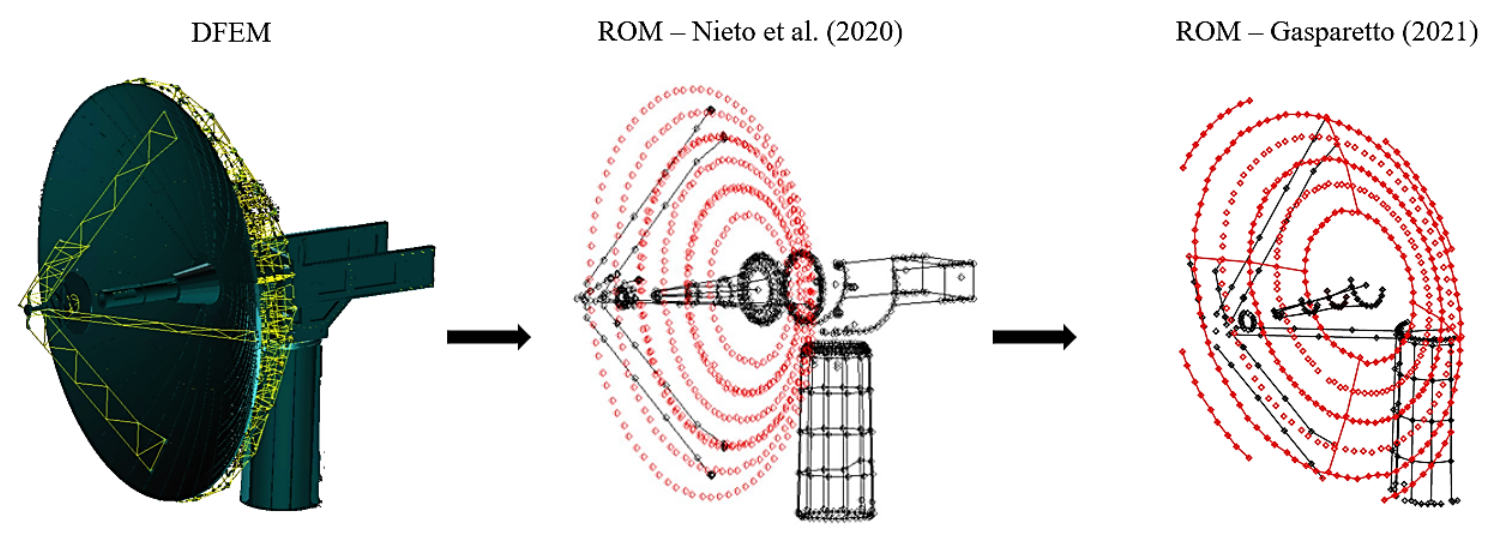

Figure 7.2: InterTronic Solutions 12m VLBI antenna FE model evolution

The percentage error calculated between the first 25 eigenfrequencies of the flexible modes from the two ROMs considered herein are compared in Figure 7.3. The percentage error is assessed with Equation (7.1), where $\omega_{R O M-1}$ and $\omega_{R O M-2}$ are the eigenvalues from the ROM developed by Nieto et al. (2020) and Gasparetto (2021), respectively. From Figure 7.3 it can be seen that the errors achieved are very low, with RMS evaluated as $0.0385 \%$, which indicates good proximity between the eigenvalues determined. 
In order to evaluate the correlation between the ROMs considered, the MAC is utilized to compare the first 25 eigenvalues and indicate the consistency of the solution. The MAC is given as in Equation (7.2), where $\left\{\boldsymbol{\Phi}_{R O M-1}\right\}_{r}$ is the r-th mode shape of the ROM developed by Nieto et al. [1], and $\left\{\boldsymbol{\Phi}_{R O M-2}\right\}_{S}$ is the s-th mode shape of the ROM implemented in this work.

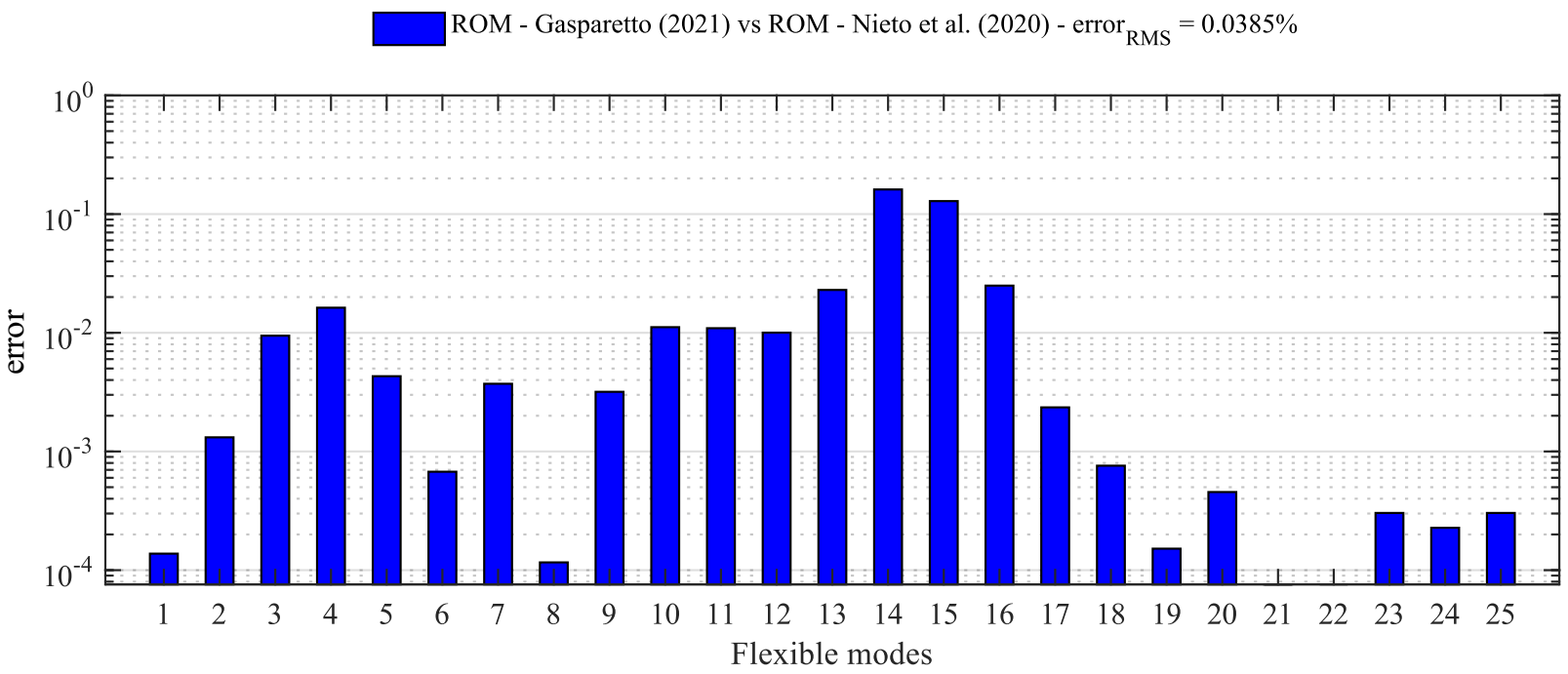

Figure 7.3: Percentage error evaluated for the natural frequencies of the first 25 flexible modes for the ROM - Gasparetto (2021) and Nieto et al. (2020)

As it can be seen from Figure 7.4, the normal modes of the new reduced model implemented by the author present good correlation with the model developed by Nieto et al. [1]. A small loss of correlation was observed between the mode shapes 17 and 18, which represent an inversion of the order of appearance of such mode shapes. Nevertheless, despite this inversion, these mode shapes still presented full consistency with each other, as indicated by the MAC matrix values.

The solution time comparison for both models is presented in the Table 7.1. The simulations were benchmarked in a workstation with an Intel ${ }^{\circledR}$ Core ${ }^{\mathrm{TM}}$ i7-7700K CPU $4.20 \mathrm{GHz}$ with 4 cores and RAM 32 GB. It was possible to reduce the computation time of the SOL103 by $73 \%$ and the 
SOL146 by $66 \%$, from the original ROM. Thus, given the considerable reduction of computation cost achieved for the new ROM and its proven fidelity to the original ROM and DFEM, it will be utilized in the further analysis to minimize the pointing error caused by aerodynamic excitations.

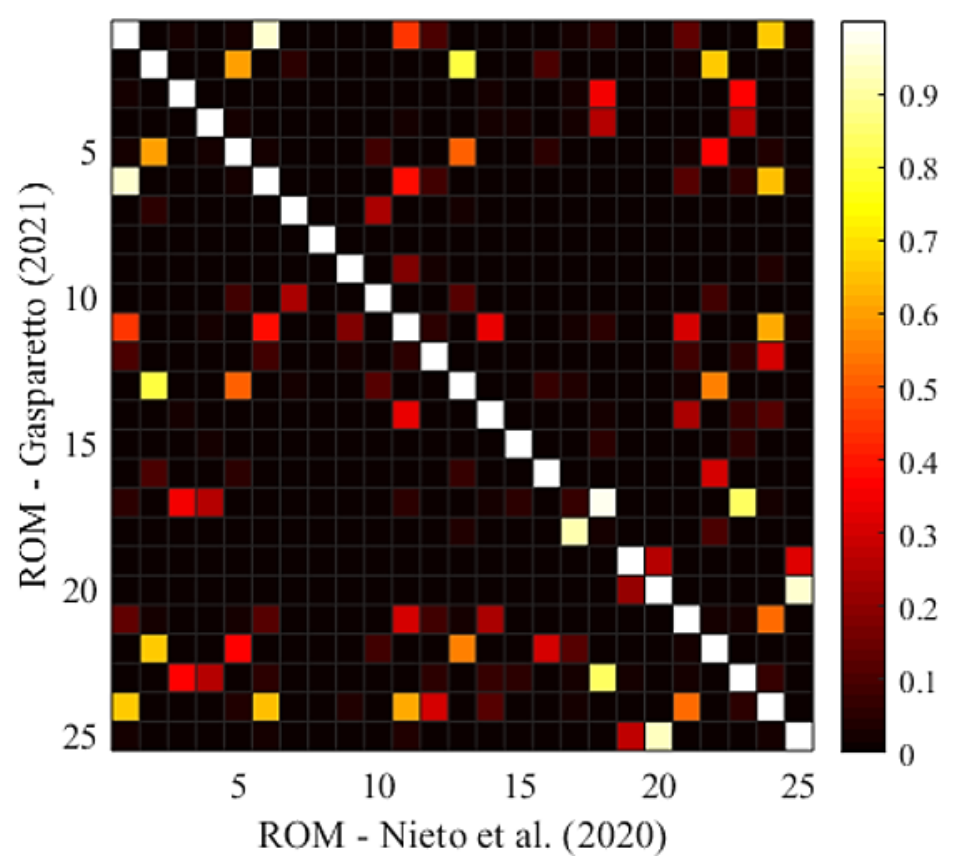

Figure 7.4: First 25 normal modes correlation between the ROM - Gasparetto (2021) and Nieto et al. (2020)

Table 7.1: Computational cost comparison

\begin{tabular}{ccc}
\hline \multirow{2}{*}{ Model } & \multicolumn{2}{c}{ Computational time analysis - First 30 flexible modes (sec) } \\
\cline { 2 - 3 } & Normal modes (SOL103) & Dynamic aeroelasticity (SOL146) \\
\hline DFEM [38] & 1638.0 & 2352.6 \\
ROM - Nieto et al. [1] & 65.4 & 186.4 \\
ROM - Gasparetto (2021) & 17.7 & 61.0 \\
\hline
\end{tabular}




\subsection{Uncontrolled VLBI antenna structure aeroelastic response}

As discussed in Nieto et al. [1], the steady-state aeroelastic response of the VLBI antenna is approximately negligible, and due to this fact the primary and secondary reflectors of the structure can be considered as the main aerodynamic surfaces. They are approximated as flat plates, for use of the DLM to calculate the aerodynamic forces, due to the low aspect ratio of chord to thickness, being evaluated as $14 \%$ and $12.7 \%$ for the primary and secondary reflectors, respectively. Further details of the aeroelastic modelling are presented in the work of Nieto et al. [1].

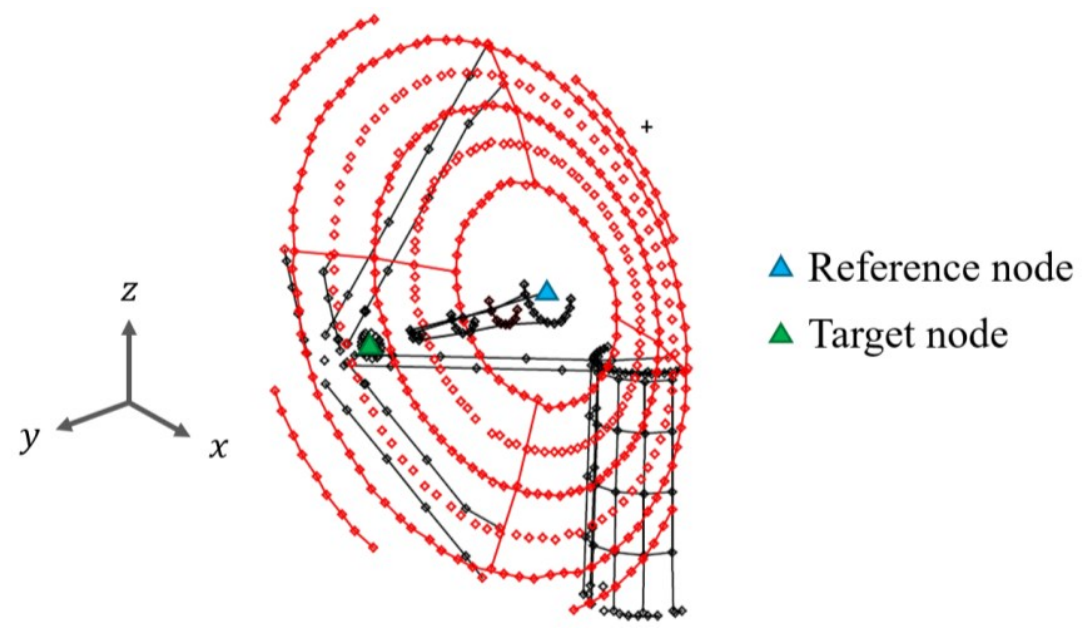

Figure 7.5: Reference and target monitoring grid points chosen for the VLBI antenna

Similarly to the Case Study I, as presented in the Section 7.2, the pointing accuracy of the antenna is assessed by means of a geometrical analysis considering the deviation angle of a reference point, which is located at an imaginary static point at the center of the primary reflector, with respect to a target point, which is defined at the center of the secondary reflector. The fixed distance between the two defined monitoring points is measured as $240.4 \mathrm{in}$, and is used throughout the analysis. Figure 7.5 illustrates the location of the referred grid points. 


\subsubsection{Operational cases gust profiles}

In terms of the aerodynamic excitations, fourteen operational cases are available for analysis of the aeroelastic response of the VLBI antenna structure, and are summarized in Table 7.2. Those operational conditions were provided by The National Aeronautics and Space Administration (NASA) and the industrial partner [38], and are based on requirements for effective operation of the antennas. Case 6 is defined as the primary operational scenario, and Case 14 the secondary.

Table 7.2: Operational cases for the VLBI antenna structure [38]

\begin{tabular}{ccccc}
\hline Case ID & $\begin{array}{c}\text { Disturbance speed } \\
\Delta v[\mathrm{in} / \mathrm{s}]\end{array}$ & $\begin{array}{c}\text { Mean wind speed } \\
v_{m}[\mathrm{in} / \mathrm{s}]\end{array}$ & Mach number & $\begin{array}{c}\text { Dynamic pressure } \\
{\left[\mathrm{lb} / \mathrm{in}^{3}\right]}\end{array}$ \\
\hline 1 & 34.15 & 109.36 & 0.008173 & 0.000555 \\
2 & 51.20 & 164.04 & 0.012259 & 0.001249 \\
3 & 68.20 & 218.72 & 0.016346 & 0.002221 \\
4 & 85.26 & 273.40 & 0.020432 & 0.003470 \\
5 & 102.30 & 328.08 & 0.024519 & 0.004997 \\
$6^{*}$ & 109.10 & 349.95 & 0.026153 & 0.005686 \\
7 & 119.30 & 382.76 & 0.028605 & 0.006802 \\
8 & 136.20 & 437.44 & 0.032692 & 0.008884 \\
9 & 170.20 & 546.80 & 0.040865 & 0.013881 \\
10 & 204.00 & 656.16 & 0.049038 & 0.019989 \\
11 & 237.90 & 765.52 & 0.057211 & 0.027207 \\
12 & 271.70 & 874.89 & 0.065384 & 0.035535 \\
13 & 305.50 & 984.25 & 0.073557 & 0.044974 \\
$14^{\dagger}$ & 339.20 & 1093.61 & 0.081730 & 0.055524 \\
\hline
\end{tabular}

Note: * Primary operational scenario, ${ }^{\dagger}$ Secondary operational scenario 
The disturbance speed and the mean wind speed given are utilized to compute the Davenport Spectrum and One-minus cosine gust excitation profiles, as previously presented in the Section 3.3.3, and are depicted in Figure 7.6. To model the profiles, the following parameters were used:

- For the One-minus cosine model, a gust scale length of $L_{g}=47,244.094$ in;

- For the Davenport Spectrum, a surface roughness of $z_{0}=11.811$ in, and same gust length.
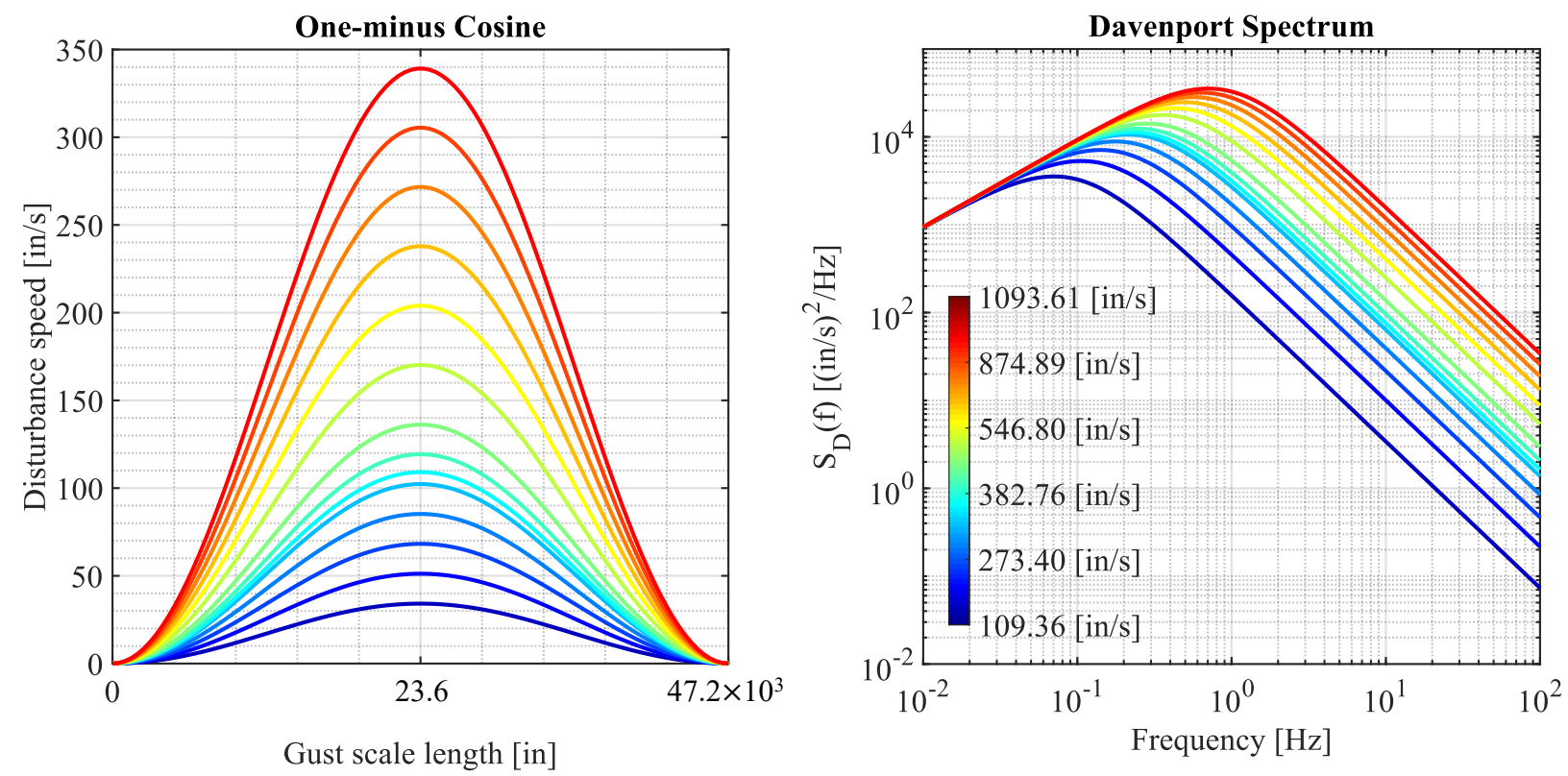

Figure 7.6: One-minus cosine and Davenport Spectrum gust models for the 14 operational cases analyzed

\subsubsection{Dynamic aeroelastic response analysis}

The PSD displacement function and the time-response displacement of the selected target node are given in Figure 7.7 and Figure 7.8, where the operational cases 6, 10 and 14 were chosen to illustrate the behavior of the structure under the dynamic excitations. The output of the rotational DOF was omitted from the results due to their negligible values when compared to the translational 
ones. Furthermore, the aeroelastic response to be presented herein is limited to the VLBI antenna modelled with a $0^{\circ}$ azimuth angle. For different azimuth angles, the mass and stiffness distribution of the antenna changes, which will likely result in distinct responses in the frequency and timedomains.
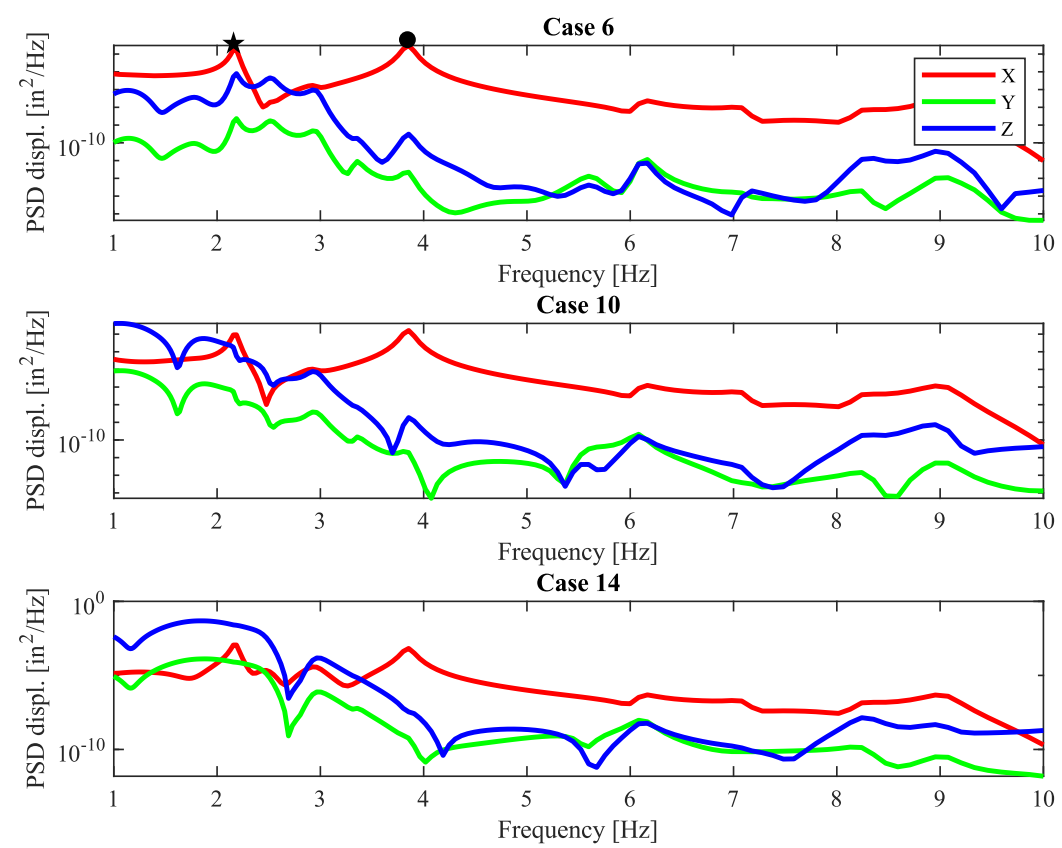

(a)
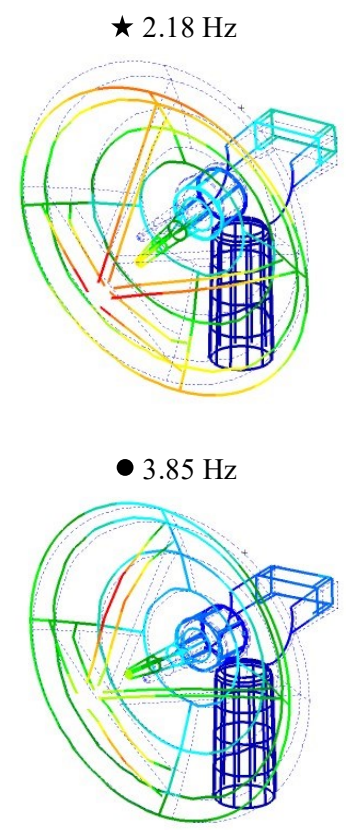

(b)

Figure 7.7: Uncontrolled structure response utilizing the PSD excitation modelled with DS.

(a) Target node PSD displacement functions of the operational scenarios considered. (b) Correspondent mode shapes for the first two resonance frequencies

In Figure 7.7, it is noticeable that the displacement in the $x$-direction dominates the response for the Case 6, due to its higher magnitude, when compared to the other DOF. This behavior is similar among the cases 1 to 10 , where the output value in the $x$-direction monotonically increases. The two resonance frequency peaks, evaluated at $2.18 \mathrm{~Hz}$ and $3.85 \mathrm{~Hz}$, represent mode shapes related to the global displacement of the primary collector and boom arms of the antenna structure. As it 
will be seen in the optimization procedure section, the displacement associated with those mode shapes are targeted to globally minimize the aeroelastic response. For the Cases 11 to 14 , a significant prominence of the $z$-direction response was observed. The reason for this behavior lies on the inertial component of the aeroelastic modelling of the system, where the disturbances in the $z$-direction are greatly magnified due to the actuation of the gravitational field in the rear counterweights and the center of mass of the antenna.
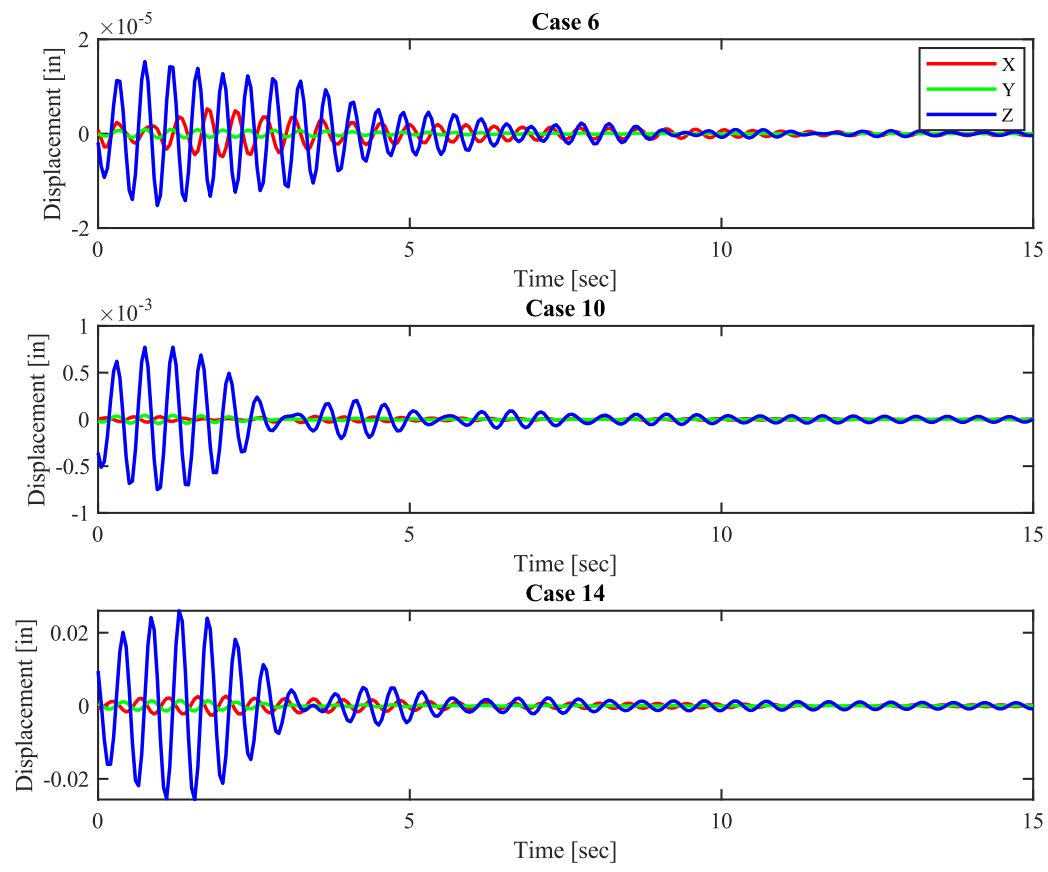

Figure 7.8: Target node time-response displacement of the operational scenarios considered for the InterTronic VLBI antenna, utilizing the TDG model

Now for the One-minus cosine gust excitation, as presented in Figure 7.8, the response was essentially dominated by the first global mode shape, identified at the frequency of $2.18 \mathrm{~Hz}$, in all fourteen cases. It is also important to mention that the response in the $z$-direction was magnified with the increase of the disturbance wind speed, due to the same reason as explained for the frequency domain analysis. Hence, the response mainly associated with the first mode shape 
should be targeted to minimize the aeroelastic response of the antenna, by means of the inclusion of the TMD control devices.

Finally, it is important to discuss one aspect of the numerical model developed herein. In terms of the modal characteristics, i.e. natural frequencies and mode shapes vectors, the ROM analyzed matches the high-fidelity DFEM provided by the industrial partner, therefore being accurate and precise in predicting the modal response of the structure. However, when dealing with the aerodynamic analyzes, approximations are necessary to be implemented, such as with the use of the DLM to estimate the aerodynamic properties of the antenna structure [1]. As previously mentioned, the DLM presents limitations in terms of its approximations due to the use of linearized potential equations, therefore not considering essential effects for the aerodynamic analysis, such as the geometry thickness of structures and large angles of attack [94]. The antenna model investigated has not yet been validated with higher-order computational fluid dynamic analysis or via experimental results, therefore the results estimated within this spectrum are taken as qualitative. The true level of error, while assumed to be small, will need to be properly validated to improve this framework.

In this way, Table 7.3 presents a comparison between the first five estimated global displacement modal frequencies of the DFEM, and the associated resonance frequencies obtained from the aeroelastic analysis of the new ROM. It can be seen that the low percentual error indicates good agreement between the values, especially regarding the first two frequencies, demonstrating that the model investigated is qualitatively correct. However, further refinement in the study must be considered, related to the model validation, in order to establish a good quantitative correspondence of the results from the aeroelastic analysis. This validation approach, nonetheless, 
is something out of scope from this thesis, and it is therefore suggested as a future work for further analysis refinement.

Table 7.3: Frequencies comparison between DFEM and the new ROM developed

\begin{tabular}{ccc}
\hline $\begin{array}{c}\text { Modal Frequency DFEM } \\
\text { SOL103 [Hz] }\end{array}$ & $\begin{array}{c}\text { Frequency ROM Gasparetto (2021) } \\
\text { SOL146 [Hz] }\end{array}$ & Error [\%] \\
\hline 2.214 & 2.188 & 1.2 \\
3.943 & 3.855 & 2.2 \\
6.491 & 6.166 & 5.0 \\
8.843 & 8.241 & 6.8 \\
9.109 & 8.432 & 7.4 \\
\hline
\end{tabular}

\subsubsection{Aeroelastic response fitness function definition}

The fitness functions utilized to minimize the aeroelastic response of the VLBI antenna are similar to the ones presented in the Case Study I, but with some specific differences. The first noticeable distinction is the implementation of multiple cases to be analyzed within a single iteration. By doing this, several cases can be defined as objectives to be minimized for the same set of design variables. This improvement brings some advantages, specially related to finding an optimal solution that can satisfactorily attenuate the response for a great variety of aerodynamic disturbance profiles. The main drawback with this strategy revolves in the computational capacity available for the simulations, due to the fact that multiple instances of MSC NASTRAN ${ }^{\circledR}$ have to be executed at the same time to evaluate the individuals, which can result in increase in the computation time of each iteration. 
Regarding the formulation of the fitness function related to the aeroelastic response, for the frequency domain analysis, it is used the maximum peak of the PSD displacement function, taken among all DOF considered. The time-domain calculation of the fitness function remains unchanged as previously presented, therefore being the area under the $U_{R S S Q} \times t$ curve. Equations (7.3.a) and (7.3.b) describe the fitness function implemented,

PSD:

$$
\left(U_{\text {fitness }}\right)_{j}=\max \left\{S_{x-j}\left(\omega_{\text {crit }}\right), S_{y-j}\left(\omega_{\text {crit }}\right), S_{z-j}\left(\omega_{\text {crit }}\right)\right\} \text {, }
$$

$$
j=1,2, \ldots, N_{\text {case }}
$$

TDG:

$$
\left(U_{\text {fitness }}\right)_{j}=\int_{0}^{t_{f}} U_{R S S Q-j} d t
$$

$$
U_{R S S Q-j}(t)=\sqrt{U_{x-j}^{2}(t)+U_{y-j}^{2}(t)+U_{z-j}^{2}(t)}
$$

where $U_{\text {fitness }}$ is the fitness function to be minimized for both frequency and time domain analysis, $\left\{S_{x-j}, S_{y-j}, S_{z-j}\right\}$ are the PSD displacement functions obtained for each DOF, $\omega_{\text {crit }}$ is the associated critical frequency, and $N_{\text {case }}$ is the number of operational cases considered. The variable $U_{R S S Q}$ is the RSSQ output from the displacements in the time domain $\left\{U_{x}, U_{y}, U_{z}\right\}$. It is important to mention that the minimization of the total included mass of the TMD remained as part of the multi-objective functions for both frequency and time domain simulations.

In order to summarize the $U_{\text {fitness }}$ values for the uncontrolled structure under each operational case, considering the two gust excitation profiles, Figure 7.9 is presented. It is possible to observe a trend of increase in their values with the increase of the wind speed of the correspondent 
operational case. The values presented herein are utilized for a percentual comparison of pointing error reduction after the inclusion of the optimized TMD set.
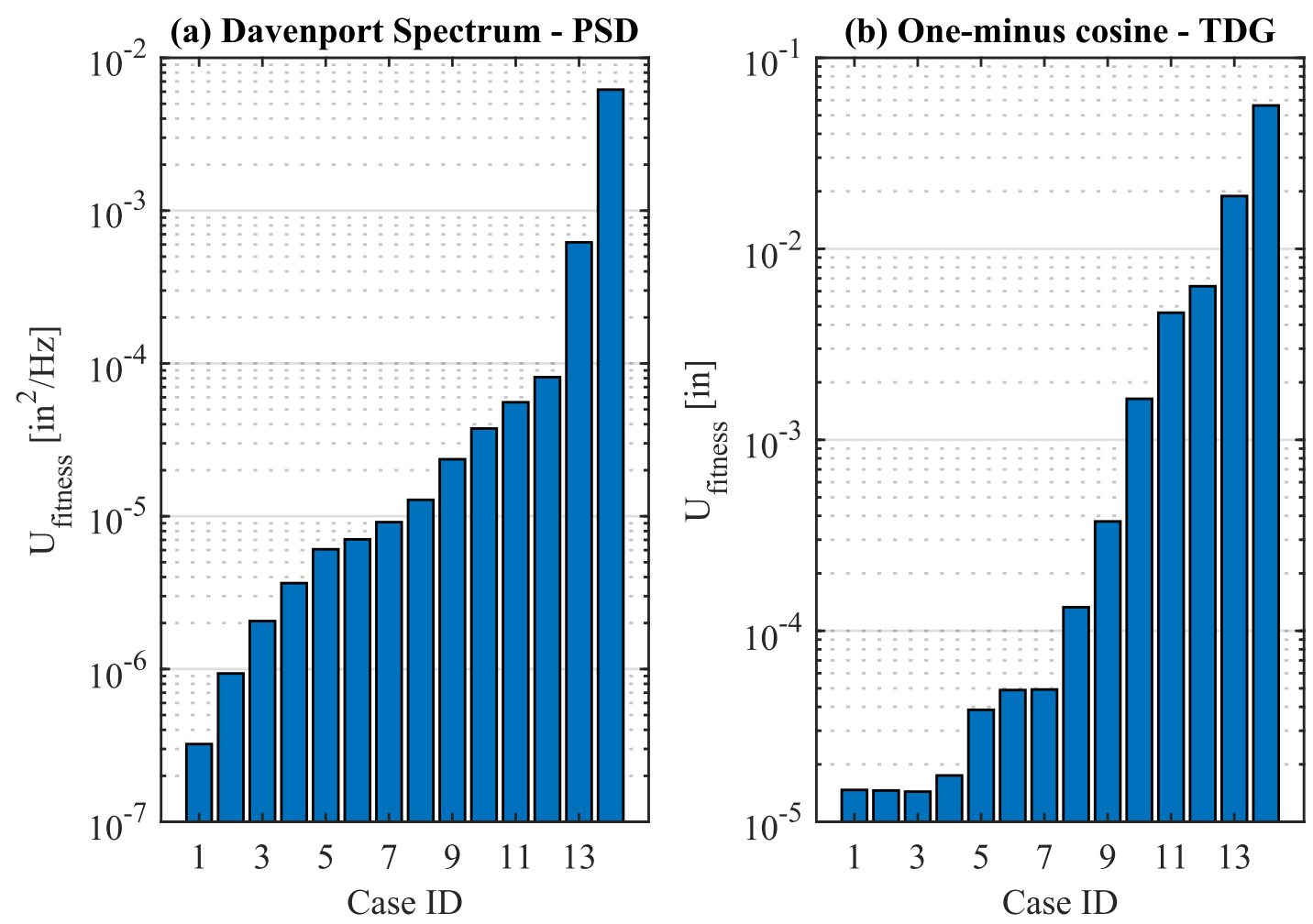

Figure 7.9: Fitness function evaluated for the uncontrolled structure under the (a) PSD excitation modelled with the DS and (b) One-minus cosine gust models

\subsection{Response attenuation using optimized single-TMD}

One configuration of TMD is investigated to minimize the aeroelastic response of the VLBI antenna structure, being based on the inclusion of a single-TMD in the secondary reflector, specifically at the assigned target node. This configuration brings the advantage of simplicity of implementation in the real structure, due to the fact that only one TMD is necessary to be attached 
into the boom arms. With this strategy, a single MDOF resonator is assigned to the structure, and can be tuned accordingly to the resonance frequencies identified in the previous sections.

The mass inclusion in the VLBI antenna structure again is a critical design parameter of the analysis, especially because the TMD need to be proportional to the entire system's mass. For the case Study I, a high percentage mass inclusion was utilized within the simulations in order to effectively test the full capabilities of the method implemented. On the other hand, the VLBI antenna is a civil structure, due to its dimensions and weight, and a substantially more complex model, for example due to the inclusion of counterweights, multiple materials, and different FE composition, thus it is a fact that a high percentage of mass inclusion, such as in the Case Study I, could result in potential structural issues of static load deformation or fatigue of the slender boom arms truss. Notwithstanding, the concurrent analysis of internal forces and stresses in the elements is a topic out of scope of the thesis.

However, to execute the analysis with realistic inputs, a limit of 5\% of mass inclusion due to TMD placement is adopted herein. As it will be shown in the further sections, even with very low mass inclusions it is possible to characterize a significant attenuation in the structure's response when the other design parameters are correctly tuned. Therefore, in future works, it is of interest to implement external routines to evaluate the mechanical stress, or even to treat this parameter as a design variable for the optimization problem, selecting design parameters of the TMD that would guarantee safety in their use.

In terms of the settings of the MOGA procedure, the following parameters were used in the simulation of the single-TMD:

- Crossover fraction: $80 \%$; 
- Elitism probability: $2 \%$;

- Migration factor: $20 \%$;

- Migration interval: 20;

- Pareto fraction: $35 \%$.

Moreover, the stopping criteria for the optimization procedure utilized is the same as discussed in the Case Study I, being the chosen maximum number of generations, or the average change in the spread of the Pareto front. For the single-TMD configuration, only three design variables are necessary to run the simulation, being the mass, stiffness and damping coefficient of the TMD, as previously specified. To obtain the effective convergence of the results, a population size of 100 and a maximum number of generations of 30 are utilized. With those values, a total of 3000 analyzes are carried with the MOGA optimization procedure.

The estimative of the bounds for the design variables are based on the formulation presented in Warburton [134], where it is considered a random load, such as the aerodynamic gust excitation, and the two target frequencies that represent the peaks of resonance, as shown in Figure 7.7. The bounds are found based on the value of the mass inclusion ratio, which assumed a range between $0 \%$ and $5 \%$. The values utilized in the simulations are described in the Table 7.4.

The results for the optimization framework are now presented, considering the operational cases and both models of atmospheric disturbances. The results are given in terms of Pareto fronts, output response functions for the target node, and charts that indicates the optimum values of the single-TMD found. 
Table 7.4: Design variables bounds for the single-TMD analysis

\begin{tabular}{cccc}
\hline Bounds & $m_{T M D}[\mathrm{lb}]$ & $c_{T M D}[\mathrm{lbf.s} / \mathrm{in}]$ & $k_{T M D}[\mathrm{lbf} / \mathrm{in}]$ \\
\hline Lower (min.) & 77.2 & 0.30 & 228.40 \\
Upper (max.) & 3861.0 & 571.0 & 17130.0 \\
\hline
\end{tabular}

\subsubsection{Frequency domain analysis with the PSD modelled with the Davenport Spectrum}

The optimization procedure is implemented for the excitation modelled with the PSD function given by the DS model. It is important to notice that all 14 operational cases were concurrently evaluated in the analysis, and the Pareto front achieved is the result of the individuals that mostly minimized the aeroelastic response of the antenna considering all the excitations. Hence, in the graphs that follows, not necessarily an individual that achieved a good response attenuation for one operational case will be valid for others, and vice-versa, causing the Pareto front to assume a discontinuous behavior for some operational cases. However, the Pareto front individuals are the ones that concurrently minimize all cases. This observation is also valid for the time domain analysis. 

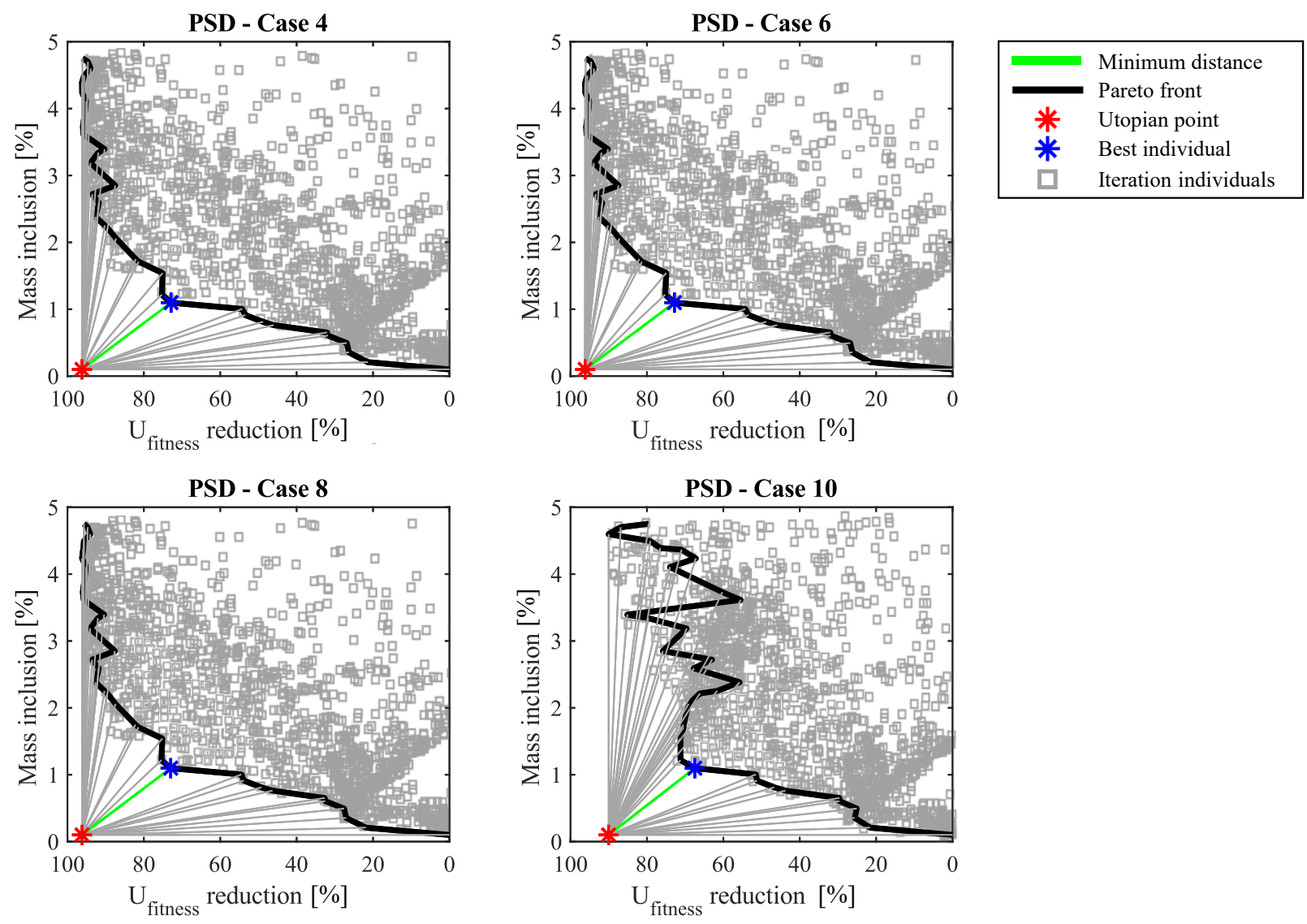

Figure 7.10: Multi-objective Pareto front of all individuals analyzed, indicating the utopian point and the best individual using the single-TMD for the operational cases $4,6,8$ and 10 modelled with the DS model

Representatively, an example of output from the MOGA framework is shown in Figure 7.10, where the pointing error and mass inclusion are minimized for the operational cases 4, 6, 8 and 10 . In the Figure 7.10 it is possible to observe the expected behavior of decrease in the aeroelastic deviation associated with a direct increase in the mass inclusion in the antenna, therefore establishing a trade-off problem. The utopian point indicated encompasses the extremes of the Pareto front, hence considering all individuals obtained at the convergence of the optimization 
algorithm. The selection of the utopian point followed the unitarily weighted Euclidean Metric as previously described. In addition, the percentual reduction presented in Figure 7.10 is given by Equation (7.4),

$$
U_{\text {fitness }} \text { reduction [\%] }=\left|\frac{U_{\text {fitness }, U N C}-U_{\text {fitness }, T M D}}{U_{\text {fitness }, U N C}}\right| \times 100
$$

where $U_{\text {fitness,UNC }}$ and $U_{\text {fitness,TMD }}$ are the net aeroelastic response for the uncontrolled structure and with the attached single-TMD, respectively.

The PSD displacement response functions, for the target node, of the Pareto front individuals are presented in Figure 7.11 for each respective DOF considered in the study. The first noticeable aspect is that the inclusion of the single-TMD is capable of attenuating the response in all directions, similarly to the observed behavior for the multiple-TMD configuration in the Case Study I. Moreover, with the optimized constitutive parameters of the TMD, it is possible to characterize an average of $90 \%$ reduction in the aeroelastic response, with a maximum mass inclusion of 5\%, thus demonstrating the excellent effectiveness of this method.

However, as explained before, the mass addition is a factor that can potentially impact the structure stability in operation, therefore it is interesting to select the individual with the best trade-off between mass inclusion and aeroelastic response attenuation. The best individual found based on the utopian point is listed in the Appendix B.1 of the thesis, alongside with all other individuals obtained from the Pareto front. The parameters presented are the same for all DOF considered, i.e., directions $x, y$ and $z$ assume the same properties of mass, damping coefficient and stiffness. 

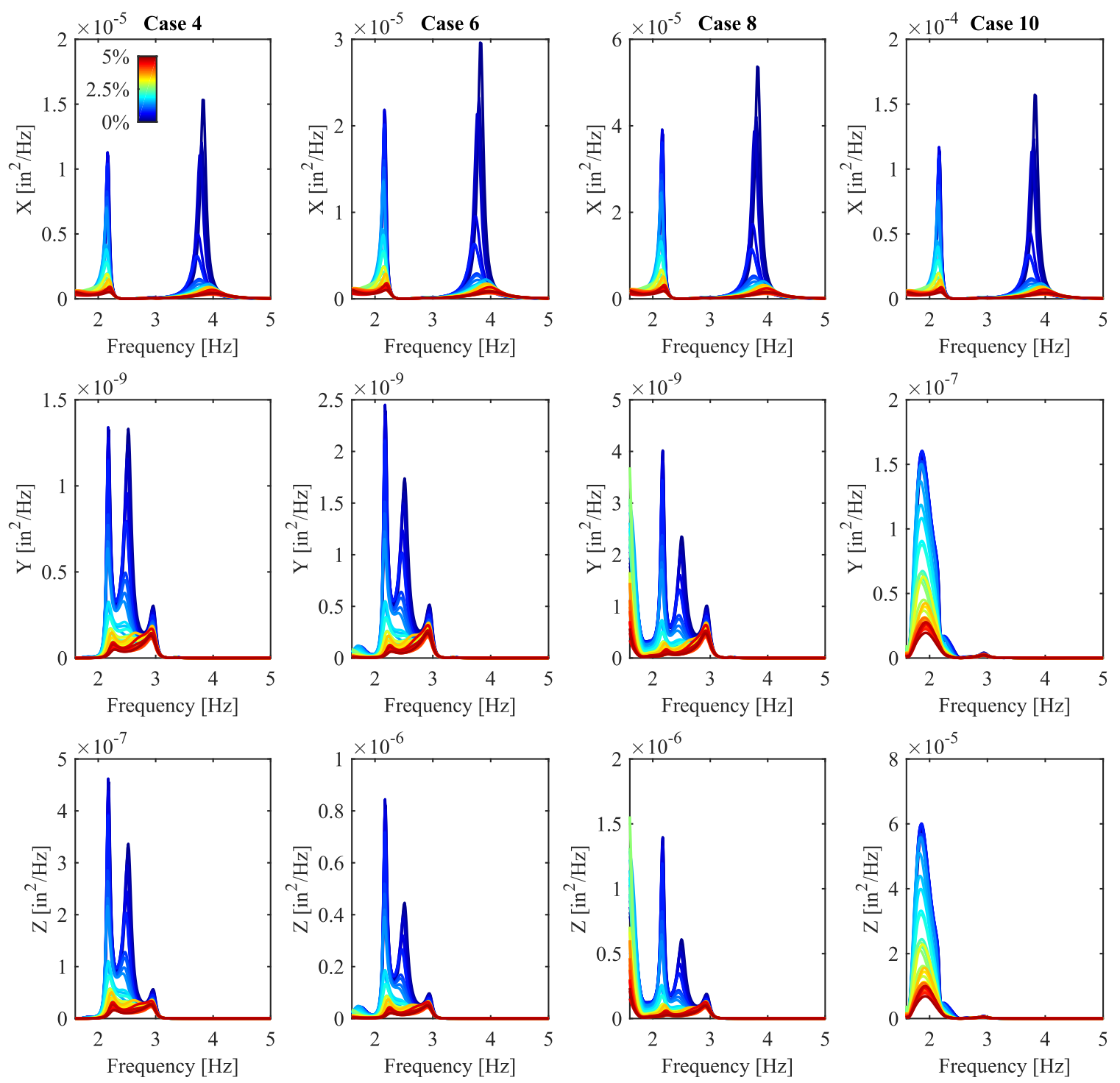

Figure 7.11: Concurrent aeroelastic response minimization using the single-TMD configuration for the operational cases 4, 6, 8 and 10 modelled with the DS. The PSD displacement functions of the target node are presented for each respective direction, where the color bar indicated represents the percentage of mass inclusion in the antenna

Figure 7.12 is introduced in order to summarize the results obtained for the aeroelastic attenuation of all cases, using the best individual as listed in the Appendix B.1. A comparison between the uncontrolled and controlled structure with the single-TMD configuration is depicted in the referred 
figure. As a major observation, for all operational scenarios it is possible to characterize a reduction in the aeroelastic response, with greatest efficiency comprehending the cases 1 to 12 .

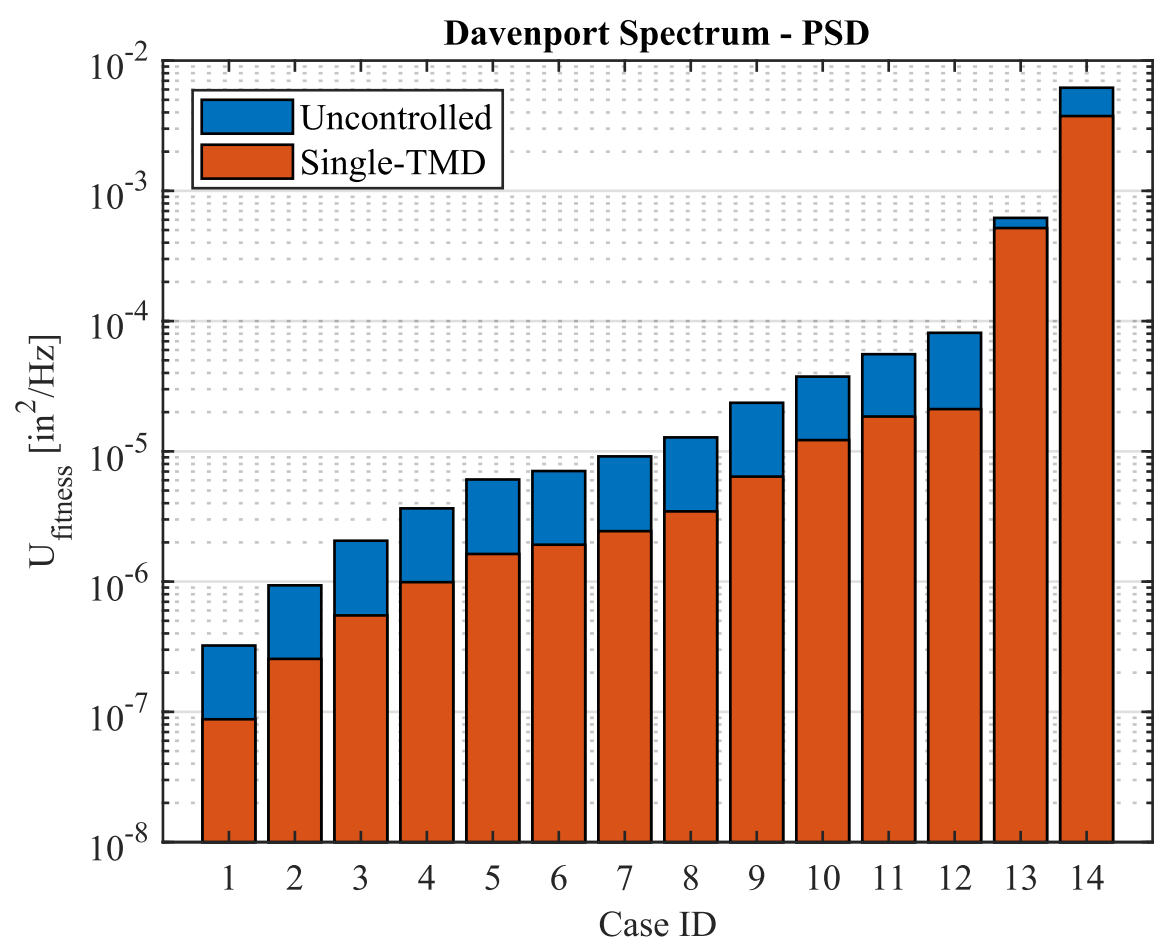

Figure 7.12: Aeroelastic response fitness function comparison between the uncontrolled and the controlled structure with an optimal single-TMD. Results presented for the Davenport Spectrum model

It can also be seen in Figure 7.12 that the attenuation efficiency was significantly reduced for the cases 13 and 14. This behavior is attributed to the fact that for those two last cases, the response is essentially dominated by the $z$-direction, differently from the other cases which presented either a dominance of the $x$-direction or an equivalence in magnitude of the $x$ - and $z$-direction. The singleTMD control device presents a limitation in terms of this characteristic, since their design is essentially dependent on a target frequency and the DOF response magnitude [42]. Therefore, this reduction of the response attenuation efficiency is expected when the same optimized TMD 
parameters are used to concurrently analyze multiple cases. Nevertheless, the pointing error was effectively reduced for all cases, and in specific, for the primary and secondary operational cases it was possible to characterize a reduction of $73.2 \%$ and $39.4 \%$, with a structural mass inclusion of approximately $1 \%$.

\subsubsection{Time domain analysis with the One-minus cosine gust model}

Utilizing now the One-minus cosine gust model as the excitation profile applied to the VLBI antenna, the performance of the inclusion of an optimized single-TMD is investigated. Again, all 14 operational scenarios are concurrently analyzed, such that one specific configuration of design variables is capable of minimizing the aeroelastic response for multiple cases. As shown in Equation (7.3.b), the objective function related to the aeroelastic response is taken as the area underneath the graph of the net displacement of the DOF over time. This way, a lower area indicates that the signal is being attenuated faster than a case with a higher graph area value.

Similarly as before, all 14 operational cases were concurrently evaluated in the analysis, and the Pareto front achieved is the result of the individuals that mostly minimized the aeroelastic response of the antenna considering all the excitations. A representative output from the framework is presented in Figure 7.13 and Figure 7.14, where the concurrent minimization of mass inclusion and area underneath the curve of pointing error with respect to time, displaying the operational cases 4, 6, 8 and 10. In Figure 7.13, the Pareto fronts obtained indicate a similar trend of increase of mass inclusion when decreasing the aeroelastic response at the selected target node. Furthermore, similarly to the frequency response analysis, it is possible to characterize an average of $85 \%$ reduction in the aeroelastic response, with a maximum mass inclusion of $3 \%$, thus 
demonstrating the excellent effectiveness of this method for attenuating excitation profiles with impulse-like nature, such as the One-minus cosine gust model.
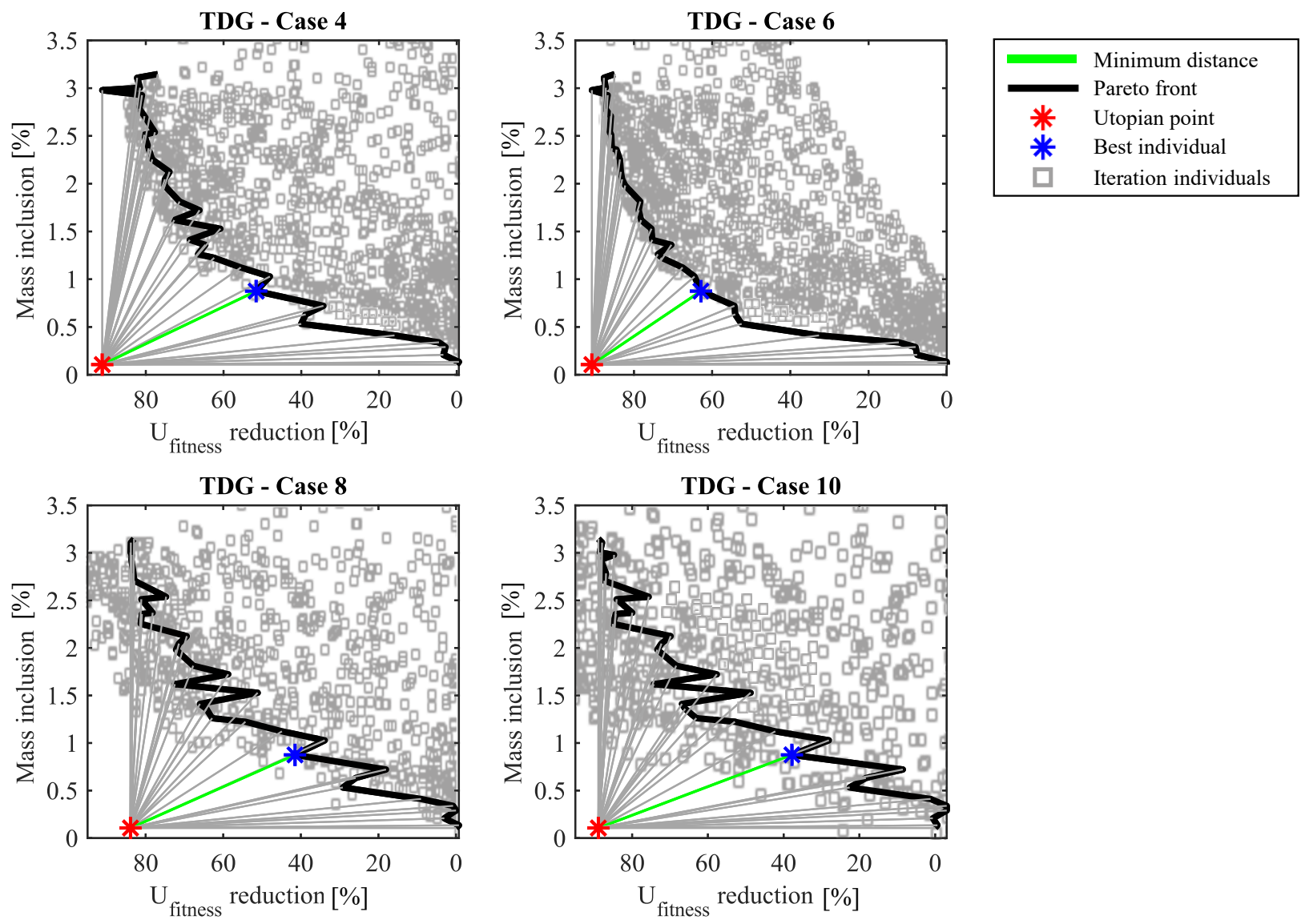

Figure 7.13: Multi-objective Pareto front of all individuals analyzed, indicating the utopian point and the best individual using the single-TMD for the operational cases 4, 6, 8 and 10 modelled with the One-minus cosine gust model

Differently from the previous analysis, for mass inclusions greater than $3 \%$ it is generally observed a decrease in the attenuation efficiency for the operational cases, therefore the Pareto fronts were only characterized up until the referred percentage. This behavior is associated with the impulse- 
like nature of the excitation possibly introducing an instability phenomenon due the higher values of mass inclusion, thus reducing the effectiveness of actuation of the single-TMD.
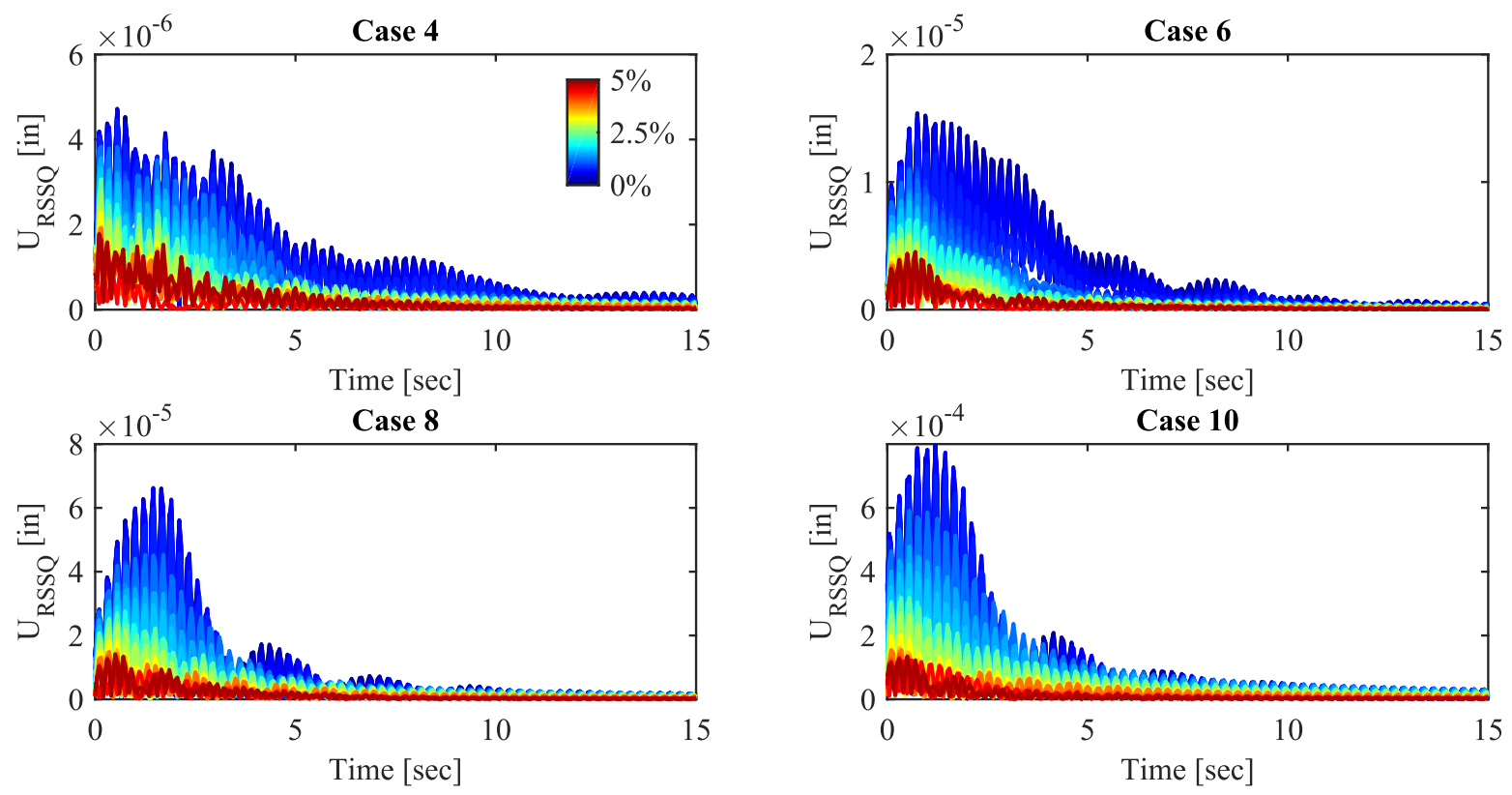

Figure 7.14: Concurrent aeroelastic response minimization using the single-TMD for the operational cases under the One-minus cosine gust model. Time response functions of the target node displacement are presented as a RSSQ of each DOF, where the color bar indicated represents the percentage of mass inclusion in the antenna

Figure 7.14 depicts the time-domain response of the target node for the Pareto front individuals for the operational cases $4,6,8$ and 10 , where the mass inclusion presents an increase from $0 \%$ to $3 \%$. The net deviation is indicated in the graph, therefore being the RSSQ of the displacement for each DOF. When the TMD mass is near $0 \%$, the response is similar to the uncontrolled structure and the oscillations amplitudes are still observable until around 7.5 seconds after the excitation from the One-minus cosine gust. With the attachment of the optimized TMD, and with higher mass inclusion percentages, the signal is considerably attenuated, being damped faster, and the 
maximum amplitude experienced by the target node reduced. This characteristic indicates that the pointing accuracy can be successfully maintained when the antenna structure is subjected to impulse-like excitations. In similar fashion, the best individual found based on the utopian point method is listed in the Appendix B.2 of the thesis, alongside with all other individuals obtained from the Pareto front. The parameters presented are the same for all DOF considered, i.e., directions $x, y$ and $z$ assume the same properties of mass, damping coefficient and stiffness.

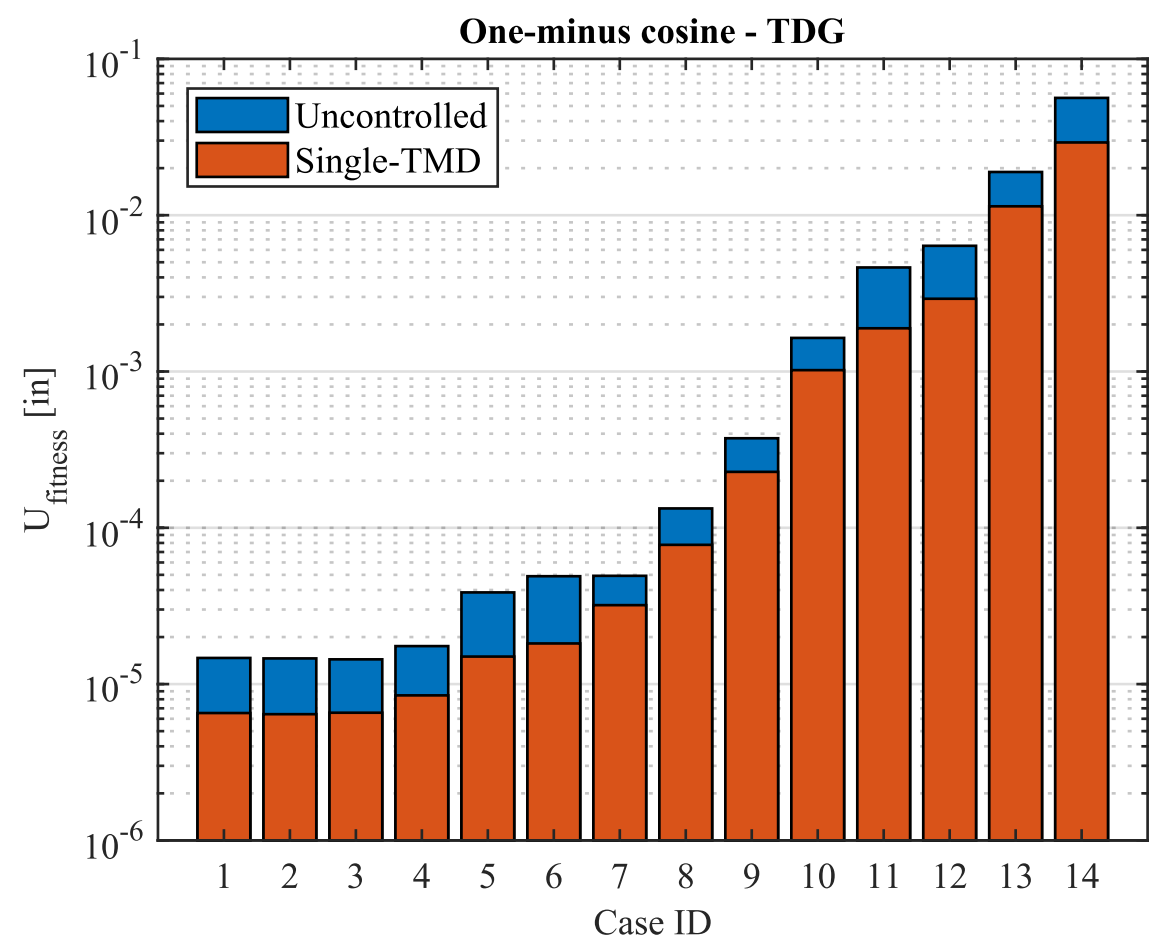

Figure 7.15: Aeroelastic response fitness function comparison between the uncontrolled and the controlled structure with an optimal single-TMD. Results presented for the One-minus cosine gust model

The performance of the best individual identified is compared with the uncontrolled structure, for all operational cases, in Figure 7.15. Again, for all excitations investigated it is possible to characterize a reduction in the aeroelastic response by including the optimized single-TMD. The 
lower mean wind speed cases, comprehending the cases 1 to 6 , presented the highest reduction in the aeroelastic response of the target node between 51 and $63 \%$. For cases with higher mean wind speed the attenuation percentage is lower, as expected, except for the cases 11 and 12 which presented an increase in the attenuation effectiveness.

\subsubsection{Comparison of the aeroelastic response fitness for the gust models}

As per the results presented in the previous section, both analysis in the frequency-domain and time-domain presented different values for the optimal TMD. This is expected to occur since the excitation models are different from each other, and the constitutive parameters of the TMD are dependent upon that matter. However, since the solution to be implemented in a VLBI antenna system must be unique, it is necessary to select one optimal design variables set and test among each gust excitation profile, and thus evaluate the performance of the auxiliary mass. There are two ways of executing this analysis:

1. The first is the most formal and accurate, which would be to concurrently run the optimization framework considering all 14 operational cases for both gust models. This way, it would be possible to determine a set of design variables that globally minimizes the aeroelastic response of the VLBI antenna. The drawback of this method lies on the computational capacity limitation to run the framework, which would exponentially increase the computation time to evaluate the fitness functions, making it impractical to obtain results.

2. The second is an approximation approach, and it is related to test the permutation of best individuals previously found using the optimization framework independently for each gust excitation profile. For example, taking the optimal set of design variables from the 
frequency-domain analysis and test it within the time-domain analysis, quantifying the performance, and vice-versa. This way, the best individual can be selected based on this proposed criterion. The drawback of this approach lies on the fact that not necessarily the optimal solution for one model will attenuate the other one, thus existing the possibility of the response being magnified by the inclusion of the single-TMD.

For this study, the second approach is selected, such that the best solution can be found from the data set presented in the work. To do so, the following individuals presented in Table 7.5, identified as the best by means of the utopian point method as described in the Appendix B.1 and B.2, are used in order to find the unique solution.

Table 7.5: Best TMD individuals for the PSD and TDG excitation models

\begin{tabular}{ccccc}
\hline Configuration ID & Gust model & $\mathrm{m}(\mathrm{lb})$ & $\mathrm{c}(\mathrm{lbf} . \mathrm{s} / \mathrm{in})$ & $\mathrm{k}(\mathrm{lbf} / \mathrm{in})$ \\
\hline A & PSD & $8.48 \mathrm{E}+02$ & $1.67 \mathrm{E}+01$ & $5.04 \mathrm{E}+02$ \\
B & TDG & $6.76 \mathrm{E}+02$ & $6.07 \mathrm{E}+00$ & $2.93 \mathrm{E}+02$ \\
\hline
\end{tabular}

Figure 7.16 depicts the results for the comparison of the aeroelastic response attenuated by the inclusion of the optimal TMD associated for each gust model, where configuration A is indicated by the blue line, and configuration B by the red line. As it can be seen, the parameters found for the optimal TMD under configuration B presented the overall best results for minimizing the aeroelastic response in both gust models. For the PSD excitation in Figure 7.16(a), both optimal TMD resulted in practically the same level of attenuation from the operational cases 1 to 12 , however, for the last two cases, the configuration B demonstrated to be superior in attenuating the aeroelastic response when compared to configuration A. As for the TDG excitation in Figure 
7.16(b), the configuration B again displayed a slightly better performance set side by side for all cases with configuration A. Hence, the results indicate that a single-TMD with constitutive parameters evaluated as in the configuration B could result in better attenuation performance of the aeroelastic response of the VLBI antenna structure for both of the gust excitation models.

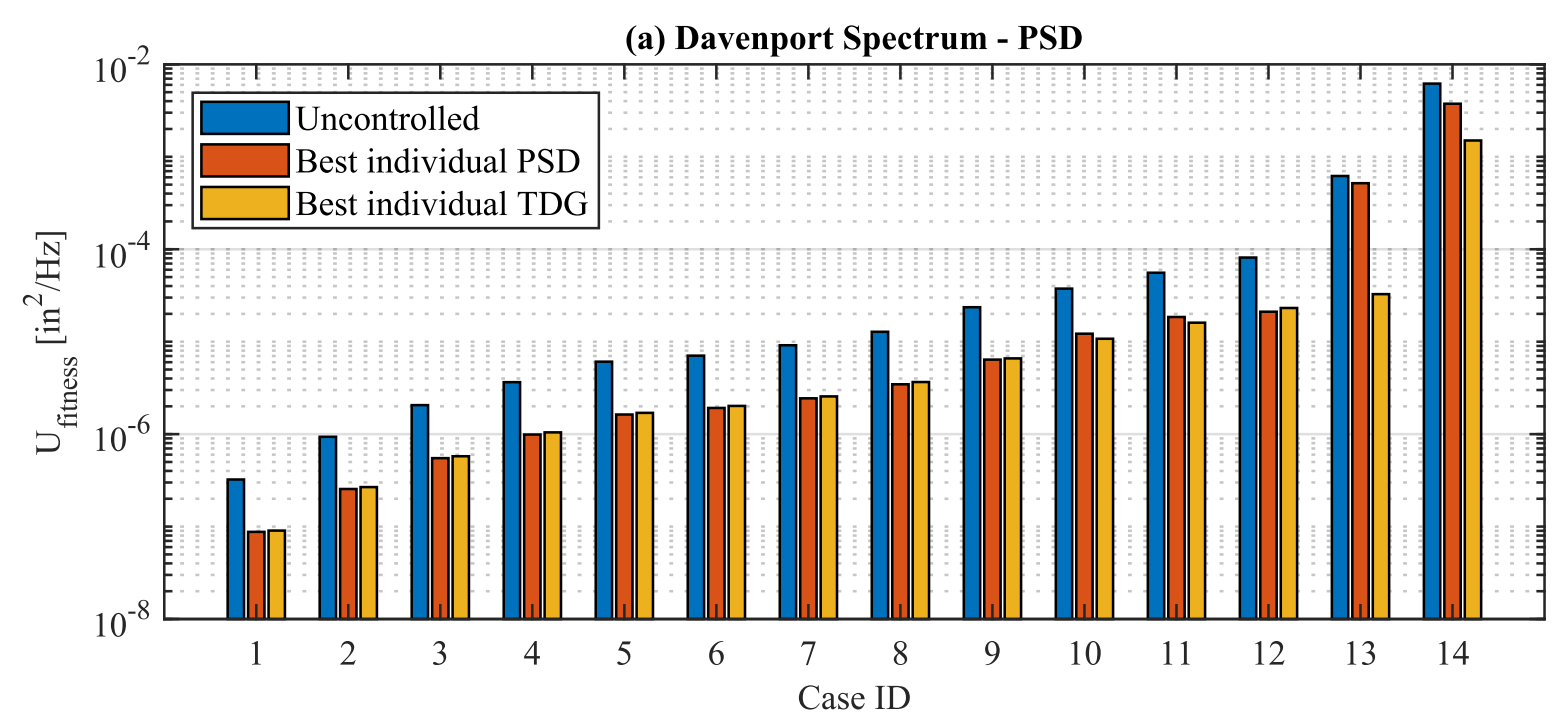

(b) One-minus cosine - TDG

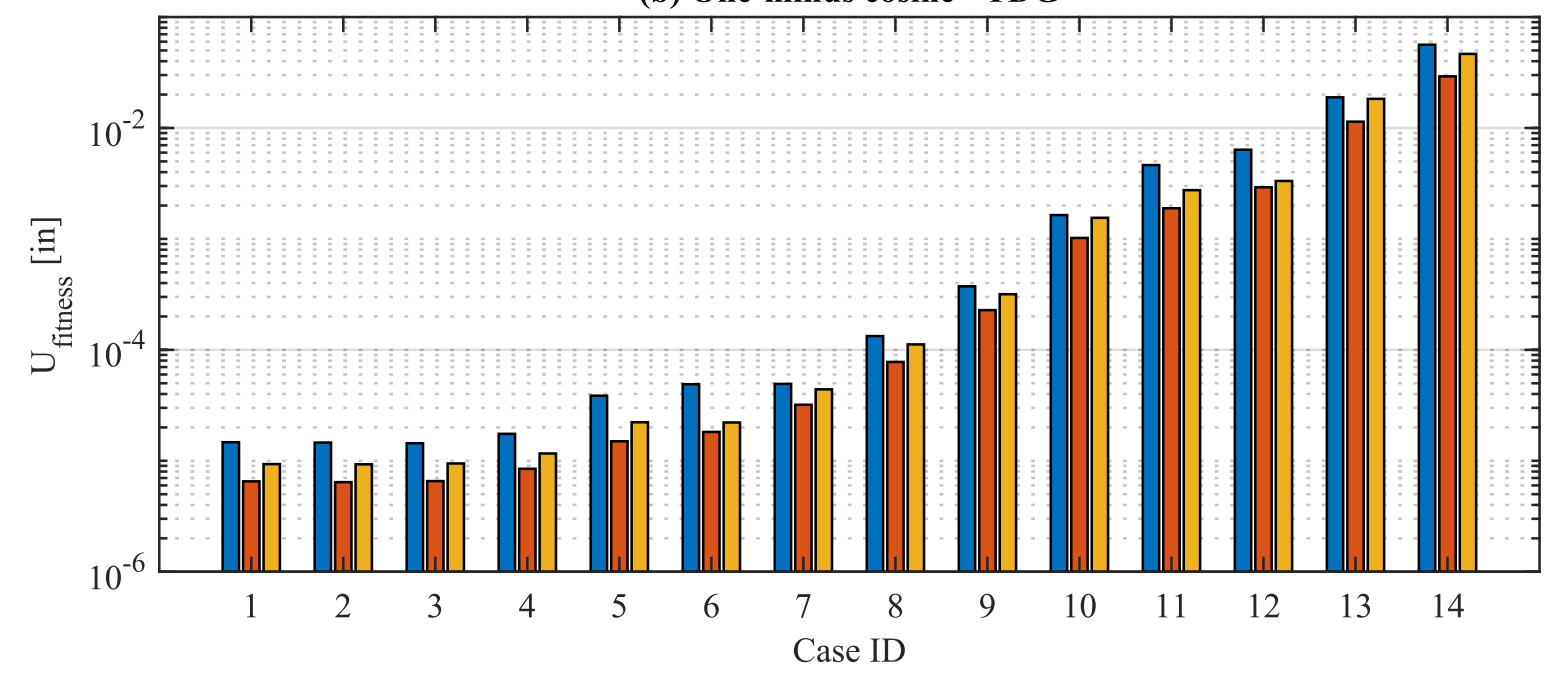

Figure 7.16: Comparison of the aeroelastic response fitness for the (a) PSD and (b) TDG gust models. The best individuals found from the optimization framework are evaluated among both gust models 


\subsubsection{Factorial analysis of TMD design variables with a defined mass inclusion}

In order to further understand how the change of the design variables affects the aeroelastic response of the target node, a full factorial analysis is conducted. This is an important set of results due to the fact that it should inform the designer the effect of changing the design variables of the TMD, and how they influence the final response. The Pareto front optimal solution is unique, however, there are other sets of design variables that provide similar performance to attenuate the aeroelastic response, hence being characterized as feasible solutions for the problem.

To do so, the operational case 6 is selected to representatively investigate the performance of the TMD, for both DS and One-minus cosine gust models. The mass inclusion, taken as constant in this factorial analysis, is defined as the optimal solution found in the previous section, such that $m=848 \mathrm{lb}$. As for the damping coefficient and stiffness, the ranges utilized are $0.30 \leq c_{T M D} \leq$ 60 [lbf. s/in], and $220 \leq k_{T M D} \leq 1700$ [lbf/in], respectively.

Figure 7.17 depicts the contour surfaces of the factorial analysis, for each gust excitation model. As it can be seen from the design space, the global maximum percentage reduction is present at the bottom left portion of both gust excitation models, for the operational case 6 . These values obtained with the factorial analysis are similar to those found with the MOGA procedure, after selecting the best individual with the utopian point method, hence demonstrating that the framework is efficient in finding the best individual among a large design space. 


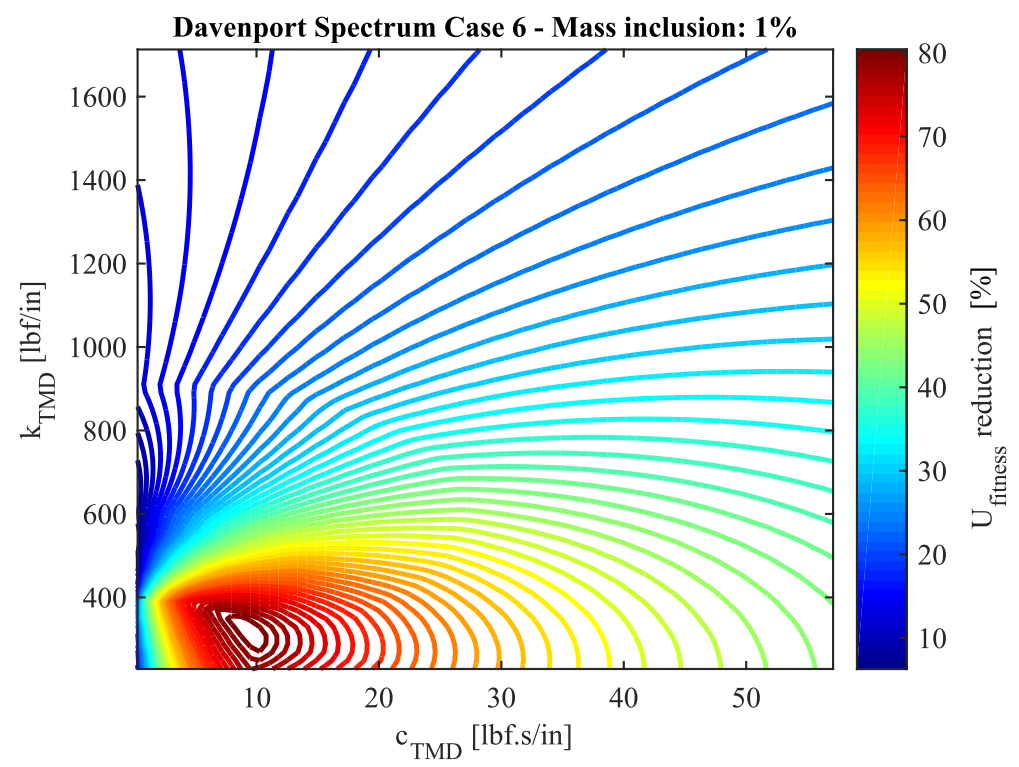

(a)

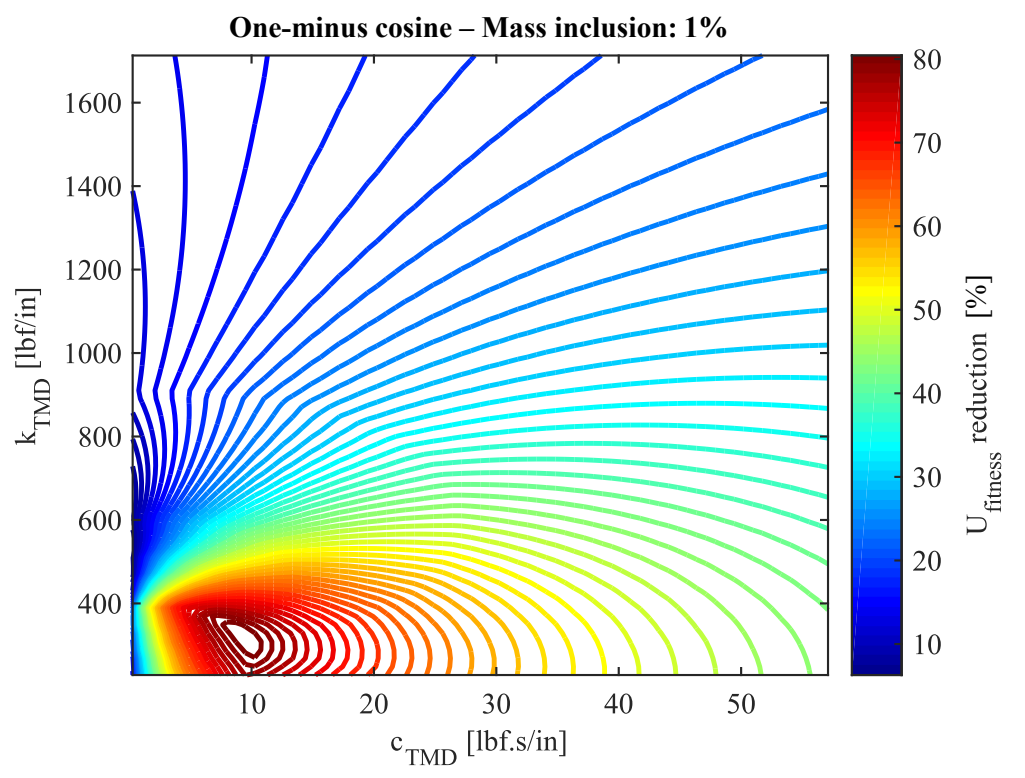

(b)

Figure 7.17: Factorial analysis of design variables considering a constant mass inclusion, for the operational case 6. (a) Davenport Spectrum and (b) One-minus cosine gust models 


\section{Chapter 8: Conclusions and future work}

\subsection{Conclusions}

In this thesis, an optimization framework to concurrently attenuate the dynamic aeroelastic response and minimize mass inclusion of TMD applied to aerospace structures was proposed, by means of the use of TMD, a type of passive control devices. The algorithm integrates in-house developed routines in both MATLAB ${ }^{\circledR}$ and MSC NASTRAN ${ }^{\circledR}$, using the dynamic aeroelastic solution 146 and an elitist MOGA to determine the optimal parameters of the control devices to minimize the objective functions considering different models of atmospheric disturbance excitations, in both frequency and time domains. One of the main characteristics of the developed framework is that it can be essentially implemented for any structure or boundary condition modelled within MSC NASTRAN ${ }^{\circ}$ environment, therefore being extremely versatile and applicable to other fields of engineering than aerospace structures. The concluding remarks of the various analyses performed are summarized below:

Case study I - Aeroelastic response attenuation of a simplified Earth-based radio antenna model

In this chapter, a FE model of a large Earth-based antenna was created, where its aeroelastic response was assessed in terms of the pointing accuracy. Furthermore, the use of multiple-TMD configuration has not yet been investigated in the literature applied to this type of structure, hence consolidating one important novelty of the work. Given the evaluation of the mode shapes in the peak frequency response, three different configurations of multiple-TMD placement were investigated and compared. From the optimization results, best individuals were determined 
according to the implementation of the utopian point method considering the weighted Euclidean metric, for different operational load cases.

It was found that, for both atmospheric disturbance models, the overall best individual was given by the Configuration 1, which presents the multiple-TMD placed in the backside truss of the antenna. This was an expected result due to the fact that the placement of multiple-TMD is more effective in regions of higher displacements, therefore providing better vibration attenuation capabilities. By utilizing the multiple-TMD under Configuration 1, it was possible to characterize a reduction in the pointing error of the antenna by $62.0 \%$ and $51.6 \%$ for the primary and secondary operational cases considering the PSD excitation using the DS model, respectively, and $39.2 \%$ and $36.8 \%$ for the primary and secondary operational cases considering the One-minus cosine gust model, respectively, hence demonstrating the excellent performance of the implementation of such passive control method.

Therefore, this case study contributed to propose advanced and useful tools to determine the optimal parameters of multiple-TMD passive control devices under complex loadings scenarios, as a primordial step towards the use of such systems in applications that commonly employ active or semi-active solutions, which are of sophisticated management and calibration.

\section{Case study II - Pointing error reduction of a $12 \mathrm{~m}$ Earth-based radio VLBI antenna model}

This chapter investigated the aeroelastic response attenuation by using a single-TMD configuration, applied to a FE model of a $12 \mathrm{~m}$ Earth-based radio VLBI antenna, designed by InterTronic Solutions. Due to the large number of DOF associated with the DFEM, provided by the manufacturer, the model had to be reduced in order to achieve results within a reasonable time. In the Nieto et al. [1], a method to reduce the order of the FE model was introduced, making it 
possible to run the solution 146 in MSC NASTRAN® within a matter of few minutes. However, to use the proposed ROM in an optimization procedure, a further refinement of the order reduction was necessary to be implemented. Within this scope, all DOF that were not associated with boundary conditions, aerodynamic panels, structural spline, or the truss boom arms of the reflectors, were eliminated from the analysis by employing the superelement method in MSC NASTRAN®. This allowed to reduce each iteration time of the solution 146 by $67 \%$ while still achieving excellent correlation measured with the MAC, and low error between the natural frequencies, when benchmarked with the model presented by Nieto et al. [1].

The aeroelastic response of the VLBI antenna indicated two frequencies that are of critical investigation, being $2.18 \mathrm{~Hz}$ and $3.85 \mathrm{~Hz}$, representing the mode shapes related to the global displacement of the primary collector and boom arms of the antenna structure. Furthermore, with the increase of the mean wind speed of the operational cases, a prominence of the $z$-direction response of the target node at the antenna was observed.

In order to determine the optimal single-TMD constitutive parameters to minimize the aeroelastic response of the antenna, an innovative approach was implemented, such that all operational cases were concurrently investigated utilizing the MOGA framework proposed. This way, the best individual found would attenuate the response for all cases. Similarly to the Case Study I, the best individual was found by means of the utopian point method, utilizing the unitarily weighted Euclidean metric. The optimal single-TMD solution found for the DS model excitation presented an average attenuation of $66 \%$ with a mass inclusion of $1.1 \%$, where the operational cases 13 and 14 resulted in the lowest efficiency of attenuation. This behavior was attributed to the great increase of the $z$-direction, and the inherent limitation of TMD systems since their design is essentially dependent on a target frequency and the DOF response magnitude. As for the One- 
minus cosine gust model excitation, the average attenuation of the aeroelastic response was evaluated as $50 \%$, for a mass inclusion of $0.9 \%$. An alike behavior of reducing the attenuation effectiveness when increasing the mean wind speed of the operational cases was also observed for the time-domain response. The optimal individuals for the frequency and time-domain analysis were different, which is expected to occur since the excitation models are different from each other. The optimal sets for the DS and One-minus cosine models were tested among each other, and it was found that the best individual associated with the time-domain excitation presented better attenuation capabilities, hence being defined as the overall optimal individual. Therefore, in order to design a single-TMD device to attenuate the aeroelastic response of the VLBI antenna, one can refer to the optimal parameters found herein as a starting point for further analysis refinement.

\subsection{Recommendations for Future Work}

The scope of the thesis was limited to the numerical investigation of performance of conventional TMD, by means of an in-house developed framework with the goal to attenuate the aeroelastic response of aerospace structures. Several aspects, in terms of the numerical analysis and experimental approaches, can be improved and are suggested as recommendation for future work related to the topic:

1. In the design of the TMD devices, an interesting consideration would be the relative displacement of the seismic masses could be taken as a constraint or an objective function for the optimization problem. This is an important step when designing TMD systems due to the fact that for some applications geometrical constraints are present in the structure, hence being necessary to predict how much displacement the resonators will manifest. 
2. Conduct a stress analysis on the beam members of the antenna while concurrently determining the optimal parameters for the TMD would definitely be a great improvement to the model, such that more realistic results can be obtained for the actual implementation of the optimal solution. The idea behind this approach would be to consider the maximum tensile or shear stresses acting on the truss elements of the structure, being defined as an objective function for the optimization problem, alongside with the mass inclusion and aeroelastic response.

3. The optimal parameters found in the work, for the Case Study II, considered the VLBI antenna structure with a fixed azimuth angle at $0^{\circ}$. In reality, the antenna dynamically rotates the reflectors, such that the mass and stiffness distribution changes for each azimuth angles defined. To make the analysis more complete and realistic, different azimuth angles should be thoroughly studied.

4. Still related to the antenna aeroelastic model itself, it would be of interest to perform an aerodynamic model adjustment with the intent to achieve accurate quantitative results, since the DLM presents limitations in its use when the thickness of structures is not negligible for proper estimation of aerodynamic coefficients.

5. One drawback of the framework implement is related to the computation time taken for each run. Even though with the ROM implemented, each iteration takes a relatively high amount of time to compute, and when utilized in an optimization with GA, where thousands of iterations are necessary, the total time can exponentially build up. Hence, further reducing the current ROM would reduce the time to determine the optimal solution. Another strategy that is commonly used in vibration analysis is to simplify the problems into a few degrees-of-freedom only, where an analytical solution can be found for the 
problem. This approach could assist easily to determine the optimal solution of the TMD parameters for complex aerospace structures based on that simplified model.

6. Still related to computation time reduction and reliability of the code, other possible improvement would be the utilization of open-source programming languages to employ the GA framework, such as Python.

7. A thorough investigation about the sensitivity of the updated ROM coupled with the TMD devices is necessary to further understand the effect of the reduction of the DOF in the aeroelastic signal response, and also on the optimal TMD set found from the framework.

8. An experimental investigation to validate the framework results would be a pivotal area of research, possibly starting with a simplified antenna structure, where the resonators can be placed and tuned accordingly.

9. As there is a great variety of TMD configurations, different approaches could be investigated and compared their performance, such as active control or semi-active using TMDs, or other passive configurations (pendular-TMD for example). Furthermore, within the multiple-TMD approach, it would be of interest to analyze different locations than the target node to place the resonators, such as in the primary reflector or boom arms, and therefore varying the mass of each TMD across the spectrum of devices used on the antenna 


\section{Appendices}

\section{Appendix A - MATLAB ${ }^{\circledR}$ routines for the framework validation}

\section{A.1 User guide}

This simple case study for validation of the procedure of the code contains a total of 6 functions, which are listed herein. To run the code, save all functions in the same folder. Using the function "main.m" will allow the user to call the subfunctions and perform the optimization of the TMD parameters by using the GA procedure from MATLAB ${ }^{\circledR}$. All input parameters can be changed accordingly.

Initially, the input variables are called, and they contain the basic information and constitutive values for the modelling of the SDOF system with an attached TMD. Next, the analytical solution is calculated for the design variables as the pair $\left(f, \xi_{d}\right)$ using the function “optimalTMD_analytical.m”. In sequence, the GA optimization is performed, where the fitness function is evaluated from the maximum estimated peak of the FRF obtained by using the function "fitnessFun.m". In this section, if it is of interest, the user can change the population size, maximum generations to be analyzed, among the other genetic operators in order to achieve more precise results. The final step is the plotting of the results, given by the function "plotGraphs.m", where a set of two graphs are displayed on the screen.

\section{A.2 main.m}

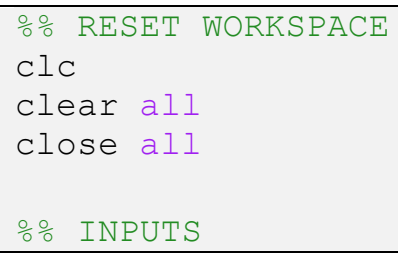




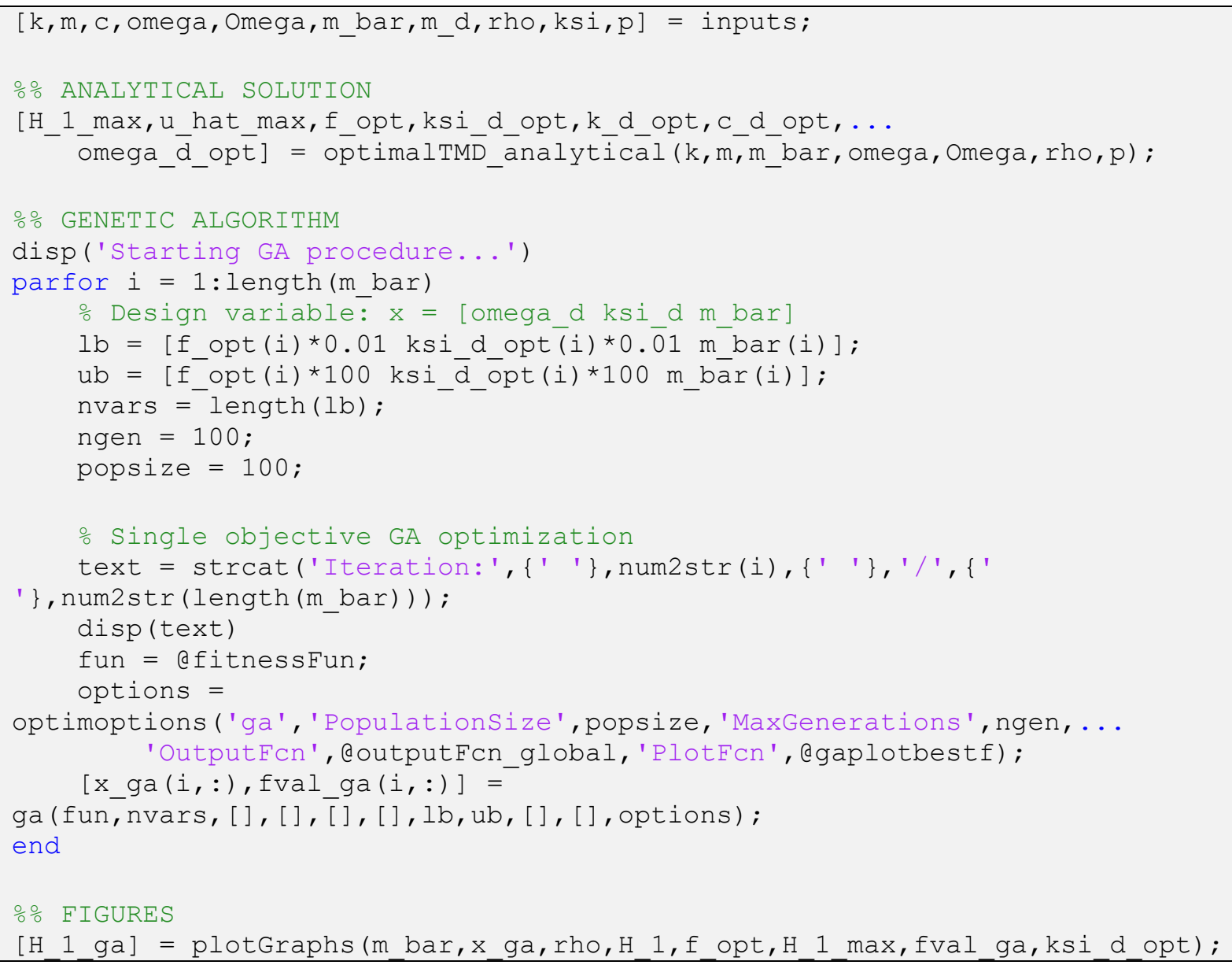

\section{A.3 inputs.m}

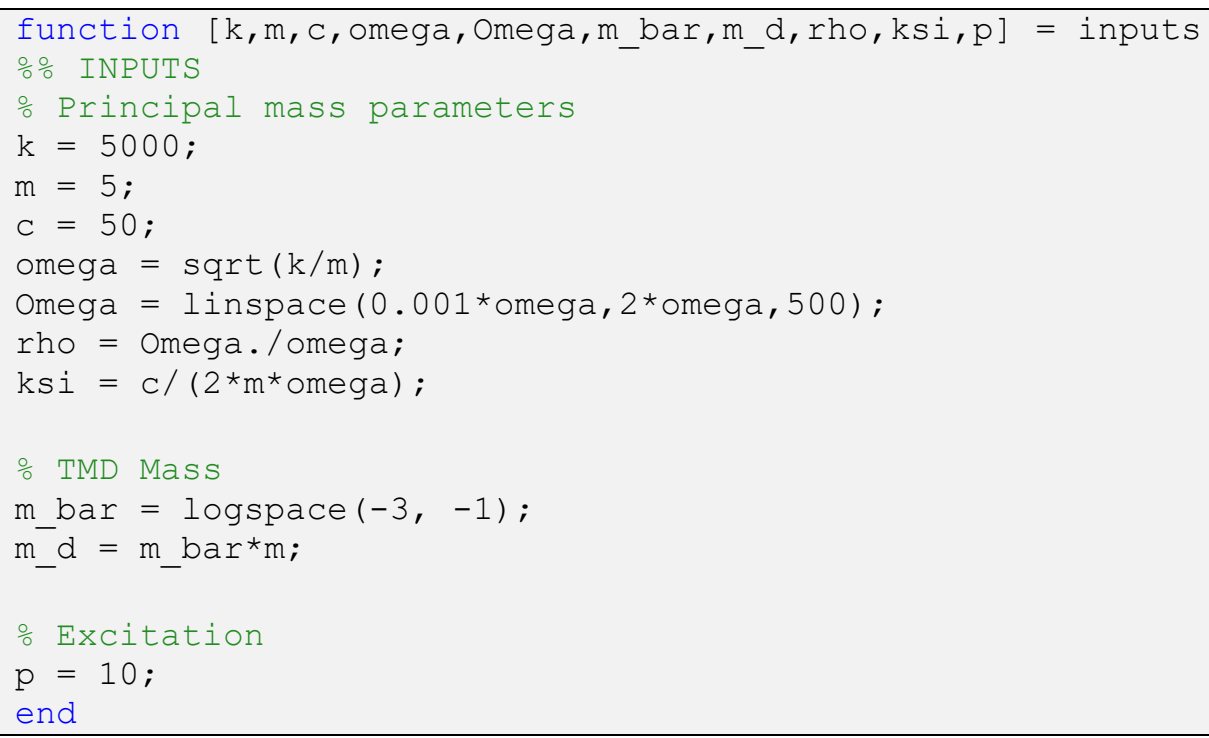




\section{A.4 optimalTMD_analytical.m}

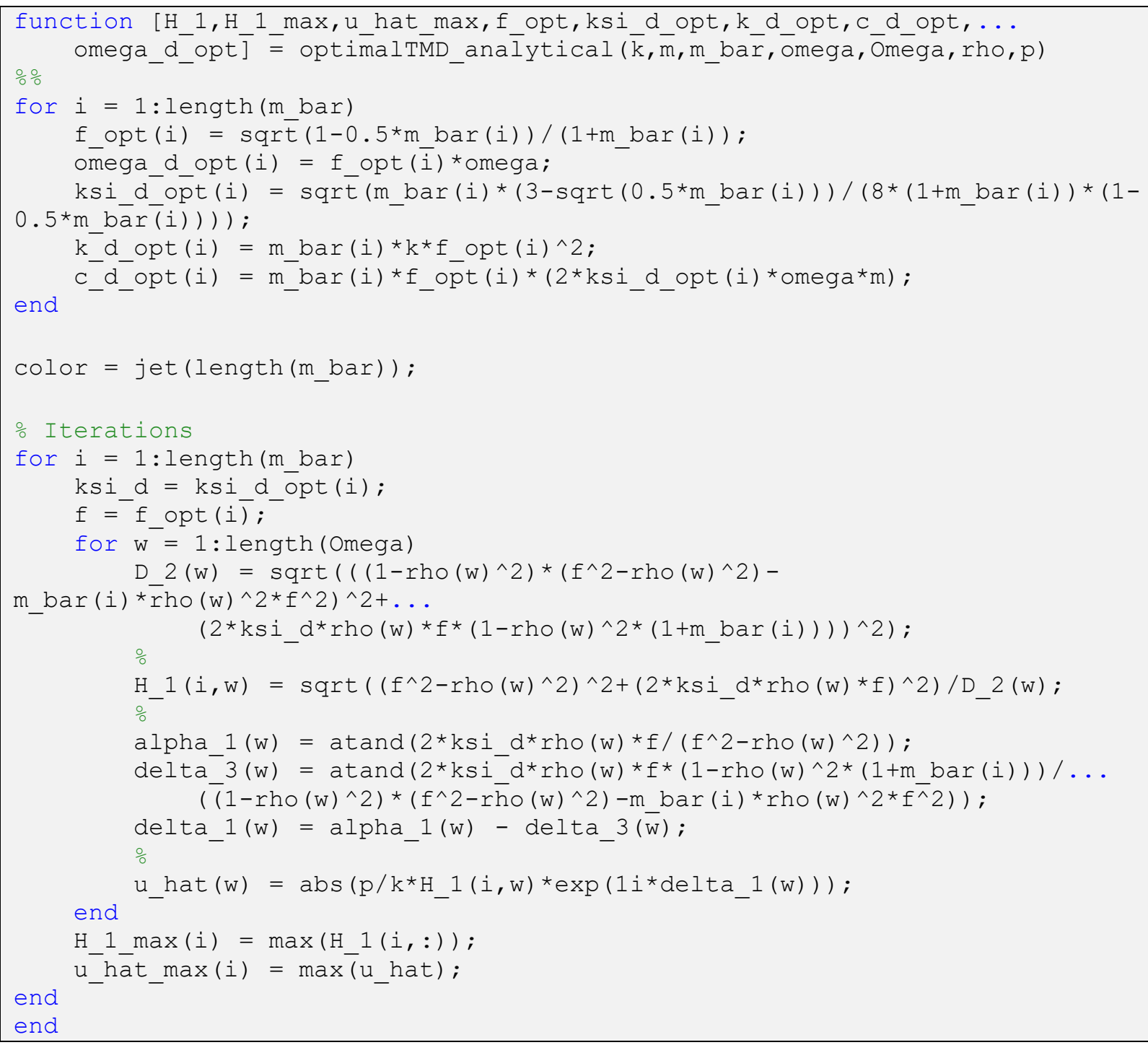

\section{A.5 fitnessFun.m}

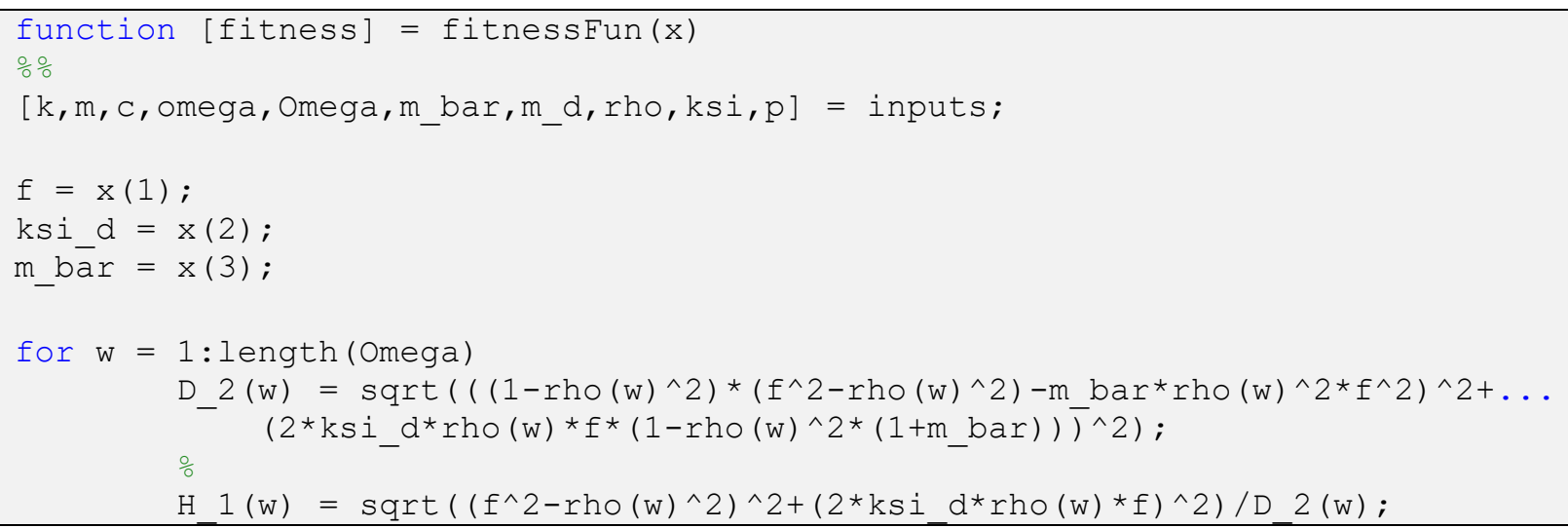




\begin{tabular}{|c|}
\hline 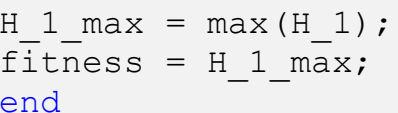 \\
\hline
\end{tabular}

\section{A.6 plotGraphs.m}

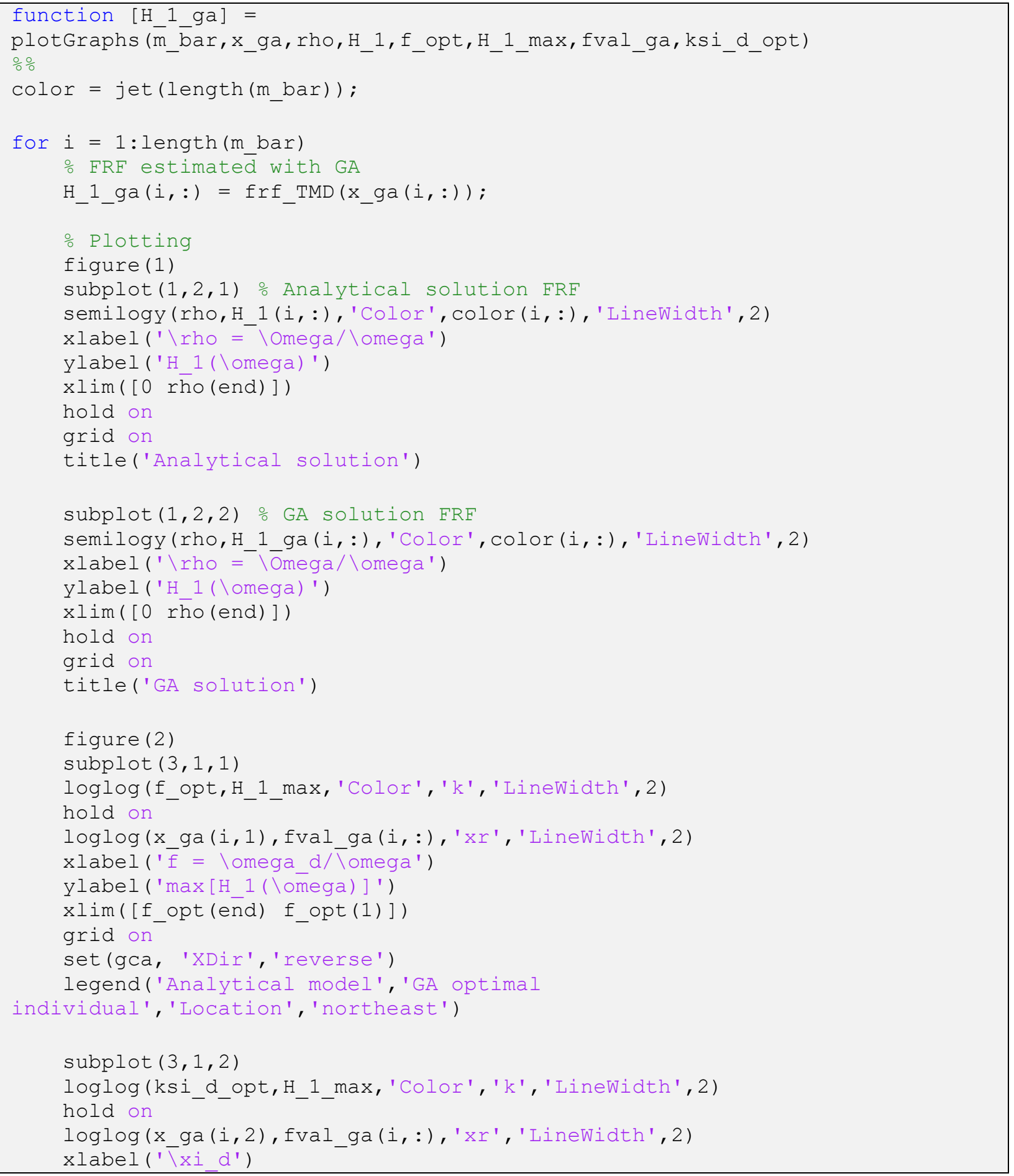




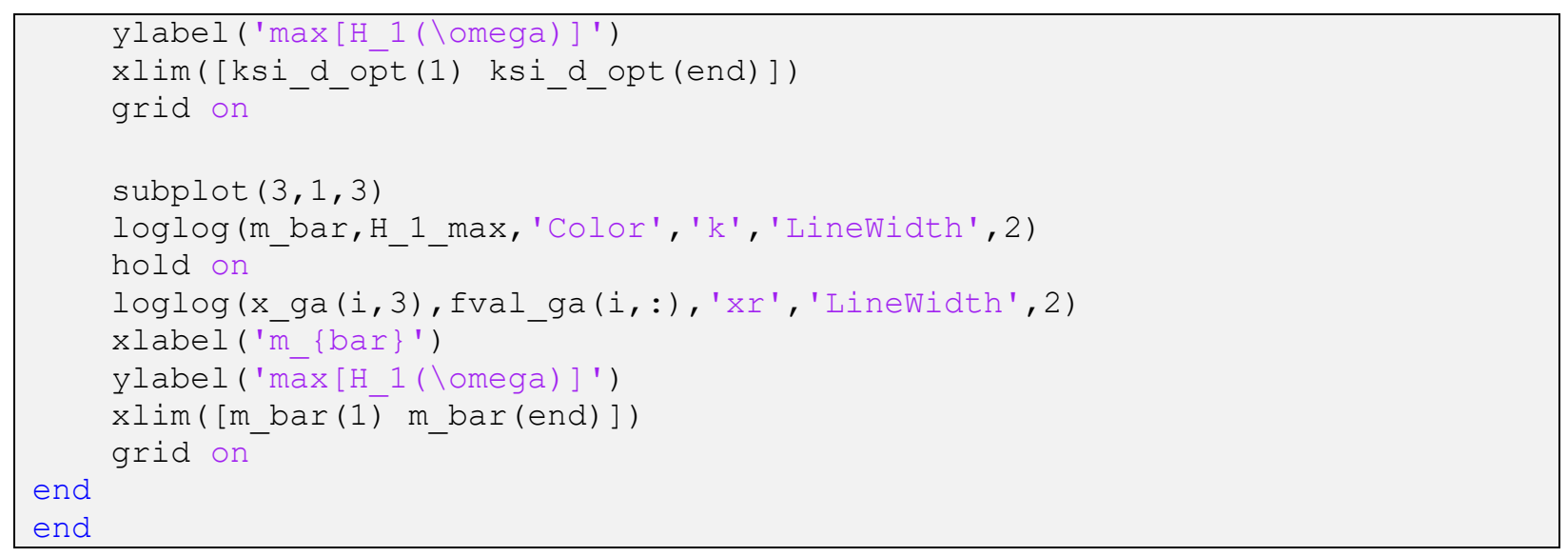

\section{A.7 frf_TMD.m}

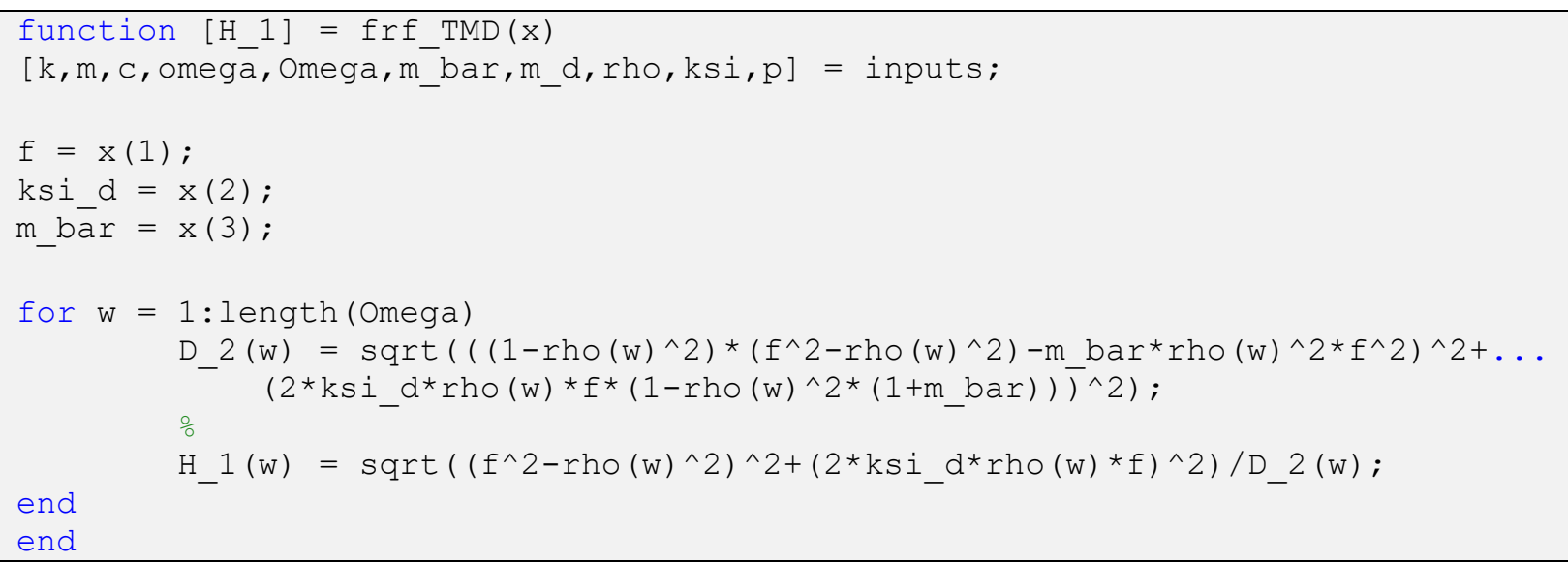




\section{Appendix B - Case study II: Pareto individuals}

\section{B.1 Davenport Spectrum frequency domain analysis}

The Pareto individuals obtained from the MOGA procedure for the single-TMD control device configuration, considering the PSD analysis with the DS gust profile excitation, are presented in Table B.1 to Table B.3. A total of 35 individuals were obtained in the Pareto front, and the inclusion of the single-TMD concurrently attenuates the response of all cases presented. The tables presented in this appendix are useful for the designer to select the best constitutive parameters when implementing a TMD device in the VLBI antenna structure. The parameters presented are the same for all DOF considered, i.e., directions $x, y$ and $z$ assume the same properties of mass, damping coefficient and stiffness.

Table B.1: Pareto front individuals for the single-TMD control devices under the DS gust profile excitation, for the operational cases 1 to 5

\begin{tabular}{|c|c|c|c|c|c|c|c|c|}
\hline \multirow{2}{*}{ Pareto Point ID } & \multirow{2}{*}{ m (lb) } & \multirow{2}{*}{ c (lbf.s/in) } & \multirow{2}{*}{ k (lbf/in) } & \multicolumn{5}{|c|}{$U_{\text {fitness }}\left[\mathrm{in}^{2} / \mathrm{Hz}\right]$} \\
\hline & & & & Case 1 & Case 2 & Case 3 & Case 4 & Case 5 \\
\hline 1 & $3.67 \mathrm{E}+03$ & $4.49 \mathrm{E}+01$ & $1.11 \mathrm{E}+03$ & $1.25 \mathrm{E}-08$ & 3.69E-08 & 7.95E-08 & $1.44 \mathrm{E}-07$ & $2.35 \mathrm{E}-07$ \\
\hline 2 & $3.63 \mathrm{E}+03$ & $4.56 \mathrm{E}+01$ & $8.96 \mathrm{E}+02$ & $1.69 \mathrm{E}-08$ & 4.94E-08 & $1.08 \mathrm{E}-07$ & $1.96 \mathrm{E}-07$ & $3.20 \mathrm{E}-07$ \\
\hline 3 & $3.55 \mathrm{E}+03$ & $3.54 \mathrm{E}+01$ & $8.64 \mathrm{E}+02$ & $1.88 \mathrm{E}-08$ & $5.57 \mathrm{E}-08$ & $1.22 \mathrm{E}-07$ & $2.22 \mathrm{E}-07$ & $3.63 \mathrm{E}-07$ \\
\hline 4 & $3.47 \mathrm{E}+03$ & $5.00 \mathrm{E}+01$ & $9.87 \mathrm{E}+02$ & $1.38 \mathrm{E}-08$ & $4.03 \mathrm{E}-08$ & $8.76 \mathrm{E}-08$ & $1.60 \mathrm{E}-07$ & $2.61 \mathrm{E}-07$ \\
\hline 5 & $3.39 \mathrm{E}+03$ & $4.34 \mathrm{E}+01$ & $1.03 \mathrm{E}+03$ & $1.20 \mathrm{E}-08$ & $3.51 \mathrm{E}-08$ & 7.64E-08 & $1.39 \mathrm{E}-07$ & $2.28 \mathrm{E}-07$ \\
\hline 6 & $3.37 \mathrm{E}+03$ & $4.87 \mathrm{E}+01$ & $1.08 \mathrm{E}+03$ & $1.21 \mathrm{E}-08$ & $3.58 \mathrm{E}-08$ & 7.71E-08 & $1.40 \mathrm{E}-07$ & $2.28 \mathrm{E}-07$ \\
\hline 7 & $3.27 \mathrm{E}+03$ & $5.26 \mathrm{E}+01$ & $1.07 \mathrm{E}+03$ & $1.23 \mathrm{E}-08$ & $3.58 \mathrm{E}-08$ & 7.74E-08 & $1.41 \mathrm{E}-07$ & $2.31 \mathrm{E}-07$ \\
\hline 8 & $3.17 \mathrm{E}+03$ & $5.13 \mathrm{E}+01$ & $9.28 \mathrm{E}+02$ & $1.58 \mathrm{E}-08$ & $4.59 \mathrm{E}-08$ & $9.94 \mathrm{E}-08$ & $1.81 \mathrm{E}-07$ & $2.96 \mathrm{E}-07$ \\
\hline 9 & $2.88 \mathrm{E}+03$ & $4.44 \mathrm{E}+01$ & $9.96 \mathrm{E}+02$ & $1.30 \mathrm{E}-08$ & $3.77 \mathrm{E}-08$ & $8.16 \mathrm{E}-08$ & $1.48 \mathrm{E}-07$ & $2.43 \mathrm{E}-07$ \\
\hline
\end{tabular}




\begin{tabular}{|c|c|c|c|c|c|c|c|c|}
\hline 10 & $2.79 \mathrm{E}+03$ & $3.59 \mathrm{E}+01$ & $9.90 \mathrm{E}+02$ & $1.33 \mathrm{E}-08$ & $3.92 \mathrm{E}-08$ & $8.43 \mathrm{E}-08$ & $1.53 \mathrm{E}-07$ & $2.49 \mathrm{E}-07$ \\
\hline 11 & $2.62 \mathrm{E}+03$ & $3.13 \mathrm{E}+01$ & $5.93 \mathrm{E}+02$ & $3.04 \mathrm{E}-08$ & 8.99E-08 & $1.95 \mathrm{E}-07$ & $3.55 \mathrm{E}-07$ & $5.80 \mathrm{E}-07$ \\
\hline 12 & $2.58 \mathrm{E}+03$ & $3.56 \mathrm{E}+01$ & $6.58 \mathrm{E}+02$ & $2.53 \mathrm{E}-08$ & 7.44E-08 & $1.61 \mathrm{E}-07$ & $2.94 \mathrm{E}-07$ & $4.79 \mathrm{E}-07$ \\
\hline 13 & $2.46 \mathrm{E}+03$ & $3.09 \mathrm{E}+01$ & $7.49 \mathrm{E}+02$ & $1.89 \mathrm{E}-08$ & $5.55 \mathrm{E}-08$ & $1.21 \mathrm{E}-07$ & $2.20 \mathrm{E}-07$ & $3.59 \mathrm{E}-07$ \\
\hline 14 & $2.20 \mathrm{E}+03$ & $4.49 \mathrm{E}+01$ & $4.92 \mathrm{E}+02$ & 3.99E-08 & $1.17 \mathrm{E}-07$ & $2.52 \mathrm{E}-07$ & $4.57 \mathrm{E}-07$ & $7.47 \mathrm{E}-07$ \\
\hline 15 & $2.10 \mathrm{E}+03$ & $2.77 \mathrm{E}+01$ & $6.86 \mathrm{E}+02$ & $2.06 \mathrm{E}-08$ & $6.03 \mathrm{E}-08$ & $1.31 \mathrm{E}-07$ & $2.38 \mathrm{E}-07$ & $3.89 \mathrm{E}-07$ \\
\hline 16 & $2.00 \mathrm{E}+03$ & $3.01 \mathrm{E}+01$ & $6.17 \mathrm{E}+02$ & $2.62 \mathrm{E}-08$ & $7.66 \mathrm{E}-08$ & $1.66 \mathrm{E}-07$ & $3.01 \mathrm{E}-07$ & $4.93 \mathrm{E}-07$ \\
\hline 17 & $1.84 \mathrm{E}+03$ & $3.18 \mathrm{E}+01$ & $7.88 \mathrm{E}+02$ & 2.37E-08 & $6.92 \mathrm{E}-08$ & $1.49 \mathrm{E}-07$ & $2.69 \mathrm{E}-07$ & $4.41 \mathrm{E}-07$ \\
\hline 18 & $1.74 \mathrm{E}+03$ & $3.50 \mathrm{E}+01$ & $6.83 \mathrm{E}+02$ & $3.13 \mathrm{E}-08$ & $9.13 \mathrm{E}-08$ & $1.96 \mathrm{E}-07$ & $3.56 \mathrm{E}-07$ & $5.83 \mathrm{E}-07$ \\
\hline 19 & $1.71 \mathrm{E}+03$ & $3.23 \mathrm{E}+01$ & $5.74 \mathrm{E}+02$ & $3.36 \mathrm{E}-08$ & $9.80 \mathrm{E}-08$ & $2.11 \mathrm{E}-07$ & $3.83 \mathrm{E}-07$ & $6.28 \mathrm{E}-07$ \\
\hline 20 & $1.59 \mathrm{E}+03$ & $3.13 \mathrm{E}+01$ & $5.00 \mathrm{E}+02$ & $4.05 \mathrm{E}-08$ & $1.19 \mathrm{E}-07$ & $2.55 \mathrm{E}-07$ & $4.64 \mathrm{E}-07$ & $7.58 \mathrm{E}-07$ \\
\hline 21 & $1.35 \mathrm{E}+03$ & $3.39 \mathrm{E}+01$ & $4.40 \mathrm{E}+02$ & $5.75 \mathrm{E}-08$ & $1.68 \mathrm{E}-07$ & $3.62 \mathrm{E}-07$ & $6.54 \mathrm{E}-07$ & $1.07 \mathrm{E}-06$ \\
\hline 22 & $1.32 \mathrm{E}+03$ & $3.39 \mathrm{E}+01$ & $4.40 \mathrm{E}+02$ & $5.98 \mathrm{E}-08$ & $1.75 \mathrm{E}-07$ & $3.77 \mathrm{E}-07$ & $6.81 \mathrm{E}-07$ & $1.12 \mathrm{E}-06$ \\
\hline 23 & $1.19 \mathrm{E}+03$ & $3.87 \mathrm{E}+01$ & $4.26 \mathrm{E}+02$ & 7.99E-08 & $2.34 \mathrm{E}-07$ & $5.03 \mathrm{E}-07$ & $9.10 \mathrm{E}-07$ & $1.49 \mathrm{E}-06$ \\
\hline 24 & $9.40 \mathrm{E}+02$ & $2.47 \mathrm{E}+01$ & $4.52 \mathrm{E}+02$ & 7.94E-08 & $2.32 \mathrm{E}-07$ & 4.99E-07 & $9.03 \mathrm{E}-07$ & $1.48 \mathrm{E}-06$ \\
\hline $25^{*}$ & $8.48 \mathrm{E}+02$ & $1.67 \mathrm{E}+01$ & $5.04 \mathrm{E}+02$ & $8.77 \mathrm{E}-08$ & $2.55 \mathrm{E}-07$ & $5.50 \mathrm{E}-07$ & $9.90 \mathrm{E}-07$ & $1.63 \mathrm{E}-06$ \\
\hline 26 & $7.75 \mathrm{E}+02$ & $3.12 \mathrm{E}+01$ & $5.55 \mathrm{E}+02$ & $1.47 \mathrm{E}-07$ & $4.30 \mathrm{E}-07$ & $9.23 \mathrm{E}-07$ & $1.67 \mathrm{E}-06$ & $2.72 \mathrm{E}-06$ \\
\hline 27 & $7.04 \mathrm{E}+02$ & $3.12 \mathrm{E}+01$ & $4.56 \mathrm{E}+02$ & $1.49 \mathrm{E}-07$ & $4.37 \mathrm{E}-07$ & $9.41 \mathrm{E}-07$ & $1.70 \mathrm{E}-06$ & $2.78 \mathrm{E}-06$ \\
\hline 28 & $6.26 \mathrm{E}+02$ & $3.12 \mathrm{E}+01$ & $4.17 \mathrm{E}+02$ & $1.64 \mathrm{E}-07$ & $4.81 \mathrm{E}-07$ & $1.03 \mathrm{E}-06$ & $1.87 \mathrm{E}-06$ & $3.04 \mathrm{E}-06$ \\
\hline 29 & $5.88 \mathrm{E}+02$ & $3.12 \mathrm{E}+01$ & $4.17 \mathrm{E}+02$ & $1.73 \mathrm{E}-07$ & $5.08 \mathrm{E}-07$ & $1.09 \mathrm{E}-06$ & $1.97 \mathrm{E}-06$ & $3.22 \mathrm{E}-06$ \\
\hline 30 & $5.03 \mathrm{E}+02$ & $5.47 \mathrm{E}+01$ & $5.89 \mathrm{E}+02$ & $2.20 \mathrm{E}-07$ & $6.39 \mathrm{E}-07$ & $1.39 \mathrm{E}-06$ & $2.48 \mathrm{E}-06$ & $4.08 \mathrm{E}-06$ \\
\hline 31 & $4.67 \mathrm{E}+02$ & $4.56 \mathrm{E}+01$ & $5.71 \mathrm{E}+02$ & 2.19E-07 & $6.41 \mathrm{E}-07$ & $1.38 \mathrm{E}-06$ & $2.49 \mathrm{E}-06$ & $4.08 \mathrm{E}-06$ \\
\hline 32 & $3.81 \mathrm{E}+02$ & $4.70 \mathrm{E}+01$ & $7.42 \mathrm{E}+02$ & $2.34 \mathrm{E}-07$ & $6.87 \mathrm{E}-07$ & $1.48 \mathrm{E}-06$ & $2.67 \mathrm{E}-06$ & $4.35 \mathrm{E}-06$ \\
\hline 33 & $2.73 \mathrm{E}+02$ & $9.91 \mathrm{E}+01$ & $9.72 \mathrm{E}+02$ & $2.38 \mathrm{E}-07$ & $6.93 \mathrm{E}-07$ & $1.50 \mathrm{E}-06$ & $2.69 \mathrm{E}-06$ & $4.41 \mathrm{E}-06$ \\
\hline 34 & $1.61 \mathrm{E}+02$ & $3.64 \mathrm{E}+01$ & $7.08 \mathrm{E}+02$ & $2.47 \mathrm{E}-07$ & $7.34 \mathrm{E}-07$ & $1.58 \mathrm{E}-06$ & $2.87 \mathrm{E}-06$ & $4.66 \mathrm{E}-06$ \\
\hline 35 & $7.72 \mathrm{E}+01$ & $1.83 \mathrm{E}+02$ & $2.28 \mathrm{E}+02$ & $3.23 \mathrm{E}-07$ & $9.36 \mathrm{E}-07$ & $2.06 \mathrm{E}-06$ & $3.65 \mathrm{E}-06$ & $6.08 \mathrm{E}-06$ \\
\hline
\end{tabular}

Note: * Best individual found with the Utopian Point Method 
Table B.2: Pareto front individuals for the single-TMD control devices under the DS gust profile excitation, for the operational cases 6 to 10

\begin{tabular}{|c|c|c|c|c|c|c|c|c|}
\hline \multirow{2}{*}{ Pareto Point ID } & \multirow{2}{*}{ m (lb) } & \multirow{2}{*}{ c (lbf.s/in) } & \multirow{2}{*}{ k (lbf/in) } & \multicolumn{5}{|c|}{$U_{\text {fitness }}\left[\mathrm{in}^{2} / \mathrm{Hz}\right]$} \\
\hline & & & & Case 6 & Case 7 & Case 8 & Case 9 & Case 10 \\
\hline 1 & $3.67 \mathrm{E}+03$ & $4.49 \mathrm{E}+01$ & $1.11 \mathrm{E}+03$ & 2.79E-07 & $3.55 \mathrm{E}-07$ & $5.53 \mathrm{E}-07$ & $1.28 \mathrm{E}-06$ & 7.67E-06 \\
\hline 2 & $3.63 \mathrm{E}+03$ & $4.56 \mathrm{E}+01$ & $8.96 \mathrm{E}+02$ & $3.82 \mathrm{E}-07$ & $4.81 \mathrm{E}-07$ & $6.77 \mathrm{E}-07$ & $1.25 \mathrm{E}-06$ & 4.92E-06 \\
\hline 3 & $3.55 \mathrm{E}+03$ & $3.54 \mathrm{E}+01$ & $8.64 \mathrm{E}+02$ & $4.33 \mathrm{E}-07$ & $5.47 \mathrm{E}-07$ & 7.64E-07 & $1.41 \mathrm{E}-06$ & 3.74E-06 \\
\hline 4 & $3.47 \mathrm{E}+03$ & $5.00 \mathrm{E}+01$ & $9.87 \mathrm{E}+02$ & $3.11 \mathrm{E}-07$ & $3.91 \mathrm{E}-07$ & 5.52E-07 & $1.02 \mathrm{E}-06$ & 7.83E-06 \\
\hline 5 & $3.39 \mathrm{E}+03$ & $4.34 \mathrm{E}+01$ & $1.03 \mathrm{E}+03$ & $2.72 \mathrm{E}-07$ & $3.42 \mathrm{E}-07$ & $5.36 \mathrm{E}-07$ & $1.37 \mathrm{E}-06$ & $8.80 \mathrm{E}-06$ \\
\hline 6 & $3.37 \mathrm{E}+03$ & $4.87 \mathrm{E}+01$ & $1.08 \mathrm{E}+03$ & $2.71 \mathrm{E}-07$ & $3.44 \mathrm{E}-07$ & $5.26 \mathrm{E}-07$ & $1.62 \mathrm{E}-06$ & $1.09 \mathrm{E}-05$ \\
\hline 7 & $3.27 \mathrm{E}+03$ & $5.26 \mathrm{E}+01$ & $1.07 \mathrm{E}+03$ & $2.74 \mathrm{E}-07$ & $3.45 \mathrm{E}-07$ & 4.90E-07 & $1.82 \mathrm{E}-06$ & $1.23 \mathrm{E}-05$ \\
\hline 8 & $3.17 \mathrm{E}+03$ & $5.13 \mathrm{E}+01$ & $9.28 \mathrm{E}+02$ & $3.52 \mathrm{E}-07$ & 4.44E-07 & $6.28 \mathrm{E}-07$ & $1.53 \mathrm{E}-06$ & $9.76 \mathrm{E}-06$ \\
\hline 9 & $2.88 \mathrm{E}+03$ & $4.44 \mathrm{E}+01$ & $9.96 \mathrm{E}+02$ & $2.89 \mathrm{E}-07$ & $3.64 \mathrm{E}-07$ & $5.16 \mathrm{E}-07$ & $2.22 \mathrm{E}-06$ & $1.49 \mathrm{E}-05$ \\
\hline 10 & $2.79 \mathrm{E}+03$ & $3.59 \mathrm{E}+01$ & $9.90 \mathrm{E}+02$ & $2.96 \mathrm{E}-07$ & $3.77 \mathrm{E}-07$ & $5.46 \mathrm{E}-07$ & $2.40 \mathrm{E}-06$ & $1.67 \mathrm{E}-05$ \\
\hline 11 & $2.62 \mathrm{E}+03$ & $3.13 \mathrm{E}+01$ & $5.93 \mathrm{E}+02$ & $6.91 \mathrm{E}-07$ & $8.72 \mathrm{E}-07$ & $1.23 \mathrm{E}-06$ & $2.26 \mathrm{E}-06$ & $5.58 \mathrm{E}-06$ \\
\hline 12 & $2.58 \mathrm{E}+03$ & $3.56 \mathrm{E}+01$ & $6.58 \mathrm{E}+02$ & $5.71 \mathrm{E}-07$ & $7.20 \mathrm{E}-07$ & $1.02 \mathrm{E}-06$ & $1.87 \mathrm{E}-06$ & 7.63E-06 \\
\hline 13 & $2.46 \mathrm{E}+03$ & $3.09 \mathrm{E}+01$ & $7.49 \mathrm{E}+02$ & $4.29 \mathrm{E}-07$ & $5.40 \mathrm{E}-07$ & $7.60 \mathrm{E}-07$ & $1.72 \mathrm{E}-06$ & $1.14 \mathrm{E}-05$ \\
\hline 14 & $2.20 \mathrm{E}+03$ & $4.49 \mathrm{E}+01$ & $4.92 \mathrm{E}+02$ & $8.88 \mathrm{E}-07$ & $1.12 \mathrm{E}-06$ & $1.59 \mathrm{E}-06$ & 2.92E-06 & $9.06 \mathrm{E}-06$ \\
\hline 15 & $2.10 \mathrm{E}+03$ & $2.77 \mathrm{E}+01$ & $6.86 \mathrm{E}+02$ & $4.63 \mathrm{E}-07$ & $5.84 \mathrm{E}-07$ & $8.24 \mathrm{E}-07$ & $2.30 \mathrm{E}-06$ & $1.39 \mathrm{E}-05$ \\
\hline 16 & $2.00 \mathrm{E}+03$ & $3.01 \mathrm{E}+01$ & $6.17 \mathrm{E}+02$ & $5.86 \mathrm{E}-07$ & $7.40 \mathrm{E}-07$ & $1.05 \mathrm{E}-06$ & $2.21 \mathrm{E}-06$ & $1.21 \mathrm{E}-05$ \\
\hline 17 & $1.84 \mathrm{E}+03$ & $3.18 \mathrm{E}+01$ & $7.88 \mathrm{E}+02$ & $5.22 \mathrm{E}-07$ & $6.60 \mathrm{E}-07$ & 9.46E-07 & 4.44E-06 & $1.66 \mathrm{E}-05$ \\
\hline 18 & $1.74 \mathrm{E}+03$ & $3.50 \mathrm{E}+01$ & $6.83 \mathrm{E}+02$ & $6.91 \mathrm{E}-07$ & $8.74 \mathrm{E}-07$ & $1.24 \mathrm{E}-06$ & $3.88 \mathrm{E}-06$ & 1.44E-05 \\
\hline 19 & $1.71 \mathrm{E}+03$ & $3.23 \mathrm{E}+01$ & $5.74 \mathrm{E}+02$ & $7.46 \mathrm{E}-07$ & $9.41 \mathrm{E}-07$ & $1.34 \mathrm{E}-06$ & $3.05 \mathrm{E}-06$ & $1.27 \mathrm{E}-05$ \\
\hline 20 & $1.59 \mathrm{E}+03$ & $3.13 \mathrm{E}+01$ & $5.00 \mathrm{E}+02$ & $9.01 \mathrm{E}-07$ & $1.14 \mathrm{E}-06$ & $1.61 \mathrm{E}-06$ & $2.96 \mathrm{E}-06$ & $1.16 \mathrm{E}-05$ \\
\hline 21 & $1.35 \mathrm{E}+03$ & $3.39 \mathrm{E}+01$ & $4.40 \mathrm{E}+02$ & $1.27 \mathrm{E}-06$ & $1.61 \mathrm{E}-06$ & $2.28 \mathrm{E}-06$ & $4.21 \mathrm{E}-06$ & $1.11 \mathrm{E}-05$ \\
\hline 22 & $1.32 \mathrm{E}+03$ & $3.39 \mathrm{E}+01$ & $4.40 \mathrm{E}+02$ & $1.32 \mathrm{E}-06$ & $1.67 \mathrm{E}-06$ & $2.38 \mathrm{E}-06$ & 4.37E-06 & $1.11 \mathrm{E}-05$ \\
\hline 23 & $1.19 \mathrm{E}+03$ & $3.87 \mathrm{E}+01$ & $4.26 \mathrm{E}+02$ & $1.77 \mathrm{E}-06$ & 2.23E-06 & $3.18 \mathrm{E}-06$ & $5.84 \mathrm{E}-06$ & $1.08 \mathrm{E}-05$ \\
\hline
\end{tabular}




$\begin{array}{ccccccccc}24 & 9.40 \mathrm{E}+02 & 2.47 \mathrm{E}+01 & 4.52 \mathrm{E}+02 & 1.75 \mathrm{E}-06 & 2.22 \mathrm{E}-06 & 3.15 \mathrm{E}-06 & 5.80 \mathrm{E}-06 & 1.08 \mathrm{E}-05 \\ 25 * & 8.48 \mathrm{E}+02 & 1.67 \mathrm{E}+01 & 5.04 \mathrm{E}+02 & 1.92 \mathrm{E}-06 & 2.44 \mathrm{E}-06 & 3.46 \mathrm{E}-06 & 6.40 \mathrm{E}-06 & 1.22 \mathrm{E}-05 \\ 26 & 7.75 \mathrm{E}+02 & 3.12 \mathrm{E}+01 & 5.55 \mathrm{E}+02 & 3.23 \mathrm{E}-06 & 4.10 \mathrm{E}-06 & 5.83 \mathrm{E}-06 & 1.07 \mathrm{E}-05 & 1.82 \mathrm{E}-05 \\ 27 & 7.04 \mathrm{E}+02 & 3.12 \mathrm{E}+01 & 4.56 \mathrm{E}+02 & 3.29 \mathrm{E}-06 & 4.18 \mathrm{E}-06 & 5.91 \mathrm{E}-06 & 1.09 \mathrm{E}-05 & 1.83 \mathrm{E}-05 \\ 28 & 6.26 \mathrm{E}+02 & 3.12 \mathrm{E}+01 & 4.17 \mathrm{E}+02 & 3.62 \mathrm{E}-06 & 4.58 \mathrm{E}-06 & 6.50 \mathrm{E}-06 & 1.19 \mathrm{E}-05 & 2.00 \mathrm{E}-05 \\ 29 & 5.88 \mathrm{E}+02 & 3.12 \mathrm{E}+01 & 4.17 \mathrm{E}+02 & 3.82 \mathrm{E}-06 & 4.84 \mathrm{E}-06 & 6.87 \mathrm{E}-06 & 1.26 \mathrm{E}-05 & 2.11 \mathrm{E}-05 \\ 30 & 5.03 \mathrm{E}+02 & 5.47 \mathrm{E}+01 & 5.89 \mathrm{E}+02 & 4.80 \mathrm{E}-06 & 6.15 \mathrm{E}-06 & 8.62 \mathrm{E}-06 & 1.60 \mathrm{E}-05 & 2.64 \mathrm{E}-05 \\ 31 & 4.67 \mathrm{E}+02 & 4.56 \mathrm{E}+01 & 5.71 \mathrm{E}+02 & 4.81 \mathrm{E}-06 & 6.13 \mathrm{E}-06 & 8.64 \mathrm{E}-06 & 1.59 \mathrm{E}-05 & 2.64 \mathrm{E}-05 \\ 32 & 3.81 \mathrm{E}+02 & 4.70 \mathrm{E}+01 & 7.42 \mathrm{E}+02 & 5.17 \mathrm{E}-06 & 6.54 \mathrm{E}-06 & 9.27 \mathrm{E}-06 & 1.70 \mathrm{E}-05 & 2.81 \mathrm{E}-05 \\ 33 & 2.73 \mathrm{E}+02 & 9.91 \mathrm{E}+01 & 9.72 \mathrm{E}+02 & 5.21 \mathrm{E}-06 & 6.65 \mathrm{E}-06 & 9.34 \mathrm{E}-06 & 1.72 \mathrm{E}-05 & 2.79 \mathrm{E}-05 \\ 35 & 1.61 \mathrm{E}+02 & 3.64 \mathrm{E}+01 & 7.08 \mathrm{E}+02 & 5.54 \mathrm{E}-06 & 7.02 \mathrm{E}-06 & 1.00 \mathrm{E}-05 & 1.81 \mathrm{E}-05 & 2.94 \mathrm{E}-05\end{array}$

Note: * Best individual found with the Utopian Point Method

Table B.3: Pareto front individuals for the single-TMD control devices under the DS gust profile excitation, for the operational cases 11 to 14

\begin{tabular}{|c|c|c|c|c|c|c|c|}
\hline \multirow{2}{*}{ Pareto Point ID } & \multirow{2}{*}{ m (lb) } & \multirow{2}{*}{ c (lbf.s/in) } & \multirow{2}{*}{ k (lbf/in) } & \multicolumn{4}{|c|}{$U_{\text {fitness }}\left[\mathrm{in}^{2} / \mathrm{Hz}\right]$} \\
\hline & & & & Case 11 & Case 12 & Case 13 & Case 14 \\
\hline 1 & $3.67 \mathrm{E}+03$ & $4.49 \mathrm{E}+01$ & $1.11 \mathrm{E}+03$ & $1.55 \mathrm{E}-06$ & $2.30 \mathrm{E}-06$ & 4.12E-05 & $3.88 \mathrm{E}-04$ \\
\hline 2 & $3.63 \mathrm{E}+03$ & $4.56 \mathrm{E}+01$ & $8.96 \mathrm{E}+02$ & 4.79E-06 & $3.68 \mathrm{E}-06$ & $6.95 \mathrm{E}-05$ & $6.65 \mathrm{E}-04$ \\
\hline 3 & $3.55 \mathrm{E}+03$ & $3.54 \mathrm{E}+01$ & $8.64 \mathrm{E}+02$ & $2.55 \mathrm{E}-06$ & $4.00 \mathrm{E}-06$ & $6.41 \mathrm{E}-05$ & $6.53 \mathrm{E}-04$ \\
\hline 4 & $3.47 \mathrm{E}+03$ & $5.00 \mathrm{E}+01$ & $9.87 \mathrm{E}+02$ & 4.32E-06 & $3.07 \mathrm{E}-06$ & $6.03 \mathrm{E}-05$ & $5.71 \mathrm{E}-04$ \\
\hline 5 & $3.39 \mathrm{E}+03$ & $4.34 \mathrm{E}+01$ & $1.03 \mathrm{E}+03$ & $1.66 \mathrm{E}-06$ & $2.62 \mathrm{E}-06$ & 4.64E-05 & $4.66 \mathrm{E}-04$ \\
\hline 6 & $3.37 \mathrm{E}+03$ & $4.87 \mathrm{E}+01$ & $1.08 \mathrm{E}+03$ & 4.28E-05 & $2.53 \mathrm{E}-06$ & $5.07 \mathrm{E}-05$ & $4.46 \mathrm{E}-04$ \\
\hline 7 & $3.27 \mathrm{E}+03$ & $5.26 \mathrm{E}+01$ & $1.07 \mathrm{E}+03$ & $3.98 \mathrm{E}-06$ & $2.84 \mathrm{E}-06$ & $6.18 \mathrm{E}-05$ & 5.12E-04 \\
\hline 8 & $3.17 \mathrm{E}+03$ & $5.13 \mathrm{E}+01$ & $9.28 \mathrm{E}+02$ & 4.91E-06 & $3.56 \mathrm{E}-06$ & 7.71E-05 & $6.90 \mathrm{E}-04$ \\
\hline 9 & $2.88 \mathrm{E}+03$ & $4.44 \mathrm{E}+01$ & $9.96 \mathrm{E}+02$ & 4.20E-06 & $2.95 \mathrm{E}-06$ & $6.48 \mathrm{E}-05$ & $5.38 \mathrm{E}-04$ \\
\hline
\end{tabular}




\begin{tabular}{|c|c|c|c|c|c|c|c|}
\hline 10 & $2.79 \mathrm{E}+03$ & $3.59 \mathrm{E}+01$ & $9.90 \mathrm{E}+02$ & $1.99 \mathrm{E}-06$ & $2.85 \mathrm{E}-06$ & 4.97E-05 & $4.40 \mathrm{E}-04$ \\
\hline 11 & $2.62 \mathrm{E}+03$ & $3.13 \mathrm{E}+01$ & $5.93 \mathrm{E}+02$ & $7.43 \mathrm{E}-06$ & $6.68 \mathrm{E}-06$ & $1.27 \mathrm{E}-04$ & $1.18 \mathrm{E}-03$ \\
\hline 12 & $2.58 \mathrm{E}+03$ & $3.56 \mathrm{E}+01$ & $6.58 \mathrm{E}+02$ & $6.74 \mathrm{E}-06$ & $5.62 \mathrm{E}-06$ & $1.13 \mathrm{E}-04$ & $1.04 \mathrm{E}-03$ \\
\hline 13 & $2.46 \mathrm{E}+03$ & $3.09 \mathrm{E}+01$ & $7.49 \mathrm{E}+02$ & $2.85 \mathrm{E}-06$ & $4.16 \mathrm{E}-06$ & $7.81 \mathrm{E}-05$ & 7.52E-04 \\
\hline 14 & $2.20 \mathrm{E}+03$ & $4.49 \mathrm{E}+01$ & $4.92 \mathrm{E}+02$ & $9.92 \mathrm{E}-06$ & $9.16 \mathrm{E}-06$ & $2.12 \mathrm{E}-04$ & $1.77 \mathrm{E}-03$ \\
\hline 15 & $2.10 \mathrm{E}+03$ & $2.77 \mathrm{E}+01$ & $6.86 \mathrm{E}+02$ & $3.24 \mathrm{E}-06$ & $4.65 \mathrm{E}-06$ & $9.56 \mathrm{E}-05$ & $8.71 \mathrm{E}-04$ \\
\hline 16 & $2.00 \mathrm{E}+03$ & $3.01 \mathrm{E}+01$ & $6.17 \mathrm{E}+02$ & 7.05E-06 & 5.89E-06 & $1.32 \mathrm{E}-04$ & $1.15 \mathrm{E}-03$ \\
\hline 17 & $1.84 \mathrm{E}+03$ & $3.18 \mathrm{E}+01$ & $7.88 \mathrm{E}+02$ & $6.53 \mathrm{E}-06$ & $6.28 \mathrm{E}-06$ & $1.44 \mathrm{E}-04$ & $1.01 \mathrm{E}-03$ \\
\hline 18 & $1.74 \mathrm{E}+03$ & $3.50 \mathrm{E}+01$ & $6.83 \mathrm{E}+02$ & $8.48 \mathrm{E}-06$ & $7.50 \mathrm{E}-06$ & $1.90 \mathrm{E}-04$ & $1.47 \mathrm{E}-03$ \\
\hline 19 & $1.71 \mathrm{E}+03$ & $3.23 \mathrm{E}+01$ & $5.74 \mathrm{E}+02$ & 8.83E-06 & $7.76 \mathrm{E}-06$ & $1.84 \mathrm{E}-04$ & $1.52 \mathrm{E}-03$ \\
\hline 20 & $1.59 \mathrm{E}+03$ & $3.13 \mathrm{E}+01$ & $5.00 \mathrm{E}+02$ & $1.00 \mathrm{E}-05$ & 9.30E-06 & $2.15 \mathrm{E}-04$ & $1.79 \mathrm{E}-03$ \\
\hline 21 & $1.35 \mathrm{E}+03$ & $3.39 \mathrm{E}+01$ & $4.40 \mathrm{E}+02$ & $1.32 \mathrm{E}-05$ & $1.34 \mathrm{E}-05$ & $3.15 \mathrm{E}-04$ & $2.54 \mathrm{E}-03$ \\
\hline 22 & $1.32 \mathrm{E}+03$ & $3.39 \mathrm{E}+01$ & $4.40 \mathrm{E}+02$ & $1.36 \mathrm{E}-05$ & $1.40 \mathrm{E}-05$ & $3.25 \mathrm{E}-04$ & $2.78 \mathrm{E}-03$ \\
\hline 23 & $1.19 \mathrm{E}+03$ & $3.87 \mathrm{E}+01$ & $4.26 \mathrm{E}+02$ & $1.70 \mathrm{E}-05$ & $1.88 \mathrm{E}-05$ & 4.47E-04 & $3.50 \mathrm{E}-03$ \\
\hline 24 & $9.40 \mathrm{E}+02$ & $2.47 \mathrm{E}+01$ & $4.52 \mathrm{E}+02$ & $1.69 \mathrm{E}-05$ & $1.86 \mathrm{E}-05$ & $4.45 \mathrm{E}-04$ & $3.44 \mathrm{E}-03$ \\
\hline $25^{*}$ & $8.48 \mathrm{E}+02$ & $1.67 \mathrm{E}+01$ & $5.04 \mathrm{E}+02$ & $1.85 \mathrm{E}-05$ & $2.11 \mathrm{E}-05$ & $5.18 \mathrm{E}-04$ & $3.75 \mathrm{E}-03$ \\
\hline 26 & $7.75 \mathrm{E}+02$ & $3.12 \mathrm{E}+01$ & $5.55 \mathrm{E}+02$ & $2.73 \mathrm{E}-05$ & $3.49 \mathrm{E}-05$ & $6.90 \mathrm{E}-04$ & $5.90 \mathrm{E}-03$ \\
\hline 27 & $7.04 \mathrm{E}+02$ & $3.12 \mathrm{E}+01$ & $4.56 \mathrm{E}+02$ & $2.70 \mathrm{E}-05$ & $3.49 \mathrm{E}-05$ & $6.36 \mathrm{E}-04$ & $5.97 \mathrm{E}-03$ \\
\hline 28 & $6.26 \mathrm{E}+02$ & $3.12 \mathrm{E}+01$ & $4.17 \mathrm{E}+02$ & $2.88 \mathrm{E}-05$ & $3.84 \mathrm{E}-05$ & $6.70 \mathrm{E}-04$ & $6.32 \mathrm{E}-03$ \\
\hline 29 & $5.88 \mathrm{E}+02$ & $3.12 \mathrm{E}+01$ & $4.17 \mathrm{E}+02$ & $3.00 \mathrm{E}-05$ & $4.06 \mathrm{E}-05$ & $6.91 \mathrm{E}-04$ & $6.54 \mathrm{E}-03$ \\
\hline 30 & $5.03 \mathrm{E}+02$ & $5.47 \mathrm{E}+01$ & $5.89 \mathrm{E}+02$ & $3.49 \mathrm{E}-05$ & $5.02 \mathrm{E}-05$ & $7.81 \mathrm{E}-04$ & $7.23 \mathrm{E}-03$ \\
\hline 31 & $4.67 \mathrm{E}+02$ & $4.56 \mathrm{E}+01$ & $5.71 \mathrm{E}+02$ & $3.48 \mathrm{E}-05$ & $5.00 \mathrm{E}-05$ & 7.30E-04 & $7.19 \mathrm{E}-03$ \\
\hline 32 & $3.81 \mathrm{E}+02$ & $4.70 \mathrm{E}+01$ & $7.42 \mathrm{E}+02$ & $3.63 \mathrm{E}-05$ & $5.35 \mathrm{E}-05$ & $7.40 \mathrm{E}-04$ & 7.27E-03 \\
\hline 33 & $2.73 \mathrm{E}+02$ & $9.91 \mathrm{E}+01$ & $9.72 \mathrm{E}+02$ & $4.01 \mathrm{E}-05$ & $5.90 \mathrm{E}-05$ & $7.15 \mathrm{E}-04$ & $6.99 \mathrm{E}-03$ \\
\hline 34 & $1.61 \mathrm{E}+02$ & $3.64 \mathrm{E}+01$ & $7.08 \mathrm{E}+02$ & $4.36 \mathrm{E}-05$ & 6.39E-05 & $6.47 \mathrm{E}-04$ & $6.50 \mathrm{E}-03$ \\
\hline 35 & $7.72 \mathrm{E}+01$ & $1.83 \mathrm{E}+02$ & $2.28 \mathrm{E}+02$ & $5.57 \mathrm{E}-05$ & $8.13 \mathrm{E}-05$ & $6.20 \mathrm{E}-04$ & $6.19 \mathrm{E}-03$ \\
\hline
\end{tabular}

Note: * Best individual found with the Utopian Point Method 


\section{B.2 One-minus Cosine time domain analysis}

The Pareto individuals obtained from the MOGA procedure for the single-TMD control device configuration, considering the time domain analysis with the One-minus cosine gust profile excitation, are presented in Table B.4 to Table B.6. A total of 35 individuals were obtained in the Pareto front, and the inclusion of the single-TMD concurrently attenuates the response of all cases presented. The tables presented in this appendix are useful for the designer to select the best constitutive parameters when implementing a TMD device in the VLBI antenna structure. The parameters presented are the same for all DOF considered, i.e., directions $x, y$ and $z$ assume the same properties of mass, damping coefficient and stiffness.

Table B.4: Pareto front individuals for the single-TMD control devices under the One-minus cosine gust profile excitation, for the operational cases 1 to 5

\begin{tabular}{|c|c|c|c|c|c|c|c|c|}
\hline \multirow{2}{*}{ Pareto Point ID } & \multirow{2}{*}{ m (lb) } & \multirow{2}{*}{ c (lbf.s/in) } & \multirow{2}{*}{ k (lbf/in) } & \multicolumn{5}{|c|}{$U_{\text {fitness }}[\mathrm{in}]$} \\
\hline & & & & Case 1 & Case 2 & Case 3 & Case 4 & Case 5 \\
\hline 1 & $2.43 \mathrm{E}+03$ & $2.74 \mathrm{E}+00$ & $9.86 \mathrm{E}+02$ & $3.67 \mathrm{E}-06$ & $3.62 \mathrm{E}-06$ & $3.59 \mathrm{E}-06$ & 4.04E-06 & $5.91 \mathrm{E}-06$ \\
\hline 2 & $2.40 \mathrm{E}+03$ & $6.78 \mathrm{E}+00$ & $1.03 \mathrm{E}+03$ & $2.52 \mathrm{E}-06$ & $2.49 \mathrm{E}-06$ & $2.55 \mathrm{E}-06$ & $3.11 \mathrm{E}-06$ & 4.81E-06 \\
\hline 3 & $2.32 \mathrm{E}+03$ & $5.51 \mathrm{E}+00$ & $9.93 \mathrm{E}+02$ & $2.70 \mathrm{E}-06$ & $2.67 \mathrm{E}-06$ & $2.71 \mathrm{E}-06$ & $3.24 \mathrm{E}-06$ & 4.95E-06 \\
\hline 4 & $2.30 \mathrm{E}+03$ & $2.52 \mathrm{E}+01$ & $6.97 \mathrm{E}+02$ & $1.67 \mathrm{E}-07$ & $2.05 \mathrm{E}-07$ & $6.54 \mathrm{E}-07$ & $1.54 \mathrm{E}-06$ & 5.61E-06 \\
\hline 5 & $2.26 \mathrm{E}+03$ & $5.29 \mathrm{E}+00$ & $9.60 \mathrm{E}+02$ & $2.80 \mathrm{E}-06$ & $2.76 \mathrm{E}-06$ & $2.79 \mathrm{E}-06$ & $3.33 \mathrm{E}-06$ & $5.07 \mathrm{E}-06$ \\
\hline 6 & $2.15 \mathrm{E}+03$ & $5.29 \mathrm{E}+00$ & $9.57 \mathrm{E}+02$ & $2.51 \mathrm{E}-06$ & $2.48 \mathrm{E}-06$ & $2.57 \mathrm{E}-06$ & $3.21 \mathrm{E}-06$ & $4.84 \mathrm{E}-06$ \\
\hline 7 & $2.09 \mathrm{E}+03$ & $5.22 \mathrm{E}+00$ & $8.91 \mathrm{E}+02$ & $2.88 \mathrm{E}-06$ & $2.83 \mathrm{E}-06$ & $2.85 \mathrm{E}-06$ & $3.44 \mathrm{E}-06$ & $5.23 \mathrm{E}-06$ \\
\hline 8 & $1.96 \mathrm{E}+03$ & $7.35 \mathrm{E}+00$ & $9.48 \mathrm{E}+02$ & $2.68 \mathrm{E}-06$ & $2.66 \mathrm{E}-06$ & 2.89E-06 & $3.91 \mathrm{E}-06$ & $5.69 \mathrm{E}-06$ \\
\hline 9 & $1.94 \mathrm{E}+03$ & $6.22 \mathrm{E}+00$ & $8.54 \mathrm{E}+02$ & $2.66 \mathrm{E}-06$ & $2.62 \mathrm{E}-06$ & 2.69E-06 & $3.43 \mathrm{E}-06$ & $5.23 \mathrm{E}-06$ \\
\hline 10 & $1.83 \mathrm{E}+03$ & $6.33 \mathrm{E}+00$ & $8.41 \mathrm{E}+02$ & $2.62 \mathrm{E}-06$ & $2.59 \mathrm{E}-06$ & $2.72 \mathrm{E}-06$ & $3.63 \mathrm{E}-06$ & $5.41 \mathrm{E}-06$ \\
\hline 11 & $1.82 \mathrm{E}+03$ & $5.52 \mathrm{E}+00$ & $7.88 \mathrm{E}+02$ & $2.94 \mathrm{E}-06$ & $2.88 \mathrm{E}-06$ & $2.90 \mathrm{E}-06$ & $3.64 \mathrm{E}-06$ & $5.57 \mathrm{E}-06$ \\
\hline
\end{tabular}




\begin{tabular}{|c|c|c|c|c|c|c|c|c|}
\hline 12 & $1.74 \mathrm{E}+03$ & $4.48 \mathrm{E}+00$ & $7.49 \mathrm{E}+02$ & $3.13 \mathrm{E}-06$ & $3.06 \mathrm{E}-06$ & $3.04 \mathrm{E}-06$ & $3.79 \mathrm{E}-06$ & $5.70 \mathrm{E}-06$ \\
\hline 13 & $1.64 \mathrm{E}+03$ & $7.81 \mathrm{E}+00$ & $7.82 \mathrm{E}+02$ & $3.26 \mathrm{E}-06$ & $3.23 \mathrm{E}-06$ & $3.42 \mathrm{E}-06$ & $4.57 \mathrm{E}-06$ & $6.85 \mathrm{E}-06$ \\
\hline 14 & $1.58 \mathrm{E}+03$ & $7.35 \mathrm{E}+00$ & $7.33 \mathrm{E}+02$ & $3.14 \mathrm{E}-06$ & $3.10 \mathrm{E}-06$ & $3.24 \mathrm{E}-06$ & $4.36 \mathrm{E}-06$ & $6.63 \mathrm{E}-06$ \\
\hline 15 & $1.53 \mathrm{E}+03$ & $7.34 \mathrm{E}+00$ & $6.89 \mathrm{E}+02$ & $3.18 \mathrm{E}-06$ & $3.12 \mathrm{E}-06$ & $3.24 \mathrm{E}-06$ & $4.34 \mathrm{E}-06$ & $6.73 \mathrm{E}-06$ \\
\hline 16 & $1.40 \mathrm{E}+03$ & $8.48 \mathrm{E}+00$ & $5.88 \mathrm{E}+02$ & $3.85 \mathrm{E}-06$ & $3.76 \mathrm{E}-06$ & $3.82 \mathrm{E}-06$ & $5.02 \mathrm{E}-06$ & $8.27 \mathrm{E}-06$ \\
\hline 17 & $1.33 \mathrm{E}+03$ & $8.51 \mathrm{E}+00$ & $6.39 \mathrm{E}+02$ & 4.44E-06 & $4.40 \mathrm{E}-06$ & $4.60 \mathrm{E}-06$ & $5.92 \mathrm{E}-06$ & $9.41 \mathrm{E}-06$ \\
\hline 18 & $1.25 \mathrm{E}+03$ & $4.77 \mathrm{E}+00$ & $5.42 \mathrm{E}+02$ & $3.68 \mathrm{E}-06$ & $3.57 \mathrm{E}-06$ & $3.60 \mathrm{E}-06$ & $4.78 \mathrm{E}-06$ & 7.54E-06 \\
\hline 19 & $1.18 \mathrm{E}+03$ & $8.87 \mathrm{E}+00$ & $5.78 \mathrm{E}+02$ & $5.28 \mathrm{E}-06$ & $5.23 \mathrm{E}-06$ & $5.43 \mathrm{E}-06$ & $6.86 \mathrm{E}-06$ & $1.12 \mathrm{E}-05$ \\
\hline 20 & $1.09 \mathrm{E}+03$ & $5.27 \mathrm{E}+00$ & $4.72 \mathrm{E}+02$ & 4.19E-06 & $4.06 \mathrm{E}-06$ & $4.10 \mathrm{E}-06$ & $5.48 \mathrm{E}-06$ & 8.91E-06 \\
\hline 21 & $1.05 \mathrm{E}+03$ & $4.86 \mathrm{E}+00$ & $4.18 \mathrm{E}+02$ & 4.99E-06 & $4.86 \mathrm{E}-06$ & $4.81 \mathrm{E}-06$ & $6.18 \mathrm{E}-06$ & $1.05 \mathrm{E}-05$ \\
\hline 22 & $9.74 \mathrm{E}+02$ & $4.44 \mathrm{E}+00$ & $4.33 \mathrm{E}+02$ & $4.40 \mathrm{E}-06$ & 4.24E-06 & $4.35 \mathrm{E}-06$ & $5.85 \mathrm{E}-06$ & $9.38 \mathrm{E}-06$ \\
\hline 23 & $9.45 \mathrm{E}+02$ & $6.01 \mathrm{E}+00$ & $4.30 \mathrm{E}+02$ & $4.97 \mathrm{E}-06$ & $4.86 \mathrm{E}-06$ & $5.05 \mathrm{E}-06$ & $6.58 \mathrm{E}-06$ & $1.09 \mathrm{E}-05$ \\
\hline 24 & $8.67 \mathrm{E}+02$ & $8.40 \mathrm{E}+00$ & $3.82 \mathrm{E}+02$ & $5.95 \mathrm{E}-06$ & $5.85 \mathrm{E}-06$ & $6.03 \mathrm{E}-06$ & $7.78 \mathrm{E}-06$ & $1.36 \mathrm{E}-05$ \\
\hline 25 & $7.94 \mathrm{E}+02$ & $9.00 \mathrm{E}+00$ & $3.88 \mathrm{E}+02$ & 7.03E-06 & $6.95 \mathrm{E}-06$ & 7.17E-06 & $9.09 \mathrm{E}-06$ & $1.61 \mathrm{E}-05$ \\
\hline $26^{*}$ & $6.76 \mathrm{E}+02$ & $6.07 \mathrm{E}+00$ & $2.93 \mathrm{E}+02$ & $6.53 \mathrm{E}-06$ & $6.42 \mathrm{E}-06$ & $6.56 \mathrm{E}-06$ & $8.47 \mathrm{E}-06$ & $1.50 \mathrm{E}-05$ \\
\hline 27 & $5.57 \mathrm{E}+02$ & $7.54 \mathrm{E}+00$ & $3.06 \mathrm{E}+02$ & $9.25 \mathrm{E}-06$ & $9.20 \mathrm{E}-06$ & $9.34 \mathrm{E}-06$ & $1.15 \mathrm{E}-05$ & $2.16 \mathrm{E}-05$ \\
\hline 28 & $4.88 \mathrm{E}+02$ & $6.19 \mathrm{E}+00$ & $2.31 \mathrm{E}+02$ & $8.58 \mathrm{E}-06$ & $8.50 \mathrm{E}-06$ & $8.59 \mathrm{E}-06$ & $1.07 \mathrm{E}-05$ & $1.99 \mathrm{E}-05$ \\
\hline 29 & $4.11 \mathrm{E}+02$ & $4.24 \mathrm{E}+00$ & $1.85 \mathrm{E}+02$ & $8.43 \mathrm{E}-06$ & 8.32E-06 & 8.39E-06 & $1.05 \mathrm{E}-05$ & $1.94 \mathrm{E}-05$ \\
\hline 30 & $3.18 \mathrm{E}+02$ & $7.39 \mathrm{E}+00$ & $2.08 \mathrm{E}+02$ & $1.25 \mathrm{E}-05$ & $1.25 \mathrm{E}-05$ & $1.24 \mathrm{E}-05$ & $1.47 \mathrm{E}-05$ & $2.94 \mathrm{E}-05$ \\
\hline 31 & $2.62 \mathrm{E}+02$ & $1.75 \mathrm{E}+01$ & $2.66 \mathrm{E}+02$ & $1.43 \mathrm{E}-05$ & $1.42 \mathrm{E}-05$ & $1.40 \mathrm{E}-05$ & $1.67 \mathrm{E}-05$ & $3.60 \mathrm{E}-05$ \\
\hline 32 & $2.24 \mathrm{E}+02$ & $1.88 \mathrm{E}+01$ & $2.81 \mathrm{E}+02$ & $1.45 \mathrm{E}-05$ & $1.44 \mathrm{E}-05$ & $1.42 \mathrm{E}-05$ & $1.70 \mathrm{E}-05$ & $3.72 \mathrm{E}-05$ \\
\hline 33 & $1.61 \mathrm{E}+02$ & $1.41 \mathrm{E}+01$ & $2.77 \mathrm{E}+01$ & $1.43 \mathrm{E}-05$ & $1.42 \mathrm{E}-05$ & $1.40 \mathrm{E}-05$ & $1.69 \mathrm{E}-05$ & $3.64 \mathrm{E}-05$ \\
\hline 34 & $1.04 \mathrm{E}+02$ & $8.37 \mathrm{E}+01$ & $9.12 \mathrm{E}+02$ & $1.48 \mathrm{E}-05$ & $1.46 \mathrm{E}-05$ & $1.44 \mathrm{E}-05$ & $1.76 \mathrm{E}-05$ & $3.88 \mathrm{E}-05$ \\
\hline 35 & $8.22 \mathrm{E}+01$ & $1.19 \mathrm{E}+02$ & $1.08 \mathrm{E}+03$ & $1.47 \mathrm{E}-05$ & $1.46 \mathrm{E}-05$ & $1.44 \mathrm{E}-05$ & $1.75 \mathrm{E}-05$ & $3.86 \mathrm{E}-05$ \\
\hline
\end{tabular}

Note: * Best individual found with the Utopian Point Method

Table B.5: Pareto front individuals for the single-TMD control devices under the One-minus cosine gust profile excitation, for the operational cases 6 to 10 


\begin{tabular}{|c|c|c|c|c|c|c|c|c|}
\hline \multirow{2}{*}{ Pareto Point ID } & \multirow{2}{*}{ m (lb) } & \multirow{2}{*}{ c (lbf.s/in) } & \multirow{2}{*}{ k (lbf/in) } & \multicolumn{5}{|c|}{$U_{\text {fitness }}[\mathrm{in}]$} \\
\hline & & & & Case 6 & Case 7 & Case 8 & Case 9 & Case 10 \\
\hline 1 & $2.43 \mathrm{E}+03$ & $2.74 \mathrm{E}+00$ & $9.86 \mathrm{E}+02$ & 7.39E-06 & $1.19 \mathrm{E}-05$ & $2.26 \mathrm{E}-05$ & $6.59 \mathrm{E}-05$ & $1.82 \mathrm{E}-04$ \\
\hline 2 & $2.40 \mathrm{E}+03$ & $6.78 \mathrm{E}+00$ & $1.03 \mathrm{E}+03$ & $6.04 \mathrm{E}-06$ & $1.05 \mathrm{E}-05$ & $2.15 \mathrm{E}-05$ & $6.11 \mathrm{E}-05$ & $1.98 \mathrm{E}-04$ \\
\hline 3 & $2.32 \mathrm{E}+03$ & $5.51 \mathrm{E}+00$ & $9.93 \mathrm{E}+02$ & $6.28 \mathrm{E}-06$ & $1.07 \mathrm{E}-05$ & $2.14 \mathrm{E}-05$ & $6.06 \mathrm{E}-05$ & $1.88 \mathrm{E}-04$ \\
\hline 4 & $2.30 \mathrm{E}+03$ & $2.52 \mathrm{E}+01$ & $6.97 \mathrm{E}+02$ & 4.53E-06 & $9.79 \mathrm{E}-06$ & $2.18 \mathrm{E}-05$ & $8.12 \mathrm{E}-05$ & $2.50 \mathrm{E}-04$ \\
\hline 5 & $2.26 \mathrm{E}+03$ & $5.29 \mathrm{E}+00$ & $9.60 \mathrm{E}+02$ & $6.51 \mathrm{E}-06$ & $1.10 \mathrm{E}-05$ & $2.17 \mathrm{E}-05$ & $6.26 \mathrm{E}-05$ & $1.92 \mathrm{E}-04$ \\
\hline 6 & $2.15 \mathrm{E}+03$ & $5.29 \mathrm{E}+00$ & $9.57 \mathrm{E}+02$ & $6.21 \mathrm{E}-06$ & $1.13 \mathrm{E}-05$ & $2.24 \mathrm{E}-05$ & $6.74 \mathrm{E}-05$ & $2.15 \mathrm{E}-04$ \\
\hline 7 & $2.09 \mathrm{E}+03$ & $5.22 \mathrm{E}+00$ & $8.91 \mathrm{E}+02$ & $6.92 \mathrm{E}-06$ & $1.16 \mathrm{E}-05$ & $2.27 \mathrm{E}-05$ & $6.87 \mathrm{E}-05$ & $2.08 \mathrm{E}-04$ \\
\hline 8 & $1.96 \mathrm{E}+03$ & $7.35 \mathrm{E}+00$ & $9.48 \mathrm{E}+02$ & $6.77 \mathrm{E}-06$ & $1.56 \mathrm{E}-05$ & $3.36 \mathrm{E}-05$ & $1.03 \mathrm{E}-04$ & 4.00E-04 \\
\hline 9 & $1.94 \mathrm{E}+03$ & $6.22 \mathrm{E}+00$ & $8.54 \mathrm{E}+02$ & $6.95 \mathrm{E}-06$ & $1.24 \mathrm{E}-05$ & $2.51 \mathrm{E}-05$ & $7.88 \mathrm{E}-05$ & $2.61 \mathrm{E}-04$ \\
\hline 10 & $1.83 \mathrm{E}+03$ & $6.33 \mathrm{E}+00$ & $8.41 \mathrm{E}+02$ & 7.03E-06 & $1.39 \mathrm{E}-05$ & $2.90 \mathrm{E}-05$ & $9.13 \mathrm{E}-05$ & $3.29 \mathrm{E}-04$ \\
\hline 11 & $1.82 \mathrm{E}+03$ & $5.52 \mathrm{E}+00$ & $7.88 \mathrm{E}+02$ & 7.61E-06 & $1.28 \mathrm{E}-05$ & $2.53 \mathrm{E}-05$ & $8.16 \mathrm{E}-05$ & $2.57 \mathrm{E}-04$ \\
\hline 12 & $1.74 \mathrm{E}+03$ & $4.48 \mathrm{E}+00$ & $7.49 \mathrm{E}+02$ & $8.04 \mathrm{E}-06$ & $1.32 \mathrm{E}-05$ & $2.49 \mathrm{E}-05$ & 8.34E-05 & $2.45 \mathrm{E}-04$ \\
\hline 13 & $1.64 \mathrm{E}+03$ & $7.81 \mathrm{E}+00$ & $7.82 \mathrm{E}+02$ & $8.16 \mathrm{E}-06$ & $1.83 \mathrm{E}-05$ & 4.07E-05 & $1.24 \mathrm{E}-04$ & 4.95E-04 \\
\hline 14 & $1.58 \mathrm{E}+03$ & $7.35 \mathrm{E}+00$ & $7.33 \mathrm{E}+02$ & $8.32 \mathrm{E}-06$ & $1.73 \mathrm{E}-05$ & $3.80 \mathrm{E}-05$ & $1.17 \mathrm{E}-04$ & $4.55 \mathrm{E}-04$ \\
\hline 15 & $1.53 \mathrm{E}+03$ & $7.34 \mathrm{E}+00$ & $6.89 \mathrm{E}+02$ & $8.70 \mathrm{E}-06$ & $1.68 \mathrm{E}-05$ & $3.70 \mathrm{E}-05$ & $1.15 \mathrm{E}-04$ & $4.37 \mathrm{E}-04$ \\
\hline 16 & $1.40 \mathrm{E}+03$ & $8.48 \mathrm{E}+00$ & $5.88 \mathrm{E}+02$ & $1.06 \mathrm{E}-05$ & $1.88 \mathrm{E}-05$ & 4.29E-05 & $1.29 \mathrm{E}-04$ & $5.19 \mathrm{E}-04$ \\
\hline 17 & $1.33 \mathrm{E}+03$ & $8.51 \mathrm{E}+00$ & $6.39 \mathrm{E}+02$ & $1.04 \mathrm{E}-05$ & $2.40 \mathrm{E}-05$ & $5.49 \mathrm{E}-05$ & $1.66 \mathrm{E}-04$ & $6.95 \mathrm{E}-04$ \\
\hline 18 & $1.25 \mathrm{E}+03$ & $4.77 \mathrm{E}+00$ & $5.42 \mathrm{E}+02$ & $1.07 \mathrm{E}-05$ & $1.79 \mathrm{E}-05$ & $3.66 \mathrm{E}-05$ & $1.22 \mathrm{E}-04$ & $4.22 \mathrm{E}-04$ \\
\hline 19 & $1.18 \mathrm{E}+03$ & $8.87 \mathrm{E}+00$ & $5.78 \mathrm{E}+02$ & $1.20 \mathrm{E}-05$ & $2.78 \mathrm{E}-05$ & $6.50 \mathrm{E}-05$ & $1.95 \mathrm{E}-04$ & $8.41 \mathrm{E}-04$ \\
\hline 20 & $1.09 \mathrm{E}+03$ & $5.27 \mathrm{E}+00$ & $4.72 \mathrm{E}+02$ & $1.21 \mathrm{E}-05$ & $2.06 \mathrm{E}-05$ & $4.52 \mathrm{E}-05$ & $1.43 \mathrm{E}-04$ & $5.44 \mathrm{E}-04$ \\
\hline 21 & $1.05 \mathrm{E}+03$ & $4.86 \mathrm{E}+00$ & $4.18 \mathrm{E}+02$ & $1.45 \mathrm{E}-05$ & $2.18 \mathrm{E}-05$ & 4.74E-05 & $1.52 \mathrm{E}-04$ & $5.58 \mathrm{E}-04$ \\
\hline 22 & $9.74 \mathrm{E}+02$ & $4.44 \mathrm{E}+00$ & $4.33 \mathrm{E}+02$ & $1.28 \mathrm{E}-05$ & $2.27 \mathrm{E}-05$ & 4.94E-05 & $1.58 \mathrm{E}-04$ & $6.06 \mathrm{E}-04$ \\
\hline 23 & $9.45 \mathrm{E}+02$ & $6.01 \mathrm{E}+00$ & $4.30 \mathrm{E}+02$ & $1.33 \mathrm{E}-05$ & $2.61 \mathrm{E}-05$ & $6.08 \mathrm{E}-05$ & $1.84 \mathrm{E}-04$ & 7.73E-04 \\
\hline 24 & $8.67 \mathrm{E}+02$ & $8.40 \mathrm{E}+00$ & $3.82 \mathrm{E}+02$ & $1.59 \mathrm{E}-05$ & $3.00 \mathrm{E}-05$ & 7.33E-05 & $2.14 \mathrm{E}-04$ & $9.48 \mathrm{E}-04$ \\
\hline 25 & $7.94 \mathrm{E}+02$ & $9.00 \mathrm{E}+00$ & $3.88 \mathrm{E}+02$ & $1.74 \mathrm{E}-05$ & $3.58 \mathrm{E}-05$ & $8.80 \mathrm{E}-05$ & $2.58 \mathrm{E}-04$ & $1.18 \mathrm{E}-03$ \\
\hline $26^{*}$ & $6.76 \mathrm{E}+02$ & $6.07 \mathrm{E}+00$ & $2.93 \mathrm{E}+02$ & $1.82 \mathrm{E}-05$ & $3.20 \mathrm{E}-05$ & $7.78 \mathrm{E}-05$ & $2.28 \mathrm{E}-04$ & $1.02 \mathrm{E}-03$ \\
\hline
\end{tabular}




\begin{tabular}{lllllllll}
27 & $5.57 \mathrm{E}+02$ & $7.54 \mathrm{E}+00$ & $3.06 \mathrm{E}+02$ & $2.24 \mathrm{E}-05$ & $4.28 \mathrm{E}-05$ & $1.09 \mathrm{E}-04$ & $3.09 \mathrm{E}-04$ & $1.50 \mathrm{E}-03$ \\
28 & $4.88 \mathrm{E}+02$ & $6.19 \mathrm{E}+00$ & $2.31 \mathrm{E}+02$ & $2.25 \mathrm{E}-05$ & $3.92 \mathrm{E}-05$ & $9.86 \mathrm{E}-05$ & $2.82 \mathrm{E}-04$ & $1.34 \mathrm{E}-03$ \\
29 & $4.11 \mathrm{E}+02$ & $4.24 \mathrm{E}+00$ & $1.85 \mathrm{E}+02$ & $2.33 \mathrm{E}-05$ & $3.80 \mathrm{E}-05$ & $9.42 \mathrm{E}-05$ & $2.74 \mathrm{E}-04$ & $1.27 \mathrm{E}-03$ \\
30 & $3.18 \mathrm{E}+02$ & $7.39 \mathrm{E}+00$ & $2.08 \mathrm{E}+02$ & $3.30 \mathrm{E}-05$ & $4.57 \mathrm{E}-05$ & $1.21 \mathrm{E}-04$ & $3.30 \mathrm{E}-04$ & $1.62 \mathrm{E}-03$ \\
31 & $2.62 \mathrm{E}+02$ & $1.75 \mathrm{E}+01$ & $2.66 \mathrm{E}+02$ & $4.30 \mathrm{E}-05$ & $4.80 \mathrm{E}-05$ & $1.32 \mathrm{E}-04$ & $3.52 \mathrm{E}-04$ & $1.69 \mathrm{E}-03$ \\
32 & $2.24 \mathrm{E}+02$ & $1.88 \mathrm{E}+01$ & $2.81 \mathrm{E}+02$ & $4.51 \mathrm{E}-05$ & $4.84 \mathrm{E}-05$ & $1.33 \mathrm{E}-04$ & $3.58 \mathrm{E}-04$ & $1.69 \mathrm{E}-03$ \\
33 & $1.61 \mathrm{E}+02$ & $1.41 \mathrm{E}+01$ & $2.77 \mathrm{E}+01$ & $4.53 \mathrm{E}-05$ & $4.79 \mathrm{E}-05$ & $1.29 \mathrm{E}-04$ & $3.59 \mathrm{E}-04$ & $1.63 \mathrm{E}-03$ \\
34 & $1.04 \mathrm{E}+02$ & $8.37 \mathrm{E}+01$ & $9.12 \mathrm{E}+02$ & $4.90 \mathrm{E}-05$ & $4.93 \mathrm{E}-05$ & $1.34 \mathrm{E}-04$ & $3.74 \mathrm{E}-04$ & $1.65 \mathrm{E}-03$ \\
35 & $8.22 \mathrm{E}+01$ & $1.19 \mathrm{E}+02$ & $1.08 \mathrm{E}+03$ & $4.90 \mathrm{E}-05$ & $4.93 \mathrm{E}-05$ & $1.33 \mathrm{E}-04$ & $3.74 \mathrm{E}-04$ & $1.64 \mathrm{E}-03$ \\
\hline
\end{tabular}

Note: * Best individual found with the Utopian Point Method

Table B.6: Pareto front individuals for the single-TMD control devices under the One-minus cosine gust profile excitation, for the operational cases 11 to 14

\begin{tabular}{|c|c|c|c|c|c|c|c|}
\hline \multirow{2}{*}{ Pareto Point ID } & \multirow{2}{*}{ m (lb) } & \multirow{2}{*}{ c (lbf.s/in) } & \multirow{2}{*}{ k (lbf/in) } & \multicolumn{4}{|c|}{$U_{\text {fitness }}[\mathrm{in}]$} \\
\hline & & & & Case 11 & Case 12 & Case 13 & Case 14 \\
\hline 1 & $2.43 \mathrm{E}+03$ & $2.74 \mathrm{E}+00$ & $9.86 \mathrm{E}+02$ & $6.22 \mathrm{E}-04$ & 1.19E-03 & $2.39 \mathrm{E}-03$ & 7.27E-03 \\
\hline 2 & $2.40 \mathrm{E}+03$ & $6.78 \mathrm{E}+00$ & $1.03 \mathrm{E}+03$ & 4.99E-04 & $9.98 \mathrm{E}-04$ & $2.28 \mathrm{E}-03$ & $6.25 \mathrm{E}-03$ \\
\hline 3 & $2.32 \mathrm{E}+03$ & $5.51 \mathrm{E}+00$ & $9.93 \mathrm{E}+02$ & $5.14 \mathrm{E}-04$ & $1.04 \mathrm{E}-03$ & $2.24 \mathrm{E}-03$ & $6.27 \mathrm{E}-03$ \\
\hline 4 & $2.30 \mathrm{E}+03$ & $2.52 \mathrm{E}+01$ & $6.97 \mathrm{E}+02$ & $2.71 \mathrm{E}-04$ & $3.76 \mathrm{E}-04$ & $1.83 \mathrm{E}-03$ & 4.63E-03 \\
\hline 5 & $2.26 \mathrm{E}+03$ & $5.29 \mathrm{E}+00$ & $9.60 \mathrm{E}+02$ & $5.34 \mathrm{E}-04$ & $1.08 \mathrm{E}-03$ & $2.29 \mathrm{E}-03$ & $6.46 \mathrm{E}-03$ \\
\hline 6 & $2.15 \mathrm{E}+03$ & $5.29 \mathrm{E}+00$ & $9.57 \mathrm{E}+02$ & $4.91 \mathrm{E}-04$ & $1.03 \mathrm{E}-03$ & $2.43 \mathrm{E}-03$ & $6.34 \mathrm{E}-03$ \\
\hline 7 & $2.09 \mathrm{E}+03$ & $5.22 \mathrm{E}+00$ & $8.91 \mathrm{E}+02$ & $5.65 \mathrm{E}-04$ & $1.15 \mathrm{E}-03$ & $2.44 \mathrm{E}-03$ & $6.87 \mathrm{E}-03$ \\
\hline 8 & $1.96 \mathrm{E}+03$ & $7.35 \mathrm{E}+00$ & $9.48 \mathrm{E}+02$ & $5.93 \mathrm{E}-04$ & $1.11 \mathrm{E}-03$ & $4.15 \mathrm{E}-03$ & $9.37 \mathrm{E}-03$ \\
\hline 9 & $1.94 \mathrm{E}+03$ & $6.22 \mathrm{E}+00$ & $8.54 \mathrm{E}+02$ & $5.70 \mathrm{E}-04$ & $1.15 \mathrm{E}-03$ & $2.84 \mathrm{E}-03$ & 7.47E-03 \\
\hline 10 & $1.83 \mathrm{E}+03$ & $6.33 \mathrm{E}+00$ & $8.41 \mathrm{E}+02$ & $5.82 \mathrm{E}-04$ & $1.17 \mathrm{E}-03$ & $3.47 \mathrm{E}-03$ & 8.37E-03 \\
\hline 11 & $1.82 \mathrm{E}+03$ & $5.52 \mathrm{E}+00$ & $7.88 \mathrm{E}+02$ & $6.22 \mathrm{E}-04$ & $1.26 \mathrm{E}-03$ & $2.87 \mathrm{E}-03$ & $7.82 \mathrm{E}-03$ \\
\hline 12 & $1.74 \mathrm{E}+03$ & $4.48 \mathrm{E}+00$ & $7.49 \mathrm{E}+02$ & $6.43 \mathrm{E}-04$ & $1.34 \mathrm{E}-03$ & $2.82 \mathrm{E}-03$ & $7.81 \mathrm{E}-03$ \\
\hline
\end{tabular}




\begin{tabular}{|c|c|c|c|c|c|c|c|}
\hline 13 & $1.64 \mathrm{E}+03$ & $7.81 \mathrm{E}+00$ & $7.82 \mathrm{E}+02$ & 7.62E-04 & $1.35 \mathrm{E}-03$ & $5.10 \mathrm{E}-03$ & $1.19 \mathrm{E}-02$ \\
\hline 14 & $1.58 \mathrm{E}+03$ & $7.35 \mathrm{E}+00$ & $7.33 \mathrm{E}+02$ & $7.52 \mathrm{E}-04$ & $1.38 \mathrm{E}-03$ & 4.73E-03 & $1.14 \mathrm{E}-02$ \\
\hline 15 & $1.53 \mathrm{E}+03$ & $7.34 \mathrm{E}+00$ & $6.89 \mathrm{E}+02$ & $7.80 \mathrm{E}-04$ & $1.44 \mathrm{E}-03$ & $4.58 \mathrm{E}-03$ & $1.13 \mathrm{E}-02$ \\
\hline 16 & $1.40 \mathrm{E}+03$ & $8.48 \mathrm{E}+00$ & $5.88 \mathrm{E}+02$ & $1.00 \mathrm{E}-03$ & $1.72 \mathrm{E}-03$ & $5.46 \mathrm{E}-03$ & $1.41 \mathrm{E}-02$ \\
\hline 17 & $1.33 \mathrm{E}+03$ & $8.51 \mathrm{E}+00$ & $6.39 \mathrm{E}+02$ & $1.08 \mathrm{E}-03$ & $1.73 \mathrm{E}-03$ & $7.29 \mathrm{E}-03$ & $1.72 \mathrm{E}-02$ \\
\hline 18 & $1.25 \mathrm{E}+03$ & $4.77 \mathrm{E}+00$ & $5.42 \mathrm{E}+02$ & 8.95E-04 & $1.78 \mathrm{E}-03$ & $4.51 \mathrm{E}-03$ & $1.17 \mathrm{E}-02$ \\
\hline 19 & $1.18 \mathrm{E}+03$ & $8.87 \mathrm{E}+00$ & $5.78 \mathrm{E}+02$ & $1.30 \mathrm{E}-03$ & $1.98 \mathrm{E}-03$ & 8.97E-03 & $2.12 \mathrm{E}-02$ \\
\hline 20 & $1.09 \mathrm{E}+03$ & $5.27 \mathrm{E}+00$ & $4.72 \mathrm{E}+02$ & $1.08 \mathrm{E}-03$ & $1.99 \mathrm{E}-03$ & $5.77 \mathrm{E}-03$ & $1.48 \mathrm{E}-02$ \\
\hline 21 & $1.05 \mathrm{E}+03$ & $4.86 \mathrm{E}+00$ & $4.18 \mathrm{E}+02$ & $1.28 \mathrm{E}-03$ & $2.34 \mathrm{E}-03$ & $6.03 \mathrm{E}-03$ & $1.65 \mathrm{E}-02$ \\
\hline 22 & $9.74 \mathrm{E}+02$ & $4.44 \mathrm{E}+00$ & $4.33 \mathrm{E}+02$ & $1.13 \mathrm{E}-03$ & $2.12 \mathrm{E}-03$ & $6.44 \mathrm{E}-03$ & $1.61 \mathrm{E}-02$ \\
\hline 23 & $9.45 \mathrm{E}+02$ & $6.01 \mathrm{E}+00$ & $4.30 \mathrm{E}+02$ & $1.33 \mathrm{E}-03$ & $2.21 \mathrm{E}-03$ & $8.29 \mathrm{E}-03$ & $2.04 \mathrm{E}-02$ \\
\hline 24 & $8.67 \mathrm{E}+02$ & $8.40 \mathrm{E}+00$ & $3.82 \mathrm{E}+02$ & $1.71 \mathrm{E}-03$ & $2.58 \mathrm{E}-03$ & $1.05 \mathrm{E}-02$ & $2.67 \mathrm{E}-02$ \\
\hline 25 & $7.94 \mathrm{E}+02$ & $9.00 \mathrm{E}+00$ & $3.88 \mathrm{E}+02$ & $2.02 \mathrm{E}-03$ & $2.83 \mathrm{E}-03$ & $1.34 \mathrm{E}-02$ & $3.36 \mathrm{E}-02$ \\
\hline $26^{*}$ & $6.76 \mathrm{E}+02$ & $6.07 \mathrm{E}+00$ & $2.93 \mathrm{E}+02$ & $1.89 \mathrm{E}-03$ & $2.92 \mathrm{E}-03$ & $1.14 \mathrm{E}-02$ & $2.92 \mathrm{E}-02$ \\
\hline 27 & $5.57 \mathrm{E}+02$ & $7.54 \mathrm{E}+00$ & $3.06 \mathrm{E}+02$ & $2.71 \mathrm{E}-03$ & $3.44 \mathrm{E}-03$ & $1.80 \mathrm{E}-02$ & 4.63E-02 \\
\hline 28 & $4.88 \mathrm{E}+02$ & $6.19 \mathrm{E}+00$ & $2.31 \mathrm{E}+02$ & $2.52 \mathrm{E}-03$ & $3.53 \mathrm{E}-03$ & $1.58 \mathrm{E}-02$ & 4.09E-02 \\
\hline 29 & $4.11 \mathrm{E}+02$ & $4.24 \mathrm{E}+00$ & $1.85 \mathrm{E}+02$ & $2.46 \mathrm{E}-03$ & $3.65 \mathrm{E}-03$ & $1.48 \mathrm{E}-02$ & $3.87 \mathrm{E}-02$ \\
\hline 30 & $3.18 \mathrm{E}+02$ & $7.39 \mathrm{E}+00$ & $2.08 \mathrm{E}+02$ & $3.54 \mathrm{E}-03$ & $4.52 \mathrm{E}-03$ & $1.95 \mathrm{E}-02$ & $5.37 \mathrm{E}-02$ \\
\hline 31 & $2.62 \mathrm{E}+02$ & $1.75 \mathrm{E}+01$ & $2.66 \mathrm{E}+02$ & 4.32E-03 & $5.52 \mathrm{E}-03$ & $1.99 \mathrm{E}-02$ & $5.70 \mathrm{E}-02$ \\
\hline 32 & $2.24 \mathrm{E}+02$ & $1.88 \mathrm{E}+01$ & $2.81 \mathrm{E}+02$ & $4.46 \mathrm{E}-03$ & $5.78 \mathrm{E}-03$ & $1.97 \mathrm{E}-02$ & $5.73 \mathrm{E}-02$ \\
\hline 33 & $1.61 \mathrm{E}+02$ & $1.41 \mathrm{E}+01$ & $2.77 \mathrm{E}+01$ & $4.38 \mathrm{E}-03$ & $5.91 \mathrm{E}-03$ & $1.90 \mathrm{E}-02$ & $5.54 \mathrm{E}-02$ \\
\hline 34 & $1.04 \mathrm{E}+02$ & $8.37 \mathrm{E}+01$ & $9.12 \mathrm{E}+02$ & $4.65 \mathrm{E}-03$ & $6.34 \mathrm{E}-03$ & $1.90 \mathrm{E}-02$ & $5.67 \mathrm{E}-02$ \\
\hline 35 & $8.22 \mathrm{E}+01$ & $1.19 \mathrm{E}+02$ & $1.08 \mathrm{E}+03$ & $4.63 \mathrm{E}-03$ & $6.37 \mathrm{E}-03$ & $1.89 \mathrm{E}-02$ & $5.63 \mathrm{E}-02$ \\
\hline
\end{tabular}

Note: * Best individual found with the Utopian Point Method 


\section{References}

[1] M.G. Nieto, P. V. Thomas, M.S.A. ElSayed, M. Saad, G.L. Brown, L.M. Hilliard, Development of Efficient Dynamic Aeroelasticity Model for High Fidelity Pointing Accuracy Assessment of VLBI Earth-Based Radio Antennas, Int. J. Aeronaut. Sp. Sci. 21 (2020) 693-706. https://doi.org/10.1007/s42405-019-00238-6.

[2] J. Zhang, J. Huang, W. Liang, Y. Zhang, Q. Xu, L. Yi, C. Wang, A Correction Method of Estimating the Pointing Error for Reflector Antenna, Shock Vib. 2018 (2018). https://doi.org/10.1155/2018/3262869.

[3] S. Raja, Novel Aeroelastic Approaches for Transonic Flutter and Buffet Clearance of Aerospace Vehicles, Trans. Indian Natl. Acad. Eng. (2021). https://doi.org/10.1007/s41403020-00190-y.

[4] M.H. Hansen, Aeroelastic instability problems for wind turbines, Wind Energy. 10 (2007) 551-577. https://doi.org/10.1002/we.242.

[5] D. Rajpal, C. Kassapoglou, R. De Breuker, Aeroelastic optimization of composite wings including fatigue loading requirements, Compos. Struct. 227 (2019) 111248. https://doi.org/10.1016/j.compstruct.2019.111248.

[6] S.M. Srivatsa, D.J. Inman, Characterization of Fatigue in Integrated Tuned Mass-Dampers BT - Special Topics in Structural Dynamics \& Experimental Techniques, Volume 5, in: D.S. Epp (Ed.), Springer International Publishing, Cham, 2021: pp. 25-35.

[7] H. V. de Figueiredo, D.F. Castillo-Zúñiga, N.C. Costa, O. Saotome, R.G.A. da Silva, Aeroelastic Vibration Measurement Based on Laser and Computer Vision Technique, Exp. Tech. 45 (2021) 95-107. https://doi.org/10.1007/s40799-020-00399-0.

[8] M. Arena, A. Chiariello, M. Castaldo, L. Di Palma, Vibration Response Aspects of a Main 
Landing Gear Composite Door Designed for High-Speed Rotorcraft, Aerospace. 8 (2021) 52. https://doi.org/10.3390/aerospace8020052.

[9] S. Nima Mahmoodi, M. Ahmadian, Active vibration control with modified positive position feedback, J. Dyn. Syst. Meas. Control. Trans. ASME. 131 (2009) 1-8. https://doi.org/10.1115/1.3089565.

[10] A.J. Keane, A.P. Bright, Passive vibration control via unusual geometries: Experiments on model aerospace structures, J. Sound Vib. 190 (1996) 713-719. https://doi.org/10.1006/jsvi.1996.0086.

[11] S.K. Kwak, G. Washington, R.K. Yedavalli, Active and passive vibration control of landing gear components, Am. Soc. Mech. Eng. Aerosp. Div. AD. 59 (1999) 269-275. https://doi.org/10.1061/(ASCE)0893-1321(2002)15.

[12] T.P. Sales, D.A. Rade, L.C.G. De Souza, Passive vibration control of flexible spacecraft using shunted piezoelectric transducers, Aerosp. Sci. Technol. 29 (2013) 403-412. https://doi.org/10.1016/j.ast.2013.05.001.

[13] G.M. Chatziathanasiou, N.A. Chrysochoidis, K.I. Georgopoulos-Bosinas, D.A. Saravanos, Semi-active vibration control of aircraft structures, AIAA Scitech 2021 Forum. (2021) 110. https://doi.org/10.2514/6.2021-1736.

[14] D. Karnopp, M.J. Crosby, R.A. Harwood, Vibration Control Using Semi-Active Force Generators., ASME Pap. (1973) 619-626.

[15] S. Gao, J. Liu, Adaptive fault-tolerant boundary vibration control for a flexible aircraft wing against actuator and sensor faults, JVC/Journal Vib. Control. (2021). https://doi.org/10.1177/1077546320986715.

[16] L. Zhang, S. Xu, Z. Zhang, N. Cui, Active vibration suppression for flexible satellites using 
a novel component synthesis method, Adv. Sp. Res. 67 (2021) 1968-1980. https://doi.org/10.1016/j.asr.2020.12.028.

[17] M.V.G. de Morais, A.A.O. Lopez, J.F. Martins, L.J. Pedroso, Equivalent mechanical model of rectangular container attached to a pendulum compared to experimental data and analytical solution, J. Brazilian Soc. Mech. Sci. Eng. 42 (2020) 1-10. https://doi.org/10.1007/s40430-020-2232-7.

[18] W. Wang, Z. Yang, X. Hua, Z. Chen, X. Wang, G. Song, Evaluation of a pendulum pounding tuned mass damper for seismic control of structures, Eng. Struct. 228 (2021) 111554. https://doi.org/10.1016/j.engstruct.2020.111554.

[19] J.L. Almazán, J.C. De la Llera, J.A. Inaudi, D. López-García, L.E. Izquierdo, A bidirectional and homogeneous tuned mass damper: A new device for passive control of vibrations, Eng. Struct. 29 (2007) 1548-1560. https://doi.org/10.1016/j.engstruct.2006.09.005.

[20] M. Gutierrez Soto, H. Adeli, Tuned Mass Dampers, Arch. Comput. Methods Eng. 20 (2013) 419-431. https://doi.org/10.1007/s11831-013-9091-7.

[21] F. Ogilvy, How Renault F1 won a World championship by creating the tuned mass damper, Moore Good Ink. (n.d.). https://mooregoodink.com/how-renault-f1-found-faster-lap-timesand-won-a-world-championship-by-creating-the-tuned-mass-damper/ (accessed June 30, 2021).

[22] S. Elias, V. Matsagar, Distributed Multiple Tuned Mass Dampers for Wind Vibration Response Control of High-Rise Building, J. Eng. (United Kingdom). 2014 (2014). https://doi.org/10.1155/2014/198719.

[23] L. Suresh, K.M. Mini, Effect of Multiple Tuned Mass Dampers for Vibration Control in High-Rise Buildings, Pract. Period. Struct. Des. Constr. 24 (2019) 04019031. 
https://doi.org/10.1061/(asce)sc.1943-5576.0000453.

[24] L.S. Vellar, S.P. Ontiveros-Pérez, L.F.F. Miguel, L.F. Fadel Miguel, Robust Optimum Design of Multiple Tuned Mass Dampers for Vibration Control in Buildings Subjected to Seismic Excitation, Shock Vib. 2019 (2019). https://doi.org/10.1155/2019/9273714.

[25] N. Isyumov, A.G. Davenport, J. Monbaliu, CN Tower, Toronto: Model and full scale response to wind, (1984).

[26] B. Kolator, J. Pelc, Passive vibration damping in a truss telecommunication tower, Tech. Sci. / Univ. Warm. Maz. Olsztyn. 17 (2014) 249-258.

[27] Z. Zhang, T.G. Larsen, Optimal calibration of the rotational inertia double tuned mass damper (RIDTMD) for rotating wind turbine blades, J. Sound Vib. 493 (2021) 115827. https://doi.org/10.1016/j.jsv.2020.115827.

[28] D. Chen, S. Huang, C. Huang, R. Liu, F. Ouyang, Passive control of jacket-type offshore wind turbine vibrations by single and multiple tuned mass dampers, Mar. Struct. 77 (2021) 102938. https://doi.org/10.1016/j.marstruc.2021.102938.

[29] N. Debnath, S.K. Deb, A. Dutta, Multi-modal vibration control of truss bridges with tuned mass dampers under general loading, JVC/Journal Vib. Control. 22 (2016) 4121-4140. https://doi.org/10.1177/1077546315571172.

[30] Y. Daniel, O. Lavan, R. Levy, Multiple-Tuned Mass Dampers for Multimodal Control of

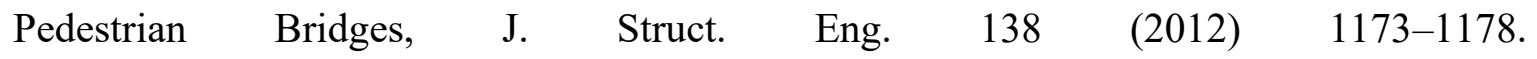
https://doi.org/10.1061/(asce)st.1943-541x.0000527.

[31] J.S. Hagelin, S.K. Ruegamer, T.D. Thornton, S.C. O’Meara, J.R. Ellis, J. E. Fred Gylland, Aeroelastic Tuned Mass Damper, US 2013/0092489 A1, 2013.

[32] Y.R. Lee, H.S. Kim, J.W. Kang, Seismic Response Control Performance Evaluation of 
Tuned Mass Dampers for a Retractable-Roof Spatial Structure, Int. J. Steel Struct. 21 (2021) 213-224. https://doi.org/10.1007/s13296-020-00431-4.

[33] Z. Liu, Y. Wang, X. Hua, H. Zhu, Z. Zhu, Optimization of wind turbine TMD under real wind distribution countering wake effects using GPU acceleration and machine learning technologies, J. Wind Eng. Ind. Aerodyn. $208 \quad$ (2021) 104436. https://doi.org/10.1016/j.jweia.2020.104436.

[34] W. Ma, J. Yu, Y. Yang, Graphical Design Methodology of Multi-Degrees-of-Freedom Tuned Mass Damper for Suppressing Multiple Modes, J. Vib. Acoust. 143 (2021) 1-12. https://doi.org/10.1115/1.4047860.

[35] Y. Zhang, J. Zhang, G. Zhai, Vibration isolation platform with multiple tuned mass dampers for reaction wheel on satellites, Math. Probl. Eng. 2013 (2013). https://doi.org/10.1155/2013/574072.

[36] F. Meng, J. Wan, Y. Xia, Y. Ma, J. Yu, A multi-degree of freedom tuned mass damper design for vibration mitigation of a suspension bridge, Appl. Sci. 10 (2020). https://doi.org/10.3390/app10020457.

[37] H. Yamaguchi, N. Harnpornchai, Fundamental characteristics of Multiple Tuned Mass Dampers for suppressing harmonically forced oscillations, Earthq. Eng. Struct. Dyn. 22 (1993) 51-62. https://doi.org/10.1002/eqe.4290220105.

[38] T.P. Boivin A, Boilard F, Messier PL, 12 m VLBI antenna structural simulation, 2014.

[39] E.M. Kasprzak, K.E. Lewis, Pareto analysis in multiobjective optimization using the collinearity theorem and scaling method, Struct. Multidiscip. Optim. 22 (2001) 208-218. https://doi.org/10.1007/s001580100138.

[40] A. Kareem, T. Kijewski, Y. Tamura, Mitigation of motions of tall buildings with specific 
examples of recent applications, Wind Struct. An Int. J. 2 (1999) 201-251. https://doi.org/10.12989/was.1999.2.3.201.

[41] C. Tsioustas, M. Loupis, Design, simulation and experimental verification of an active inductance for a low frequency tuned mass damper in an aircraft fuselage active vibration cancellation system, 2018 Int. Symp. Ind. Electron. INDEL 2018 - Proc. (2019) 4-8. https://doi.org/10.1109/INDEL.2018.8637606.

[42] J.J. Connor, Introduction to Structural Motion Control, Prentice Hall Pearson Education, Incorporated, 2003. https://books.google.ca/books?id=te1RAAAAMAAJ.

[43] D.J. Inman, Engineering Vibration, Prentice Hall, 2001. https://books.google.ca/books?id=bHIeAQAAIAAJ.

[44] G. Bertollucci Colherinhas, F. Petrini, M.V.G. de Morais, F. Bontempi, Optimal design of passive-adaptive pendulum tuned mass damper for the global vibration control of offshore wind turbines, Wind Energy. (2020). https://doi.org/10.1002/we.2590.

[45] R. Lewandowski, J. Grzymisławska, Dynamic analysis of structures with multiple tuned mass dampers, J. Civ. Eng. Manag. 15 (2009) 77-86. https://doi.org/10.3846/13923730.2009.15.77-86.

[46] M. Setareh, J.K. Ritchey, A.J. Baxter, T.M. Murray, Pendulum Tuned Mass Dampers for Floor Vibration Control, J. Perform. Constr. Facil. 20 (2006) 64-73. https://doi.org/10.1061/(asce)0887-3828(2006)20:1(64).

[47] Z. Wang, H. Gao, H. Wang, Z. Chen, Development of stiffness-adjustable tuned mass dampers for frequency retuning, Adv. Struct. Eng. 22 (2019) 473-485. https://doi.org/10.1177/1369433218791356.

[48] S. Elias, V. Matsagar, Research developments in vibration control of structures using 
passive tuned mass dampers, Annu. Rev. Control. 44 (2017) 129-156. https://doi.org/10.1016/j.arcontrol.2017.09.015.

[49] Hermann Frahm, Device for damping vibrations of bodies, US989958A, 1909. https://patents.google.com/patent/US989958A/en.

[50] Z. Zhang, C. Høeg, Inerter-enhanced tuned mass damper for vibration damping of floating offshore wind turbines, Ocean Eng. $223 \quad$ (2021) 1-18. https://doi.org/10.1016/j.oceaneng.2021.108663.

[51] J.O. Martín del Campo, A. Pozos-Estrada, O. Pozos-Estrada, Development of fragility curves of land-based wind turbines with tuned mass dampers under cyclone and seismic loading, Wind Energy. 24 (2021) 737-753. https://doi.org/10.1002/we.2600.

[52] V. Boonyapinyo, A. Aksorn, P. Lukkunaprasit, Suppression of aerodynamic response of suspension bridges during erection and after completion by using tuned mass dampers, Wind Struct. 10 (2007) 1-22. https://doi.org/10.12989/was.2007.10.1.001.

[53] Y.Y. Lin, C.M. Cheng, C.H. Lee, A tuned mass damper for suppressing the coupled flexural and torsional buffeting response of long-span bridges, Eng. Struct. 22 (2000) 1195-1204. https://doi.org/10.1016/S0141-0296(99)00049-8.

[54] Y.L. Xu, K.C.S. Kwok, B. Samali, Control of wind-induced tall building vibration by tuned mass dampers, J. Wind Eng. Ind. Aerodyn. 40 (1992) 1-32. https://doi.org/10.1016/01676105(92)90518-F.

[55] K. Xu, K. Bi, Q. Han, X. Li, X. Du, Using tuned mass damper inerter to mitigate vortexinduced vibration of long-span bridges: Analytical study, Eng. Struct. 182 (2019) 101-111. https://doi.org/10.1016/j.engstruct.2018.12.067.

[56] Z. Jiang, The impact of a passive tuned mass damper on offshore single-blade installation, 
J. Wind Eng. Ind. Aerodyn. 176 (2018) 65-77. https://doi.org/10.1016/j.jweia.2018.03.008.

[57] F. Ubertini, G. Comanducci, S. Laflamme, A parametric study on reliability-based tunedmass damper design against bridge flutter, JVC/Journal Vib. Control. 23 (2017) 1518-1534. https://doi.org/10.1177/1077546315595304.

[58] K.C.S. Kwok, B. Samali, Performance of tuned mass dampers under wind loads, Eng. Struct. 17 (1995) 655-667. https://doi.org/10.1016/0141-0296(95)00035-6.

[59] R. Ma, X. Luo, Theory and application of multi-mode vibration control systems, Proc. Inst. Mech. Eng. Part C J. Mech. Eng. Sci. 227 (2013) 65-73. https://doi.org/10.1177/0954406212446114.

[60] G. Chen, J. Wu, Optimal Placement of Multiple Tune Mass Dampers for Seismic Structures, J. Struct. Eng. 127 (2001) 1054-1062. https://doi.org/10.1061/(asce)07339445(2001)127:9(1054).

[61] L.M. Sun, Y. Fujino, B.M. Pacheco, P. Chaiseri, Modelling of tuned liquid damper (TLD), J. Wind Eng. Ind. Aerodyn. 43 (1992) 1883-1894. https://doi.org/10.1016/01676105(92)90609-E.

[62] A. Casalotti, A. Arena, W. Lacarbonara, Mitigation of post-flutter oscillations in suspension bridges by hysteretic tuned mass dampers, Eng. Struct. 69 (2014) 62-71. https://doi.org/10.1016/j.engstruct.2014.03.001.

[63] Y. Lin, C. Cheng, Performance of multiple tuned mass dampers for suppressing buffeting response and increasing flutter speed of long-s-pan bridges, J. Chinese Inst. Eng. 24 (2001) 273-288. https://doi.org/10.1080/02533839.2001.9670626.

[64] E. Limer, How a Skyscraper Stays Upright in a Typhoon, (n.d.). https://www.popularmechanics.com/technology/design/a16819/tapei-101-mass-damper- 
record/ (accessed July 6, 2021).

[65] P. Soltani, A. Deraemaeker, Pendulum tuned mass dampers and tuned mass dampers: Analogy and optimum parameters for various combinations of response and excitation $\begin{array}{llll}\text { parameters, } & \text { JVC/Journal } & \text { Vib. } & \text { Control. }\end{array}$ https://doi.org/10.1177/10775463211003414.

[66] A.J. Roffel, S. Narasimhan, Results from a Full-Scale Study on the Condition Assessment of Pendulum Tuned Mass Dampers, J. Struct. Eng. 142 (2016) 04015096. https://doi.org/10.1061/(asce)st.1943-541x.0001339.

[67] B.P.D. Tuong, P.D. Huynh, T.-T. Bui, V. Sarhosis, Numerical Analysis of the Dynamic Responses of Multistory Structures Equipped with Tuned Liquid Dampers Considering Fluid-Structure Interactions, Open Constr. Build. Technol. J. 13 (2019) 289-300. https://doi.org/10.2174/1874836801913010289.

[68] A.F. de S. Neto, Optimization of Discrete Dynamic Parameters in TLD Coupled to Structures, University of Brasilia, 2018. https://bdm.unb.br/handle/10483/25203.

[69] Z. Zhang, A. Staino, B. Basu, S.R.K. Nielsen, Performance evaluation of full-scale tuned liquid dampers (TLDs) for vibration control of large wind turbines using real-time hybrid testing, Eng. Struct. 126 (2016) 417-431. https://doi.org/10.1016/j.engstruct.2016.07.008.

[70] H. Kim, H. Adeli, Wind-Induced Motion Control of 76-Story Benchmark Building Using the Hybrid Damper-TLCD System, J. Struct. Eng. 131 (2005) 1794-1802. https://doi.org/10.1061/(asce)0733-9445(2005)131:12(1794).

[71] J.S. Love, M.J. Tait, The influence of tank orientation angle on a 2D structure-tuned liquid damper system, J. Vib. Acoust. Trans. ASME. 135 (2013) 1-11. https://doi.org/10.1115/1.4007416. 
[72] I. Nishimura, T. Kobori, M. Sakamoto, N. Koshika, K. Sasaki, S. Ohrui, Active tuned mass damper, Smart Mater. Struct. 1 (1992) 306-311. https://doi.org/10.1088/0964$1726 / 1 / 4 / 005$.

[73] C.C. Chang, H.T.Y. Yang, Control of Buildings Using Active Tuned Mass Dampers, J. Eng. Mech. 121 (1995) 355-366. https://doi.org/10.1061/(ASCE)0733-9399(1995)121:3(355).

[74] B.F. Spencer, M.K. Sain, Controlling buildings: a new frontier in feedback, IEEE Control Syst. Mag. 17 (1997) 19-35. https://doi.org/10.1109/37.642972.

[75] T. Engle, H. Mahmoud, A. Chulahwat, Hybrid Tuned Mass Damper and Isolation Floor Slab System Optimized for Vibration Control, J. Earthq. Eng. 19 (2015) 1197-1221. https://doi.org/10.1080/13632469.2015.1037406.

[76] C.-C. Lin, G.-L. Lin, J.-F. Wang, Protection of seismic structures using semi-active friction TMD, Earthq. $\quad$ Eng. $\quad \backslash \& \quad$ Struct. $\quad$ Dyn. $39 \quad(2010) \quad 635-659$. https://doi.org/https://doi.org/10.1002/eqe.961.

[77] S. Nagarajaiah, E. Sonmez, Structures with Semiactive Variable Stiffness Single/Multiple

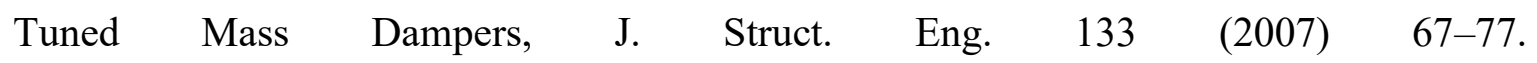
https://doi.org/10.1061/(asce)0733-9445(2007)133:1(67).

[78] G.M. Chatziathanasiou, N.A. Chrysochoidis, D.A. Saravanos, A semi-active shunted piezoelectric tuned mass damper for robust vibration control, J. Vib. Control. (2021) 107754632110264. https://doi.org/10.1177/10775463211026487.

[79] F. Weber, Semi-active vibration absorber based on real-time controlled MR damper, Mech. Syst. Signal Process. 46 (2014) 272-288. https://doi.org/10.1016/j.ymssp.2014.01.017.

[80] N. Hoang, Y. Fujino, P. Warnitchai, Optimal tuned mass damper for seismic applications and practical design formulas, Eng. Struct. $30 \quad$ (2008) $707-715$. 
https://doi.org/10.1016/j.engstruct.2007.05.007.

[81] O. Lavan, Multi-objective optimal design of tuned mass dampers, Struct. Control Heal. Monit. 24 (2017) 1-16. https://doi.org/10.1002/stc.2008.

[82] G. Bekdaş, S.M. Nigdeli, X.S. Yang, A novel bat algorithm based optimum tuning of mass dampers for improving the seismic safety of structures, Eng. Struct. 159 (2018) 89-98. https://doi.org/10.1016/j.engstruct.2017.12.037.

[83] X.-S. Yang, Chapter 10 - Bat Algorithms, in: X.-S. Yang (Ed.), Nature-Inspired Optim. Algorithms, Elsevier, Oxford, 2014: pp. 141-154. https://doi.org/https://doi.org/10.1016/B978-0-12-416743-8.00010-5.

[84] S.M. Nigdeli, G. Bekdaş, Optimum design of multiple positioned tuned mass dampers for structures constrained with axial force capacity, Struct. Des. Tall Spec. Build. 28 (2019) 116. https://doi.org/10.1002/tal.1593.

[85] Y. Liu, K. Wang, O. Mercan, H. Chen, P. Tan, Experimental and numerical studies on the optimal design of tuned mass dampers for vibration control of high-rise structures, Eng. Struct. 211 (2020) 110486. https://doi.org/10.1016/j.engstruct.2020.110486.

[86] A. Batou, S. Adhikari, Optimal parameters of viscoelastic tuned-mass dampers, J. Sound Vib. 445 (2019) 17-28. https://doi.org/10.1016/j.jsv.2019.01.010.

[87] M. Khatibinia, H. Gholami, R. Kamgar, Optimal design of tuned mass dampers subjected to continuous stationary critical excitation, Int. J. Dyn. Control. 6 (2018) 1094-1104. https://doi.org/10.1007/s40435-017-0386-7.

[88] M. Mohebbi, K. Shakeri, Y. Ghanbarpour, H. Majzoub, Designing optimal multiple tuned mass dampers using genetic algorithms (GAs) for mitigating the seismic response of $\begin{array}{llllll}\text { structures, JVC/Journal } & \text { Vib. } & \text { Control. } & 19 & \text { (2013) }\end{array}$ 
https://doi.org/10.1177/1077546311434520.

[89] M. Petyt, Introduction to Finite Element Vibration Analysis, Cambridge University Press, Cambridge, UK, 1998. https://books.google.ca/books?id=QUTHYCAtRhcC.

[90] L. Meirovitch, Analytical Methods in Vibrations, Macmillan, 1967. https://books.google.ca/books?id=sf1QAAAAMAAJ.

[91] W.P. Rodden, Aerodynamic Influence Coefficients from Strip Theory, J. Aerosp. Sci. 26 (1959) 833-834. https://doi.org/10.2514/8.8333.

[92] P. Konstadinopoulos, D.F. Thrasher, D.T. Mook, A.H. Nayfeh, L. Watson, A vortex-lattice method for general, unsteady aerodynamics, J. Aircr. 22 (1985) 43-49. https://doi.org/10.2514/3.45078.

[93] E. Albano, W.P. Rodden, A doublet-lattice method for calculating lift distributions on oscillating surfaces in subsonic flows, AIAA J. 7 (1969) 279-285. https://doi.org/10.2514/3.5086.

[94] K. Roughen, M. Baker, T. Fogarty, Computational Fluid Dynamics and Doublet-Lattice Calculation of Unsteady Control Surface Aerodynamics, J. Guid. Control Dyn. - J Guid Control DYNAM. 24 (2001) 160-166. https://doi.org/10.2514/2.4697.

[95] C. Valente, Y. Lemmens, C. Wales, D. Jones, A.L. Gaitonde, J.E. Cooper, A doublet-lattice method correction approach for high fidelity gust loads analysis, 58th AIAA/ASCE/AHS/ASC Struct. Struct. Dyn. Mater. Conf. 2017. (2017) 1-17. https://doi.org/10.2514/6.2017-0632.

[96] R.H. MacNeal, The NASTRAN Theoretical Manual, Scientific and Technical Information Office, National Aeronautics and Space Administration, 1970. https://books.google.ca/books?id=p51YAAAAYAAJ. 
[97] J.R. Fuller, Evolution of airplane gust loads design requirements, J. Aircr. 32 (1995) 235246. https://doi.org/10.2514/3.46709.

[98] J.R. Wright, J.E. Cooper, Introduction to Aircraft Aeroelasticity and Loads, John Wiley, 2007. https://books.google.ca/books?id=y7a7kQEACAAJ.

[99] A.G. Davenport, The spectrum of horizontal gustiness near the ground in high winds, Q. J. R. Meteorol. Soc. 88 (1962) 197-198. https://doi.org/10.1002/qj.49708837618.

[100] A.G. Davenport, The Dependence of Wind Load on Meteorological Parameters, 1971., 1971. https://books.google.ca/books?id=vBvLtAEACAAJ.

[101] W. Gawronski, Modeling wind-gust disturbances for the analysis of antenna pointing accuracy, IEEE Antennas Propag. Mag. $46 \quad$ (2004) 50-58. https://doi.org/10.1109/MAP.2004.1296144.

[102] W. Gawronski, B. Bienkiewicz, R. Hill, Wind-induced Dynamics Of A Deep Space Network Antenna, J. Sound Vib. - J SOUND VIB. 178 (1994) 67-77. https://doi.org/10.1006/jsvi.1994.1468.

[103] W.K. Gawronski, J.A. Mellstrom, Control and dynamics of the deep space network antennas, Control Dyn. Syst. 63 (1994) 289.

[104] C. Van Loan, Computational frameworks for the fast Fourier transform, SIAM, 1992.

[105] J.H. Holland, J.H. Holland, P.P.E.E.C.S.J.H. Holland, Adaptation in Natural and Artificial Systems: An Introductory Analysis with Applications to Biology, Control, and Artificial Intelligence, University of $\quad 1975$. https://books.google.ca/books?id=JE5RAAAAMAAJ.

[106] A.F. Izmailov, M. V Solodov, Newton-Type Methods for Optimization and Variational

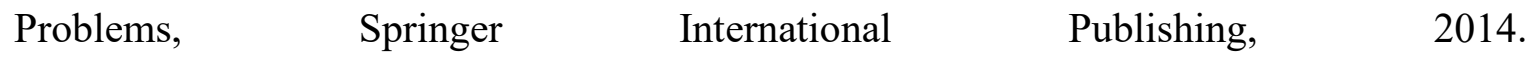


https://books.google.ca/books?id=uMy6BQAAQBAJ.

[107] B. Mendelson, Introduction to Topology, Dover Publications, 2012. https://books.google.ca/books?id=FWFmoEUJSwkC.

[108] M. Mitchell, An Introduction to Genetic Algorithms, Bradford Books, 1998. https://books.google.ca/books?id=0eznlz0TF-IC.

[109] D.E. Goldberg, G. David Edward, D.E.G. Goldberg, V.A.P.H.D.E. Goldberg, Genetic Algorithms in Search, Optimization, and Machine Learning, Addison-Wesley Publishing Company, 1989. https://books.google.ca/books?id=2IIJAAAACAAJ.

[110] ESTECO, modeFRONTIER software, (n.d.). https://engineering.esteco.com/modefrontier/ (accessed July 20, 2021).

[111] MathWorks, MATLAB software, $\quad$ (n.d.). https://www.mathworks.com/products/matlab.html (accessed July 20, 2021).

[112] Palisade, Evolver software, (n.d.). https://www.palisade.com/evolver/default.asp (accessed July 20, 2021).

[113] Y. Deng, Y. Liu, D. Zhou, An Improved Genetic Algorithm with Initial Population Strategy for Symmetric TSP, Math. Probl. Eng. 2015 (2015). https://doi.org/10.1155/2015/212794.

[114] J.E. Baker, Adaptive Selection Methods for Genetic Algorithms, in: Proc. 1st Int. Conf. Genet. Algorithms, L. Erlbaum Associates Inc., USA, 1985: pp. 101-111.

[115] Z. Michalewicz, M. Schmidt, Evolutionary Algorithms and Constrained Optimization, in: Evol. Optim., Kluwer Academic Publishers, Boston, n.d. https://doi.org/10.1007/0-30648041-7_3.

[116] K. Deep, M. Thakur, A new crossover operator for real coded genetic algorithms, Appl. Math. Comput. 188 (2007) 895-911. https://doi.org/10.1016/j.amc.2006.10.047. 
$\begin{array}{llll}\text { [117] MathWorks, } & \text { Genetic } & \text { Optgorithms, }\end{array}$ https://www.mathworks.com/help/gads/genetic-algorithm-options.html\#f6633 (accessed May 5, 2021).

[118] S. Marsili Libelli, P. Alba, Adaptive mutation in genetic algorithms, Soft Comput. 4 (2000) 76-80. https://doi.org/10.1007/s005000000042.

[119] Y. Censor, Pareto optimality in multiobjective problems, Appl. Math. Optim. 4 (1977) 4159. https://doi.org/10.1007/BF01442131.

[120] N.. Da Cunha, E. Polak, Constrained minimization under vector-valued criteria in finite dimensional spaces, J. Math. Anal. Appl. 19 (1967) 103-124. https://doi.org/10.1016/0022247X(67)90025-X.

[121] L. Zadeh, Optimality and non-scalar-valued performance criteria, IEEE Trans. Automat. Contr. 8 (1963) 59-60. https://doi.org/10.1109/TAC.1963.1105511.

[122] K. Kilroy, MSC Nastran - Quick Reference Guide, MacNeal-Schwendler Corporation, 1998. https://books.google.ca/books?id=sLR_wgEACAAJ.

[123] L. Lu, C.M. Anderson-Cook, T.J. Robinson, Optimization of designed experiments based on multiple criteria utilizing a pareto frontier, Technometrics. 53 (2011) 353-365. https://doi.org/10.1198/TECH.2011.10087.

[124] A. Szparaga, M. Stachnik, E. Czerwińska, S. Kocira, M. Dymkowska-Malesa, M. Jakubowski, Multi-objective optimization based on the utopian point method applied to a case study of osmotic dehydration of plums and its storage, J. Food Eng. 245 (2019) 104111. https://doi.org/10.1016/j.jfoodeng.2018.10.014.

[125] M. Inoue, J.C. Algaba-Marcos, K. Asada, R. Blundell, W. Brisken, R. Burgos, C.-. Chang, M.-. Chen, S.S. Doeleman, V. Fish, P. Grimes, J. Han, H. Hirashita, P.T.P. Ho, S.-. Hsieh, 
T. Huang, H. Jiang, E. Keto, P.M. Koch, D.Y. Kubo, C.-. Kuo, B. Liu, P. Martin-Cocher, S. Matsushita, Z. Meyer-Zhao, M. Nakamura, P. Napier, H. Nishioka, G. Nystrom, S. Paine, N. Patel, N. Pradel, H.-. Pu, P.A. Raffin, H.-. Shen, W. Snow, R. Srinivasan, T.-. Wei, Greenland telescope project: Direct confirmation of black hole with sub-millimeter VLBI, Radio Sci. 49 (2014) 564-571. https://doi.org/10.1002/2014RS005450.

[126] T. Hobiger, T. Kondo, H. Schuh, Very long baseline interferometry as a tool to probe the ionosphere, Radio Sci. 41 (2006) 1-10. https://doi.org/10.1029/2005RS003297.

[127] A. Brunthaler, M.J. Reid, K.M. Menten, X.-W. Zheng, A. Bartkiewicz, Y.K. Choi, T. Dame, K. Hachisuka, K. Immer, G. Moellenbrock, L. Moscadelli, K.L.J. Rygl, A. Sanna, M. Sato, Y. Wu, Y. Xu, B. Zhang, The Bar and Spiral Structure Legacy (BeSSeL) survey: Mapping the Milky Way with VLBI astrometry, Astron. Nachrichten. 332 (2011) 461-466. https://doi.org/https://doi.org/10.1002/asna.201111560.

[128] W.D. Cotton, Very Long Baseline Interferometry, in: High Angular Resolut. Astrophys., Springer Netherlands, Dordrecht, 1997: pp. 165-191. https://doi.org/10.1007/978-94-0090041-7_9.

[129] Matweb, Aluminum 6061-T6, (2021). http://www.matweb.com/search/datasheet.aspx?MatGUID=1b8c06d0ca7c456694c7777d9 e10be5b (accessed March 26, 2021).

[130] Siemens, Superelement User's $\quad$ Guide, $\quad$ (2016) 240. https://docs.plm.automation.siemens.com/data_services/resources/nxnastran/10/help/en_U S/tdocExt/pdf/super.pdf.

[131] R.R. CRAIG, M.C.C. BAMPTON, Coupling of substructures for dynamic analyses, AIAA J. 6 (1968) 1313-1319. https://doi.org/10.2514/3.4741. 
[132] P.V. Thomas, M.S.A. ElSayed, D. Walch, Review of Model Order Reduction Methods and Their Applications in Aeroelasticity Loads Analysis for Design Optimization of Complex Airframes, J. Aerosp. Eng. 32 (2019) 04018156. https://doi.org/10.1061/(asce)as.19435525.0000972.

[133] R.J. Allemang, The modal assurance criterion - Twenty years of use and abuse, Sound Vib. 37 (2003) 14-21.

[134] G.B. Warburton, Optimum absorber parameters for various combinations of response and excitation parameters, Earthq. Eng. Struct. Dyn. $10 \quad$ (1982) 381-401. https://doi.org/10.1002/eqe.4290100304. 